Supporting Information

\title{
Regioselective and Diastereoselective Dearomative
}

\section{Multifunctionalization of In-Situ-Activated Azaarenes: An Access to Bridged Azaheterocycles}

\author{
Xu-Guan Bai, ${ }^{a}$ Hong-Jie Miao, ${ }^{\mathrm{a}}$ Yang Zhao, ${ }^{\mathrm{a}}$ Qi-Lin Wang ${ }^{\mathrm{a}}{ }^{\mathrm{a}}$ and Zhan-Wei Bu ${ }^{\mathrm{a}}$ \\ ${ }^{a}$ Institute of Functional Organic Molecular Engineering, College of Chemistry and Chemical \\ Engineering, Henan University, Kaifeng 475004, China \\ E-mail: wangqilin@henu.edu.cn; buzhanwei@henu.edu.cn
}

Table of Contents

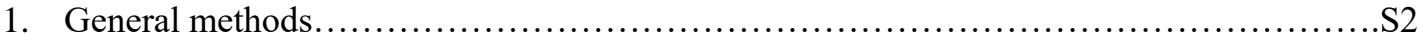

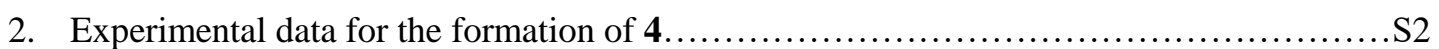

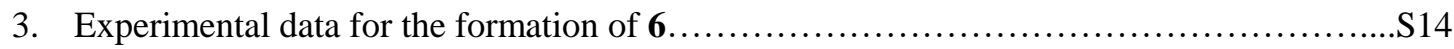

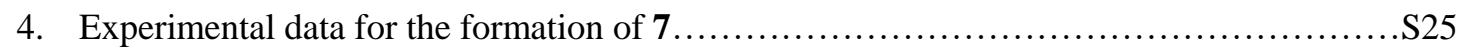

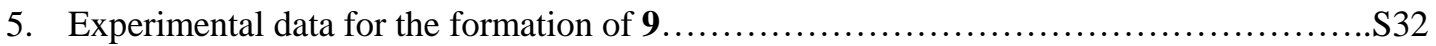

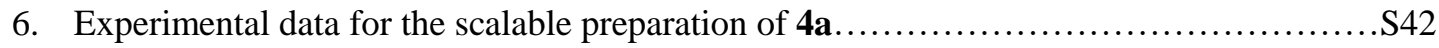

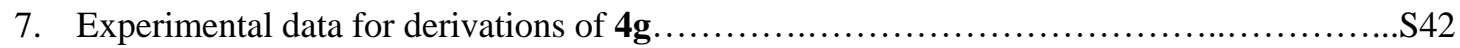

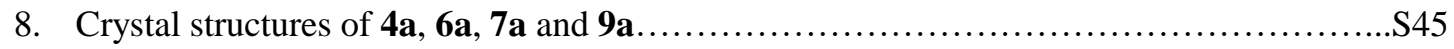

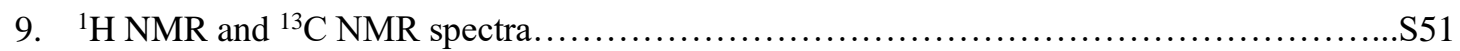




\section{General methods}

NMR spectra were recorded with tetramethylsilane as the internal standard. ${ }^{1} \mathrm{H}$ NMR spectra were recorded at $400 \mathrm{MHz}$ and some at $300 \mathrm{MHz}$, and ${ }^{13} \mathrm{C}$ NMR spectra were recorded at $100 \mathrm{MHz}$ and some at $75 \mathrm{MHz}$ (Bruker Avance). ${ }^{1} \mathrm{H}$ NMR chemical shifts $(\delta)$ are reported in ppm relative to tetramethylsilane (TMS) with the solvent signal as the internal standard $\left(\mathrm{CDCl}_{3}\right.$ at $7.26 \mathrm{ppm}$, $\left(\mathrm{CD}_{3}\right)_{2} \mathrm{SO}$ at $\left.2.50 \mathrm{ppm}\right) .{ }^{13} \mathrm{C}$ NMR chemical shifts are reported in ppm from tetramethylsilane (TMS) with the solvent resonance as the internal standard $\left(\mathrm{CDCl}_{3}\right.$ at $77.00 \mathrm{ppm},\left(\mathrm{CD}_{3}\right)_{2} \mathrm{SO}$ at $39.52 \mathrm{ppm}$ ). Data are given as: s (singlet), d (doublet), $\mathrm{t}$ (triplet), q (quartet), dd (double of doublet), br (broad) or m (multiplets), coupling constants (Hz) and integration. Flash column chromatography was carried out using silica gel eluting with ethyl acetate and petroleum ether. High resolution mass spectra were obtained with the Q-TOF-Premier mass spectrometer. Reactions were monitored by TLC and visualized with ultraviolet light. IR spectra were recorded on a Thermo Fisher Nicolet Avatar 360 FTIR spectrometer on a KBr beam splitter. All the solvents were used directly without any purification.

\section{Experimental data for the formation of 4}
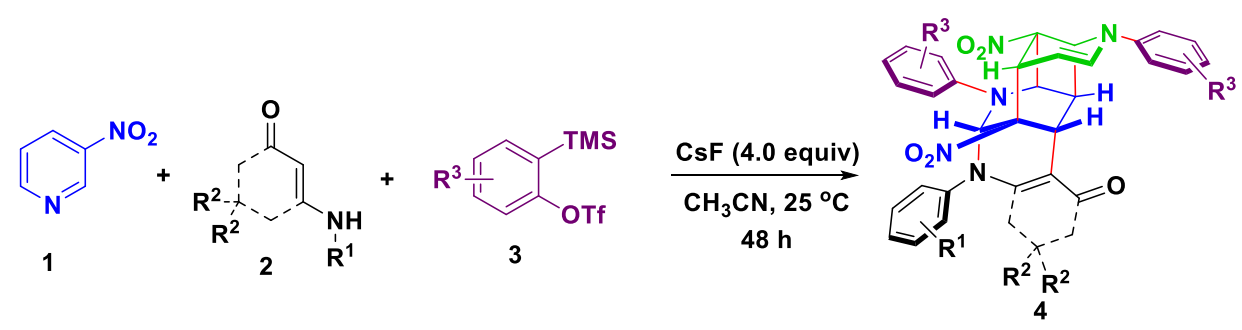

General procedure: To a $5.0 \mathrm{~mL}$ vial were successively added 3-nitropyridine $\mathbf{1}(0.30 \mathrm{mmol})$, enaminones $2(0.15 \mathrm{mmol})$, aryne precursors $3(0.60 \mathrm{mmol}), \mathrm{CsF}(0.60 \mathrm{mmol})$ and $1.0 \mathrm{~mL}$ of $\mathrm{CH}_{3} \mathrm{CN}$. The resulting mixture was stirred at $25{ }^{\circ} \mathrm{C}$ for $48 \mathrm{~h}$, and then the reaction mixture was directly subjected to flash column chromatography on silica gel (petroleum ether/ ethyl acetate) to afford the corresponding products $\mathbf{4}$. For some cases, such as $\mathbf{4 a - c}$ and $\mathbf{4 e - h}$, the products precipitated out from the reaction systems and only a filtration was needed to purify them.

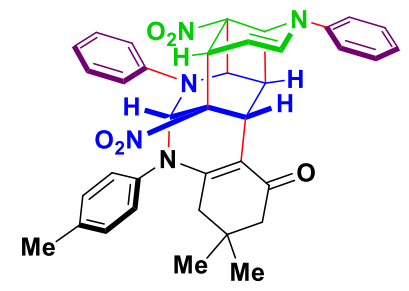


3,3-dimethyl-7b,13-dinitro-7,11-diphenyl-5-( $p$-tolyl)-3,4,5,6,7,7a,7b, 8,11,11a,11b,12-dodecahydro -6,8,12-(epimethanetriyl)benzo[d]pyrido[3',2':3,4]cyclobuta[1,2-g][1,3]diazocin-1(2H)-one $(\mathbf{4 a})$ Yellow solid obtained by filtration of the precipitate; $80.3 \mathrm{mg}, 85 \%$ yield; $\mathrm{dr}>20: 1$; reaction time $=48 \mathrm{~h} ; \mathrm{mp} 218.2-218.9^{\circ} \mathrm{C} ;{ }^{1} \mathrm{H}$ NMR $\left(400 \mathrm{MHz}, \mathrm{CDCl}_{3}\right) \delta 7.31(\mathrm{t}, J=8.0 \mathrm{~Hz}, 2 \mathrm{H}), 7.14(\mathrm{t}, J=8.0$ $\mathrm{Hz}, 4 \mathrm{H}), 7.00(\mathrm{q}, J=8.0 \mathrm{~Hz}, 2 \mathrm{H}), 6.88(\mathrm{t}, J=8.0 \mathrm{~Hz}, 5 \mathrm{H}), 6.37(\mathrm{br}, 2 \mathrm{H}), 5.96(\mathrm{~s}, 1 \mathrm{H}), 4.45\left(\mathrm{dd}, J_{I}\right.$ $\left.=J_{2}=4.0 \mathrm{~Hz}, 1 \mathrm{H}\right), 4.27(\mathrm{t}, J=8.0 \mathrm{~Hz}, 1 \mathrm{H}), 4.13(\mathrm{~d}, J=8.0 \mathrm{~Hz}, 1 \mathrm{H}), 3.99\left(\mathrm{dd}, J_{l}=J_{2}=4.0 \mathrm{~Hz}\right.$, 1H), $3.95(\mathrm{~s}, 1 \mathrm{H}), 3.19(\mathrm{t}, J=8.0 \mathrm{~Hz}, 1 \mathrm{H}), 2.23-2.13(\mathrm{~m}, 5 \mathrm{H}), 1.87(\mathrm{t}, J=16.0 \mathrm{~Hz}, 2 \mathrm{H}), 0.87(\mathrm{~s}$, 3H), $0.84(\mathrm{~s}, 3 \mathrm{H}) ;{ }^{13} \mathrm{C}$ NMR (100 MHz, $\left.\mathrm{CDCl}_{3}\right) \delta$ 194.2, 153.6, 145.5, 145.1, 139.6, 137.8, 134.0, 130.0, 129.9, 129.6, 129.6, 124.7, 123.6, 123.5, 118.7, 107.4, 87.4, 85.6, 84.4, 59.7, 52.5, 50.0, 48.5, 41.5, 38.5, 32.8, 29.1, 26.7, 25.3, 20.9. IR (KBr) v 3431, 2957, 1587, 1542, 1266, $756 \mathrm{~cm}^{-1}$. HRMS (ESI) calcd for $\mathrm{C}_{37} \mathrm{H}_{36} \mathrm{~N}_{5} \mathrm{O}_{5}[\mathrm{M}+\mathrm{H}]^{+}$630.2711, found 630.2702.

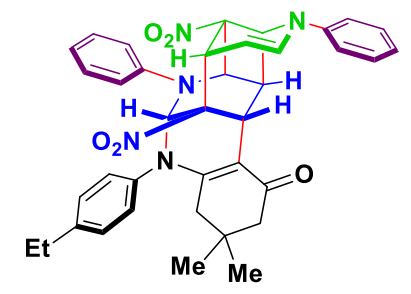

5-(4-ethylphenyl)-3,3-dimethyl-7b,13-dinitro-7,11-diphenyl-3,4,5,6,7,7a,7b,8,11,11a,11b,12-dode cahydro-6,8,12-(epimethanetriyl)benzo[ $d]$ pyrido[3',2':3,4]cyclobuta[1,2-g][1,3]diazocin-1(2H)-on e $(\mathbf{4 b})$

Yellow solid obtained by filtration of the precipitate; $72.1 \mathrm{mg}, 75 \%$ yield; $\mathrm{dr}>20: 1$; reaction time $=48 \mathrm{~h} ; \mathrm{mp} 219.9-220.6{ }^{\circ} \mathrm{C} ;{ }^{1} \mathrm{H}$ NMR $\left(400 \mathrm{MHz}, \mathrm{CDCl}_{3}\right) \delta 7.32(\mathrm{t}, J=8.0 \mathrm{~Hz}, 2 \mathrm{H}), 7.15(\mathrm{t}, J=8.0$ $\mathrm{Hz}, 4 \mathrm{H}), 7.05-6.86(\mathrm{~m}, 7 \mathrm{H}), 6.40(\mathrm{br}, 2 \mathrm{H}), 5.97(\mathrm{~s}, 1 \mathrm{H}), 4.46\left(\mathrm{dd}, J_{l}=J_{2}=4.0 \mathrm{~Hz}, 1 \mathrm{H}\right), 4.29(\mathrm{t}, J=$ $8.0 \mathrm{~Hz}, 1 \mathrm{H}), 4.14(\mathrm{~d}, J=8.0 \mathrm{~Hz}, 1 \mathrm{H}), 3.99\left(\mathrm{dd}, J_{l}=J_{2}=4.0 \mathrm{~Hz}, 1 \mathrm{H}\right), 3.96(\mathrm{~s}, 1 \mathrm{H}), 3.19(\mathrm{t}, J=8.0$ $\mathrm{Hz}, 1 \mathrm{H}), 2.52(\mathrm{q}, J=16.0 \mathrm{~Hz}, 2 \mathrm{H}), 2.24-2.14(\mathrm{~m}, 2 \mathrm{H}), 1.90(\mathrm{~s}, 2 \mathrm{H}), 1.13(\mathrm{t}, J=8.0 \mathrm{~Hz}, 3 \mathrm{H}), 0.89$ (s, 3H), $0.86(\mathrm{~s}, 3 \mathrm{H}) ;{ }^{13} \mathrm{C} \mathrm{NMR}\left(100 \mathrm{MHz}, \mathrm{CDCl}_{3}\right) \delta 194.3,153.7,145.6,145.2,144.1,139.8$, 134.1, 129.7, 129.6, 128.7, 124.8, 124.2, 123.6, 123.5, 118.8, 107.5, 87.5, 85.7, 84.5, 59.8, 52.6, $50.1,48.6,41.6,38.6,32.9,29.1,28.3,26.8,25.4,15.3$, one carbon missing in the aromatic region. IR $(\mathrm{KBr}) v 3439,2960,1633,1582,1541,1501,1358,1294,751 \mathrm{~cm}^{-1}$. HRMS (ESI) calcd for $\mathrm{C}_{38} \mathrm{H}_{38} \mathrm{~N}_{5} \mathrm{O}_{5}[\mathrm{M}+\mathrm{H}]^{+}$644.2867, found 644.2870. 


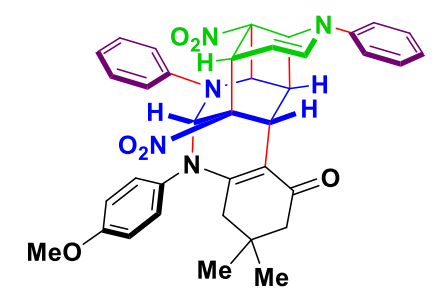

5-(4-methoxyphenyl)-3,3-dimethyl-7b,13-dinitro-7,11-diphenyl-3,4,5,6,7,7a,7b,8,11,11a,11b,12-d odecahydro-6,8,12-(epimethanetriyl)benzo[d]pyrido[3',2':3,4]cyclobuta[1,2-g][1,3]diazocin-1(2H) -one $(4 \mathrm{c})$

Yellow solid obtained by filtration of the precipitate; $70.1 \mathrm{mg}, 72 \%$ yield; $\mathrm{dr}>20: 1$; reaction time $=48 \mathrm{~h} ; \mathrm{mp} 233.1-233.7^{\circ} \mathrm{C} ;{ }^{1} \mathrm{H}$ NMR $\left(400 \mathrm{MHz}, \mathrm{CDCl}_{3}\right) \delta 7.39(\mathrm{t}, J=8.0 \mathrm{~Hz}, 2 \mathrm{H}), 7.22(\mathrm{t}, J=8.0$ $\mathrm{Hz}, 4 \mathrm{H}), 7.12-7.04(\mathrm{~m}, 2 \mathrm{H}), 6.98-6.93(\mathrm{~m}, 3 \mathrm{H}), 6.67(\mathrm{br}, 4 \mathrm{H}), 6.05(\mathrm{~s}, 1 \mathrm{H}), 4.52\left(\mathrm{dd}, J_{l}=J_{2}=4.0\right.$ $\mathrm{Hz}, 1 \mathrm{H}), 4.35(\mathrm{t}, J=8.0 \mathrm{~Hz}, 1 \mathrm{H}), 4.22(\mathrm{~d}, J=8.0 \mathrm{~Hz}, 1 \mathrm{H}), 4.06\left(\mathrm{dd}, J_{l}=J_{2}=4.0 \mathrm{~Hz}, 1 \mathrm{H}\right), 4.04(\mathrm{~s}$, 1H), $3.75(\mathrm{~s}, 3 \mathrm{H}), 3.26(\mathrm{t}, J=8.0 \mathrm{~Hz}, 1 \mathrm{H}), 2.30-2.20(\mathrm{~m}, 2 \mathrm{H}), 1.93(\mathrm{q}, J=16.0 \mathrm{~Hz}, 2 \mathrm{H}), 0.95(\mathrm{~s}$, 3H), $0.91(\mathrm{~s}, 3 \mathrm{H}) ;{ }^{13} \mathrm{C}$ NMR (100 MHz, $\left.\mathrm{CDCl}_{3}\right) \delta$ 194.1, 158.8, 153.8, 145.5, 145.2, 135.0, 134.0, 129.6, 129.6, 124.6, 123.6, 123.2, 118.8, 114.3, 107.0, 87.5, 85.6, 84.4, 59.6, 55.4, 52.6, 50.0, 48.6, $41.5,38.5,32.7,29.2,26.7,25.3$, one carbon missing in the aromatic region. $\mathrm{IR}(\mathrm{KBr}) v 3430$, 2955, 1579, 1502, 1297, 1235, $745 \mathrm{~cm}^{-1}$. HRMS (ESI) calcd for $\mathrm{C}_{37} \mathrm{H}_{36} \mathrm{~N}_{5} \mathrm{O}_{6}[\mathrm{M}+\mathrm{H}]^{+} 646.2660$, found 646.2647 .

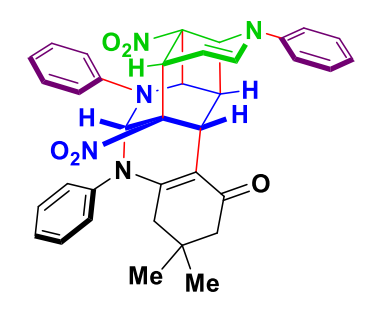

3,3-dimethyl-7b,13-dinitro-5,7,11-triphenyl-3,4,5,6,7,7a,7b,8,11,11a,11b,12-dodecahydro-6,8,12-( epimethanetriyl)benzo[d]pyrido[3',2':3,4]cyclobuta[1,2-g][1,3]diazocin-1(2H)-one (4d) Yellow solid obtained by column chromatography (petroleum ether/ethyl acetate $=10: 1$ to $5: 1$ ); $76.2 \mathrm{mg}, 83 \%$ yield; $\mathrm{dr}>20: 1$; reaction time $=48 \mathrm{~h}$; mp 178.2-178.9 ${ }^{\circ} \mathrm{C} ;{ }^{1} \mathrm{H}$ NMR $(400 \mathrm{MHz}$, $\left.\mathrm{CDCl}_{3}\right) \delta 7.40(\mathrm{t}, J=8.0 \mathrm{~Hz}, 2 \mathrm{H}), 7.26-7.20(\mathrm{~m}, 6 \mathrm{H}), 7.13-7.06(\mathrm{~m}, 2 \mathrm{H}), 6.96\left(\mathrm{dd}, J_{l}=J_{2}=8.0 \mathrm{~Hz}\right.$, $3 \mathrm{H}), 6.59(\mathrm{br}, 2 \mathrm{H}), 6.07(\mathrm{~s}, 1 \mathrm{H}), 4.54(\mathrm{~d}, J=4.0 \mathrm{~Hz}, 1 \mathrm{H}), 4.36(\mathrm{t}, J=8.0 \mathrm{~Hz}, 1 \mathrm{H}), 4.21(\mathrm{~d}, J=8.0$ $\mathrm{Hz}, 1 \mathrm{H}), 4.07(\mathrm{~d}, J=4.0 \mathrm{~Hz}, 1 \mathrm{H}), 4.05(\mathrm{~s}, 1 \mathrm{H}), 3.27(\mathrm{t}, J=8.0 \mathrm{~Hz}, 1 \mathrm{H}), 2.27(\mathrm{t}, J=16.0 \mathrm{~Hz}, 2 \mathrm{H})$, 2.00-1.92 (m, 3H), 0.97 (s, 3H), 0.93 (s, 3H); $\left.{ }^{13} \mathrm{C} \mathrm{NMR} \mathrm{(100} \mathrm{MHz,} \mathrm{CDCl}_{3}\right) \delta 194.3,153.3,145.5$, $145.2,142.3,134.1,129.7,129.6,129.4,129.4,127.8,124.9,123.7,123.6,118.8,107.7,87.5$, 
85.6, 84.5, 59.8, 52.6, 50.1, 48.6, 41.7, 38.6, 33.0, 29.2, 26.8, 25.4. IR (KBr) v 3441, 2954, 1588, 1544, $756 \mathrm{~cm}^{-1}$. HRMS (ESI) calcd for $\mathrm{C}_{36} \mathrm{H}_{34} \mathrm{~N}_{5} \mathrm{O}_{5}[\mathrm{M}+\mathrm{H}]^{+}$616.2554, found 616.2540.

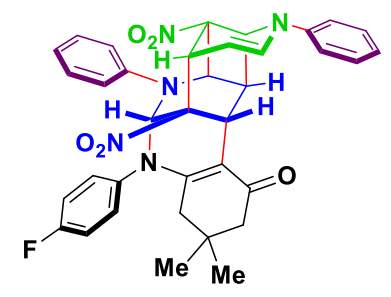

5-(4-fluorophenyl)-3,3-dimethyl-7b,13-dinitro-7,11-diphenyl-3,4,5,6,7,7a,7b,8,11,11a,11b,12-dod ecahydro-6,8,12-(epimethanetriyl)benzo[ $d]$ pyrido[3',2':3,4]cyclobuta[1,2-g][1,3] diazocin-1(2H)-o ne $(\mathbf{4 e})$

Yellow solid obtained by filtration of the precipitate; $69.7 \mathrm{mg}, 73 \%$ yield; $\mathrm{dr}>20: 1$; reaction time $=48 \mathrm{~h} ; \mathrm{mp} 143.7-144.5^{\circ} \mathrm{C} ;{ }^{1} \mathrm{H}$ NMR $\left(400 \mathrm{MHz}, \mathrm{CDCl}_{3}\right) \delta 7.40(\mathrm{t}, J=8.0 \mathrm{~Hz}, 2 \mathrm{H}), 7.22(\mathrm{t}, J=8.0$ $\mathrm{Hz}, 4 \mathrm{H}), 7.11(\mathrm{t}, J=8.0 \mathrm{~Hz}, 2 \mathrm{H}), 6.97(\mathrm{t}, J=8.0 \mathrm{~Hz}, 5 \mathrm{H}), 6.57(\mathrm{br}, 2 \mathrm{H}), 6.05(\mathrm{~s}, 1 \mathrm{H}), 4.53(\mathrm{~d}, J=$ $8.0 \mathrm{~Hz}, 1 \mathrm{H}), 4.36(\mathrm{t}, J=8.0 \mathrm{~Hz}, 1 \mathrm{H}), 4.19(\mathrm{~d}, J=8.0 \mathrm{~Hz}, 1 \mathrm{H}), 4.07(\mathrm{~d}, J=8.0 \mathrm{~Hz}, 1 \mathrm{H}), 4.04(\mathrm{~s}$, 1H), $3.26(\mathrm{t}, J=8.0 \mathrm{~Hz}, 1 \mathrm{H}), 2.27(\mathrm{t}, J=16.0 \mathrm{~Hz}, 2 \mathrm{H}), 2.00-1.85(\mathrm{~m}, 2 \mathrm{H}), 0.97(\mathrm{~s}, 3 \mathrm{H}), 0.92(\mathrm{~s}$, $3 \mathrm{H}) ;{ }^{13} \mathrm{C}$ NMR $\left(100 \mathrm{MHz}, \mathrm{CDCl}_{3}\right) \delta 194.3,161.6(\mathrm{~d}, J=247.0 \mathrm{~Hz}, 1 \mathrm{C}), 153.1,145.5,145.2$, $138.3,134.2,129.7,129.7,125.1,123.7,123.7,118.9,107.8,87.5,85.5,84.4,59.9,52.6,50.0$, 48.6, 41.6, 38.6, 32.9, 29.2, 26.7, 25.4, two carbons missing in the aromatic region. IR (KBr) $v$ 3441, 1544, 1501, 1593, 1223, $747 \mathrm{~cm}^{-1}$. HRMS (ESI) calcd for $\mathrm{C}_{36} \mathrm{H}_{33} \mathrm{FN}_{5} \mathrm{O}_{5}[\mathrm{M}+\mathrm{H}]^{+} 634.2460$, found 634.2451.

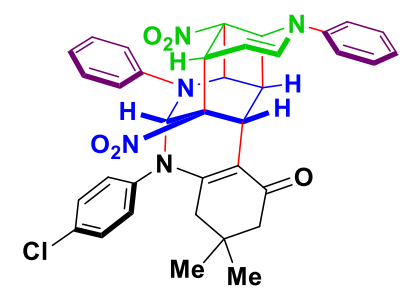

5-(4-chlorophenyl)-3,3-dimethyl-7b,13-dinitro-7,11-diphenyl-3,4,5,6,7,7a,7b,8,11,11a,11b,12-dod ecahydro-6,8,12-(epimethanetriyl)benzo[ $d]$ pyrido[3',2':3,4]cyclobuta[1,2-g][1,3]diazocin-1(2H)-o ne $(\mathbf{4 f})$

Yellow solid obtained by filtration of the precipitate; $78.5 \mathrm{mg}, 81 \%$ yield; $\mathrm{dr}>20: 1$; reaction time $=48 \mathrm{~h} ; \mathrm{mp} 171.6-172.3{ }^{\circ} \mathrm{C} ;{ }^{1} \mathrm{H}$ NMR $\left(400 \mathrm{MHz}, \mathrm{CDCl}_{3}\right) \delta 7.40(\mathrm{t}, J=8.0 \mathrm{~Hz}, 2 \mathrm{H}), 7.24(\mathrm{t}, J=8.0$ $\mathrm{Hz}, 3 \mathrm{H}), 7.21(\mathrm{~d}, J=8.0 \mathrm{~Hz}, 2 \mathrm{H}), 7.12(\mathrm{t}, J=8.0 \mathrm{~Hz}, 3 \mathrm{H}), 6.98(\mathrm{t}, J=8.0 \mathrm{~Hz}, 3 \mathrm{H}), 6.49(\mathrm{br}, 2 \mathrm{H})$, $6.03(\mathrm{~s}, 1 \mathrm{H}), 4.52\left(\mathrm{dd}, J_{I}=J_{2}=4.0 \mathrm{~Hz}, 1 \mathrm{H}\right), 4.36(\mathrm{t}, J=8.0 \mathrm{~Hz}, 1 \mathrm{H}), 4.17(\mathrm{~d}, J=8.0 \mathrm{~Hz}, 1 \mathrm{H})$, 
$4.07\left(\mathrm{dd}, J_{1}=J_{2}=4.0 \mathrm{~Hz}, 1 \mathrm{H}\right), 4.04(\mathrm{~s}, 1 \mathrm{H}), 3.26(\mathrm{t}, J=8.0 \mathrm{~Hz}, 1 \mathrm{H}), 2.27(\mathrm{t}, J=16.0 \mathrm{~Hz}, 2 \mathrm{H})$, 1.94 (s, 2H), 0.98 (s, 3H), 0.93 (s, 3H); ${ }^{13} \mathrm{C}$ NMR (100 MHz, $\left.\mathrm{CDCl}_{3}\right) \delta$ 194.3, 152.7, 145.4, 145.2, $140.8,134.2,133.7,129.8,129.7,129.6,128.9,125.3,124.0,123.7,118.8,108.3,87.4,85.5,84.4$, 59.9, 52.5, 50.0, 48.6, 41.7, 38.6, 33.1, 29.0, 26.9, 25.4. IR (KBr) v 3433, 1588, 1539, 1229, 746 $\mathrm{cm}^{-1}$. HRMS (ESI) calcd for $\mathrm{C}_{36} \mathrm{H}_{33} \mathrm{ClN}_{5} \mathrm{O}_{5}[\mathrm{M}+\mathrm{H}]^{+} 650.2165$, found 650.2171 .

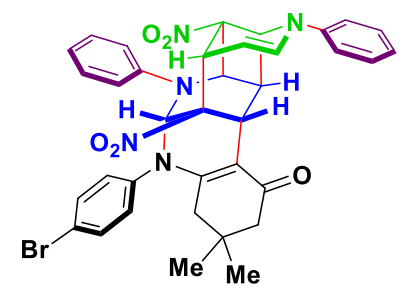

5-(4-bromophenyl)-3,3-dimethyl-7b,13-dinitro-7,11-diphenyl-3,4,5,6,7,7a,7b,8,11,11a,11b,12-dod ecahydro-6,8,12-(epimethanetriyl)benzo[ $d]$ pyrido[3',2':3,4]cyclobuta[1,2-g][1,3]diazocin-1(2H)-o ne $(4 \mathrm{~g})$

Yellow solid obtained by filtration of the precipitate; $84.6 \mathrm{mg}, 81 \%$ yield; $\mathrm{dr}>20: 1$; reaction time $=48 \mathrm{~h} ; \mathrm{mp} 177.9-178.6{ }^{\circ} \mathrm{C} ;{ }^{1} \mathrm{H}$ NMR $\left(400 \mathrm{MHz}, \mathrm{CDCl}_{3}\right) \delta 7.40(\mathrm{t}, J=8.0 \mathrm{~Hz}, 2 \mathrm{H}), 7.25(\mathrm{t}, J=8.0$ $\mathrm{Hz}, 4 \mathrm{H}), 7.21(\mathrm{~d}, J=8.0 \mathrm{~Hz}, 2 \mathrm{H}), 7.13(\mathrm{q}, J=8.0 \mathrm{~Hz}, 2 \mathrm{H}), 6.98(\mathrm{t}, J=8.0 \mathrm{~Hz}, 3 \mathrm{H}), 6.42(\mathrm{br}, 2 \mathrm{H})$, $6.02(\mathrm{~s}, 1 \mathrm{H}), 4.53\left(\mathrm{dd}, J_{I}=J_{2}=4.0 \mathrm{~Hz}, 1 \mathrm{H}\right), 4.36(\mathrm{t}, J=8.0 \mathrm{~Hz}, 1 \mathrm{H}), 4.17(\mathrm{~d}, J=8.0 \mathrm{~Hz}, 1 \mathrm{H})$, $4.07\left(\mathrm{dd}, J_{I}=J_{2}=4.0 \mathrm{~Hz}, 1 \mathrm{H}\right), 4.03(\mathrm{~s}, 1 \mathrm{H}), 3.26(\mathrm{t}, J=8.0 \mathrm{~Hz}, 1 \mathrm{H}), 2.27(\mathrm{t}, J=16.0 \mathrm{~Hz}, 2 \mathrm{H})$, 1.94 (s, 2H), 0.98 (s, 3H), 0.93 (s, 3H); $\left.{ }^{13} \mathrm{C} \mathrm{NMR} \mathrm{(100} \mathrm{MHz,} \mathrm{CDCl}_{3}\right) \delta$ 194.3, 152.6, 145.4, 145.2, $141.3,134.2,132.6,129.8,129.7,129.3,125.4,124.1,123.7,121.7,118.9,108.4,87.4,85.5,84.5$, $60.0,52.5,50.1,48.6,41.7,38.6,33.1,29.0,26.9,25.4 . \mathrm{IR}(\mathrm{KBr}) v 3431,1588,1536,1263,750$ $\mathrm{cm}^{-1}$. HRMS (ESI) calcd for $\mathrm{C}_{36} \mathrm{H}_{33} \mathrm{BrN}_{5} \mathrm{O}_{5}[\mathrm{M}+\mathrm{H}]^{+}$694.1660, found 694.1661.

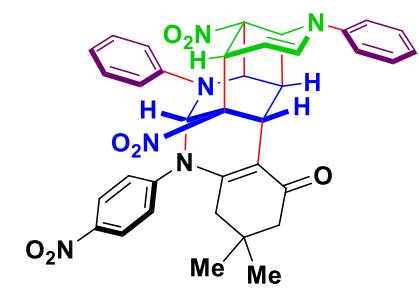

3,3-dimethyl-7b,13-dinitro-5-(4-nitrophenyl)-7,11-diphenyl-3,4,5,6,7,7a,7b,8,11,11a,11b,12-dodec ahydro-6,8,12-(epimethanetriyl)benzo[ $d]$ pyrido[3',2':3,4]cyclobuta[1,2-g][1,3]diazocin-1(2H)-one $(4 h)$

Yellow solid obtained by filtration of the precipitate; $69.2 \mathrm{mg}, 70 \%$ yield; $\mathrm{dr}>20: 1$; reaction time $=48 \mathrm{~h} ; \mathrm{mp} 182.3-183.1{ }^{\circ} \mathrm{C} ;{ }^{1} \mathrm{H}$ NMR $\left(400 \mathrm{MHz}, \mathrm{CDCl}_{3}\right) \delta 8.05(\mathrm{~d}, J=8.0 \mathrm{~Hz}, 2 \mathrm{H}), 7.41(\mathrm{t}, J=$ 
$8.0 \mathrm{~Hz}, 2 \mathrm{H}), 7.32(\mathrm{t}, J=8.0 \mathrm{~Hz}, 2 \mathrm{H}), 7.21(\mathrm{t}, J=8.0 \mathrm{~Hz}, 3 \mathrm{H}), 7.13(\mathrm{t}, J=8.0 \mathrm{~Hz}, 1 \mathrm{H}), 7.05(\mathrm{t}, J=$ $8.0 \mathrm{~Hz}, 2 \mathrm{H}), 6.99(\mathrm{~d}, J=8.0 \mathrm{~Hz}, 1 \mathrm{H}), 6.69(\mathrm{~d}, J=8.0 \mathrm{~Hz}, 2 \mathrm{H}), 6.01(\mathrm{~s}, 1 \mathrm{H}), 4.53\left(\mathrm{dd}, J_{l}=J_{2}=4.0\right.$ $\mathrm{Hz}, 1 \mathrm{H}), 4.35(\mathrm{t}, J=8.0 \mathrm{~Hz}, 1 \mathrm{H}), 4.13-4.09(\mathrm{~m}, 2 \mathrm{H}), 4.04(\mathrm{~s}, 1 \mathrm{H}), 3.26(\mathrm{t}, J=8.0 \mathrm{~Hz}, 1 \mathrm{H}), 2.34(\mathrm{t}$, $J=16.0 \mathrm{~Hz}, 2 \mathrm{H}), 2.16$ (d, $J=16.0 \mathrm{~Hz}, 1 \mathrm{H}), 1.95(\mathrm{~d}, J=16.0 \mathrm{~Hz}, 1 \mathrm{H}), 1.02(\mathrm{~s}, 3 \mathrm{H}), 0.99$ (s, 3H); ${ }^{13} \mathrm{C}$ NMR $\left(100 \mathrm{MHz}, \mathrm{CDCl}_{3}\right) \delta$ 194.6, 151.2, 147.8, 145.9, 145.2, 145.1, 134.3, 130.1, 129.7, 127.2, 126.3, 125.3, 125.0, 123.9, 118.9, 111.4, 87.3, 85.3, 84.6, 60.3, 52.3, 50.3, 48.4, 42.0, 38.8, 33.6, 28.4, 27.4, 25.7. IR (KBr) $v$ 3437, 1637, 1586, 1345, $737 \mathrm{~cm}^{-1}$. HRMS (ESI) calcd for $\mathrm{C}_{36} \mathrm{H}_{33} \mathrm{~N}_{6} \mathrm{O}_{7}[\mathrm{M}+\mathrm{H}]^{+}$661.2405, found 661.2387.

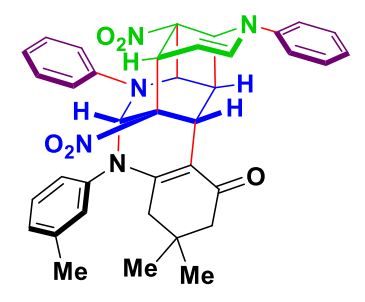

3,3-dimethyl-7b,13-dinitro-7,11-diphenyl-5-( $m$-tolyl)-3,4,5,6,7,7a,7b,8,11,11a,11b,12-dodecahydr o-6,8,12-(epimethanetriyl)benzo[ $d]$ pyrido[3',2':3,4]cyclobuta[1,2-g][1,3]diazocin-1(2H)-one (4i) Yellow solid obtained by column chromatography (petroleum ether/ethyl acetate $=10: 1$ to $5: 1$ ); $71.2 \mathrm{mg}, 75 \%$ yield; $\mathrm{dr}>20: 1$; reaction time $=48 \mathrm{~h}$; mp157.9-158.6 ${ }^{\circ} \mathrm{C} ;{ }^{1} \mathrm{H}$ NMR $(400 \mathrm{MHz}$, $\left.\mathrm{CDCl}_{3}\right) \delta 7.40(\mathrm{t}, J=8.0 \mathrm{~Hz}, 2 \mathrm{H}), 7.23(\mathrm{q}, J=8.0 \mathrm{~Hz}, 5 \mathrm{H}), 7.09(\mathrm{q}, J=8.0 \mathrm{~Hz}, 2 \mathrm{H}), 7.02-6.95(\mathrm{~m}$, 4H), $6.45(\mathrm{br}, 2 \mathrm{H}), 6.06(\mathrm{~s}, 1 \mathrm{H}), 4.54\left(\mathrm{dd}, J_{I}=J_{2}=4.0 \mathrm{~Hz}, 1 \mathrm{H}\right), 4.36(\mathrm{t}, J=8.0 \mathrm{~Hz}, 1 \mathrm{H}), 4.20(\mathrm{~d}, J$ $=8.0 \mathrm{~Hz}, 1 \mathrm{H}), 4.08\left(\mathrm{dd}, J_{I}=J_{2}=4.0 \mathrm{~Hz}, 1 \mathrm{H}\right), 4.04(\mathrm{~s}, 1 \mathrm{H}), 3.27(\mathrm{t}, J=8.0 \mathrm{~Hz}, 1 \mathrm{H}), 2.31-1.92(\mathrm{~m}$, 7H), 0.97 (s, 3H), 0.93 (s, 3H); ${ }^{13} \mathrm{C}$ NMR (100 MHz, $\left.\mathrm{CDCl}_{3}\right) \delta$ 194.2, 153.4, 145.6, 145.2, 142.1, 134.1, 129.7, 129.7, 129.5, 128.5, 124.9, 123.9, 123.8, 123.6, 118.8, 107.4, 87.4, 85.6, 84.5, 59.9, $52.6,50.1,48.7,41.6,38.6,33.0,29.1,26.8,25.4,21.1$, two carbons missing in the aromatic region. IR (KBr) $v 3443,2954,1587,1543,746 \mathrm{~cm}^{-1}$. HRMS (ESI) calcd for $\mathrm{C}_{37} \mathrm{H}_{36} \mathrm{~N}_{5} \mathrm{O}_{5}[\mathrm{M}+\mathrm{H}]^{+}$ 630.2711, found 630.2699.

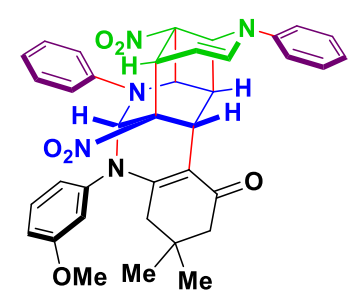


5-(3-methoxyphenyl)-3,3-dimethyl-7b,13-dinitro-7,11-diphenyl-3,4,5,6,7,7a,7b,8,11,11a,11b,12-d odecahydro-6,8,12-(epimethanetriyl)benzo[ $[d]$ pyrido[3',2':3,4] cyclobuta[1,2-g][1,3]diazocin-1(2H) -one $(\mathbf{4 j})$

Yellow solid obtained by column chromatography (petroleum ether/ethyl acetate $=10: 1$ to $5: 1$ ); $74.1 \mathrm{mg}, 77 \%$ yield; dr $>20: 1$; reaction time $=48 \mathrm{~h} ; \mathrm{mp} 165.1-165.7{ }^{\circ} \mathrm{C} ;{ }^{1} \mathrm{H}$ NMR $(400 \mathrm{MHz}$, $\left.\mathrm{CDCl}_{3}\right) \delta 7.39(\mathrm{t}, J=8.0 \mathrm{~Hz}, 2 \mathrm{H}), 7.27-7.19(\mathrm{~m}, 4 \mathrm{H}), 7.10(\mathrm{t}, J=8.0 \mathrm{~Hz}, 3 \mathrm{H}), 6.99\left(\mathrm{dd}, J_{l}=J_{2}=\right.$ $8.0 \mathrm{~Hz}, 3 \mathrm{H}), 6.76\left(\mathrm{dd}, J_{l}=J_{2}=4.0 \mathrm{~Hz}, 1 \mathrm{H}\right), 6.21(\mathrm{br}, 2 \mathrm{H}), 6.06(\mathrm{~s}, 1 \mathrm{H}), 4.53\left(\mathrm{dd}, J_{l}=J_{2}=4.0 \mathrm{~Hz}\right.$, $1 \mathrm{H}), 4.35(\mathrm{t}, J=8.0 \mathrm{~Hz}, 1 \mathrm{H}), 4.18(\mathrm{~d}, J=8.0 \mathrm{~Hz}, 1 \mathrm{H}), 4.08\left(\mathrm{dd}, J_{l}=J_{2}=4.0 \mathrm{~Hz}, 1 \mathrm{H}\right), 4.03(\mathrm{~s}, 1 \mathrm{H})$, $3.53(\mathrm{br}, 3 \mathrm{H}), 3.26(\mathrm{t}, J=8.0 \mathrm{~Hz}, 1 \mathrm{H}), 2.32-2.23(\mathrm{~m}, 2 \mathrm{H}), 2.07-1.97(\mathrm{~m}, 2 \mathrm{H}), 0.98(\mathrm{~s}, 3 \mathrm{H}), 0.94(\mathrm{~s}$, $3 \mathrm{H}) ;{ }^{13} \mathrm{C}$ NMR (100 MHz, $\left.\mathrm{CDCl}_{3}\right) \delta 194.3,160.2,153.3,145.6,145.2,143.2,134.0,130.0,129.7$, 129.6, 125.1, 125.1, 124.1, 123.6, 118.8, 114.3, 112.5, 107.9, 87.4, 85.6, 84.5, 60.0, 55.1, 52.5, 50.1, 48.5, 41.5, 38.6, 33.0, 29.0, 26.9, 25.4. IR (KBr) $v 3445,2953,1591,1493,754 \mathrm{~cm}^{-1}$. HRMS (ESI) calcd for $\mathrm{C}_{37} \mathrm{H}_{36} \mathrm{~N}_{5} \mathrm{O}_{6}[\mathrm{M}+\mathrm{H}]^{+} 646.2660$, found 646.2663.

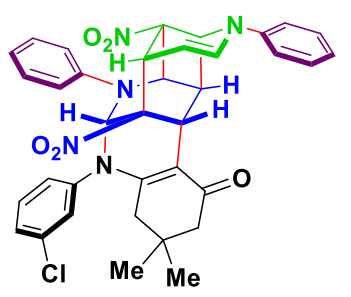

5-(3-chlorophenyl)-3,3-dimethyl-7b,13-dinitro-7,11-diphenyl-3,4,5,6,7,7a,7b, 8,11,11a,11b,12-dod ecahydro-6,8,12-(epimethanetriyl)benzo[ $d]$ pyrido[3',2':3,4]cyclobuta[1,2-g][1,3]diazocin-1(2H)-o ne $(\mathbf{4 k})$

Yellow solid obtained by column chromatography (petroleum ether/ethyl acetate $=10: 1$ to $5: 1$ ); $69.3 \mathrm{mg}, 71 \%$ yield; $\mathrm{dr}>20: 1$; reaction time $=48 \mathrm{~h}$; mp 168.2-169.0 ${ }^{\circ} \mathrm{C} ;{ }^{1} \mathrm{H}$ NMR $(300 \mathrm{MHz}$, $\left.\mathrm{CDCl}_{3}\right) \delta 7.39(\mathrm{t}, J=6.0 \mathrm{~Hz}, 2 \mathrm{H}), 7.28-7.17(\mathrm{~m}, 5 \mathrm{H}), 7.11(\mathrm{q}, J=6.0 \mathrm{~Hz}, 3 \mathrm{H}), 6.98(\mathrm{~d}, J=6.0 \mathrm{~Hz}$, 3H), $6.51(\mathrm{br}, 2 \mathrm{H}), 6.05(\mathrm{~s}, 1 \mathrm{H}), 4.53(\mathrm{~d}, J=3.0 \mathrm{~Hz}, 1 \mathrm{H}), 4.34(\mathrm{t}, J=6.0 \mathrm{~Hz}, 1 \mathrm{H}), 4.17$ (d, $J=6.0$ $\mathrm{Hz}, 1 \mathrm{H}), 4.07$ (d, J = 3.0 Hz, 1H), $4.04(\mathrm{~s}, 1 \mathrm{H}), 3.26(\mathrm{t}, J=6.0 \mathrm{~Hz}, 1 \mathrm{H}), 2.28(\mathrm{~s}, 2 \mathrm{H}), 1.96(\mathrm{~s}, 2 \mathrm{H})$, 0.98 (s, 3H), 0.93 (s, 3H); ${ }^{13} \mathrm{C}$ NMR (75 MHz, $\left.\mathrm{CDCl}_{3}\right) \delta 194.3,152.5,145.2,145.1,143.2,134.7$, 134.1, 130.2, 129.8, 129.7, 129.6, 128.0, 127.9, 125.4, 124.1, 123.6, 118.7, 108.4, 87.3, 85.4, 84.4, 59.9, 52.4, 50.0, 48.5, 41.6, 38.6, 33.0, 28.9, 26.8, 25.4. IR (KBr) v 3438, 2954, 1587, 1545, 747 $\mathrm{cm}^{-1}$. HRMS (ESI) calcd for $\mathrm{C}_{36} \mathrm{H}_{33} \mathrm{ClN}_{5} \mathrm{O}_{5}[\mathrm{M}+\mathrm{H}]^{+}$650.2165, found 650.2152. 


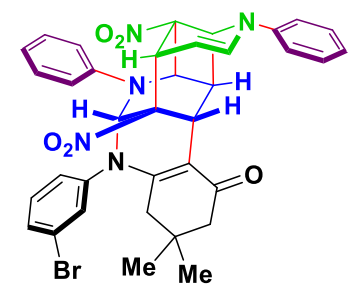

5-(3-bromophenyl)-3,3-dimethyl-7b,13-dinitro-7,11-diphenyl-3,4,5,6,7,7a,7b,8,11,11a,11b,12-dod ecahydro-6,8,12-(epimethanetriyl)benzo[ $d]$ pyrido[3',2':3,4] cyclobuta[1,2-g][1,3]diazocin-1(2H)-o ne $(\mathbf{4 I})$

Yellow solid obtained by column chromatography (petroleum ether/ethyl acetate $=10: 1$ to $5: 1$ ); $79.6 \mathrm{mg}, 76 \%$ yield; $\mathrm{dr}>20: 1$; reaction time $=48 \mathrm{~h}$; mp 176.3-176.9 ${ }^{\circ} \mathrm{C} ;{ }^{1} \mathrm{H}$ NMR $(400 \mathrm{MHz}$, $\left.\mathrm{CDCl}_{3}\right) \delta 7.31(\mathrm{t}, J=8.0 \mathrm{~Hz}, 2 \mathrm{H}), 7.25(\mathrm{~d}, J=8.0 \mathrm{~Hz}, 1 \mathrm{H}), 7.19(\mathrm{t}, J=8.0 \mathrm{~Hz}, 2 \mathrm{H}), 7.12(\mathrm{~d}, J=$ $8.0 \mathrm{~Hz}, 2 \mathrm{H}), 7.03\left(\mathrm{dd}, J_{l}=J_{2}=8.0 \mathrm{~Hz}, 3 \mathrm{H}\right), 6.90(\mathrm{~d}, J=8.0 \mathrm{~Hz}, 3 \mathrm{H}), 6.50(\mathrm{br}, 2 \mathrm{H}), 5.96(\mathrm{~s}, 1 \mathrm{H})$, $4.45(\mathrm{~d}, J=4.0 \mathrm{~Hz}, 1 \mathrm{H}), 4.26(\mathrm{t}, J=8.0 \mathrm{~Hz}, 1 \mathrm{H}), 4.08(\mathrm{~d}, J=8.0 \mathrm{~Hz}, 1 \mathrm{H}), 3.99(\mathrm{~d}, J=4.0 \mathrm{~Hz}, 1 \mathrm{H})$, $3.95(\mathrm{~s}, 1 \mathrm{H}), 3.17$ (t, $J=8.0 \mathrm{~Hz}, 1 \mathrm{H}), 2.20$ (t, $J=16.0 \mathrm{~Hz}, 2 \mathrm{H}), 1.88(\mathrm{~s}, 2 \mathrm{H}), 0.90$ (s, 3H), 0.85 (s, $3 \mathrm{H}) ;{ }^{13} \mathrm{C}$ NMR (100 MHz, $\left.\mathrm{CDCl}_{3}\right) \delta 194.3,152.5,145.2,145.1,143.4,134.1,130.9,130.5,129.8$, 129.6, 125.4, 124.2, 123.6, 122.6, 118.8, 108.4, 87.3, 85.4, 84.4, 59.9, 52.4, 50.0, 48.6, 41.6, 38.6, 33.1, 28.9, 26.9, 25.4, two carbons missing in the aromatic region. IR $(\mathrm{KBr}) v 3443,2957,1590$, 1544, $744 \mathrm{~cm}^{-1}$. HRMS (ESI) calcd for $\mathrm{C}_{36} \mathrm{H}_{33} \mathrm{BrN}_{5} \mathrm{O}_{5}[\mathrm{M}+\mathrm{H}]^{+}$694.1660, found 694.1662.

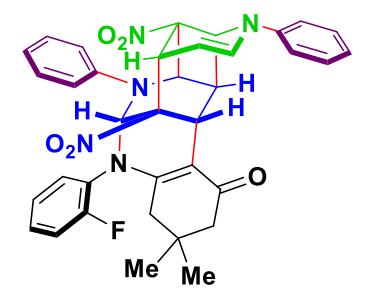

5-(2-fluorophenyl)-3,3-dimethyl-7b,13-dinitro-7,11-diphenyl-3,4,5,6,7,7a,7b,8,11,11a,11b,12-dod ecahydro-6,8,12-(epimethanetriyl)benzo[ $d]$ pyrido[3',2':3,4]cyclobuta[1,2-g][1,3] diazocin-1(2H)-o ne $(\mathbf{4 m})$

Yellow solid obtained by column chromatography (petroleum ether/ethyl acetate $=10: 1$ to 5:1); $67.3 \mathrm{mg}, 71 \%$ yield; $\mathrm{dr}=1.4: 1$; reaction time $=48 \mathrm{~h}$; mp 173.8-174.1 ${ }^{\circ} \mathrm{C} ;{ }^{1} \mathrm{H}$ NMR $(400 \mathrm{MHz}$, $\left.\mathrm{CDCl}_{3}\right) \delta 7.40(\mathrm{t}, J=8.0 \mathrm{~Hz}, 2 \mathrm{H}), 7.33(\mathrm{t}, J=8.0 \mathrm{~Hz}, 1 \mathrm{H}), 7.21(\mathrm{~d}, J=8.0 \mathrm{~Hz}, 4 \mathrm{H}), 7.13-6.97(\mathrm{~m}$, $5 \mathrm{H}), 6.88(\mathrm{t}, J=8.0 \mathrm{~Hz}, 1 \mathrm{H}), 6.76(\mathrm{t}, J=8.0 \mathrm{~Hz}, 2 \mathrm{H}), 6.26(\mathrm{~s}, 1 \mathrm{H}), 4.57\left(\mathrm{dd}, J_{l}=J_{2}=4.0 \mathrm{~Hz}, 1 \mathrm{H}\right)$, 4.40-4.33 (m, 2H), 4.10-4.02 (m, 2H), 3.27 (q, $J=8.0 \mathrm{~Hz}, 1 \mathrm{H}), 2.30(\mathrm{t}, J=16.0 \mathrm{~Hz}, 2 \mathrm{H}), 2.08$ (dd, $\left.J_{l}=J_{2}=16.0 \mathrm{~Hz}, 1 \mathrm{H}\right), 1.82\left(\mathrm{dd}, J_{I}=J_{2}=16.0 \mathrm{~Hz}, 1 \mathrm{H}\right), 0.98(\mathrm{~d}, J=8.0 \mathrm{~Hz}, 3 \mathrm{H}), 0.91(\mathrm{~d}, J=32.0$ 
$\mathrm{Hz}, 3 \mathrm{H}) ;{ }^{13} \mathrm{C}$ NMR $\left(100 \mathrm{MHz}, \mathrm{CDCl}_{3}\right) \delta 194.5,159.5(\mathrm{~d}, J=66.0 \mathrm{~Hz}, 1 \mathrm{C}), 153.5,145.7,145.2$, $134.4,130.3,130.0,129.7,129.2,126.0,125.5,123.8,123.2,119.9,119.0,116.6(\mathrm{~d}, J=20.0 \mathrm{~Hz}$, 1C), 106.4, 88.0, 85.4, 84.1, 58.8, 52.9, 49.9, 48.5, 39.6, 38.4, 32.7, 30.2, 25.7, 25.0. IR (KBr) $v$ $3439,2954,1592,1543,756 \mathrm{~cm}^{-1}$. HRMS (ESI) calcd for $\mathrm{C}_{36} \mathrm{H}_{33} \mathrm{FN}_{5} \mathrm{O}_{5}[\mathrm{M}+\mathrm{H}]^{+} 634.2460$, found 634.2445 .

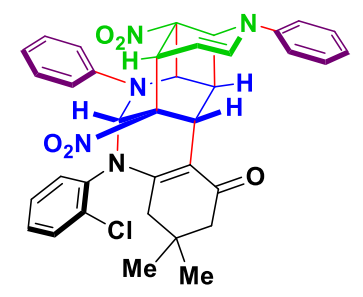

5-(2-chlorophenyl)-3,3-dimethyl-7b,13-dinitro-7,11-diphenyl-3,4,5,6,7,7a,7b,8,11,11a,11b,12-dod ecahydro-6,8,12-(epimethanetriyl)benzo[ $d]$ pyrido[3',2':3,4]cyclobuta[1,2-g][1,3] diazocin-1(2H)-o ne $(4 n)$

Yellow solid obtained by column chromatography (petroleum ether/ethyl acetate $=10: 1$ to $5: 1$ ); $68.5 \mathrm{mg}, 70 \%$ yield; $\mathrm{dr}=1.2: 1$; reaction time $=48 \mathrm{~h} ; \mathrm{mp} 166.8-167.1{ }^{\circ} \mathrm{C} ;{ }^{1} \mathrm{H}$ NMR $(400 \mathrm{MHz}$, $\left.\mathrm{CDCl}_{3}\right) \delta 7.35-7.30(\mathrm{~m}, 2 \mathrm{H}), 7.25(\mathrm{t}, J=8.0 \mathrm{~Hz}, 1 \mathrm{H}), 7.20-7.10(\mathrm{~m}, 4 \mathrm{H}), 7.06-6.95(\mathrm{~m}, 4 \mathrm{H}), 6.91$ $\left(\mathrm{dd}, J_{I}=J_{2}=4.0 \mathrm{~Hz}, 1 \mathrm{H}\right), 6.81(\mathrm{t}, J=8.0 \mathrm{~Hz}, 1 \mathrm{H}), 6.69(\mathrm{t}, J=8.0 \mathrm{~Hz}, 2 \mathrm{H}), 6.18(\mathrm{~d}, J=4.0 \mathrm{~Hz}$, $1 \mathrm{H}), 4.49\left(\mathrm{dd}, J_{l}=J_{2}=4.0 \mathrm{~Hz}, 1 \mathrm{H}\right), 4.33-4.26(\mathrm{~m}, 2 \mathrm{H}), 4.04-3.94(\mathrm{~m}, 2 \mathrm{H}), 3.19(\mathrm{t}, J=8.0 \mathrm{~Hz}$, $1 \mathrm{H}), 2.22(\mathrm{t}, J=20.0 \mathrm{~Hz}, 2 \mathrm{H}), 2.14-1.89(\mathrm{~m}, 1 \mathrm{H}), 1.75\left(\mathrm{dd}, J_{l}=J_{2}=16.0 \mathrm{~Hz}, 1 \mathrm{H}\right), 0.91(\mathrm{~d}, J=4.0$ $\mathrm{Hz}, 3 \mathrm{H}), 0.84(\mathrm{~d}, J=32.0 \mathrm{~Hz}, 3 \mathrm{H}) ;{ }^{13} \mathrm{C} \mathrm{NMR}\left(100 \mathrm{MHz}, \mathrm{CDCl}_{3}\right) \delta 194.5,153.4,145.7,145.2$, $134.4,130.3,130.0,129.7,129.1,125.5,124.6,123.8,123.1,119.9,119.0,116.5,116.3,106.4$, 88.0, 85.4, 84.1, 58.8, 52.9, 49.9, 48.5, 40.9, 38.4, 32.6, 30.1, 25.7, 25.0. IR (KBr) v 3441, 2956, 1593, 1498, $756 \mathrm{~cm}^{-1}$. HRMS (ESI) calcd for $\mathrm{C}_{36} \mathrm{H}_{33} \mathrm{ClN}_{5} \mathrm{O}_{5}[\mathrm{M}+\mathrm{H}]^{+} 650.2165$, found 650.2163 .

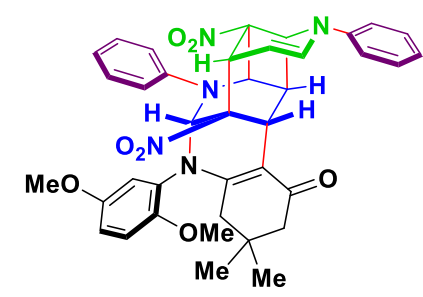

5-(2,5-dimethoxyphenyl)-3,3-dimethyl-7b,13-dinitro-7,11-diphenyl-3,4,5,6,7,7a,7b,8,11,11a,11b,1 2-dodecahydro-6,8,12-(epimethanetriyl)benzo[ $d]$ pyrido[3',2':3,4]cyclobuta[1,2-g][1,3]diazocin-1 (2H)-one $(\mathbf{4 o})$ 
Yellow solid obtained by column chromatography (petroleum ether/ethyl acetate $=10: 1$ to $5: 1$ ); $60.7 \mathrm{mg}, 61 \%$ yield; $\mathrm{dr}=1.8: 1$; reaction time $=48 \mathrm{~h} ; \mathrm{mp} 222.4-223.1{ }^{\circ} \mathrm{C} ;{ }^{1} \mathrm{H}$ NMR $(400 \mathrm{MHz}$, $\left.\mathrm{CDCl}_{3}\right) \delta$ 7.32-7.23 (m, 3H), 7.18-6.87 (m, 7H), 6.75-6.39 (m, 4H), $5.81(\mathrm{~s}, 1 \mathrm{H}), 4.48-4.41(\mathrm{~m}, 1 \mathrm{H})$, 4.28-4.22 (m, 1H), $3.97\left(\mathrm{dd}, J_{l}=J_{2}=8.0 \mathrm{~Hz}, 2 \mathrm{H}\right), 3.58(\mathrm{~s}, 3 \mathrm{H}), 3.24-3.16(\mathrm{~m}, 2 \mathrm{H}), 3.15(\mathrm{~s}, 3 \mathrm{H})$, 2.19 (s, 2H), 1.84 (s, 2H), 0.89 (s, 3H), 0.84 (s, 3H); $\left.{ }^{13} \mathrm{C} \mathrm{NMR} \mathrm{(100} \mathrm{MHz,} \mathrm{CDCl}_{3}\right) \delta$ 194.2, 155.0, 153.0, 149.2, 146.2, 145.2, 133.9, 130.7, 130.0, 129.6, 125.5, 123.5, 118.8, 118.7, 115.8, 114.2, $112.2,106.5,86.8,85.8,84.5,60.7,55.3,55.2,52.5,50.2,48.6,40.7,38.9,32.6,29.2,26.9,24.9$. IR (KBr) $v 3447,2959,1588,1502,1225,1036,750 \mathrm{~cm}^{-1}$. HRMS (ESI) calcd for $\mathrm{C}_{38} \mathrm{H}_{38} \mathrm{~N}_{5} \mathrm{O}_{7}$ $[\mathrm{M}+\mathrm{H}]^{+}$676.2766, found 676.2772 .

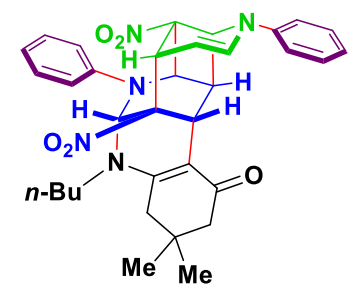

5-butyl-3,3-dimethyl-7b,13-dinitro-7,11-diphenyl-3,4,5,6,7,7a,7b,8,11,11a, 11b,12-dodecahydro-6, 8,12-(epimethanetriyl)benzo[d]pyrido[3',2':3,4]cyclobuta[1,2-g][1,3]diazocin-1(2H)-one $(\mathbf{4} \mathbf{p})$ Yellow solid obtained by column chromatography (petroleum ether/ethyl acetate $=10: 1$ to $5: 1$ ); $34.1 \mathrm{mg}, 38 \%$ yield; $\mathrm{dr}>20: 1$; reaction time $=48 \mathrm{~h}$; mp 199.1-199.8 ${ }^{\circ} \mathrm{C} ;{ }^{1} \mathrm{H}$ NMR $(400 \mathrm{MHz}$, $\left.\mathrm{CDCl}_{3}\right) \delta$ 7.39-7.31 (m, 4H), 7.19-7.04 (m, 6H), $6.95(\mathrm{~d}, J=8.0 \mathrm{~Hz}, 1 \mathrm{H}), 5.68(\mathrm{~s}, 1 \mathrm{H}), 4.49\left(\mathrm{dd}, J_{l}\right.$ $\left.=J_{2}=4.0 \mathrm{~Hz}, 1 \mathrm{H}\right), 4.35(\mathrm{t}, J=8.0 \mathrm{~Hz}, 1 \mathrm{H}), 4.11\left(\mathrm{dd}, J_{l}=J_{2}=4.0 \mathrm{~Hz}, 1 \mathrm{H}\right), 4.04(\mathrm{~d}, J=8.0 \mathrm{~Hz}$, 1H), $3.91(\mathrm{~s}, 1 \mathrm{H}), 3.27-3.17(\mathrm{~m}, 2 \mathrm{H}), 3.00-2.93(\mathrm{~m}, 1 \mathrm{H}), 2.29-2.14(\mathrm{~m}, 4 \mathrm{H}), 1.34-1.28(\mathrm{~m}, 1 \mathrm{H})$, 1.15-1.11 (m, 3H), 1.06 (s, 3H), 1.01 (s, 3H), 0.82 (t, $J=8.0 \mathrm{~Hz}, 3 \mathrm{H}) ;{ }^{13} \mathrm{C}$ NMR (100 MHz, $\left.\mathrm{CDCl}_{3}\right)$ $\delta 193.4,154.2,146.2,145.2,133.9,129.7,129.6,125.2,124.1,123.6,118.7,106.4,87.4,85.7$, 84.6, 59.9, 52.6, 51.2, 49.6, 48.9, 39.6, 38.5, 32.5, 31.4, 29.5, 27.0, 25.3, 19.7, 13.6. IR (KBr) $v$ $3453,2959,1632,1577,1225,752 \mathrm{~cm}^{-1}$. HRMS (ESI) calcd for $\mathrm{C}_{34} \mathrm{H}_{38} \mathrm{~N}_{5} \mathrm{O}_{5}[\mathrm{M}+\mathrm{H}]^{+} 596.2857$, found 596.2870.

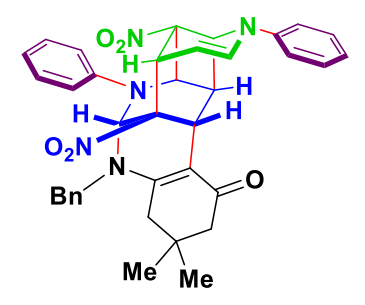


5-benzyl-3,3-dimethyl-7b,13-dinitro-7,11-diphenyl-3,4,5,6,7,7a,7b,8,11,11a,11b,12-dodecahydro6,8,12-(epimethanetriyl)benzo[d]pyrido[3',2':3,4]cyclobuta[1,2-g][1,3]diazocin-1(2H)-one $(\mathbf{4 q})$ Yellow solid obtained by column chromatography (petroleum ether/ethyl acetate $=10: 1$ to $5: 1$ ); $39.7 \mathrm{mg}, 42 \%$ yield; $\mathrm{dr}>20: 1$; reaction time $=48 \mathrm{~h}$; mp 214.6-215.1 ${ }^{\circ} \mathrm{C} ;{ }^{1} \mathrm{H}$ NMR $(400 \mathrm{MHz}$, $\left.\mathrm{CDCl}_{3}\right) \delta 7.38(\mathrm{t}, J=8.0 \mathrm{~Hz}, 2 \mathrm{H}), 7.31(\mathrm{t}, J=8.0 \mathrm{~Hz}, 2 \mathrm{H}), 7.27-7.25(\mathrm{~m}, 3 \mathrm{H}), 7.17(\mathrm{t}, J=8.0 \mathrm{~Hz}$, 3H), $7.10(\mathrm{q}, J=8.0 \mathrm{~Hz}, 3 \mathrm{H}), 6.94(\mathrm{~d}, J=8.0 \mathrm{~Hz}, 1 \mathrm{H}), 6.83\left(\mathrm{dd}, J_{I}=J_{2}=4.0 \mathrm{~Hz}, 2 \mathrm{H}\right), 5.76(\mathrm{~s}$, $1 \mathrm{H}), 4.48\left(\mathrm{dd}, J_{l}=J_{2}=4.0 \mathrm{~Hz}, 1 \mathrm{H}\right), 4.44(\mathrm{~s}, 1 \mathrm{H}), 4.29(\mathrm{t}, J=8.0 \mathrm{~Hz}, 1 \mathrm{H}), 4.11(\mathrm{~d}, J=16.0 \mathrm{~Hz}$, 1H), 4.06-4.03 (m, 2H), 3.97 (s, 1H), $3.20(\mathrm{t}, J=8.0 \mathrm{~Hz}, 1 \mathrm{H}), 2.31-2.22(\mathrm{~m}, 4 \mathrm{H}), 1.03(\mathrm{~s}, 3 \mathrm{H})$, $0.99(\mathrm{~s}, 3 \mathrm{H}) ;{ }^{13} \mathrm{C} \mathrm{NMR}\left(100 \mathrm{MHz}, \mathrm{CDCl}_{3}\right) \delta 193.7,154.5,146.4,145.2,135.8,134.1,129.9,129.7$, $128.8,127.9,126.4,125.5,124.5,123.6,118.8,106.9,87.0,85.6,84.4,60.5,53.9,52.6,49.6,49.0$, 40.2, 38.5, 32.7, 29.6, 26.8, 25.2. IR (KBr) v 3440, 2945, 1633, 1588, 1543, $750 \mathrm{~cm}^{-1}$. HRMS (ESI) calcd for $\mathrm{C}_{37} \mathrm{H}_{36} \mathrm{~N}_{5} \mathrm{O}_{5}[\mathrm{M}+\mathrm{H}]^{+} 630.2711$, found 630.2713 .

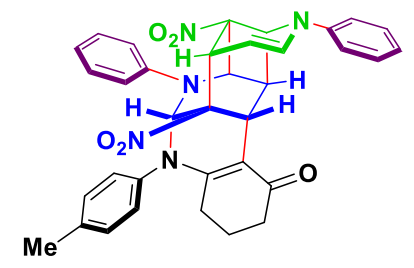

7b,13-dinitro-7,11-diphenyl-5-(p-tolyl)-3,4,5,6,7,7a,7b,8,11,11a,11b,12-dodecahydro-6,8,12-(epim ethanetriyl)benzo[ $[d]$ pyrido[3',2':3,4]cyclobuta[1,2-g][1,3]diazocin-1(2H)-one $(\mathbf{4 r})$

Yellow solid obtained by column chromatography (petroleum ether/ethyl acetate $=10: 1$ to $5: 1$ ); $61.2 \mathrm{mg}, 68 \%$ yield; $\mathrm{dr}>20: 1$; reaction time $=48 \mathrm{~h}$; mp 165.4-166.2 ${ }^{\circ} \mathrm{C} ;{ }^{1} \mathrm{H}$ NMR $(400 \mathrm{MHz}$, $\left.\mathrm{CDCl}_{3}\right) \delta 7.38(\mathrm{t}, J=8.0 \mathrm{~Hz}, 2 \mathrm{H}), 7.21-7.17(\mathrm{~m}, 4 \mathrm{H}), 7.09(\mathrm{t}, J=8.0 \mathrm{~Hz}, 1 \mathrm{H}), 7.03(\mathrm{t}, J=8.0 \mathrm{~Hz}$, $1 \mathrm{H}), 6.94\left(\mathrm{dd}, J_{l}=J_{2}=8.0 \mathrm{~Hz}, 4 \mathrm{H}\right), 6.50(\mathrm{br}, 2 \mathrm{H}), 6.07(\mathrm{~s}, 1 \mathrm{H}), 4.53(\mathrm{~d}, J=4.0 \mathrm{~Hz}, 1 \mathrm{H}), 4.33(\mathrm{t}, J$ $=8.0 \mathrm{~Hz}, 1 \mathrm{H}), 4.24(\mathrm{~d}, J=4.0 \mathrm{~Hz}, 1 \mathrm{H}), 4.04(\mathrm{~s}, 2 \mathrm{H}), 3.28(\mathrm{t}, J=8.0 \mathrm{~Hz}, 1 \mathrm{H}), 2.43-2.30(\mathrm{~m}, 3 \mathrm{H})$, $2.27(\mathrm{~s}, 3 \mathrm{H}), 2.07(\mathrm{t}, J=8.0 \mathrm{~Hz}, 2 \mathrm{H}), 1.87-1.75(\mathrm{~m}, 2 \mathrm{H}) ;{ }^{13} \mathrm{C} \mathrm{NMR}\left(100 \mathrm{MHz}, \mathrm{CDCl}_{3}\right) \delta 194.4$, 155.3, 145.4, 145.1, 139.6, 137.9, 133.9, 129.8, 129.6, 129.4, 127.5, 124.4, 123.5, 122.8, 118.7, 108.0, 87.5, 85.6, 84.2, 76.0, 59.3, 52.6, 48.5, 38.5, 36.3, 28.1, 25.3, 21.5, 20.9. IR (KBr) v 3438, 1572, 1502, $739 \mathrm{~cm}^{-1}$. HRMS (ESI) calcd for $\mathrm{C}_{35} \mathrm{H}_{32} \mathrm{~N}_{5} \mathrm{O}_{5}[\mathrm{M}+\mathrm{H}]^{+} 602.2398$, found 602.2386.

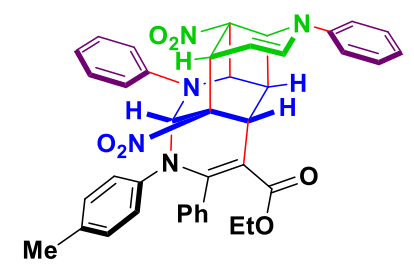


ethyl 10a,11-dinitro-1,4,7-triphenyl-3-(p-tolyl)-1,2,3,6,6a,6b,7,10,10a,10b-decahydro-2,6,10-(epi methanetriyl)pyrido[2',3':3,4]cyclobuta[1,2-d][1,3]diazocine-5-carboxylate (4s)

Yellow solid obtained by column chromatography (petroleum ether/ethyl acetate $=10: 1$ to $5: 1$ ); $57.5 \mathrm{mg}, 56 \%$ yield; $\mathrm{dr}>20: 1$; reaction time $=48 \mathrm{~h}$; mp 232.6-233.4 ${ }^{\circ} \mathrm{C} ;{ }^{1} \mathrm{H}$ NMR $(400 \mathrm{MHz}$, $\left.\mathrm{CDCl}_{3}\right) \delta 7.42(\mathrm{t}, J=8.0 \mathrm{~Hz}, 2 \mathrm{H}), 7.37(\mathrm{t}, J=8.0 \mathrm{~Hz}, 2 \mathrm{H}), 7.27(\mathrm{t}, J=8.0 \mathrm{~Hz}, 3 \mathrm{H}), 7.12-7.00(\mathrm{~m}$, $7 \mathrm{H}), 6.93(\mathrm{br}, 2 \mathrm{H}), 6.66(\mathrm{~d}, J=8.0 \mathrm{~Hz}, 2 \mathrm{H}), 6.21(\mathrm{~d}, J=8.0 \mathrm{~Hz}, 2 \mathrm{H}), 6.00(\mathrm{~s}, 1 \mathrm{H}), 4.66\left(\mathrm{dd}, J_{l}=\right.$ $\left.J_{2}=4.0 \mathrm{~Hz}, 1 \mathrm{H}\right), 4.46(\mathrm{t}, J=8.0 \mathrm{~Hz}, 1 \mathrm{H}), 4.19\left(\mathrm{dd}, J_{1}=J_{2}=4.0 \mathrm{~Hz}, 1 \mathrm{H}\right), 4.16(\mathrm{~d}, J=8.0 \mathrm{~Hz}, 1 \mathrm{H})$, $4.10(\mathrm{~s}, 1 \mathrm{H}), 3.95-3.82(\mathrm{~m}, 2 \mathrm{H}), 3.24(\mathrm{t}, J=8.0 \mathrm{~Hz}, 1 \mathrm{H}), 2.07(\mathrm{~s}, 3 \mathrm{H}), 0.80(\mathrm{t}, J=8.0 \mathrm{~Hz}, 3 \mathrm{H}) ;{ }^{13} \mathrm{C}$ NMR $\left(100 \mathrm{MHz}, \mathrm{CDCl}_{3}\right) \delta 166.5,150.5,145.5,144.7,141.4,136.3,135.6,133.2,129.9,129.7$, $129.2,127.9,127.3,126.9,126.2,126.1,123.1,117.6,103.1,87.2,87.0,85.1,78.0,60.1,59.7$, 51.7, 48.6, 38.6, 28.7, 20.7, 13.8, one carbon missing in the aromatic region. IR (KBr) $v$ 3450, 2922, 1681, 1594, 1544, 1233, 1121, $744 \mathrm{~cm}^{-1}$. HRMS (ESI) calcd for $\mathrm{C}_{40} \mathrm{H}_{36} \mathrm{~N}_{5} \mathrm{O}_{6}[\mathrm{M}+\mathrm{H}]^{+}$ 682.2660 , found 682.2660 .

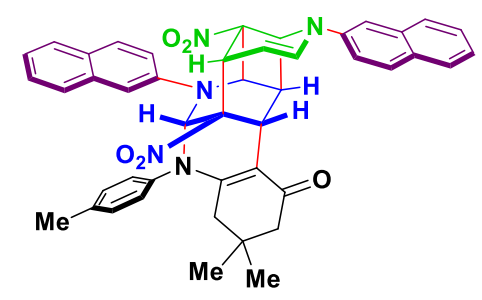

3,3-dimethyl-7,11-di(naphthalen-2-yl)-7b,13-dinitro-5-(p-tolyl)-3,4,5,6,7,7a,7b,8,11,11a,11b,12-d odecahydro-6,8,12-(epimethanetriyl)benzo[ $d]$ pyrido[3',2':3,4]cyclobuta[1,2-g][1,3]diazocin-1(2H) -one $(\mathbf{4 t})$

Yellow solid obtained by column chromatography (petroleum ether/ethyl acetate $=10: 1$ to $5: 1$ ); $77.3 \mathrm{mg}, 71 \%$ yield; $\mathrm{dr}>20: 1$; reaction time $=48 \mathrm{~h}$; mp 246.2-246.7 ${ }^{\circ} \mathrm{C} ;{ }^{1} \mathrm{H}$ NMR $(400 \mathrm{MHz}$, $\left.\mathrm{CDCl}_{3}\right) \delta$ 7.83-7.63 (m, 5H), $7.52(\mathrm{~s}, 1 \mathrm{H}), 7.42-7.35(\mathrm{~m}, 5 \mathrm{H}), 7.18-7.05(\mathrm{~m}, 4 \mathrm{H}), 6.85(\mathrm{br}, 2 \mathrm{H})$, $6.44(\mathrm{br}, 2 \mathrm{H}), 6.17(\mathrm{~s}, 1 \mathrm{H}), 4.61(\mathrm{~s}, 1 \mathrm{H}), 4.38(\mathrm{~s}, 2 \mathrm{H}), 4.08(\mathrm{~s}, 2 \mathrm{H}), 3.35(\mathrm{~s}, 1 \mathrm{H}), 2.26-2.19(\mathrm{~m}, 5 \mathrm{H})$, $1.90(\mathrm{q}, J=16.0 \mathrm{~Hz}, 2 \mathrm{H}), 0.89(\mathrm{~s}, 3 \mathrm{H}), 0.85(\mathrm{~s}, 3 \mathrm{H}) ;{ }^{13} \mathrm{C} \mathrm{NMR}\left(100 \mathrm{MHz}, \mathrm{CDCl}_{3}\right) \delta$ 194.3, 153.7, 142.9, 142.8, 139.8, 138.0, 134.4, 134.1, 133.9, 130.4, 130.4, 130.0, 130.0, 129.8, 129.6, 127.6, $127.5,127.5,127.2,126.7,126.7,125.3,124.9,121.7,119.3,119.2,115.5,107.3,87.6,86.2,84.3$, 59.3, 53.0, 50.1, 48.5, 41.7, 38.6, 32.9, 29.4, 26.6, 25.5, 20.9. IR (KBr) v 3440, 2957, 1631, 1590, 1541, $743 \mathrm{~cm}^{-1}$. HRMS (ESI) calcd for $\mathrm{C}_{45} \mathrm{H}_{40} \mathrm{~N}_{5} \mathrm{O}_{5}[\mathrm{M}+\mathrm{H}]^{+} 730.3024$, found 730.3024 . 


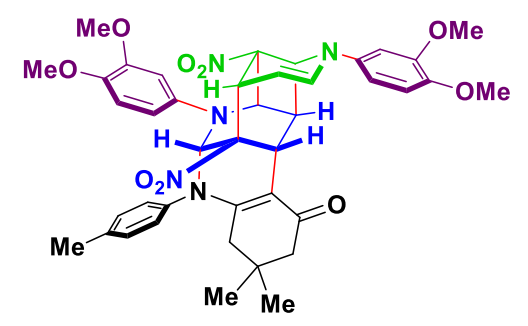

7,11-bis(3,4-dimethoxyphenyl)-3,3-dimethyl-7b,13-dinitro-5-(p-tolyl)-3,4,5,6,7,7a,7b,8,11,11a,11 b,12-dodecahydro-6,8,12-(epimethanetriyl)benzo[ $d]$ pyrido[3',2':3,4]cyclobuta[1,2-g][1,3]diazocin $-1(2 H)$-one $(4 \mathbf{u})$

Yellowoil obtained by column chromatography (petroleum ether/ethyl acetate $=10: 1$ to $5: 1$ ); 87.5 $\mathrm{mg}, 78 \%$ yield; $\mathrm{dr}>20: 1$; reaction time $=48 \mathrm{~h} ;{ }^{1} \mathrm{H}$ NMR $\left(400 \mathrm{MHz}, \mathrm{CDCl}_{3}\right) \delta 7.04\left(\mathrm{dd}, J_{l}=J_{2}=\right.$ $8.0 \mathrm{~Hz}, 1 \mathrm{H}), 6.95(\mathrm{br}, 1 \mathrm{H}), 6.87(\mathrm{~d}, J=8.0 \mathrm{~Hz}, 1 \mathrm{H}), 6.82\left(\mathrm{dd}, J_{I}=J_{2}=4.0 \mathrm{~Hz}, 2 \mathrm{H}\right), 6.79(\mathrm{~d}, J=$ $8.0 \mathrm{~Hz}, 1 \mathrm{H}), 6.74(\mathrm{~d}, J=8.0 \mathrm{~Hz}, 1 \mathrm{H}), 6.56\left(\mathrm{dd}, J_{1}=J_{2}=4.0 \mathrm{~Hz}, 2 \mathrm{H}\right), 6.42(\mathrm{br}, 2 \mathrm{H}), 5.81(\mathrm{~s}, 1 \mathrm{H})$, $4.39\left(\mathrm{dd}, J_{1}=J_{2}=4.0 \mathrm{~Hz}, 1 \mathrm{H}\right), 4.21(\mathrm{t}, J=8.0 \mathrm{~Hz}, 1 \mathrm{H}), 4.07\left(\mathrm{dd}, J_{I}=J_{2}=4.0 \mathrm{~Hz}, 1 \mathrm{H}\right), 4.00(\mathrm{~s}$, $1 \mathrm{H}), 3.97(\mathrm{~d}, J=8.0 \mathrm{~Hz}, 1 \mathrm{H}), 3.93(\mathrm{~s}, 3 \mathrm{H}), 3.87-3.81(\mathrm{~m}, 5 \mathrm{H}), 3.71(\mathrm{~s}, 3 \mathrm{H}), 3.17(\mathrm{t}, J=8.0 \mathrm{~Hz}$, 1H), $2.25(\mathrm{~s}, 5 \mathrm{H}), 2.17-1.90(\mathrm{~m}, 3 \mathrm{H}), 0.96(\mathrm{~s}, 3 \mathrm{H}), 0.93(\mathrm{~s}, 3 \mathrm{H}) ;{ }^{13} \mathrm{C}$ NMR $\left(100 \mathrm{MHz}, \mathrm{CDCl}_{3}\right) \delta$ 194.0, 153.4, 149.6, 149.6, 147.1, 146.2, 139.7, 139.5, 139.0, 137.5, 135.4, 129.9, 123.8, 117.7, 112.7, 111.9, 111.5, 109.4, 108.0, 105.3, 87.4, 84.5, 83.8, 78.3, 60.7, 56.1, 56.0, 55.7, 52.8, 50.1, 48.5, 41.6, 38.6, 33.0, 28.7, 27.1, 25.4, 20.1. IR (KBr) v 3443, 2950, 1586, 1514, 1238, 1024, 769 $\mathrm{cm}^{-1}$. HRMS (ESI) calcd for $\mathrm{C}_{41} \mathrm{H}_{44} \mathrm{~N}_{5} \mathrm{O}_{9}[\mathrm{M}+\mathrm{H}]^{+} 750.3134$, found 750.3147 .

\section{Experimental data for the formation of 6}

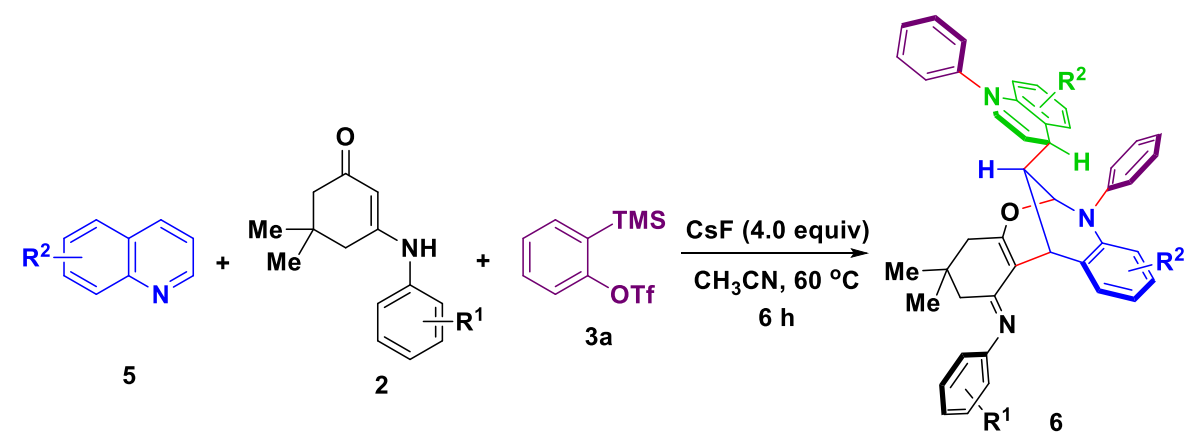

General procedure: To a $5.0 \mathrm{~mL}$ vial were successively added quinolines $5(0.30 \mathrm{mmol})$, enaminones $2(0.15 \mathrm{mmol})$, benzyne precursor $3 \mathbf{a}(0.60 \mathrm{mmol}), \mathrm{CsF}(0.60 \mathrm{mmol})$ and $1.0 \mathrm{~mL}$ of $\mathrm{CH}_{3} \mathrm{CN}$. The resulting mixture was stirred at $60{ }^{\circ} \mathrm{C}$ in oil bath for $6 \mathrm{~h}$. Upon completion of the reaction (monitoring by TLC), the products 6 were precipitated from the reaction mixtures and only a filtration was needed to purify them. (Note: The products were sensitive to acidic 
conditions, which could not be purified by silica gel column chromatography. They were liable to lose one molecule of quinoline to afford mono-quinoline bridged cyclic compounds 7.)

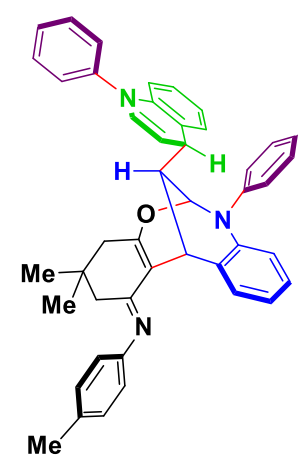

3,3-dimethyl-7-phenyl-13-(1-phenyl-1,4-dihydroquinolin-4-yl)- $N$-( $p$-tolyl)-2,3,4,6,7,12-hexahydro -1H-6,12-methanodibenzo[ $d, g][1,3]$ oxazocin-1-imine $(\mathbf{6 a})$

White solid obtained by filtration of the precipitate; $68.9 \mathrm{mg}, 72 \%$ yield; $\mathrm{dr}>20: 1$; reaction time $=$ $6 \mathrm{~h} ; \mathrm{mp} 195.5-196.1{ }^{\circ} \mathrm{C} ;{ }^{1} \mathrm{H}$ NMR $\left(400 \mathrm{MHz}, \mathrm{CDCl}_{3}\right) \delta 7.60(\mathrm{~d}, J=8.0 \mathrm{~Hz}, 1 \mathrm{H}), 7.44(\mathrm{t}, J=8.0$ $\mathrm{Hz}, 6 \mathrm{H}), 7.30(\mathrm{t}, J=8.0 \mathrm{~Hz}, 4 \mathrm{H}), 7.18(\mathrm{~d}, J=8.0 \mathrm{~Hz}, 1 \mathrm{H}), 7.09(\mathrm{~d}, J=8.0 \mathrm{~Hz}, 2 \mathrm{H}), 7.02-6.92(\mathrm{~m}$, $3 \mathrm{H}), 6.77(\mathrm{t}, J=8.0 \mathrm{~Hz}, 1 \mathrm{H}), 6.66-6.59(\mathrm{~m}, 5 \mathrm{H}), 5.44(\mathrm{~s}, 1 \mathrm{H}), 4.96(\mathrm{t}, J=8.0 \mathrm{~Hz}, 1 \mathrm{H}), 4.65(\mathrm{~s}, 1 \mathrm{H})$, $3.21(\mathrm{q}, J=8.0 \mathrm{~Hz}, 1 \mathrm{H}), 2.32(\mathrm{~s}, 3 \mathrm{H}), 2.29$ (d, $J=16.0 \mathrm{~Hz}, 1 \mathrm{H}), 2.12(\mathrm{~d}, J=16.0 \mathrm{~Hz}, 1 \mathrm{H}), 1.99$ (s, 2H), $1.89(\mathrm{~d}, J=16.0 \mathrm{~Hz}, 1 \mathrm{H}), 0.92(\mathrm{~s}, 3 \mathrm{H}), 0.81(\mathrm{~s}, 3 \mathrm{H}) ;{ }^{13} \mathrm{C} \mathrm{NMR}\left(100 \mathrm{MHz}, \mathrm{CDCl}_{3}\right) \delta 163.2$, 158.1, 150.0, 145.1, 143.7, 142.1, 141.9, 131.9, 131.3, 129.9, 129.8, 129.7, 129.4, 129.3, 127.8, 126.6, 126.6, 126.2, 126.2, 126.1, 122.7, 121.4, 119.8, 118.8, 114.4, 114.1, 113.4, 100.2, 84.5, 41.6, 41.6, 41.1, 36.1, 31.3, 29.0, 28.2, 27.5, 20.8. IR (KBr) v 3435, 2952, 1604, 1494, 1379, 753 $\mathrm{cm}^{-1}$. HRMS (ESI) calcd for $\mathrm{C}_{45} \mathrm{H}_{42} \mathrm{~N}_{3} \mathrm{O}[\mathrm{M}+\mathrm{H}]^{+} 640.3322$, found 640.3310 .

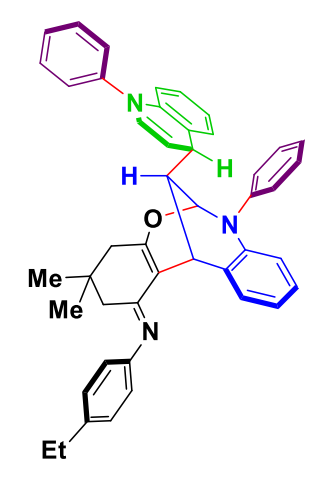

$N$-(4-ethylphenyl)-3,3-dimethyl-7-phenyl-13-(1-phenyl-1,4-dihydroquinolin-4-yl)-2,3,4,6,7,12-he xahydro-1 $H$-6,12-methanodibenzo $[d, g][1,3]$ oxazocin-1-imine $(\mathbf{6 b})$

White solid obtained by filtration of the precipitate; $78.8 \mathrm{mg}, 80 \%$ yield; $\mathrm{dr}>20: 1$; reaction time $=$ $6 \mathrm{~h} ; \mathrm{mp} 201.9-202.3{ }^{\circ} \mathrm{C} ;{ }^{1} \mathrm{H} \mathrm{NMR}\left(300 \mathrm{MHz}, \mathrm{CDCl}_{3}\right) \delta 7.60\left(\mathrm{dd}, J_{l}=J_{2}=3.0 \mathrm{~Hz}, 1 \mathrm{H}\right), 7.50-7.41$ 
(m, 5H), 7.33-7.29 (m, 6H), $7.18(\mathrm{~d}, J=3.0 \mathrm{~Hz}, 1 \mathrm{H}), 7.12(\mathrm{~d}, J=9.0 \mathrm{~Hz}, 2 \mathrm{H}), 7.04-6.91(\mathrm{~m}, 3 \mathrm{H})$, $6.77(\mathrm{t}, J=6.0 \mathrm{~Hz}, 1 \mathrm{H}), 6.66-6.61(\mathrm{~m}, 4 \mathrm{H}), 5.44(\mathrm{t}, J=3.0 \mathrm{~Hz}, 1 \mathrm{H}), 4.96(\mathrm{t}, J=6.0 \mathrm{~Hz}, 1 \mathrm{H}), 4.65$ $(\mathrm{s}, 1 \mathrm{H}), 3.21(\mathrm{q}, J=6.0 \mathrm{~Hz}, 1 \mathrm{H}), 2.63(\mathrm{q}, J=6.0 \mathrm{~Hz}, 2 \mathrm{H}), 2.28\left(\mathrm{tt}, J_{I}=J_{2}=3.0 \mathrm{~Hz}, 1 \mathrm{H}\right), 2.14(\mathrm{~d}, J$ $=15.0 \mathrm{~Hz}, 1 \mathrm{H}), 2.04(\mathrm{~s}, 2 \mathrm{H}), 1.90(\mathrm{~d}, J=18.0 \mathrm{~Hz}, 1 \mathrm{H}), 1.25(\mathrm{t}, J=6.0 \mathrm{~Hz}, 3 \mathrm{H}), 0.93(\mathrm{~s}, 3 \mathrm{H}), 0.82$ (s, 3H); ${ }^{13} \mathrm{C}$ NMR $\left(75 \mathrm{MHz}, \mathrm{CDCl}_{3}\right) \delta 163.2,158.0,150.2,145.1,143.7,142.1,141.9,137.9$, 131.9, 129.9, 129.8, 129.7, 129.4, 128.1, 127.8, 126.6, 126.6, 126.2, 126.1, 126.1, 122.7, 121.4, $119.8,118.8,114.4,114.1,113.4,100.2,84.5,41.6,41.5,41.2,36.1,31.3,29.0,28.3,28.2,27.5$, 15.8. IR (KBr) $v 3439,2959,2314,1607,1493,1380,754 \mathrm{~cm}^{-1}$. HRMS (ESI) calcd for $\mathrm{C}_{46} \mathrm{H}_{44} \mathrm{~N}_{3} \mathrm{O}[\mathrm{M}+\mathrm{H}]^{+} 654.3479$, found 654.3485 .

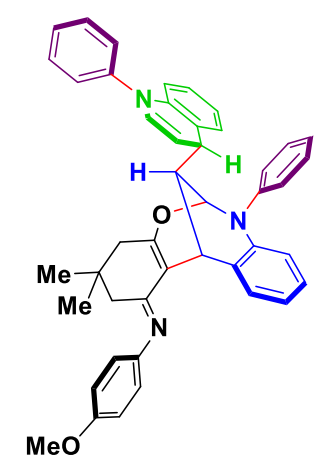

$N$-(4-methoxyphenyl)-3,3-dimethyl-7-phenyl-13-(1-phenyl-1,4-dihydroquinolin-4-yl)-2,3,4,6,7,12 -hexahydro-1H-6,12-methanodibenzo[d,g][1,3]oxazocin-1-imine (6c)

White solid obtained by filtration of the precipitate; $68.4 \mathrm{mg}, 70 \%$ yield; $\mathrm{dr}>20: 1$; reaction time $=$ $6 \mathrm{~h} ; \mathrm{mp} 221.2-221.9{ }^{\circ} \mathrm{C} ;{ }^{1} \mathrm{H}$ NMR $\left(400 \mathrm{MHz}, \mathrm{CDCl}_{3}\right) \delta 7.61(\mathrm{~d}, J=8.0 \mathrm{~Hz}, 1 \mathrm{H}), 7.45(\mathrm{q}, J=8.0$ $\mathrm{Hz}, 6 \mathrm{H}), 7.29(\mathrm{t}, J=8.0 \mathrm{~Hz}, 4 \mathrm{H}), 7.17(\mathrm{~d}, J=8.0 \mathrm{~Hz}, 1 \mathrm{H}), 7.02-6.92(\mathrm{~m}, 3 \mathrm{H}), 6.85(\mathrm{~d}, J=8.0 \mathrm{~Hz}$, 2H), $6.77(\mathrm{t}, J=8.0 \mathrm{~Hz}, 1 \mathrm{H}), 6.66-6.60(\mathrm{~m}, 5 \mathrm{H}), 5.44(\mathrm{~s}, 1 \mathrm{H}), 4.96(\mathrm{t}, J=8.0 \mathrm{~Hz}, 1 \mathrm{H}), 4.66(\mathrm{~s}, 1 \mathrm{H})$, $3.78(\mathrm{~s}, 3 \mathrm{H}), 3.22(\mathrm{q}, J=8.0 \mathrm{~Hz}, 1 \mathrm{H}), 2.29(\mathrm{~d}, J=8.0 \mathrm{~Hz}, 1 \mathrm{H}), 2.13(\mathrm{~d}, J=16.0 \mathrm{~Hz}, 1 \mathrm{H}), 2.04(\mathrm{~s}$, 2H), $1.89(\mathrm{~d}, J=16.0 \mathrm{~Hz}, 1 \mathrm{H}), 0.92(\mathrm{~s}, 3 \mathrm{H}), 0.81(\mathrm{~s}, 3 \mathrm{H}) ;{ }^{13} \mathrm{C}$ NMR $\left(100 \mathrm{MHz}, \mathrm{CDCl}_{3}\right) \delta 163.7$, 158.0, 155.2, 145.9, 145.1, 143.7, 142.1, 141.9, 131.9, 129.8, 129.8, 129.7, 129.4, 127.7, 126.6, 126.6, 126.2, 126.1, 126.1, 122.7, 121.4, 120.9, 118.8, 114.4, 114.1, 114.1, 113.4, 100.2, 84.5, 55.5, 41.6, 41.6, 41.2, 36.1, 31.3, 28.9, 28.2, 27.5. IR (KBr) v 3448, 2955, 1644, 1605, 1495, 1230 , $753 \mathrm{~cm}^{-1}$. HRMS (ESI) calcd for $\mathrm{C}_{45} \mathrm{H}_{42} \mathrm{~N}_{3} \mathrm{O}_{2}[\mathrm{M}+\mathrm{H}]^{+} 656.3272$, found 656.3290 . 


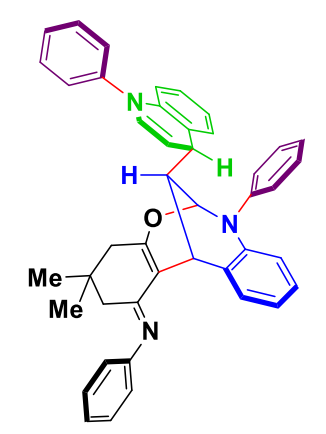

3,3-dimethyl-N,7-diphenyl-13-(1-phenyl-1,4-dihydroquinolin-4-yl)-2,3,4,6,7,12-hexahydro-1H-6, 12-methanodibenzo[d,g][1,3]oxazocin-1-imine (6d)

White solid obtained by filtration of the precipitate; $56.4 \mathrm{mg}, 60 \%$ yield; $\mathrm{dr}>20: 1$; reaction time $=$ 6 h; mp 198.7-199.3 ${ }^{\circ} \mathrm{C} ;{ }^{1} \mathrm{H}$ NMR $\left(400 \mathrm{MHz}, \mathrm{CDCl}_{3}\right) \delta 7.60(\mathrm{~d}, J=8.0 \mathrm{~Hz}, 1 \mathrm{H}), 7.48-7.41(\mathrm{~m}$, $6 \mathrm{H}), 7.27(\mathrm{q}, J=8.0 \mathrm{~Hz}, 6 \mathrm{H}), 7.17(\mathrm{~d}, J=4.0 \mathrm{~Hz}, 1 \mathrm{H}), 7.03-6.92(\mathrm{~m}, 4 \mathrm{H}), 6.78(\mathrm{t}, J=8.0 \mathrm{~Hz}, 1 \mathrm{H})$, 6.70-6.61 (m, 5H), $5.44(\mathrm{~s}, 1 \mathrm{H}), 4.96(\mathrm{t}, J=8.0 \mathrm{~Hz}, 1 \mathrm{H}), 4.65(\mathrm{~s}, 1 \mathrm{H}), 3.22(\mathrm{q}, J=8.0 \mathrm{~Hz}, 1 \mathrm{H})$, $2.29\left(\mathrm{dd}, J_{l}=J_{2}=4.0 \mathrm{~Hz}, 1 \mathrm{H}\right), 2.10(\mathrm{~d}, J=16.0 \mathrm{~Hz}, 1 \mathrm{H}), 2.04(\mathrm{~s}, 2 \mathrm{H}), 1.88(\mathrm{~d}, J=16.0 \mathrm{~Hz}, 1 \mathrm{H})$, $0.92(\mathrm{~s}, 3 \mathrm{H}), 0.81(\mathrm{~s}, 3 \mathrm{H}) ;{ }^{13} \mathrm{C}$ NMR $\left(100 \mathrm{MHz}, \mathrm{CDCl}_{3}\right) \delta 163.2,158.4,152.7,145.1,143.7,142.1$, $141.9,132.0,129.8,129.8,129.7,129.4,128.7,127.8,126.7,126.6,126.2,126.2,126.1,122.7$, $122.1,121.4,119.9,118.8,114.3,114.1,113.4,100.2,84.6,41.6,41.6,41.2,36.1,31.3,28.9,28.2$, 27.5. IR $(\mathrm{KBr}) v 3453,2953,1610,1569,1498,1261,1034,755 \mathrm{~cm}^{-1}$. HRMS (ESI) calcd for $\mathrm{C}_{44} \mathrm{H}_{40} \mathrm{~N}_{3} \mathrm{O}[\mathrm{M}+\mathrm{H}]^{+}$626.3166, found 626.3159.

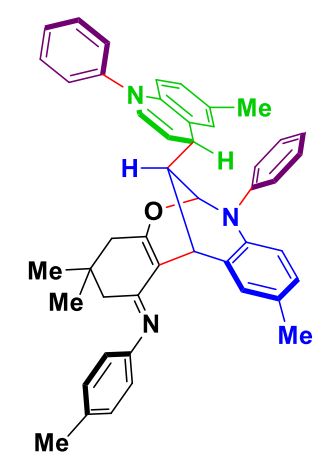

3,3,10-trimethyl-13-(6-methyl-1-phenyl-1,4-dihydroquinolin-4-yl)-7-phenyl- $N$-( $p$-tolyl)-2,3,4,6,7, 12-hexahydro-1H-6,12-methanodibenzo[d,g][1,3]oxazocin-1-imine (6e) White solid obtained by filtration of the precipitate; $70.3 \mathrm{mg}, 70 \%$ yield; $\mathrm{dr}>20: 1$; reaction time $=$ $6 \mathrm{~h} ; \mathrm{mp} 223.9-224.6{ }^{\circ} \mathrm{C} ;{ }^{1} \mathrm{H}$ NMR $\left(400 \mathrm{MHz} \mathrm{CDCl}_{3}\right) \delta$ 7.54-7.39 (m, 7H), 7.33-7.22 (m, 4H), $7.09(\mathrm{~d}, J=8.0 \mathrm{~Hz}, 2 \mathrm{H}), 6.95(\mathrm{~s}, 1 \mathrm{H}), 6.79(\mathrm{~d}, J=8.0 \mathrm{~Hz}, 2 \mathrm{H}), 6.67-6.54(\mathrm{~m}, 5 \mathrm{H}), 5.43(\mathrm{~s}, 1 \mathrm{H})$, 4.99-4.95 (m, 1H), $4.62(\mathrm{~s}, 1 \mathrm{H}), 3.21-3.18(\mathrm{~m}, 1 \mathrm{H}), 2.32(\mathrm{~s}, 3 \mathrm{H}), 2.27(\mathrm{~s}, 3 \mathrm{H}), 2.25-2.22(\mathrm{~m}, 1 \mathrm{H})$, 
$2.19(\mathrm{~s}, 3 \mathrm{H}), 2.12(\mathrm{~d}, J=16.0 \mathrm{~Hz}, 1 \mathrm{H}), 2.03(\mathrm{~s}, 2 \mathrm{H}), 1.86(\mathrm{~d}, J=16.0 \mathrm{~Hz}, 1 \mathrm{H}), 0.92(\mathrm{~s}, 3 \mathrm{H}), 0.80$ (s, 3H); ${ }^{13} \mathrm{C}$ NMR (100 MHz, $\left.\mathrm{CDCl}_{3}\right) \delta 163.2,158.3,150.1,145.4,143.9,139.5,139.4,131.9$, $131.2,130.5,130.4,129.9,129.7,129.5,129.3,127.9,127.2,126.6,126.3,126.2,125.9,125.7$, 122.6, 119.7, 114.4, 113.9, 113.2, 99.9, 84.9, 41.6, 41.6, 41.1, 36.2, 31.3, 29.0, 28.1, 27.5, 20.8, 20.5, 20.3. IR $(\mathrm{KBr}) v 3442,3253,1610,1499,1271,1033,760 \mathrm{~cm}^{-1}$. HRMS (ESI) calcd for $\mathrm{C}_{47} \mathrm{H}_{46} \mathrm{~N}_{3} \mathrm{O}[\mathrm{M}+\mathrm{H}]^{+}$668.3635, found 668.3661 .

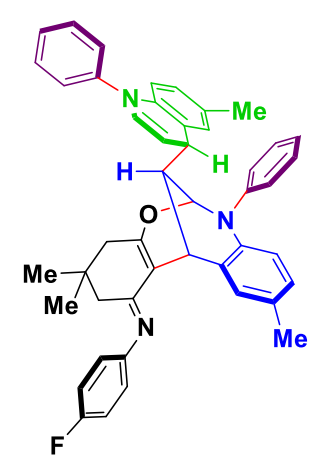

$N$-(4-fluorophenyl)-3,3,10-trimethyl-13-(6-methyl-1-phenyl-1,4-dihydroquinolin-4-yl)-7-phenyl-2, 3,4,6,7,12-hexahydro-1H-6,12-methanodibenzo[d,g][1,3] oxazocin-1-imine (6f)

White solid obtained by filtration of the precipitate; $63.4 \mathrm{mg}$, 63\% yield; $\mathrm{dr}>20: 1$; reaction time $=$ $6 \mathrm{~h} ; \mathrm{mp} 233.4-233.9^{\circ} \mathrm{C} ;{ }^{1} \mathrm{H}$ NMR $\left(400 \mathrm{MHz}, \mathrm{CDCl}_{3}\right) \delta 7.42\left(\mathrm{dd}, J_{I}=J_{2}=4.0 \mathrm{~Hz}, 7 \mathrm{H}\right), 7.29-7.22$ (m, 4H), 7.00-6.94 (m, 3H), $6.81(\mathrm{t}, J=4.0 \mathrm{~Hz}, 1 \mathrm{H}), 6.79(\mathrm{t}, J=4.0 \mathrm{~Hz}, 1 \mathrm{H}), 6.67-6.60(\mathrm{~m}, 4 \mathrm{H})$, $6.56(\mathrm{~d}, J=8.0 \mathrm{~Hz}, 1 \mathrm{H}), 5.44(\mathrm{t}, J=4.0 \mathrm{~Hz}, 1 \mathrm{H}), 4.97(\mathrm{t}, J=8.0 \mathrm{~Hz}, 1 \mathrm{H}), 4.59(\mathrm{~s}, 1 \mathrm{H}), 3.20(\mathrm{q}, J=$ $8.0 \mathrm{~Hz}, 1 \mathrm{H}), 2.27(\mathrm{~s}, 3 \mathrm{H}), 2.22\left(\mathrm{tt}, J_{l}=J_{2}=4.0 \mathrm{~Hz}, 1 \mathrm{H}\right), 2.19(\mathrm{~s}, 3 \mathrm{H}), 2.09(\mathrm{~d}, J=16.0 \mathrm{~Hz}, 1 \mathrm{H})$, $2.04(\mathrm{~s}, 2 \mathrm{H}), 1.84(\mathrm{~d}, J=16.0 \mathrm{~Hz}, 1 \mathrm{H}), 0.92(\mathrm{~s}, 3 \mathrm{H}), 0.81(\mathrm{~s}, 3 \mathrm{H}) ;{ }^{13} \mathrm{C} \mathrm{NMR}\left(100 \mathrm{MHz}, \mathrm{CDCl}_{3}\right) \delta$ 164.2, 158.8, $158.8(\mathrm{~d}, J=238.0 \mathrm{~Hz}, 1 \mathrm{C}), 148.8(\mathrm{~d}, J=3.0 \mathrm{~Hz}, 1 \mathrm{C}), 145.3,143.9,139.4(\mathrm{~d}, J=$ $16.0 \mathrm{~Hz}, 1 \mathrm{C}), 131.9,130.6,130.3,129.9,129.7,129.6,127.9,127.2,126.7,126.3,126.0,125.9$, 125.8, 122.6, 120.9 (d, $J=8.0 \mathrm{~Hz}, 1 \mathrm{C}), 115.5,115.3,114.4,114.0,113.3,99.9,85.0,41.6,41.5$, 41.2, 36.2, 31.3, 29.0, 28.1, 27.5, 20.5, 20.3. IR (KBr) v 3439, 2956, 1607, 1497, 1212, $760 \mathrm{~cm}^{-1}$. HRMS (ESI) calcd for $\mathrm{C}_{46} \mathrm{H}_{43} \mathrm{FN}_{3} \mathrm{O}[\mathrm{M}+\mathrm{H}]^{+} 672.3385$, found 672.3392 . 


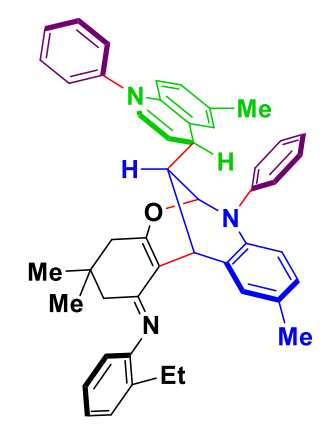

$N$-(2-ethylphenyl)-3,3,10-trimethyl-13-(6-methyl-1-phenyl-1,4-dihydroquinolin-4-yl)-7-phenyl-2, 3,4,6,7,12-hexahydro-1H-6,12-methanodibenzo[d,g][1,3]oxazocin-1-imine $(\mathbf{6 g})$

White solid obtained by filtration of the precipitate; $69.8 \mathrm{mg}, 68 \%$ yield; $\mathrm{dr}>20: 1$; reaction time $=$ 6 h; mp 239.4-239.6 ${ }^{\circ} \mathrm{C} ;{ }^{1} \mathrm{H}$ NMR $\left(300 \mathrm{MHz}, \mathrm{CDCl}_{3}\right) \delta 7.21(\mathrm{~d}, J=3.0 \mathrm{~Hz}, 6 \mathrm{H}), 7.08-6.99(\mathrm{~m}$, $6 \mathrm{H}), 6.87(\mathrm{t}, J=6.0 \mathrm{~Hz}, 1 \mathrm{H}), 6.75(\mathrm{t}, J=6.0 \mathrm{~Hz}, 2 \mathrm{H}), 6.57(\mathrm{~d}, J=6.0 \mathrm{~Hz}, 2 \mathrm{H}), 6.43(\mathrm{~d}, J=6.0 \mathrm{~Hz}$, 1H), 6.38-6.29 (m, 3H), $5.19(\mathrm{~s}, 1 \mathrm{H}), 4.74(\mathrm{t}, J=3.0 \mathrm{~Hz}, 1 \mathrm{H}), 4.40(\mathrm{~s}, 1 \mathrm{H}), 2.96-2.93(\mathrm{~m}, 1 \mathrm{H})$, 2.27-2.24 (m, 2H), $1.99(\mathrm{~d}, J=15.0 \mathrm{~Hz}, 7 \mathrm{H}), 1.84(\mathrm{~d}, J=18.0 \mathrm{~Hz}, 3 \mathrm{H}), 1.58(\mathrm{~d}, J=18.0 \mathrm{~Hz}, 1 \mathrm{H})$, $0.87(\mathrm{t}, J=6.0 \mathrm{~Hz}, 3 \mathrm{H}), 0.68(\mathrm{~s}, 3 \mathrm{H}), 0.55(\mathrm{~s}, 3 \mathrm{H}) ;{ }^{13} \mathrm{C} \mathrm{NMR}\left(75 \mathrm{MHz}, \mathrm{CDCl}_{3}\right) \delta 163.3,158.6$, $150.9,145.4,143.9,139.5,139.3,133.7,131.8,130.6,130.5,130.0,129.8,129.7,129.6,129.5$, $128.0,127.9,127.2,126.8,126.3,126.0,125.8,125.7,122.7,122.4,119.3,114.5,114.0,113.3$, $100.0,84.8,41.8,41.7,36.2,31.4,28.4,28.2,24.2,20.3,14.0$, three carbons missing in the aliphatic region. IR $(\mathrm{KBr}) v 3441,2958,1604,1496,1270,763 \mathrm{~cm}^{-1}$. HRMS (ESI) calcd for $\mathrm{C}_{48} \mathrm{H}_{48} \mathrm{~N}_{3} \mathrm{O}[\mathrm{M}+\mathrm{H}]^{+}$682.3792, found 682.3811.

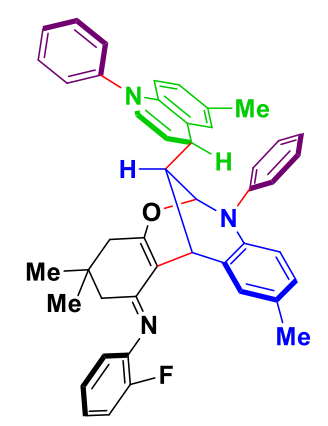

$N$-(2-fluorophenyl)-3,3,10-trimethyl-13-(6-methyl-1-phenyl-1,4-dihydroquinolin-4-yl)-7-phenyl-2 ,3,4,6,7,12-hexahydro-1H-6,12-methanodibenzo[d,g][1,3]oxazocin-1-imine (6h)

White solid obtained by filtration of the precipitate; $66.2 \mathrm{mg}, 66 \%$ yield; $\mathrm{dr}>20: 1$; reaction time $=$ $6 \mathrm{~h} ; \mathrm{mp} 221.2-221.3{ }^{\circ} \mathrm{C} ;{ }^{1} \mathrm{H}$ NMR $\left(300 \mathrm{MHz} \mathrm{CDCl}_{3}\right) \delta$ 7.48-7.41 (m, 7H), 7.31-7.23 (m, 4H), 7.09-6.94 (m, 4H), 6.81-6.74 (m, 3H), 6.67-6.54 (m, 3H), $5.43(\mathrm{~s}, 1 \mathrm{H}), 4.97(\mathrm{t}, J=6.0 \mathrm{~Hz}, 1 \mathrm{H})$, $4.62(\mathrm{~s}, 1 \mathrm{H}), 3.21(\mathrm{t}, J=6.0 \mathrm{~Hz}, 1 \mathrm{H}), 2.28-2.10(\mathrm{~m}, 7 \mathrm{H}), 1.86(\mathrm{~d}, J=9.0 \mathrm{~Hz}, 1 \mathrm{H}), 2.06(\mathrm{~s}, 3 \mathrm{H})$, 
$0.94(\mathrm{~s}, 3 \mathrm{H}), 0.81(\mathrm{~s}, 3 \mathrm{H}) ;{ }^{13} \mathrm{C} \mathrm{NMR}\left(75 \mathrm{MHz}, \mathrm{CDCl}_{3}\right) \delta 165.9,159.6,152.6(\mathrm{~d}, J=241.5 \mathrm{~Hz}, 1 \mathrm{C})$, 145.4, 143.9, 140.1 (d, $J=10.5 \mathrm{~Hz}, 1 \mathrm{C}), 139.4$ (d, $J=28.5 \mathrm{~Hz}, 1 \mathrm{C}), 132.0,130.6,129.9,129.7$, 129.6, 128.1, 127.2, 126.7, 126.4, 125.9, 125.8, 124.1, 123.2, 123.1, 123.1, 122.6, 122.6, 122.6, 115.9, 115.6, 114.6, 113.9, 113.2, 99.8, 85.2, 41.7, 41.5, 41.4, 36.2, 31.4, 28.8, 28.0, 27.6, 20.4, 20.3. IR $(\mathrm{KBr}) \vee 3444,2952,1623,1495,1265,1033,759 \mathrm{~cm}^{-1}$. HRMS (ESI) calcd for $\mathrm{C}_{46} \mathrm{H}_{43} \mathrm{FN}_{3} \mathrm{O}[\mathrm{M}+\mathrm{H}]^{+} 672.3385$, found 672.3362 .

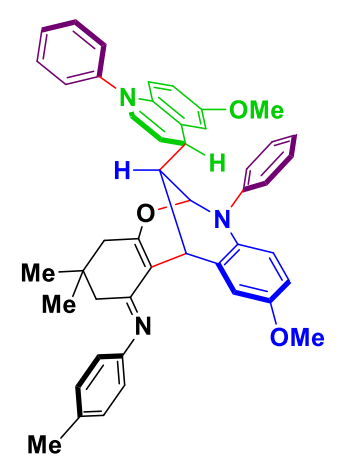

10-methoxy-13-(6-methoxy-1-phenyl-1,4-dihydroquinolin-4-yl)-3,3-dimethyl-7-phenyl- $N$-( $p$-tolyl) -2,3,4,6,7,12-hexahydro-1H-6,12-methanodibenzo[ $d, g][1,3]$ oxazocin-1-imine (6i)

White solid obtained by filtration of the precipitate; $61.2 \mathrm{mg}, 58 \%$ yield; $\mathrm{dr}>20: 1$; reaction time $=$ $6 \mathrm{~h} ; \mathrm{mp} 218.1-218.7^{\circ} \mathrm{C} ;{ }^{1} \mathrm{H}$ NMR $\left(400 \mathrm{MHz}, \mathrm{CDCl}_{3}\right) \delta 7.33(\mathrm{t}, J=8.0 \mathrm{~Hz}, 6 \mathrm{H}), 7.23-7.15(\mathrm{~m}, 5 \mathrm{H})$, $7.01(\mathrm{~d}, J=8.0 \mathrm{~Hz}, 2 \mathrm{H}), 6.63-6.49(\mathrm{~m}, 8 \mathrm{H}), 5.44(\mathrm{~s}, 1 \mathrm{H}), 4.84(\mathrm{t}, J=8.0 \mathrm{~Hz}, 1 \mathrm{H}), 4.55(\mathrm{~s}, 1 \mathrm{H})$, $3.67(\mathrm{~s}, 3 \mathrm{H}), 3.46(\mathrm{~s}, 3 \mathrm{H}), 3.16(\mathrm{q}, J=8.0 \mathrm{~Hz}, 1 \mathrm{H}), 2.24(\mathrm{~s}, 3 \mathrm{H}), 2.17$ (d, $J=8.0 \mathrm{~Hz}, 1 \mathrm{H}), 2.02(\mathrm{~d}, J$ $=16.0 \mathrm{~Hz}, 1 \mathrm{H}), 1.97(\mathrm{~s}, 2 \mathrm{H}), 1.81(\mathrm{~d}, J=16.0 \mathrm{~Hz}, 1 \mathrm{H}), 0.84(\mathrm{~s}, 3 \mathrm{H}), 0.73(\mathrm{~s}, 3 \mathrm{H}) ;{ }^{13} \mathrm{C}$ NMR $(100$ $\left.\mathrm{MHz}, \mathrm{CDCl}_{3}\right) \delta 163.4,158.7,154.8,152.6,150.2,145.6,144.0,135.8,135.4,132.0,131.3,129.7$, $129.7,129.4,127.3,127.0,126.0,125.7,125.6,124.1,119.7,115.3,114.3,114.2,113.8,113.1$, 112.8, 99.1, 85.1, 55.7, 55.6, 41.6, 41.6, 41.2, 36.7, 31.3, 28.8, 28.4, 27.7, 20.8. IR (KBr) v 3446, 2948, 1606, 1496, 1219, $760 \mathrm{~cm}^{-1}$. HRMS (ESI) calcd for $\mathrm{C}_{47} \mathrm{H}_{46} \mathrm{~N}_{3} \mathrm{O}_{3}[\mathrm{M}+\mathrm{H}]^{+} 700.3534$, found 700.3512 .

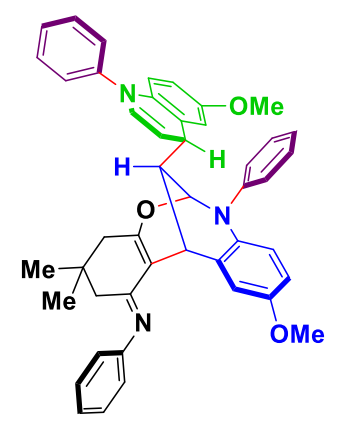


10-methoxy-13-(6-methoxy-1-phenyl-1,4-dihydroquinolin-4-yl)-3,3-dimethyl- $N, 7$-diphenyl-2,3,4, 6,7,12-hexahydro-1H-6,12-methanodibenzo[d,g][1,3]oxazocin-1-imine $(\mathbf{6 j})$

White solid obtained by filtration of the precipitate; $62.9 \mathrm{mg}, 62 \%$ yield; $\mathrm{dr}>20: 1$; reaction time $=$ 6 h; mp 196.3-196.9 ${ }^{\circ} \mathrm{C} ;{ }^{1} \mathrm{H}$ NMR (400 MHz, $\left.\mathrm{CDCl}_{3}\right) \delta$ 7.47-7.37 (m, 10H), $7.28(\mathrm{t}, J=8.0$ Hz,4H), $6.93(\mathrm{~s}, 1 \mathrm{H}), 6.80(\mathrm{~d}, J=8.0 \mathrm{~Hz}, 2 \mathrm{H}), 6.64(\mathrm{~d}, J=8.0 \mathrm{~Hz}, 1 \mathrm{H}), 6.61(\mathrm{~d}, J=8.0 \mathrm{~Hz}, 1 \mathrm{H})$, $6.56\left(\mathrm{dd}, J_{l}=J_{2}=4.0 \mathrm{~Hz}, 3 \mathrm{H}\right), 5.43(\mathrm{t}, J=4.0 \mathrm{~Hz}, 1 \mathrm{H}), 4.96(\mathrm{t}, J=8.0 \mathrm{~Hz}, 1 \mathrm{H}), 4.55(\mathrm{~s}, 1 \mathrm{H}), 3.18$ (q, $J=8.0 \mathrm{~Hz}, 1 \mathrm{H}), 2.26(\mathrm{~s}, 3 \mathrm{H}), 2.22(\mathrm{t}, J=4.0 \mathrm{~Hz}, 1 \mathrm{H}), 2.20(\mathrm{~s}, 3 \mathrm{H}), 2.06(\mathrm{~d}, J=16.0 \mathrm{~Hz}, 1 \mathrm{H})$, $2.04(\mathrm{~s}, 2 \mathrm{H}), 1.84(\mathrm{~d}, J=16.0 \mathrm{~Hz}, 1 \mathrm{H}), 0.92(\mathrm{~s}, 3 \mathrm{H}), 0.81(\mathrm{~s}, 3 \mathrm{H}) ;{ }^{13} \mathrm{C} \mathrm{NMR}\left(100 \mathrm{MHz}, \mathrm{CDCl}_{3}\right) \delta$ 163.9, 159.2, 151.8, 145.3, 143.9, 139.5, 139.4, 131.9, 131.7, 130.7, 130.3, 129.9, 129.7, 129.6, 128.0, 127.2, 126.8, 126.3, 126.0, 125.9, 125.8, 122.6, 121.7, 114.8, 114.3, 114.0, 113.3, 99.9, 85.0, 41.6, 41.5, 41.3, 36.1, 31.4, 29.0, 28.1, 27.5, 20.5, 20.3. IR (KBr) v 3443, 2954, 1607, 1493, 1379, 1219, $761 \mathrm{~cm}^{-1}$. HRMS (ESI) calcd for $\mathrm{C}_{46} \mathrm{H}_{44} \mathrm{~N}_{3} \mathrm{O}_{3}[\mathrm{M}+\mathrm{H}]^{+} 686.3377$, found 686.3351 .

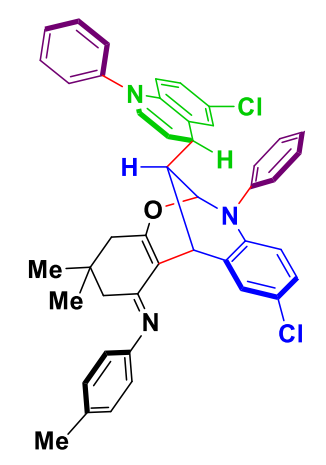

10-chloro-13-(6-chloro-1-phenyl-1,4-dihydroquinolin-4-yl)-3,3-dimethyl-7-phenyl- $N$-( $p$-tolyl)-2,3, 4,6,7,12-hexahydro-1H-6,12-methanodibenzo[ $[$, $g][1,3]$ oxazocin-1-imine $(\mathbf{6 k})$

White solid obtained by filtration of the precipitate; $67.2 \mathrm{mg}, 63 \%$ yield; $\mathrm{dr}>20: 1$; reaction time $=$ $6 \mathrm{~h} ; \mathrm{mp} 253.2-253.7{ }^{\circ} \mathrm{C} ;{ }^{1} \mathrm{H}$ NMR $\left(400 \mathrm{MHz}, \mathrm{CDCl}_{3}\right) \delta 7.62(\mathrm{~d}, J=4.0 \mathrm{~Hz}, 1 \mathrm{H}), 7.47(\mathrm{q}, J=8.0$ $\mathrm{Hz}, 4 \mathrm{H}), 7.38(\mathrm{~d}, J=4.0 \mathrm{~Hz}, 2 \mathrm{H}), 7.34-7.27(\mathrm{~m}, 4 \mathrm{H}), 7.17(\mathrm{~d}, J=4.0 \mathrm{~Hz}, 1 \mathrm{H}), 7.11(\mathrm{~d}, J=8.0 \mathrm{~Hz}$, $2 \mathrm{H}), 6.97\left(\mathrm{dd}, J_{l}=J_{2}=4.0 \mathrm{~Hz}, 1 \mathrm{H}\right), 6.93\left(\mathrm{dd}, J_{l}=J_{2}=4.0 \mathrm{~Hz}, 1 \mathrm{H}\right), 6.62-6.54(\mathrm{~m}, 5 \mathrm{H}), 5.37(\mathrm{~s}$, $1 \mathrm{H}), 4.96(\mathrm{t}, J=8.0 \mathrm{~Hz}, 1 \mathrm{H}), 4.58(\mathrm{~s}, 1 \mathrm{H}), 3.15(\mathrm{q}, J=8.0 \mathrm{~Hz}, 1 \mathrm{H}), 2.33(\mathrm{~s}, 3 \mathrm{H}), 2.26\left(\mathrm{tt}, J_{1}=J_{2}=\right.$ $4.0 \mathrm{~Hz}, 1 \mathrm{H}), 2.14$ (d, $J=16.0 \mathrm{~Hz}, 1 \mathrm{H}), 2.04(\mathrm{~s}, 2 \mathrm{H}), 1.90$ (d, $J=16.0 \mathrm{~Hz}, 1 \mathrm{H}), 0.93(\mathrm{~s}, 3 \mathrm{H}), 0.81$ (s, 3H); ${ }^{13} \mathrm{C}$ NMR (100 MHz, $\left.\mathrm{CDCl}_{3}\right) \delta 163.0,158.3,149.7,144.6,143.3,140.9,140.6,132.2$, 131.6, 130.0, 129.4, 129.3, 128.9, 127.7, 126.7, 126.7, 126.6, 126.6, 126.2, 126.0, 125.0, 123.7, 123.6, 119.7, 115.3, 114.8, 113.8, 99.6, 84.1, 41.5, 41.4, 41.0, 36.1, 31.3, 28.7, 28.1, 27.7, 20.8. IR 
$(\mathrm{KBr}) v 3441,2955,1610,1490,1266,1173,1033,758 \mathrm{~cm}^{-1}$. HRMS (ESI) calcd for $\mathrm{C}_{45} \mathrm{H}_{40} \mathrm{Cl}_{2} \mathrm{~N}_{3} \mathrm{O}[\mathrm{M}+\mathrm{H}]^{+}$708.2543, found 708.2531.

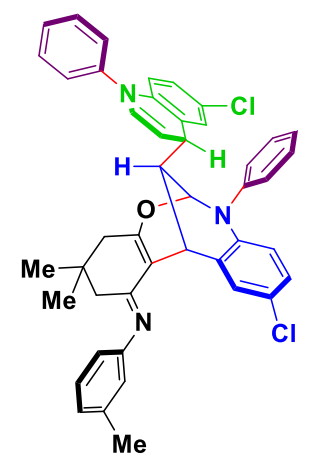

10-chloro-13-(6-chloro-1-phenyl-1,4-dihydroquinolin-4-yl)-3,3-dimethyl-7-phenyl- $N$-( $m$-tolyl)-2, 3,4,6,7,12-hexahydro-1H-6,12-methanodibenzo[ $[$, $g][1,3]$ oxazocin-1-imine (6l)

White solid obtained by filtration of the precipitate; $63.1 \mathrm{mg}, 59 \%$ yield; $\mathrm{dr}>20: 1$; reaction time $=$ $6 \mathrm{~h}$; mp 247.0-247.6 ${ }^{\circ} \mathrm{C} ;{ }^{1} \mathrm{H}$ NMR $\left(400 \mathrm{MHz}, \mathrm{CDCl}_{3}\right) \delta 7.54(\mathrm{~d}, J=4.0 \mathrm{~Hz}, 1 \mathrm{H}), 7.42-7.35(\mathrm{~m}$, 4H), $7.31(\mathrm{~d}, J=8.0 \mathrm{~Hz}, 2 \mathrm{H}), 7.24-7.19(\mathrm{~m}, 4 \mathrm{H}), 7.12-7.09(\mathrm{~m}, 2 \mathrm{H}), 6.90-6.84(\mathrm{~m}, 2 \mathrm{H}), 6.76(\mathrm{~d}, J$ $=8.0 \mathrm{~Hz}, 1 \mathrm{H}), 6.52-6.46(\mathrm{~m}, 4 \mathrm{H}), 6.42(\mathrm{~d}, J=8.0 \mathrm{~Hz}, 1 \mathrm{H}), 5.30(\mathrm{t}, J=4.0 \mathrm{~Hz}, 1 \mathrm{H}), 4.87(\mathrm{t}, J=8.0$ $\mathrm{Hz}, 1 \mathrm{H}), 4.50(\mathrm{~s}, 1 \mathrm{H}), 3.09(\mathrm{q}, J=8.0 \mathrm{~Hz}, 1 \mathrm{H}), 2.25(\mathrm{~s}, 3 \mathrm{H}), 2.18\left(\mathrm{tt}, J_{I}=J_{2}=4.0 \mathrm{~Hz}, 1 \mathrm{H}\right), 2.06(\mathrm{~d}$, $J=16.0 \mathrm{~Hz}, 1 \mathrm{H}), 1.96(\mathrm{~s}, 2 \mathrm{H}), 1.82(\mathrm{~d}, J=16.0 \mathrm{~Hz}, 1 \mathrm{H}), 0.86(\mathrm{~s}, 3 \mathrm{H}), 0.74(\mathrm{~s}, 3 \mathrm{H}) ;{ }^{13} \mathrm{C} \mathrm{NMR}$ $\left(100 \mathrm{MHz}, \mathrm{CDCl}_{3}\right) \delta 162.8,158.4,152.3,144.5,143.3,140.8,140.6,138.6,132.2,130.0,129.3$, $128.9,128.7,127.6,126.7,126.6,126.6,126.2,126.0,123.7,123.6,123.1,120.5,116.7,115.3$, $114.8,113.7,99.6,84.2,41.5,41.3,41.1,36.1,31.3,28.7,28.1,27.7,21.5$, two carbons missing in the aromatic region. IR (KBr) $v 3443,2956,1622,1485,1378,753 \mathrm{~cm}^{-1}$. HRMS (ESI) calcd for $\mathrm{C}_{45} \mathrm{H}_{39} \mathrm{Cl}_{2} \mathrm{~N}_{3} \mathrm{NaO}[\mathrm{M}+\mathrm{Na}]^{+} 730.2362$, found 730.2349 .

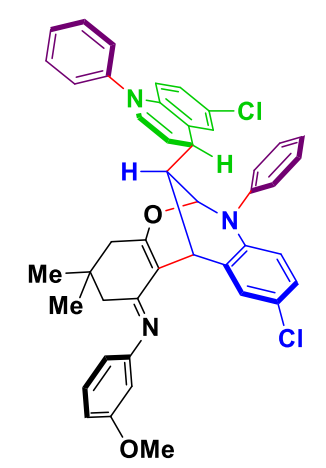

10-chloro-13-(6-chloro-1-phenyl-1,4-dihydroquinolin-4-yl)- $N$-(3-methoxyphenyl)-3,3-dimethyl-7phenyl-2,3,4,6,7,12-hexahydro-1H-6,12-methanodibenzo[d,g][1,3] oxazocin-1-imine (6m) 
White solid obtained by filtration of the precipitate; $68.9 \mathrm{mg}, 63 \%$ yield; $\mathrm{dr}>20: 1$; reaction time $=$ $6 \mathrm{~h} ; \mathrm{mp} 249.9-250.8{ }^{\circ} \mathrm{C} ;{ }^{1} \mathrm{H}$ NMR $\left(400 \mathrm{MHz}, \mathrm{CDCl}_{3}\right) \delta 7.56(\mathrm{~d}, J=4.0 \mathrm{~Hz}, 1 \mathrm{H}), 7.42-7.36(\mathrm{~m}$, 4H), $7.31(\mathrm{~d}, J=8.0 \mathrm{~Hz}, 2 \mathrm{H}), 7.24-7.19(\mathrm{~m}, 4 \mathrm{H}), 7.14-7.09$ (m, 2H), 6.90-6.84 (m, 2H), 6.52-6.46 $(\mathrm{m}, 4 \mathrm{H}), 6.21(\mathrm{~d}, J=8.0 \mathrm{~Hz}, 2 \mathrm{H}), 5.30(\mathrm{t}, J=4.0 \mathrm{~Hz}, 1 \mathrm{H}), 4.87(\mathrm{t}, J=8.0 \mathrm{~Hz}, 1 \mathrm{H}), 4.49$ (s, 1H), $3.72(\mathrm{~s}, 3 \mathrm{H}), 3.09(\mathrm{q}, J=8.0 \mathrm{~Hz}, 1 \mathrm{H}), 2.19\left(\mathrm{tt}, J_{l}=J_{2}=4.0 \mathrm{~Hz}, 1 \mathrm{H}\right), 2.08(\mathrm{~d}, J=16.0 \mathrm{~Hz}, 1 \mathrm{H})$, $1.97(\mathrm{~s}, 2 \mathrm{H}), 1.83(\mathrm{~d}, J=16.0 \mathrm{~Hz}, 1 \mathrm{H}), 0.87$ (s, 3H), $0.75(\mathrm{~s}, 3 \mathrm{H}) ;{ }^{13} \mathrm{C} \mathrm{NMR}\left(100 \mathrm{MHz}, \mathrm{CDCl}_{3}\right) \delta$ 163.0, 160.3, 158.7, 153.8, 144.5, 143.3, 140.8, 140.5, 132.2, 130.0, 129.6, 129.3, 128.8, 127.6, 127.6, 126.7, 126.6, 126.2, 126.0, 123.7, 123.6, 115.3, 114.8, 113.7, 112.1, 108.4, 105.1, 99.6, 84.2, 55.2, 41.5, 41.3, 41.0, 36.1, 31.3, 28.7, 28.1, 27.7, two carbons missing in the aromatic region. IR $(\mathrm{KBr}) v 3441,2954,1593,1485,1379,1289,1146,768 \mathrm{~cm}^{-1}$. HRMS (ESI) calcd for $\mathrm{C}_{45} \mathrm{H}_{40} \mathrm{Cl}_{2} \mathrm{~N}_{3} \mathrm{O}_{2}[\mathrm{M}+\mathrm{H}]^{+} 724.2492$, found 724.2484 .

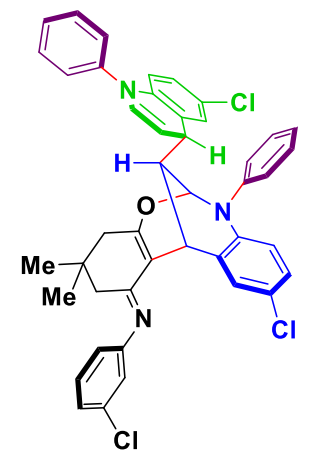

10-chloro-13-(6-chloro-1-phenyl-1,4-dihydroquinolin-4-yl)- $N$-(3-chlorophenyl)-3,3-dimethyl-7-ph enyl-2,3,4,6,7,12-hexahydro-1 $H$-6,12-methanodibenzo[ $[d, g][1,3]$ oxazocin-1-imine (6n)

White solid obtained by filtration of the precipitate; $66.0 \mathrm{mg}, 60 \%$ yield; $\mathrm{dr}>20: 1$; reaction time $=$ $6 \mathrm{~h}$; mp 244.7-245.5 ${ }^{\circ} \mathrm{C} ;{ }^{1} \mathrm{H}$ NMR $\left(400 \mathrm{MHz}, \mathrm{CDCl}_{3}\right) \delta 7.57(\mathrm{~d}, J=4.0 \mathrm{~Hz}, 1 \mathrm{H}), 7.50-7.44(\mathrm{~m}$, 4H), $7.39(\mathrm{~d}, J=8.0 \mathrm{~Hz}, 2 \mathrm{H}), 7.34-7.27(\mathrm{~m}, 4 \mathrm{H}), 7.21(\mathrm{t}, J=8.0 \mathrm{~Hz}, 1 \mathrm{H}), 7.16(\mathrm{~d}, J=4.0 \mathrm{~Hz}, 1 \mathrm{H})$, 7.00-6.93 (m, 3H), $6.72(\mathrm{t}, J=4.0 \mathrm{~Hz}, 1 \mathrm{H}), 6.60-6.54(\mathrm{~m}, 4 \mathrm{H}), 5.39(\mathrm{t}, J=4.0 \mathrm{~Hz}, 1 \mathrm{H}), 4.94(\mathrm{t}, J=$ $8.0 \mathrm{~Hz}, 1 \mathrm{H}), 4.53(\mathrm{~s}, 1 \mathrm{H}), 3.15(\mathrm{q}, J=8.0 \mathrm{~Hz}, 1 \mathrm{H}), 2.25\left(\mathrm{tt}, J_{l}=J_{2}=4.0 \mathrm{~Hz}, 1 \mathrm{H}\right), 2.10(\mathrm{~d}, J=16.0$ $\mathrm{Hz}, 1 \mathrm{H}), 2.06$ (s, 2H), 1.91 (d, $J=16.0 \mathrm{~Hz}, 1 \mathrm{H}), 0.94$ (s, 3H), 0.83 (s, 3H); ${ }^{13} \mathrm{C}$ NMR (100 MHz, $\left.\mathrm{CDCl}_{3}\right) \delta 163.8,159.4,153.7,144.4,143.3,140.8,140.6,134.4,132.3,130.0,130.0,129.9,129.2$, 128.8, 127.6, 127.4, 126.7, 126.7, 126.6, 126.3, 126.0, 123.7, 123.6, 122.3, 119.9, 118.2, 115.3, $114.9,113.6,99.4,84.3,41.5,41.3,36.1,31.4,28.6,28.1,27.7$, one carbon missing in the aliphatic region. IR $(\mathrm{KBr}) v 3438,2956,1618,1588,1486,754 \mathrm{~cm}^{-1}$. HRMS (ESI) calcd for $\mathrm{C}_{44} \mathrm{H}_{36} \mathrm{Cl}_{3} \mathrm{~N}_{3} \mathrm{NaO}[\mathrm{M}+\mathrm{Na}]^{+} 750.1816$, found 750.1795 . 


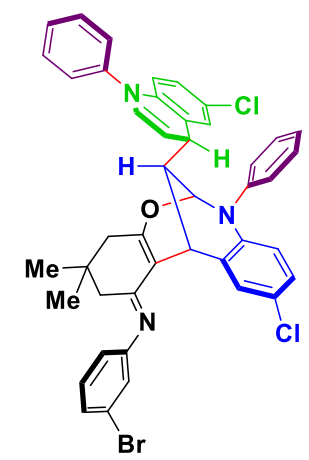

$N$-(3-bromophenyl)-10-chloro-13-(6-chloro-1-phenyl-1,4-dihydroquinolin-4-yl)-3,3-dimethyl-7-p henyl-2,3,4,6,7,12-hexahydro-1 $H$-6,12-methanodibenzo[ $d, g][1,3]$ oxazocin-1-imine (6o)

White solid obtained by filtration of the precipitate; $66.8 \mathrm{mg}, 58 \%$ yield; $\mathrm{dr}>20: 1$; reaction time $=$ $6 \mathrm{~h} ; \mathrm{mp} 241.8-242.5{ }^{\circ} \mathrm{C} ;{ }^{1} \mathrm{H}$ NMR $\left(400 \mathrm{MHz}, \mathrm{CDCl}_{3}\right) \delta 7.56(\mathrm{~d}, J=4.0 \mathrm{~Hz}, 1 \mathrm{H}), 7.48(\mathrm{q}, J=8.0$ Hz, 4H), 7.39-7.27 (m, 6H), 7.15 (d, $J=4.0 \mathrm{~Hz}, 3 \mathrm{H}), 6.99-6.93$ (m, 2H), 6.88 (s, 1H), 6.63-6.54 (m, 4H), $5.39(\mathrm{~s}, 1 \mathrm{H}), 4.94(\mathrm{t}, J=8.0 \mathrm{~Hz}, 1 \mathrm{H}), 4.52(\mathrm{~s}, 1 \mathrm{H}), 3.15(\mathrm{q}, J=8.0 \mathrm{~Hz}, 1 \mathrm{H}), 2.25(\mathrm{~d}, J=$ $8.0 \mathrm{~Hz}, 1 \mathrm{H}), 2.10(\mathrm{~d}, J=16.0 \mathrm{~Hz}, 1 \mathrm{H}), 2.06(\mathrm{~s}, 2 \mathrm{H}), 1.91(\mathrm{~d}, J=16.0 \mathrm{~Hz}, 1 \mathrm{H}), 0.95(\mathrm{~s}, 3 \mathrm{H}), 0.84$ (s, 3H); ${ }^{13} \mathrm{C}$ NMR $\left(100 \mathrm{MHz}, \mathrm{CDCl}_{3}\right) \delta 163.9,159.5,153.8,144.4,143.3,140.8,140.5,132.3$, $130.2,130.0,130.0,129.2,128.8,127.6,127.4,126.7,126.7,126.6,126.3,126.0,125.2,123.7$, 123.6, 122.8, 122.6, 118.6, 115.3, 114.9, 113.6, 99.4, 84.3, 41.5, 41.3, 36.1, 31.4, 28.6, 28.1, 27.7, one carbon missing in the aliphatic region. IR $(\mathrm{KBr}) v 3442,2957,1615,1589,1486,1380,754$ $\mathrm{cm}^{-1}$. HRMS (ESI) calcd for $\mathrm{C}_{44} \mathrm{H}_{36} \mathrm{BrCl}_{2} \mathrm{~N}_{3} \mathrm{NaO}[\mathrm{M}+\mathrm{Na}]^{+}$794.1311, found 794.1283.

\section{Experimental data for the formation of 7}
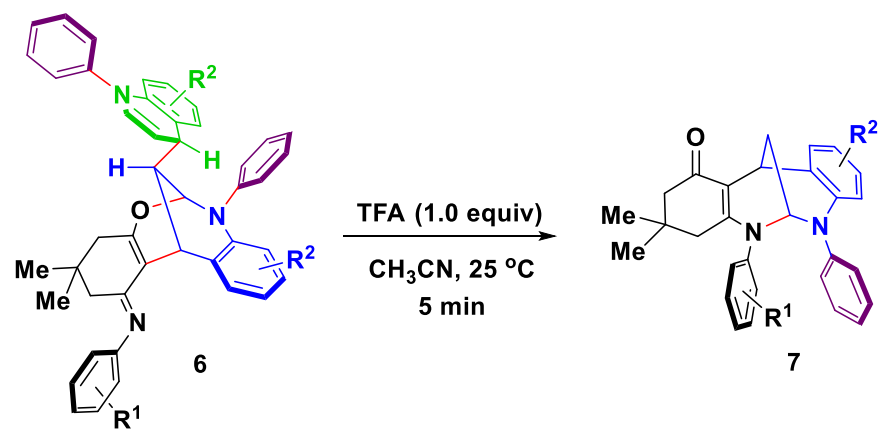

General procedure: To a $5.0 \mathrm{~mL}$ vial were successively added the above-obtained bridged products $6(0.10 \mathrm{mmol})$ with 1.0 equivalent of TFA in $1.0 \mathrm{~mL}$ of $\mathrm{CH}_{3} \mathrm{CN}$. The resulting mixture was stirred at room temperature for $5 \mathrm{~min}$. Upon completion of the reaction (monitoring by TLC), the reaction mixture was directly subjected to flash column chromatography on silica gel (petroleum ether/ ethyl acetate) to afford the corresponding products 7. 


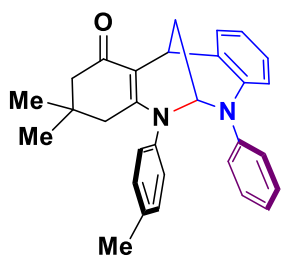

3,3-dimethyl-7-phenyl-5-(p-tolyl)-3,4,5,6,7,12-hexahydro-6,12-methanodibenzo[d,g][1,3]diazocin $-1(2 H)$-one $(7 \mathbf{a})$

Yellow oil obtained by column chromatography (petroleum ether/ethyl acetate $=5: 1$ ); $40.1 \mathrm{mg}, 92 \%$ yield; $\mathrm{dr}>20: 1$; reaction time $=5 \mathrm{~min} ;{ }^{1} \mathrm{H}$ NMR $\left(400 \mathrm{MHz}, \mathrm{CDCl}_{3}\right) \delta 7.43(\mathrm{~d}, J=12.0 \mathrm{~Hz}, 1 \mathrm{H})$, $7.16(\mathrm{t}, J=8.0 \mathrm{~Hz}, 3 \mathrm{H}), 7.03(\mathrm{t}, J=8.0 \mathrm{~Hz}, 3 \mathrm{H}), 6.82(\mathrm{~d}, J=12.0 \mathrm{~Hz}, 1 \mathrm{H}), 6.76(\mathrm{~d}, J=8.0 \mathrm{~Hz}$, 2H), $6.66(\mathrm{t}, J=8.0 \mathrm{~Hz}, 1 \mathrm{H}), 6.52(\mathrm{~d}, J=12.0 \mathrm{~Hz}, 2 \mathrm{H}), 5.21(\mathrm{~s}, 1 \mathrm{H}), 4.42(\mathrm{~s}, 1 \mathrm{H}), 2.28(\mathrm{~s}, 3 \mathrm{H})$, $2.16\left(\mathrm{tt}, J_{I}=J_{2}=4.0 \mathrm{~Hz}, 1 \mathrm{H}\right), 2.10(\mathrm{~s}, 2 \mathrm{H}), 1.94\left(\mathrm{tt}, J_{I}=J_{2}=4.0 \mathrm{~Hz}, 1 \mathrm{H}\right), 1.87(\mathrm{~s}, 2 \mathrm{H}), 0.87(\mathrm{~s}$, 3H), $0.76(\mathrm{~s}, 3 \mathrm{H}) ;{ }^{13} \mathrm{C} \mathrm{NMR}\left(100 \mathrm{MHz}, \mathrm{CDCl}_{3}\right) \delta$ 193.2, 155.6, 147.0, 141.0, 140.8, 137.2, 130.0, $129.8,129.6,128.9,126.8,125.9,125.0,119.2,116.3,113.9,75.8,50.1,41.8,32.8,28.6,27.8$, 26.8, 25.4, 21.0. IR (KBr) $v 3442,2954,1765,1498,1196,757 \mathrm{~cm}^{-1}$. HRMS (ESI) calcd for $\mathrm{C}_{30} \mathrm{H}_{31} \mathrm{~N}_{2} \mathrm{O}[\mathrm{M}+\mathrm{H}]^{+} 435.2431$, found 435.2421.

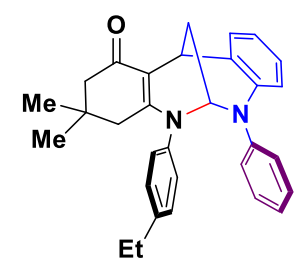

5-(4-ethylphenyl)-3,3-dimethyl-7-phenyl-3,4,5,6,7,12-hexahydro-6,12-methanodibenzo[ $d, g][1,3] \mathrm{d}$ iazocin-1(2H)-one $(\mathbf{7 b})$

Yellow oil obtained by column chromatography (petroleum ether/ethyl acetate $=5: 1$ ); $41.8 \mathrm{mg}, 93 \%$ yield; $\mathrm{dr}>20: 1$; reaction time $=5 \mathrm{~min} ;{ }^{1} \mathrm{H}$ NMR $\left(400 \mathrm{MHz}, \mathrm{CDCl}_{3}\right) \delta 7.51(\mathrm{~d}, J=4.0 \mathrm{~Hz}, 1 \mathrm{H})$, $7.20(\mathrm{t}, J=8.0 \mathrm{~Hz}, 4 \mathrm{H}), 7.09(\mathrm{t}, J=8.0 \mathrm{~Hz}, 1 \mathrm{H}), 6.91(\mathrm{t}, J=8.0 \mathrm{~Hz}, 2 \mathrm{H}), 6.76(\mathrm{t}, J=8.0 \mathrm{~Hz}, 3 \mathrm{H})$, $6.62(\mathrm{~d}, J=8.0 \mathrm{~Hz}, 2 \mathrm{H}), 5.37(\mathrm{~s}, 1 \mathrm{H}), 4.54(\mathrm{~s}, 1 \mathrm{H}), 2.68(\mathrm{q}, J=8.0 \mathrm{~Hz}, 2 \mathrm{H}), 2.43-2.26(\mathrm{~m}, 3 \mathrm{H})$, 2.10-1.98 (m, 3H), $1.26(\mathrm{t}, J=8.0 \mathrm{~Hz}, 3 \mathrm{H}), 0.97(\mathrm{~s}, 3 \mathrm{H}), 0.87(\mathrm{~s}, 3 \mathrm{H}) ;{ }^{13} \mathrm{C}$ NMR $(100 \mathrm{MHz}$, $\left.\mathrm{CDCl}_{3}\right) \delta 192.6,161.1,146.6,144.5,140.2,140.1,129.6,129.1,129.0,128.9,126.4,126.3,125.2$, 119.7, 116.7, 112.8, 76.4, 47.5, 41.8, 32.8, 28.4, 28.4, 27.5, 26.5, 25.3, 15.4. IR (KBr) v 3442, 2960, 1573, 1497, 1378, 1194, $754 \mathrm{~cm}^{-1}$. HRMS (ESI) calcd for $\mathrm{C}_{31} \mathrm{H}_{33} \mathrm{~N}_{2} \mathrm{O}[\mathrm{M}+\mathrm{H}]^{+} 449.2587$, found 449.2576 . 


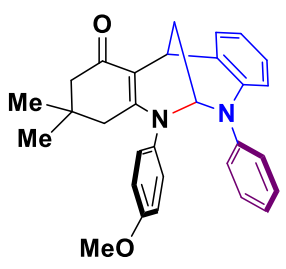

5-(4-methoxyphenyl)-3,3-dimethyl-7-phenyl-3,4,5,6,7,12-hexahydro-6,12-methanodibenzo[ $d, g][1$, 3]diazocin-1(2H)-one (7c)

Yellow oil obtained by column chromatography (petroleum ether/ethyl acetate $=5: 1$ ); $43.4 \mathrm{mg}, 96 \%$ yield; $\mathrm{dr}>20: 1$; reaction time $=5 \mathrm{~min} ;{ }^{1} \mathrm{H}$ NMR $\left(400 \mathrm{MHz}, \mathrm{CDCl}_{3}\right) \delta 7.42(\mathrm{~d}, J=8.0 \mathrm{~Hz}, 1 \mathrm{H})$, $7.15\left(\mathrm{dd}, J_{l}=J_{2}=8.0 \mathrm{~Hz}, 2 \mathrm{H}\right), 7.01(\mathrm{t}, J=8.0 \mathrm{~Hz}, 1 \mathrm{H}), 6.77\left(\mathrm{dd}, J_{l}=J_{2}=8.0 \mathrm{~Hz}, 6 \mathrm{H}\right), 6.64(\mathrm{t}, J=$ $8.0 \mathrm{~Hz}, 1 \mathrm{H}), 6.53(\mathrm{~d}, J=8.0 \mathrm{~Hz}, 2 \mathrm{H}), 5.19(\mathrm{~s}, 1 \mathrm{H}), 4.42(\mathrm{~s}, 1 \mathrm{H}), 3.73(\mathrm{~s}, 3 \mathrm{H}), 2.15\left(\mathrm{tt}, J_{l}=J_{2}=4.0\right.$ $\mathrm{Hz}, 1 \mathrm{H}), 2.12(\mathrm{~s}, 2 \mathrm{H}), 1.93\left(\mathrm{tt}, J_{I}=J_{2}=4.0 \mathrm{~Hz}, 1 \mathrm{H}\right), 1.86(\mathrm{~s}, 2 \mathrm{H}), 0.86(\mathrm{~s}, 3 \mathrm{H}), 0.75(\mathrm{~s}, 3 \mathrm{H}) ;{ }^{13} \mathrm{C}$ NMR (100 MHz, $\left.\mathrm{CDCl}_{3}\right) \delta 193.0,158.6,156.6,147.0,140.6,136.2,129.7,129.5,128.9,126.4$, $125.9,124.9,119.3,116.5,114.5,113.4,76.0,55.4,49.6,41.7,32.6,28.5,27.7,26.6,25.4$. IR $(\mathrm{KBr}) \vee 3442,2954,1619,1567,1499,755 \mathrm{~cm}^{-1}$. HRMS (ESI) calcd for $\mathrm{C}_{30} \mathrm{H}_{31} \mathrm{~N}_{2} \mathrm{O}_{2}[\mathrm{M}+\mathrm{H}]^{+}$ 451.2380, found 451.2382.

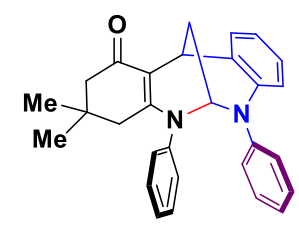

3,3-dimethyl-5,7-diphenyl-3,4,5,6,7,12-hexahydro-6,12-methanodibenzo[d,g][1,3]diazocin-1(2H)one $(\mathbf{7 d})$

Yellow oil obtained by column chromatography (petroleum ether/ethyl acetate $=5: 1$ ); $38.3 \mathrm{mg}, 91 \%$ yield; $\mathrm{dr}>20: 1$; reaction time $=5 \mathrm{~min} ;{ }^{1} \mathrm{H}$ NMR $\left(400 \mathrm{MHz}, \mathrm{CDCl}_{3}\right) \delta 7.51\left(\mathrm{dd}, J_{l}=J_{2}=4.0 \mathrm{~Hz}\right.$, 1H), 7.29-7.21 (m, 6H), $7.11(\mathrm{t}, J=8.0 \mathrm{~Hz}, 1 \mathrm{H}), 6.89$ (tt, $\left.J_{I}=J_{2}=8.0 \mathrm{~Hz}, 1 \mathrm{H}\right), 6.82(\mathrm{~d}, J=8.0 \mathrm{~Hz}$, 2H), $6.74\left(\mathrm{tt}, J_{I}=J_{2}=8.0 \mathrm{~Hz}, 2 \mathrm{H}\right), 6.58(\mathrm{~d}, J=8.0 \mathrm{~Hz}, 1 \mathrm{H}), 5.32(\mathrm{~s}, 1 \mathrm{H}), 4.50(\mathrm{~s}, 1 \mathrm{H}), 2.26\left(\mathrm{tt}, J_{I}\right.$ $\left.=J_{2}=4.0 \mathrm{~Hz}, 1 \mathrm{H}\right), 2.19(\mathrm{~s}, 2 \mathrm{H}), 2.04\left(\mathrm{tt}, J_{l}=J_{2}=4.0 \mathrm{~Hz}, 1 \mathrm{H}\right), 1.96(\mathrm{~d}, J=8.0 \mathrm{~Hz}, 2 \mathrm{H}), 0.95(\mathrm{~s}$, 3H), 0.84 (s, 3H); ${ }^{13} \mathrm{C}$ NMR (100 MHz, $\left.\mathrm{CDCl}_{3}\right) \delta$ 193.3, 155.2, 147.0, 143.7, 140.8, 129.6, 129.6, $129.4,129.0,127.3,126.9,126.0,125.1,119.2,116.2,114.2,75.9,50.2,41.9,32.9,28.6,27.8$, 26.8, 25.5. IR (KBr) v 3441, 2948, 1580, 1489, 1374, $758 \mathrm{~cm}^{-1}$. HRMS (ESI) calcd for $\mathrm{C}_{29} \mathrm{H}_{29} \mathrm{~N}_{2} \mathrm{O}$ $[\mathrm{M}+\mathrm{H}]^{+}$421.2274, found 421.2279 . 


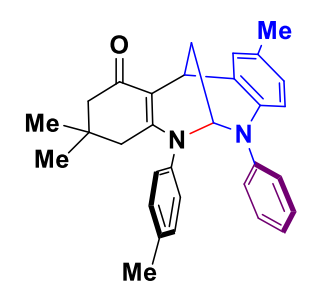

3,3,10-trimethyl-7-phenyl-5-(p-tolyl)-3,4,5,6,7,12-hexahydro-6,12-methanodibenzo[d,g][1,3]diazo cin-1(2H)-one (7e)

Yellow oil obtained by column chromatography (petroleum ether/ethyl acetate $=5: 1$ ); $43.5 \mathrm{mg}, 97 \%$ yield; $\mathrm{dr}>20: 1$; reaction time $=5 \mathrm{~min} ;{ }^{1} \mathrm{H}$ NMR $\left(400 \mathrm{MHz}, \mathrm{CDCl}_{3}\right) \delta 7.24(\mathrm{~d}, J=4.0 \mathrm{~Hz}, 1 \mathrm{H})$, $7.10(\mathrm{t}, J=8.0 \mathrm{~Hz}, 4 \mathrm{H}), 6.97(\mathrm{t}, J=8.0 \mathrm{~Hz}, 2 \mathrm{H}), 6.66(\mathrm{~d}, J=8.0 \mathrm{~Hz}, 3 \mathrm{H}), 6.48(\mathrm{~d}, J=8.0 \mathrm{~Hz}, 2 \mathrm{H})$, $5.22(\mathrm{~s}, 1 \mathrm{H}), 4.42(\mathrm{~s}, 1 \mathrm{H}), 2.31(\mathrm{~s}, 3 \mathrm{H}), 2.23(\mathrm{~d}, J=4.0 \mathrm{~Hz}, 2 \mathrm{H}), 2.21-2.17(\mathrm{~m}, 1 \mathrm{H}), 2.16(\mathrm{~s}, 3 \mathrm{H})$, 1.96-1.83 (m, 3H), 0.88 (s, 3H), $0.78(\mathrm{~s}, 3 \mathrm{H}) ;{ }^{13} \mathrm{C} \mathrm{NMR}\left(100 \mathrm{MHz}, \mathrm{CDCl}_{3}\right) \delta$ 192.7, 159.4, 147.3, $140.3,138.0,137.7,130.2,129.5,129.5,129.1,127.0,125.9,124.7,117.3,113.0,76.5,48.3,41.8$, 32.8, 28.7, 27.5, 26.4, 25.3, 21.1, 20.5. IR (KBr) v 3440, 2962, 1678, 1529, 1199, $811 \mathrm{~cm}^{-1}$. HRMS (ESI) calcd for $\mathrm{C}_{31} \mathrm{H}_{33} \mathrm{~N}_{2} \mathrm{O}[\mathrm{M}+\mathrm{H}]^{+} 449.2587$, found 449.2585 .

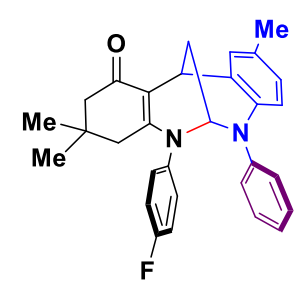

5-(4-fluorophenyl)-3,3,10-trimethyl-7-phenyl-3,4,5,6,7,12-hexahydro-6,12-methanodibenzo[ $d, g]$ $[1,3]$ diazocin-1(2H)-one (7f)

Yellow oil obtained by column chromatography (petroleum ether/ethyl acetate $=5: 1$ ); $43.9 \mathrm{mg}, 97 \%$ yield; $\mathrm{dr}>20: 1 ;$ reaction time $=5 \mathrm{~min} ;{ }^{1} \mathrm{H}$ NMR $\left(400 \mathrm{MHz}, \mathrm{CDCl}_{3}\right) \delta 7.24(\mathrm{~s}, 1 \mathrm{H}), 7.11(\mathrm{t}, J=8.0$ $\mathrm{Hz}, 2 \mathrm{H}), 6.98(\mathrm{t}, J=8.0 \mathrm{~Hz}, 4 \mathrm{H}), 6.70(\mathrm{~d}, J=8.0 \mathrm{~Hz}, 3 \mathrm{H}), 6.63(\mathrm{~d}, J=8.0 \mathrm{~Hz}, 1 \mathrm{H}), 6.46(\mathrm{~d}, J=8.0$ $\mathrm{Hz}, 1 \mathrm{H}), 5.18(\mathrm{~s}, 1 \mathrm{H}), 4.38(\mathrm{~s}, 1 \mathrm{H}), 2.17-2.11(\mathrm{~m}, 6 \mathrm{H}), 1.92-1.76(\mathrm{~m}, 3 \mathrm{H}), 0.87$ (s, 3H), $0.77(\mathrm{~s}$, 3H); ${ }^{13} \mathrm{C}$ NMR $\left(100 \mathrm{MHz}, \mathrm{CDCl}_{3}\right) \delta 193.1,161.4(\mathrm{~d}, J=246.0 \mathrm{~Hz}, 1 \mathrm{C}), 155.4,147.3,139.5$, 137.9, 129.8, 129.5, 129.4, 128.8, 126.8, 126.1, 124.7, 116.8, 116.2 (d, $J=22.0 \mathrm{~Hz}, 1 \mathrm{C}), 113.9$, 76.0, 49.8, 41.7, 32.7, 28.8, 27.5, 26.5, 25.4, 20.4. IR (KBr) v 3442, 2953, 1567, 1498, 1374, 1204 , $735 \mathrm{~cm}^{-1}$. HRMS (ESI) calcd for $\mathrm{C}_{30} \mathrm{H}_{30} \mathrm{FN}_{2} \mathrm{O}[\mathrm{M}+\mathrm{H}]^{+} 453.2337$, found 453.2334 . 


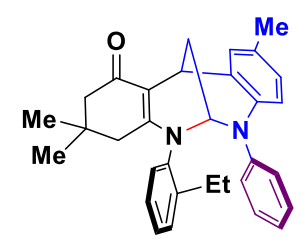

5-(2-ethylphenyl)-3,3,10-trimethyl-7-phenyl-3,4,5,6,7,12-hexahydro-6,12-methanodibenzo[d,g][1, 3]diazocin-1(2H)-one $(\mathbf{7 g})$

Yellow oil obtained by column chromatography (petroleum ether/ethyl acetate = 5:1); $41.7 \mathrm{mg}, 90 \%$ yield; $\mathrm{dr}>20: 1 ;$ reaction time $=5 \mathrm{~min} ;{ }^{1} \mathrm{H} \mathrm{NMR}\left(400 \mathrm{MHz}, \mathrm{CDCl}_{3}\right) \delta 7.42\left(\mathrm{td}, J_{l}=8.0 \mathrm{~Hz}, J_{2}=\right.$ $4.0 \mathrm{~Hz}, 2 \mathrm{H}), 7.36(\mathrm{~s}, 1 \mathrm{H}), 7.20(\mathrm{t}, J=8.0 \mathrm{~Hz}, 1 \mathrm{H}), 7.09(\mathrm{t}, J=8.0 \mathrm{~Hz}, 2 \mathrm{H}), 6.96(\mathrm{t}, J=8.0 \mathrm{~Hz}, 1 \mathrm{H})$, $6.87(\mathrm{~d}, J=8.0 \mathrm{~Hz}, 1 \mathrm{H}), 6.77(\mathrm{~d}, J=8.0 \mathrm{~Hz}, 1 \mathrm{H}), 6.69(\mathrm{~d}, J=8.0 \mathrm{~Hz}, 1 \mathrm{H}), 6.56(\mathrm{~d}, J=8.0 \mathrm{~Hz}$, 2H), $4.97(\mathrm{~s}, 1 \mathrm{H}), 4.58(\mathrm{~s}, 1 \mathrm{H}), 2.58(\mathrm{q}, J=8.0 \mathrm{~Hz}, 2 \mathrm{H}), 2.32-2.26(\mathrm{~m}, 6 \mathrm{H}), 2.03(\mathrm{~d}, J=16.0 \mathrm{~Hz}$, 2H), $1.72(\mathrm{~d}, J=16.0 \mathrm{~Hz}, 1 \mathrm{H}), 1.29(\mathrm{t}, J=8.0 \mathrm{~Hz}, 3 \mathrm{H}), 0.99(\mathrm{~s}, 3 \mathrm{H}), 0.86(\mathrm{~s}, 3 \mathrm{H}) ;{ }^{13} \mathrm{C} \mathrm{NMR}(100$ $\left.\mathrm{MHz}, \mathrm{CDCl}_{3}\right) \delta 192.3,158.1,148.0,141.0,140.3,137.2,130.9,130.4,129.6,129.3,128.8,126.9$ $126.9,124.7,124.0,118.6,112.5,75.7,48.9,41.3,32.8,29.4,27.1,26.1,24.6,23.2,21.5,14.2$, one carbon missing in the aromatic region. IR (KBr) $v 3440,2957,1562,1491,1377,771 \mathrm{~cm}^{-1}$. HRMS (ESI) calcd for $\mathrm{C}_{32} \mathrm{H}_{35} \mathrm{~N}_{2} \mathrm{O}[\mathrm{M}+\mathrm{H}]^{+} 463.2744$, found 463.2741.

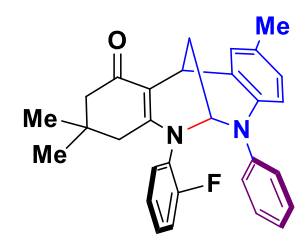

5-(2-fluorophenyl)-3,3,10-trimethyl-7-phenyl-3,4,5,6,7,12-hexahydro-6,12-methanodibenzo[d,g] $[1,3]$ diazocin-1(2H)-one $(\mathbf{7 h})$

Yellow oil obtained by column chromatography (petroleum ether/ethyl acetate $=5: 1$ ); $40.4 \mathrm{mg}, 89 \%$ yield; $\mathrm{dr}=3: 1 ;$ reaction time $=5 \mathrm{~min} ;{ }^{1} \mathrm{H} \mathrm{NMR}\left(400 \mathrm{MHz}, \mathrm{CDCl}_{3}\right) \delta 7.34-7.13(\mathrm{~m}, 5 \mathrm{H}), 7.10-6.93$ $(\mathrm{m}, 3 \mathrm{H}), 6.84(\mathrm{~d}, J=8.0 \mathrm{~Hz}, 1 \mathrm{H}), 6.70\left(\mathrm{dd}, J_{l}=J_{2}=8.0 \mathrm{~Hz}, 1 \mathrm{H}\right), 6.56(\mathrm{t}, J=8.0 \mathrm{~Hz}, 2 \mathrm{H}), 5.21(\mathrm{~s}$, 1H), $4.47(\mathrm{~s}, 1 \mathrm{H}), 2.29-2.18(\mathrm{~m}, 6 \mathrm{H}), 2.12-2.07(\mathrm{~m}, 1 \mathrm{H}), 1.99(\mathrm{~d}, J=16.0 \mathrm{~Hz}, 1 \mathrm{H}), 1.80(\mathrm{~d}, J=$ 16.0 Hz, 1H), $0.96(\mathrm{~s}, 3 \mathrm{H}), 0.87(\mathrm{~s}, 3 \mathrm{H}) ;{ }^{13} \mathrm{C} \mathrm{NMR}\left(100 \mathrm{MHz}, \mathrm{CDCl}_{3}\right) \delta 193.4,158.6(\mathrm{~d}, J=247.0$ $\mathrm{Hz}, 1 \mathrm{C}), 155.6,147.2,138.0,131.8,129.8,129.5,129.5,128.9$ (d, $J=38.0 \mathrm{~Hz}, 1 \mathrm{C}), 126.8,126.2$, $125.5,124.7,124.5(\mathrm{~d}, J=4.0 \mathrm{~Hz}, 1 \mathrm{C}), 116.7,116.4(\mathrm{~d}, J=20.0 \mathrm{~Hz}, 1 \mathrm{C}), 113.6,75.1,49.8,41.2$, 32.7, 29.1, 27.4, 26.5, 25.3, 20.4. IR (KBr) v 3443, 2951, 1578, 1497, 1378, $761 \mathrm{~cm}^{-1}$. HRMS (ESI) calcd for $\mathrm{C}_{30} \mathrm{H}_{30} \mathrm{FN}_{2} \mathrm{O}[\mathrm{M}+\mathrm{H}]^{+} 453.2337$, found 453.2333. 


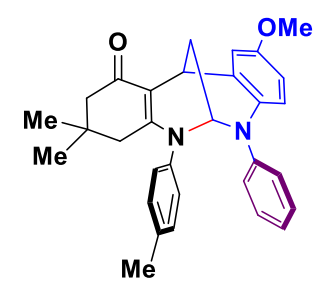

10-methoxy-3,3-dimethyl-7-phenyl-5-( $p$-tolyl)-3,4,5,6,7,12-hexahydro-6,12-methanodibenzo[ $d, g]$ $[1,3]$ diazocin-1 $(2 H)$-one $(7 \mathbf{i})$

Yellow oil obtained by column chromatography (petroleum ether/ethyl acetate $=5: 1$ ); $44.9 \mathrm{mg}, 97 \%$ yield; $\mathrm{dr}>20: 1$;reaction time $=5 \mathrm{~min} ;{ }^{1} \mathrm{H}$ NMR $\left(400 \mathrm{MHz}, \mathrm{CDCl}_{3}\right) \delta$ 7.18-7.09 (m, 5H), $7.01(\mathrm{t}, J$ $=8.0 \mathrm{~Hz}, 2 \mathrm{H}), 6.70(\mathrm{~d}, J=12.0 \mathrm{~Hz}, 3 \mathrm{H}), 6.61(\mathrm{~d}, J=12.0 \mathrm{~Hz}, 1 \mathrm{H}), 6.52\left(\mathrm{dd}, J_{l}=J_{2}=4.0 \mathrm{~Hz}, 1 \mathrm{H}\right)$, $5.23(\mathrm{~s}, 1 \mathrm{H}), 4.48(\mathrm{~s}, 1 \mathrm{H}), 3.76(\mathrm{~s}, 3 \mathrm{H}), 2.39(\mathrm{~s}, 3 \mathrm{H}), 2.26(\mathrm{~d}, J=16.0 \mathrm{~Hz}, 2 \mathrm{H}), 2.18(\mathrm{~s}, 1 \mathrm{H})$, 2.02-1.86 (m, 3H), 0.95 (s, 3H), 0.84 (s, 3H); ${ }^{13} \mathrm{C}$ NMR (100 MHz, $\left.\mathrm{CDCl}_{3}\right) \delta$ 192.7, 156.1, 153.3, 147.9, 140.7, 137.4, 133.8, 131.5, 130.1, 129.3, 125.4, 124.0, 119.1, 113.2, 112.8, 76.3, 55.6, 49.9, 41.6, 32.8, 28.7, 27.7, 26.8, 25.1, 21.1. IR (KBr) v3442, 2950, 1567, 1498, 1378, 1203, $764 \mathrm{~cm}^{-1}$. HRMS (ESI) calcd for $\mathrm{C}_{31} \mathrm{H}_{33} \mathrm{~N}_{2} \mathrm{O}_{2}[\mathrm{M}+\mathrm{H}]^{+} 465.2537$, found 465.2540 .

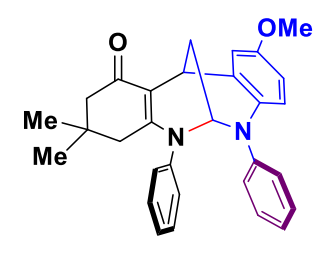

10-methoxy-3,3-dimethyl-5,7-diphenyl-3,4,5,6,7,12-hexahydro-6,12-methanodibenzo[ $d, g][1,3]$ dia zocin-1(2H)-one $(\mathbf{7 j})$

Yellow oil obtained by column chromatography (petroleum ether/ethyl acetate = 5:1); $40.9 \mathrm{mg}, 91 \%$ yield; $\mathrm{dr}>20: 1$; reaction time $=5 \mathrm{~min} ;{ }^{1} \mathrm{H} \mathrm{NMR}\left(400 \mathrm{MHz}, \mathrm{CDCl}_{3}\right) \delta 7.34(\mathrm{t}, J=8.0 \mathrm{~Hz}, 3 \mathrm{H})$, $7.15(\mathrm{t}, J=8.0 \mathrm{~Hz}, 2 \mathrm{H}), 7.10(\mathrm{~d}, J=4.0 \mathrm{~Hz}, 1 \mathrm{H}), 7.01(\mathrm{t}, J=8.0 \mathrm{~Hz}, 3 \mathrm{H}), 6.69(\mathrm{~d}, J=8.0 \mathrm{~Hz}, 2 \mathrm{H})$, $6.60(\mathrm{~d}, J=8.0 \mathrm{~Hz}, 1 \mathrm{H}), 6.52\left(\mathrm{dd}, J_{l}=J_{2}=4.0 \mathrm{~Hz}, 1 \mathrm{H}\right), 5.26(\mathrm{~s}, 1 \mathrm{H}), 4.49(\mathrm{~s}, 1 \mathrm{H}), 3.76(\mathrm{~s}, 3 \mathrm{H})$, $2.27\left(\mathrm{tt}, J_{l}=J_{2}=4.0 \mathrm{~Hz}, 1 \mathrm{H}\right), 2.19(\mathrm{~d}, J=4.0 \mathrm{~Hz}, 2 \mathrm{H}), 2.02-1.95(\mathrm{~m}, 2 \mathrm{H}), 1.89(\mathrm{~d}, J=16.0 \mathrm{~Hz}$, 1H), 0.95 (s, 3H), 0.84 (s, 3H); ${ }^{13} \mathrm{C}$ NMR (100 MHz, $\left.\mathrm{CDCl}_{3}\right) \delta$ 192.9, 155.7, 153.3, 147.8, 143.4, 133.8, 131.4, 129.5, 129.4, 127.5, 125.5, 124.1, 119.0, 113.5, 113.2, 112.9, 76.3, 55.6, 49.9, 41.7, 32.8, 28.8, 27.6, 26.9, 25.2. IR (KBr) v3441, 2953, 1561, 1491, 1203, 1048, $761 \mathrm{~cm}^{-1}$. HRMS (ESI) calcd for $\mathrm{C}_{30} \mathrm{H}_{31} \mathrm{~N}_{2} \mathrm{O}_{2}[\mathrm{M}+\mathrm{H}]^{+} 451.2380$, found 451.2378 . 


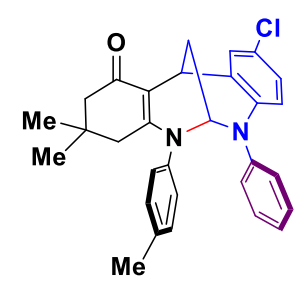

10-chloro-3,3-dimethyl-7-phenyl-5-(p-tolyl)-3,4,5,6,7,12-hexahydro-6,12-methanodibenzo[ $d, g][1$, 3]diazocin-1(2H)-one (7k)

Yellow oil obtained by column chromatography (petroleum ether/ethyl acetate $=5: 1$ ); $45.1 \mathrm{mg}, 96 \%$ yield; $\mathrm{dr}>20: 1$; reaction time $=5 \mathrm{~min} ;{ }^{1} \mathrm{H} \mathrm{NMR}\left(400 \mathrm{MHz}, \mathrm{CDCl}_{3}\right) \delta 7.41(\mathrm{~d}, J=4.0 \mathrm{~Hz}, 1 \mathrm{H})$, $7.16(\mathrm{t}, J=8.0 \mathrm{~Hz}, 3 \mathrm{H}), 7.04(\mathrm{t}, J=8.0 \mathrm{~Hz}, 3 \mathrm{H}), 6.75-6.72(\mathrm{~m}, 3 \mathrm{H}), 6.42(\mathrm{~d}, J=8.0 \mathrm{~Hz}, 2 \mathrm{H}), 5.21$ $(\mathrm{s}, 1 \mathrm{H}), 4.37(\mathrm{~s}, 1 \mathrm{H}), 2.28(\mathrm{~s}, 3 \mathrm{H}), 2.15(\mathrm{~s}, 2 \mathrm{H}), 2.12\left(\mathrm{tt}, J_{I}=J_{2}=4.0 \mathrm{~Hz}, 1 \mathrm{H}\right), 1.94\left(\mathrm{tt}, J_{I}=J_{2}=4.0\right.$ $\mathrm{Hz}, 1 \mathrm{H}), 1.88(\mathrm{~d}, J=4.0 \mathrm{~Hz}, 2 \mathrm{H}), 0.87$ (s, 3H), 0.78 (s, 3H); ${ }^{13} \mathrm{C} \mathrm{NMR}\left(100 \mathrm{MHz}, \mathrm{CDCl}_{3}\right) \delta 193.1$, 157.0, 146.5, 140.6, 139.5, 137.6, 131.1, 130.1, 129.7, 128.4, 126.6, 126.0, 125.4, 123.9, 117.4, 112.9, 75.7, 49.4, 41.8, 32.8, 28.5, 27.7, 26.7, 25.2, 21.0. IR (KBr) v 3440, 2953, 1575, 1481, 1417 , 1378, 1189, $763 \mathrm{~cm}^{-1}$. HRMS (ESI) calcd for $\mathrm{C}_{30} \mathrm{H}_{30} \mathrm{ClN}_{2} \mathrm{O}[\mathrm{M}+\mathrm{H}]^{+} 469.2041$, found 469.2048.

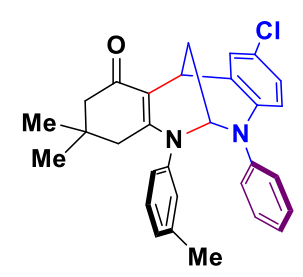

10-chloro-3,3-dimethyl-7-phenyl-5-( $m$-tolyl)-3,4,5,6,7,12-hexahydro-6,12-methanodibenzo[d, $g][1$, 3]diazocin-1(2H)-one (7l)

Yellow oil obtained by column chromatography (petroleum ether/ethyl acetate = 5:1); $42.1 \mathrm{mg}, 90 \%$ yield; $\mathrm{dr}>20: 1$; reaction time $=5 \mathrm{~min} ;{ }^{1} \mathrm{H} \mathrm{NMR}\left(400 \mathrm{MHz}, \mathrm{CDCl}_{3}\right) \delta 7.41(\mathrm{~d}, J=4.0 \mathrm{~Hz}, 1 \mathrm{H})$, $7.18(\mathrm{t}, J=8.0 \mathrm{~Hz}, 4 \mathrm{H}), 7.08(\mathrm{~d}, J=8.0 \mathrm{~Hz}, 1 \mathrm{H}), 7.04(\mathrm{t}, J=8.0 \mathrm{~Hz}, 1 \mathrm{H}), 6.76\left(\mathrm{dd}, J_{I}=J_{2}=4.0\right.$ $\mathrm{Hz}, 4 \mathrm{H}), 6.43(\mathrm{~d}, J=12.0 \mathrm{~Hz}, 1 \mathrm{H}), 5.26(\mathrm{~s}, 1 \mathrm{H}), 4.37(\mathrm{~s}, 1 \mathrm{H}), 2.20-2.11(\mathrm{~m}, 6 \mathrm{H}), 1.97\left(\mathrm{tt}, J_{l}=J_{2}=\right.$ $4.0 \mathrm{~Hz}, 1 \mathrm{H}), 1.91$ (s, 2H), 0.88 (s, 3H), $0.80(\mathrm{~s}, 3 \mathrm{H}) ;{ }^{13} \mathrm{C} \mathrm{NMR}\left(100 \mathrm{MHz}, \mathrm{CDCl}_{3}\right) \delta$ 193.3, 157.7, $146.3,143.0,139.7,139.4,130.8,129.8,129.3,128.5,128.4,126.8,126.2,125.6,123.8,117.1$, 116.9, 112.7, 75.6, 49.0, 41.9, 32.8, 28.5, 27.7, 26.7, 25.3, 21.2. IR (KBr) v 3442, 2955, 1567, 1486, 1378, $731 \mathrm{~cm}^{-1}$. HRMS (ESI) calcd for $\mathrm{C}_{30} \mathrm{H}_{30} \mathrm{ClN}_{2} \mathrm{O}[\mathrm{M}+\mathrm{H}]^{+} 469.2041$, found 469.2046 . 


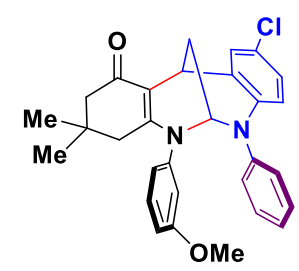

10-chloro-5-(3-methoxyphenyl)-3,3-dimethyl-7-phenyl-3,4,5,6,7,12-hexahydro-6,12-methanodibe $\mathrm{nzo}[d, g][1,3]$ diazocin-1(2H)-one $(\mathbf{7 m})$

Yellow oil obtained by column chromatography (petroleum ether/ethyl acetate $=5: 1$ ); $41.9 \mathrm{mg}, 86 \%$ yield; $\mathrm{dr}>20: 1$; reaction time $=5 \mathrm{~min} ;{ }^{1} \mathrm{H} \mathrm{NMR}\left(400 \mathrm{MHz}, \mathrm{CDCl}_{3}\right) \delta 7.42(\mathrm{~d}, J=4.0 \mathrm{~Hz}, 1 \mathrm{H})$, $7.21\left(\mathrm{dd}, J_{l}=8.0 \mathrm{~Hz}, J_{2}=4.0 \mathrm{~Hz}, 2 \mathrm{H}\right), 7.14-7.07(\mathrm{~m}, 2 \mathrm{H}), 6.83(\mathrm{~d}, J=8.0 \mathrm{~Hz}, 2 \mathrm{H}), 6.77-6.73(\mathrm{~m}$, 2H), $6.38(\mathrm{~d}, J=8.0 \mathrm{~Hz}, 3 \mathrm{H}), 5.24(\mathrm{~s}, 1 \mathrm{H}), 4.35(\mathrm{~s}, 1 \mathrm{H}), 3.60(\mathrm{~s}, 3 \mathrm{H}), 2.13-2.10(\mathrm{~m}, 3 \mathrm{H}), 1.97-1.92$ $(\mathrm{m}, 3 \mathrm{H}), 0.89$ (s, 3H), $0.80(\mathrm{~s}, 3 \mathrm{H}) ;{ }^{13} \mathrm{C} \mathrm{NMR}\left(100 \mathrm{MHz}, \mathrm{CDCl}_{3}\right) \delta 193.4,160.4,155.2,146.4$, $144.7,139.8,130.9,130.0,129.9,128.4,127.2,126.0,125.6,123.7,116.8,116.8,113.9,113.5$, 75.4, 55.4, 50.2, 41.9, 32.9, 28.5, 27.9, 26.9, 25.3. IR (KBr) v 3443, 2952, 1627, 1572, 1486, 1378 $775 \mathrm{~cm}^{-1}$. HRMS (ESI) calcd for $\mathrm{C}_{30} \mathrm{H}_{30} \mathrm{ClN}_{2} \mathrm{O}_{2}[\mathrm{M}+\mathrm{H}]^{+} 485.1990$, found 485.1997 .

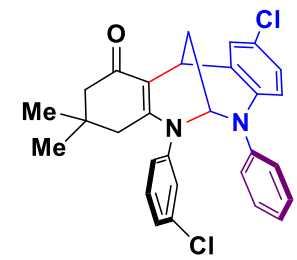

10-chloro-5-(3-chlorophenyl)-3,3-dimethyl-7-phenyl-3,4,5,6,7,12-hexahydro-6,12-methanodibenz $\mathrm{o}[d, g][1,3]$ diazocin-1(2H)-one $(\mathbf{7 n})$

Yellow oil obtained by column chromatography (petroleum ether/ethyl acetate $=5: 1$ ); $41.2 \mathrm{mg}, 84 \%$ yield; $\mathrm{dr}>20: 1$; reaction time $=5 \mathrm{~min} ;{ }^{1} \mathrm{H} \mathrm{NMR}\left(400 \mathrm{MHz}, \mathrm{CDCl}_{3}\right) \delta 7.41(\mathrm{~s}, 1 \mathrm{H}), 7.25-7.12$ $(\mathrm{m}, 6 \mathrm{H}), 6.84(\mathrm{~d}, J=8.0 \mathrm{~Hz}, 2 \mathrm{H}), 6.76(\mathrm{~d}, J=8.0 \mathrm{~Hz}, 2 \mathrm{H}), 6.40(\mathrm{~d}, J=8.0 \mathrm{~Hz}, 1 \mathrm{H}), 5.25(\mathrm{~s}, 1 \mathrm{H})$, $4.33(\mathrm{~s}, 1 \mathrm{H}), 2.12(\mathrm{~d}, J=12.0 \mathrm{~Hz}, 3 \mathrm{H}), 1.96(\mathrm{~d}, J=12.0 \mathrm{~Hz}, 1 \mathrm{H}), 1.89$ (s, 2H), 0.89 (s, 3H), 0.80 (s, $3 \mathrm{H}) ;{ }^{13} \mathrm{C} \mathrm{NMR}\left(100 \mathrm{MHz}, \mathrm{CDCl}_{3}\right) \delta 193.6,154.5,146.1,144.7,139.7,134.9,130.6,130.3,129.9$, $128.4,128.3,127.5,127.2,126.1,126.0,123.7,116.6,114.3,75.4,50.0,42.0,32.9,28.5,27.8$, 26.8, 25.4. IR (KBr) v 3441, 2955, 1629, 1571, 1483, 1377, $807 \mathrm{~cm}^{-1}$. HRMS (ESI) calcd for $\mathrm{C}_{29} \mathrm{H}_{27} \mathrm{Cl}_{2} \mathrm{~N}_{2} \mathrm{O}[\mathrm{M}+\mathrm{H}]^{+} 489.1495$, found 489.1476 . 


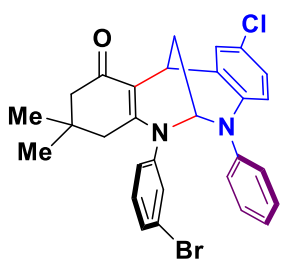

5-(3-bromophenyl)-10-chloro-3,3-dimethyl-7-phenyl-3,4,5,6,7,12-hexahydro-6,12-methanodibenz $\mathrm{o}[d, g][1,3]$ diazocin-1(2H)-one $(7 \mathbf{o})$

Yellow oil obtained by column chromatography (petroleum ether/ethyl acetate $=5: 1$ ); $46.0 \mathrm{mg}, 86 \%$ yield; $\mathrm{dr}>20: 1$; reaction time $=5 \mathrm{~min} ;{ }^{1} \mathrm{H} \mathrm{NMR}\left(400 \mathrm{MHz}, \mathrm{CDCl}_{3}\right) \delta 7.40(\mathrm{~d}, J=4.0 \mathrm{~Hz}, 2 \mathrm{H})$, $7.33(\mathrm{~d}, J=8.0 \mathrm{~Hz}, 1 \mathrm{H}), 7.24(\mathrm{t}, J=8.0 \mathrm{~Hz}, 2 \mathrm{H}), 7.11(\mathrm{q}, J=8.0 \mathrm{~Hz}, 2 \mathrm{H}), 6.84(\mathrm{~d}, J=8.0 \mathrm{~Hz}, 2 \mathrm{H})$, $6.77\left(\mathrm{dd}, J_{1}=J_{2}=4.0 \mathrm{~Hz}, 2 \mathrm{H}\right), 6.42(\mathrm{~d}, J=8.0 \mathrm{~Hz}, 1 \mathrm{H}), 5.27(\mathrm{~s}, 1 \mathrm{H}), 4.34(\mathrm{~s}, 1 \mathrm{H}), 2.20(\mathrm{~s}, 2 \mathrm{H})$, $2.14\left(\mathrm{tt}, J_{l}=J_{2}=4.0 \mathrm{~Hz}, 1 \mathrm{H}\right), 1.98\left(\mathrm{tt}, J_{l}=J_{2}=4.0 \mathrm{~Hz}, 1 \mathrm{H}\right), 1.90(\mathrm{~s}, 2 \mathrm{H}), 0.89(\mathrm{~s}, 3 \mathrm{H}), 0.81(\mathrm{~s}, 3 \mathrm{H})$; ${ }^{13} \mathrm{C}$ NMR $\left(100 \mathrm{MHz}, \mathrm{CDCl}_{3}\right) \delta 194.1,156.5,146.0,144.5,139.5,130.8,130.6,130.4,130.0$, $128.3,127.1,126.3,126.1,123.9,122.9,116.8,113.8,75.6,49.1,42.0,33.0,28.4,27.8,26.8,25.5$ one carbon missing in the aromatic region. IR $(\mathrm{KBr}) v 3443,2954,1628,1568,1483,1378,733$ $\mathrm{cm}^{-1}$. HRMS (ESI) calcd for $\mathrm{C}_{29} \mathrm{H}_{27} \mathrm{BrClN}_{2} \mathrm{O}[\mathrm{M}+\mathrm{H}]^{+}$533.0990, found 533.0994.

\section{Experimental data for the formation of 9}
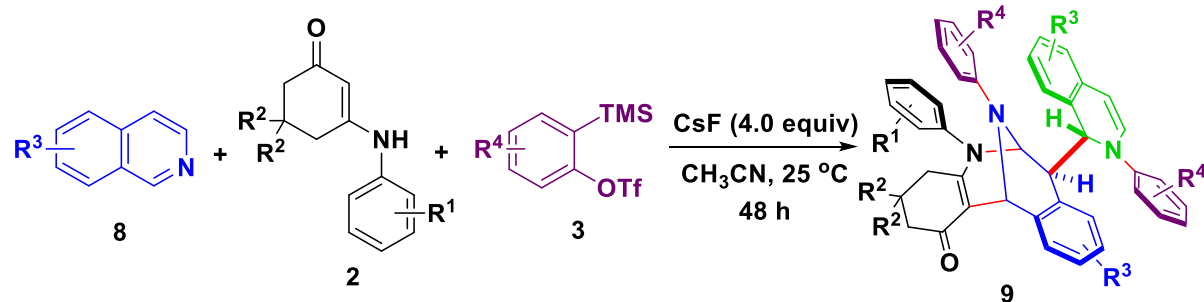

General procedure: To a $5.0 \mathrm{~mL}$ vial were successively added isoquinolines $8(0.30 \mathrm{mmol})$, enaminones $2(0.15 \mathrm{mmol})$, aryne precursors $3(0.60 \mathrm{mmol}), \mathrm{CsF}(0.60 \mathrm{mmol})$ and $1.0 \mathrm{~mL}$ of $\mathrm{CH}_{3} \mathrm{CN}$. The resulting mixture was stirred at $60{ }^{\circ} \mathrm{C}$ in oil bath. Upon completion of the reaction (monitoring by TLC), the products 9 were precipitated from the reaction mixtures and only a filtration was needed to purify them.

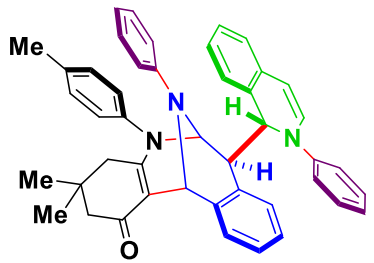

3,3-dimethyl-13-phenyl-7-(2-phenyl-1,2-dihydroisoquinolin-1-yl)-5-( $p$-tolyl)-3,4,5,6,7,12-hexahy dro-6,12-epiminodibenzo[b,e $]$ azocin-1(2H)-one (9a) 
White solid obtained by filtration of the precipitate; $82.2 \mathrm{mg}, 86 \%$ yield; $\mathrm{dr}>20: 1$; reaction time $=$ $6 \mathrm{~h} ; \mathrm{mp} 243.2-243.8{ }^{\circ} \mathrm{C} ;{ }^{1} \mathrm{H}$ NMR $\left(400 \mathrm{MHz}, \mathrm{CDCl}_{3}\right) \delta 7.60(\mathrm{~d}, J=8.0 \mathrm{~Hz}, 1 \mathrm{H}), 7.33(\mathrm{t}, J=8.0$ $\mathrm{Hz}, 2 \mathrm{H}), 7.21(\mathrm{t}, J=8.0 \mathrm{~Hz}, 2 \mathrm{H}), 7.14(\mathrm{q}, J=8.0 \mathrm{~Hz}, 2 \mathrm{H}), 7.07-6.88(\mathrm{~m}, 9 \mathrm{H}), 6.75(\mathrm{q}, J=8.0 \mathrm{~Hz}$, 2H), $6.59(\mathrm{~d}, J=8.0 \mathrm{~Hz}, 1 \mathrm{H}), 6.52(\mathrm{br}, 2 \mathrm{H}), 6.34(\mathrm{~d}, J=8.0 \mathrm{~Hz}, 2 \mathrm{H}), 6.28(\mathrm{~s}, 1 \mathrm{H}), 5.85(\mathrm{~d}, J=8.0$ Hz, 1H), 5.64 (s, 1H), 5.23 (d, $J=12.0 \mathrm{~Hz}, 1 \mathrm{H}), 3.26(\mathrm{~d}, J=12.0 \mathrm{~Hz}, 1 \mathrm{H}), 2.32$ (s, 3H), 2.07-1.93 $(\mathrm{m}, 3 \mathrm{H}), 1.51(\mathrm{~d}, J=16.0 \mathrm{~Hz}, 1 \mathrm{H}), 0.70(\mathrm{~s}, 3 \mathrm{H}), 0.45(\mathrm{~s}, 3 \mathrm{H}) ;{ }^{13} \mathrm{C} \mathrm{NMR}\left(100 \mathrm{MHz}, \mathrm{CDCl}_{3}\right) \delta 191.7$, $155.3,148.3,145.3,138.8,137.8,136.7,131.3,130.5,130.1,130.0,129.4,129.2,128.9,128.5$, 127.7, 127.3, 127.2, 126.9, 126.0, 125.0, 123.6, 121.3, 121.0, 117.9, 117.0, 109.7, 105.9, 71.9, 63.2, 52.4, 49.5, 43.3, 41.1, 33.4, 29.8, 26.2, 21.0. IR (KBr) v 3447, 2953, 1610, 1564, 1501, 1259, $761 \mathrm{~cm}^{-1}$. HRMS (ESI) calcd for $\mathrm{C}_{45} \mathrm{H}_{42} \mathrm{~N}_{3} \mathrm{O}[\mathrm{M}+\mathrm{H}]^{+} 640.3322$, found 640.3323 .

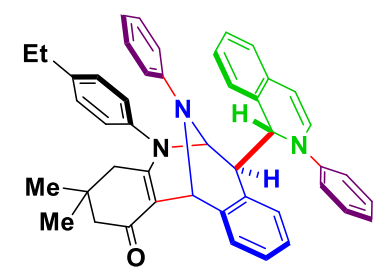

5-(4-ethylphenyl)-3,3-dimethyl-13-phenyl-7-(2-phenyl-1,2-dihydroisoquinolin-1-yl)-3,4,5,6,7,12hexahydro-6,12-epiminodibenzo[b,e]azocin-1(2H)-one (9b)

White solid obtained by filtration of the precipitate; $85.6 \mathrm{mg}, 87 \%$ yield; $\mathrm{dr}>20: 1$; reaction time $=$ $2 \mathrm{~h} ; \mathrm{mp} 255.9-256.6{ }^{\circ} \mathrm{C} ;{ }^{1} \mathrm{H}$ NMR $\left(400 \mathrm{MHz}, \mathrm{CDCl}_{3}\right) \delta 7.52(\mathrm{~d}, J=8.0 \mathrm{~Hz}, 1 \mathrm{H}), 7.25(\mathrm{t}, J=8.0$ $\mathrm{Hz}, 2 \mathrm{H}), 7.15(\mathrm{t}, J=8.0 \mathrm{~Hz}, 2 \mathrm{H}), 7.06(\mathrm{q}, J=8.0 \mathrm{~Hz}, 2 \mathrm{H}), 6.98(\mathrm{t}, J=8.0 \mathrm{~Hz}, 2 \mathrm{H}), 6.95-6.88(\mathrm{~m}$, $6 \mathrm{H}), 6.84(\mathrm{~d}, J=8.0 \mathrm{~Hz}, 2 \mathrm{H}), 6.68(\mathrm{t}, J=8.0 \mathrm{~Hz}, 2 \mathrm{H}), 6.51\left(\mathrm{dd}, J_{I}=J_{2}=4.0 \mathrm{~Hz}, 3 \mathrm{H}\right), 6.27(\mathrm{~d}, J=$ $8.0 \mathrm{~Hz}, 2 \mathrm{H}), 6.20(\mathrm{~s}, 1 \mathrm{H}), 5.75(\mathrm{~d}, J=4.0 \mathrm{~Hz}, 1 \mathrm{H}), 5.57$ (s, 1H), 5.14 (d, J = 8.0 Hz, 1H), 3.17 (d, $J=8.0 \mathrm{~Hz}, 1 \mathrm{H}), 2.54(\mathrm{q}, J=8.0 \mathrm{~Hz}, 2 \mathrm{H}), 1.92(\mathrm{q}, J=16.0 \mathrm{~Hz}, 2 \mathrm{H}), 1.46(\mathrm{~d}, J=16.0 \mathrm{~Hz}, 1 \mathrm{H})$, $1.17(\mathrm{t}, J=8.0 \mathrm{~Hz}, 3 \mathrm{H}), 0.63(\mathrm{~s}, 3 \mathrm{H}), 0.40(\mathrm{~s}, 3 \mathrm{H}) ;{ }^{13} \mathrm{C} \mathrm{NMR}\left(100 \mathrm{MHz}, \mathrm{CDCl}_{3}\right) \delta$ 191.7, 155.3, 148.4, 145.3, 143.1, 138.9, 138.0, 131.3, 130.6, 129.4, 129.3, 128.9, 128.9, 128.5, 127.7, 127.4, 127.2, 126.9, 126.1, 126.0, 125.0, 123.6, 121.4, 121.1, 117.9, 117.1, 109.6, 105.9, 72.1, 63.2, 52.4, 49.5, 42.3, 41.2, 33.5, 29.8, 28.4, 26.3, 15.6. IR (KBr) v 3442, 2956, 1607, 1559, 1498, 1390, 1257, $761 \mathrm{~cm}^{-1}$. HRMS (ESI) calcd for $\mathrm{C}_{46} \mathrm{H}_{43} \mathrm{~N}_{3} \mathrm{NaO}[\mathrm{M}+\mathrm{Na}]^{+} 676.3298$, found 676.3305 .

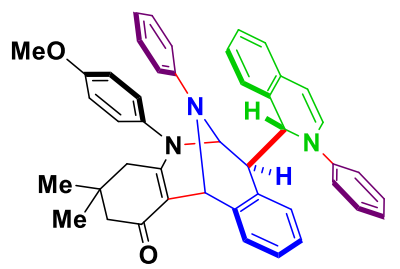


5-(4-methoxyphenyl)-3,3-dimethyl-13-phenyl-7-(2-phenyl-1,2-dihydroisoquinolin-1-yl)-3,4,5,6,7, 12-hexahydro-6,12-epiminodibenzo[b,e]azocin-1(2H)-one (9c)

White solid obtained by filtration of the precipitate; $88.7 \mathrm{mg}, 90 \%$ yield; $\mathrm{dr}>20: 1$; reaction time $=$ $2 \mathrm{~h} ; \mathrm{mp} 238.1-238.7{ }^{\circ} \mathrm{C} ;{ }^{1} \mathrm{H}$ NMR $\left(400 \mathrm{MHz}, \mathrm{CDCl}_{3}\right) \delta 7.52(\mathrm{~d}, J=4.0 \mathrm{~Hz}, 1 \mathrm{H}), 7.26(\mathrm{t}, J=8.0$ $\mathrm{Hz}, 2 \mathrm{H}), 7.13(\mathrm{t}, J=8.0 \mathrm{~Hz}, 2 \mathrm{H}), 7.06(\mathrm{t}, J=8.0 \mathrm{~Hz}, 2 \mathrm{H}), 7.00(\mathrm{~d}, J=8.0 \mathrm{~Hz}, 1 \mathrm{H}), 6.95-6.82(\mathrm{~m}$, 6H), 6.70-6.61 (m, 5H), $6.53(\mathrm{~d}, J=8.0 \mathrm{~Hz}, 1 \mathrm{H}), 6.45(\mathrm{br}, 1 \mathrm{H}), 6.27(\mathrm{~d}, J=8.0 \mathrm{~Hz}, 2 \mathrm{H}), 6.20(\mathrm{~s}$, $1 \mathrm{H}), 5.79(\mathrm{~d}, J=8.0 \mathrm{~Hz}, 1 \mathrm{H}), 5.50(\mathrm{~s}, 1 \mathrm{H}), 5.17(\mathrm{~d}, J=12.0 \mathrm{~Hz}, 1 \mathrm{H}), 3.72(\mathrm{~s}, 3 \mathrm{H}), 3.22(\mathrm{~d}, J=8.0$ $\mathrm{Hz}, 1 \mathrm{H}), 1.98-1.86(\mathrm{~m}, 3 \mathrm{H}), 1.43(\mathrm{~d}, J=16.0 \mathrm{~Hz}, 1 \mathrm{H}), 0.62(\mathrm{~s}, 3 \mathrm{H}), 0.40(\mathrm{~s}, 3 \mathrm{H}) ;{ }^{13} \mathrm{C}$ NMR $(100$ $\left.\mathrm{MHz}, \mathrm{CDCl}_{3}\right) \delta 191.6,158.3,155.6,148.4,145.3,138.8,133.2,131.3,130.5,129.5,129.3,129.2$, $128.9,128.5,127.7,127.4,127.3,126.9,126.1,126.0,125.0,123.6,121.3,121.1,117.8,117.1$, 109.4, 105.9, 72.2, 63.3, 55.5, 52.3, 49.4, 42.4, 41.0, 33.3, 29.7, 26.4. IR (KBr) v 3443, 2953, 1560, 1501, 1251, $760 \mathrm{~cm}^{-1}$. HRMS (ESI) calcd for $\mathrm{C}_{45} \mathrm{H}_{41} \mathrm{~N}_{3} \mathrm{NaO}_{2}[\mathrm{M}+\mathrm{Na}]^{+}$678.3091, found 678.3094

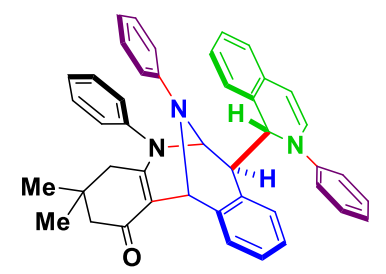

3,3-dimethyl-5,13-diphenyl-7-(2-phenyl-1,2-dihydroisoquinolin-1-yl)-3,4,5,6,7,12-hexahydro-6,1 2-epiminodibenzo[b,e]azocin-1(2H)-one (9d)

White solid obtained by filtration of the precipitate; $71.9 \mathrm{mg}, 77 \%$ yield; $\mathrm{dr}>20: 1$; reaction time $=$ $2 \mathrm{~h} ; \mathrm{mp} 183.7-184.6{ }^{\circ} \mathrm{C} ;{ }^{1} \mathrm{H} \mathrm{NMR}\left(400 \mathrm{MHz}, \mathrm{CDCl}_{3}\right) \delta 7.59(\mathrm{~d}, J=8.0 \mathrm{~Hz}, 1 \mathrm{H}), 7.35(\mathrm{t}, J=8.0$ $\mathrm{Hz}, 2 \mathrm{H}), 7.25-7.12(\mathrm{~m}, 7 \mathrm{H}), 7.06-6.88(\mathrm{~m}, 7 \mathrm{H}), 6.77(\mathrm{q}, J=8.0 \mathrm{~Hz}, 2 \mathrm{H}), 6.64(\mathrm{br}, 2 \mathrm{H}), 6.59(\mathrm{~d}, J$ $=4.0 \mathrm{~Hz}, 1 \mathrm{H}), 6.33(\mathrm{~s}, 1 \mathrm{H}), 6.30(\mathrm{~d}, J=4.0 \mathrm{~Hz}, 2 \mathrm{H}), 5.85(\mathrm{~d}, J=8.0 \mathrm{~Hz}, 1 \mathrm{H}), 5.67(\mathrm{~s}, 1 \mathrm{H}), 5.22(\mathrm{~d}$, $J=8.0 \mathrm{~Hz}, 1 \mathrm{H}), 3.23(\mathrm{~d}, J=12.0 \mathrm{~Hz}, 1 \mathrm{H}), 2.06\left(\mathrm{dd}, J_{l}=J_{2}=4.0 \mathrm{~Hz}, 2 \mathrm{H}\right), 1.96(\mathrm{~d}, J=16.0 \mathrm{~Hz}$, $1 \mathrm{H}), 1.51(\mathrm{~d}, J=16.0 \mathrm{~Hz}, 1 \mathrm{H}), 0.71(\mathrm{~s}, 3 \mathrm{H}), 0.46(\mathrm{~s}, 3 \mathrm{H}) ;{ }^{13} \mathrm{C} \mathrm{NMR}\left(100 \mathrm{MHz}, \mathrm{CDCl}_{3}\right) \delta 191.9$, 155.1, 148.4, 145.3, 140.6, 138.7, 131.3, 130.7, 129.6, 129.4, 129.3, 128.9, 128.6, 127.7, 127.4, $127.3,127.0,126.8,126.1,126.1,125.1,123.7,121.5,121.1,117.9,117.1,110.1,106.0,72.0$, 63.3, 52.5, 49.5, 42.2, 41.3, 33.6, 29.8, 26.2. IR (KBr) v 3451, 2954, 1603, 1558, 1493, 1390, 735 $\mathrm{cm}^{-1}$. HRMS (ESI) calcd for $\mathrm{C}_{44} \mathrm{H}_{40} \mathrm{~N}_{3} \mathrm{O}[\mathrm{M}+\mathrm{H}]^{+} 626.3166$, found 626.3189 . 


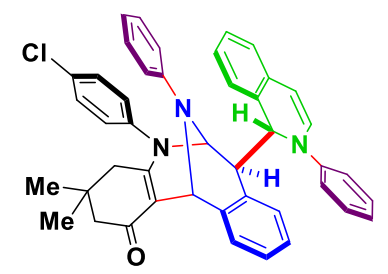

5-(4-chlorophenyl)-3,3-dimethyl-13-phenyl-7-(2-phenyl-1,2-dihydroisoquinolin-1-yl)-3,4,5,6,7,12 -hexahydro-6,12-epiminodibenzo[b,e]azocin-1(2H)-one $(\mathbf{9 e})$

White solid obtained by filtration of the precipitate; $91.1 \mathrm{mg}, 92 \%$ yield; $\mathrm{dr}>20: 1$; reaction time $=$ $2 \mathrm{~h} ; \mathrm{mp} 236.2-236.9{ }^{\circ} \mathrm{C} ;{ }^{1} \mathrm{H}$ NMR $\left(400 \mathrm{MHz}, \mathrm{CDCl}_{3}\right) \delta 7.58(\mathrm{~d}, J=8.0 \mathrm{~Hz}, 1 \mathrm{H}), 7.32(\mathrm{~d}, J=8.0$ $\mathrm{Hz}, 2 \mathrm{H}), 7.19-7.11(\mathrm{~m}, 6 \mathrm{H}), 7.05-6.96(\mathrm{~m}, 6 \mathrm{H}), 6.91(\mathrm{t}, J=8.0 \mathrm{~Hz}, 1 \mathrm{H}), 6.76(\mathrm{q}, J=8.0 \mathrm{~Hz}, 2 \mathrm{H})$, $6.61(\mathrm{~d}, J=8.0 \mathrm{~Hz}, 1 \mathrm{H}), 6.55(\mathrm{~d}, J=8.0 \mathrm{~Hz}, 2 \mathrm{H}), 6.34(\mathrm{~d}, J=8.0 \mathrm{~Hz}, 2 \mathrm{H}), 6.27(\mathrm{~s}, 1 \mathrm{H}), 5.89(\mathrm{~d}, J$ $=8.0 \mathrm{~Hz}, 1 \mathrm{H}), 5.65(\mathrm{~s}, 1 \mathrm{H}), 5.23(\mathrm{~d}, J=8.0 \mathrm{~Hz}, 1 \mathrm{H}), 3.21(\mathrm{~d}, J=8.0 \mathrm{~Hz}, 1 \mathrm{H}), 2.07-1.94(\mathrm{~m}, 3 \mathrm{H})$, $1.48(\mathrm{~d}, J=16.0 \mathrm{~Hz}, 1 \mathrm{H}), 0.72(\mathrm{~s}, 3 \mathrm{H}), 0.45(\mathrm{~s}, 3 \mathrm{H}) ;{ }^{13} \mathrm{C} \mathrm{NMR}\left(100 \mathrm{MHz}, \mathrm{CDCl}_{3}\right) \delta 192.1,154.4$, $148.2,145.2,139.1,138.5,132.3,131.4,130.6,129.6,129.3,129.2,128.9,128.6,127.6,127.5$, $127.4,127.0,126.2,126.0,125.1,123.7,121.6,121.2,117.9,117.0,110.7,105.9,72.0,63.2,52.4$, 49.5, 42.3, 41.3, 33.6, 29.9, 26.1. IR (KBr) v 3444, 2954, 1597, 1561, 1492, $759 \mathrm{~cm}^{-1}$. HRMS (ESI) calcd for $\mathrm{C}_{44} \mathrm{H}_{38} \mathrm{ClN}_{3} \mathrm{NaO}[\mathrm{M}+\mathrm{Na}]^{+}$682.2596, found 682.2603.

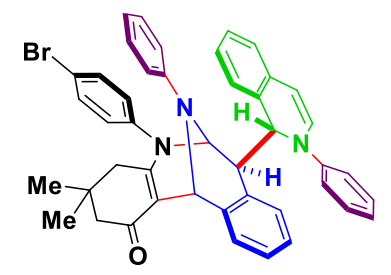

5-(4-bromophenyl)-3,3-dimethyl-13-phenyl-7-(2-phenyl-1,2-dihydroisoquinolin-1-yl)-3,4,5,6,7,12 -hexahydro-6,12-epiminodibenzo[b,e]azocin-1(2H)-one (9f)

White solid obtained by filtration of the precipitate; $99.8 \mathrm{mg}, 94 \%$ yield; $\mathrm{dr}>20: 1$; reaction time $=$ $2 \mathrm{~h} ; \mathrm{mp} 244.0-244.9{ }^{\circ} \mathrm{C} ;{ }^{1} \mathrm{H}$ NMR $\left(400 \mathrm{MHz}, \mathrm{CDCl}_{3}\right) \delta 7.50(\mathrm{~d}, J=8.0 \mathrm{~Hz}, 1 \mathrm{H}), 7.28-7.20(\mathrm{~m}$, 4H), 7.13-7.04 (m, 4H), 6.98-6.88 (m, 6H), $6.84(\mathrm{t}, J=8.0 \mathrm{~Hz}, 1 \mathrm{H}), 6.72-6.65(\mathrm{~m}, 2 \mathrm{H}), 6.54(\mathrm{dd}$, $\left.J_{l}=J_{2}=4.0 \mathrm{~Hz}, 1 \mathrm{H}\right), 6.41(\mathrm{~d}, J=8.0 \mathrm{~Hz}, 2 \mathrm{H}), 6.26(\mathrm{~d}, J=8.0 \mathrm{~Hz}, 2 \mathrm{H}), 6.19(\mathrm{~s}, 1 \mathrm{H}), 5.81(\mathrm{~d}, J=$ $8.0 \mathrm{~Hz}, 1 \mathrm{H}), 5.59(\mathrm{~s}, 1 \mathrm{H}), 5.16(\mathrm{~d}, J=8.0 \mathrm{~Hz}, 1 \mathrm{H}), 3.13(\mathrm{~d}, J=12.0 \mathrm{~Hz}, 1 \mathrm{H}), 2.00-1.87(\mathrm{~m}, 3 \mathrm{H})$, $1.41(\mathrm{~d}, J=16.0 \mathrm{~Hz}, 1 \mathrm{H}), 0.65(\mathrm{~s}, 3 \mathrm{H}), 0.37(\mathrm{~s}, 3 \mathrm{H}) ;{ }^{13} \mathrm{C} \mathrm{NMR}\left(100 \mathrm{MHz}, \mathrm{CDCl}_{3}\right) \delta 192.1,154.3$, $148.2,145.2,139.6,138.5,132.6,131.4,130.6,129.3,129.2,128.9,128.6,127.5,127.5,127.4$, 127.0, 126.2, 126.0, 125.1, 123.7, 121.6, 121.2, 120.2, 117.9, 117.1, 110.8, 105.9, 72.0, 63.2, 52.4, 
49.5, 42.3, 41.3, 33.6, 29.9, 26.1. IR (KBr) v 3448, 2954, 1593, 1561, 1491, 1387, $759 \mathrm{~cm}^{-1}$. HRMS (ESI) calcd for $\mathrm{C}_{44} \mathrm{H}_{38} \mathrm{BrN}_{3} \mathrm{NaO}[\mathrm{M}+\mathrm{Na}]^{+} 726.2090$, found 726.2096.

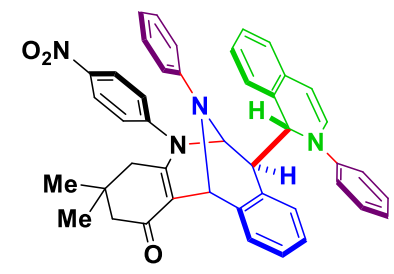

3,3-dimethyl-5-(4-nitrophenyl)-13-phenyl-7-(2-phenyl-1,2-dihydroisoquinolin-1-yl)-3,4,5,6,7,12hexahydro-6,12-epiminodibenzo[b,e]azocin-1(2H)-one (9g)

White solid obtained by filtration of the precipitate; $93.6 \mathrm{mg}, 93 \%$ yield; $\mathrm{dr}>20: 1$; reaction time $=$ $2 \mathrm{~h} ; \mathrm{mp} 226.7-227.5^{\circ} \mathrm{C} ;{ }^{1} \mathrm{H}$ NMR $\left(400 \mathrm{MHz}, \mathrm{CDCl}_{3}\right) \delta 7.91(\mathrm{~d}, J=8.0 \mathrm{~Hz}, 2 \mathrm{H}), 7.47(\mathrm{~d}, J=8.0$ $\mathrm{Hz}, 1 \mathrm{H}), 7.28(\mathrm{t}, J=8.0 \mathrm{~Hz}, 2 \mathrm{H}), 7.22(\mathrm{t}, J=8.0 \mathrm{~Hz}, 1 \mathrm{H}), 7.10-7.07(\mathrm{~m}, 3 \mathrm{H}), 7.05-6.99(\mathrm{~m}, 1 \mathrm{H})$, $6.92\left(\mathrm{dd}, J_{1}=4.0 \mathrm{~Hz}, J_{2}=8.0 \mathrm{~Hz}, 4 \mathrm{H}\right), 6.82(\mathrm{t}, J=8.0 \mathrm{~Hz}, 1 \mathrm{H}), 6.70\left(\mathrm{dd}, J_{l}=J_{2}=4.0 \mathrm{~Hz}, 2 \mathrm{H}\right)$, $6.55(\mathrm{t}, J=8.0 \mathrm{~Hz}, 3 \mathrm{H}), 6.24(\mathrm{~s}, 2 \mathrm{H}), 5.81(\mathrm{~d}, J=4.0 \mathrm{~Hz}, 2 \mathrm{H}), 5.15(\mathrm{~d}, J=8.0 \mathrm{~Hz}, 1 \mathrm{H}), 3.02(\mathrm{~d}, J$ $=8.0 \mathrm{~Hz}, 1 \mathrm{H}), 2.35-1.89(\mathrm{~m}, 5 \mathrm{H}), 1.44(\mathrm{~d}, J=16.0 \mathrm{~Hz}, 1 \mathrm{H}), 0.72(\mathrm{~s}, 3 \mathrm{H}), 0.32(\mathrm{~s}, 3 \mathrm{H}) ;{ }^{13} \mathrm{C} \mathrm{NMR}$ $\left(100 \mathrm{MHz}, \mathrm{CDCl}_{3}\right) \delta 192.9,152.7,147.9,146.5,145.1,144.7,137.7,131.5,130.8,129.5,128.9$, $128.8,128.6,127.7,127.5,127.5,127.1,126.5,126.0,125.3,124.8,123.9,122.2,121.3,120.8$, 118.0, 116.9, 113.9, 71.5, 63.1, 52.9, 49.6, 42.3, 42.0, 34.2, 30.2, 25.6. IR (KBr) v 3448, 2948, 1569, 1496, 1268, $759 \mathrm{~cm}^{-1}$. HRMS (ESI) calcd for $\mathrm{C}_{44} \mathrm{H}_{38} \mathrm{~N}_{4} \mathrm{NaO}_{3}[\mathrm{M}+\mathrm{Na}]^{+}$693.2836, found 693.2841

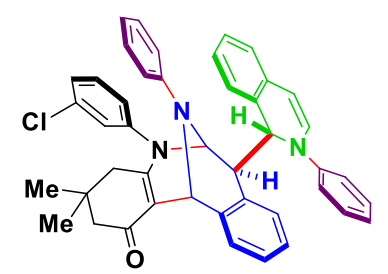

5-(3-chlorophenyl)-3,3-dimethyl-13-phenyl-7-(2-phenyl-1,2-dihydroisoquinolin-1-yl)-3,4,5,6,7,12 -hexahydro-6,12-epiminodibenzo[b,e]azocin-1(2H)-one (9h)

White solid obtained by filtration of the precipitate; $82.5 \mathrm{mg}, 83 \%$ yield; $\mathrm{dr}>20: 1$; reaction time $=$ $6 \mathrm{~h} ; \mathrm{mp} 191.5-192.3^{\circ} \mathrm{C} ;{ }^{1} \mathrm{H}$ NMR $\left(400 \mathrm{MHz}, \mathrm{CDCl}_{3}\right) \delta 7.58(\mathrm{~d}, J=8.0 \mathrm{~Hz}, 1 \mathrm{H}), 7.34(\mathrm{t}, J=8.0 \mathrm{~Hz}$, 2H), 7.24-6.89 (m, 13H), $6.76(\mathrm{t}, J=8.0 \mathrm{~Hz}, 2 \mathrm{H}), 6.61(\mathrm{~d}, J=4.0 \mathrm{~Hz}, 2 \mathrm{H}), 6.49(\mathrm{br}, 1 \mathrm{H}), 6.33(\mathrm{~d}$, $J=8.0 \mathrm{~Hz}, 2 \mathrm{H}), 6.28(\mathrm{~s}, 1 \mathrm{H}), 5.89(\mathrm{~d}, J=8.0 \mathrm{~Hz}, 1 \mathrm{H}), 5.66(\mathrm{~s}, 1 \mathrm{H}), 5.22(\mathrm{~d}, J=8.0 \mathrm{~Hz}, 1 \mathrm{H}), 3.20$ $(\mathrm{d}, J=8.0 \mathrm{~Hz}, 1 \mathrm{H}), 2.14-1.95(\mathrm{~m}, 3 \mathrm{H}), 1.49(\mathrm{~d}, J=16.0 \mathrm{~Hz}, 1 \mathrm{H}), 0.74(\mathrm{~s}, 3 \mathrm{H}), 0.44(\mathrm{~s}, 3 \mathrm{H}) ;{ }^{13} \mathrm{C}$ NMR (100 MHz, $\left.\mathrm{CDCl}_{3}\right) \delta 192.2,154.1,148.2,145.2,141.8,138.4,131.3,131.2,130.7,129.4$, 
$129.3,129.1,128.8,128.8,128.5,127.5,127.4,126.9,126.8,126.2,126.0,125.1,125.1,123.7$, 121.7, 121.1, 117.9, 117.0, 111.2, 106.0, 71.9, 63.2, 52.5, 49.5, 42.2, 41.4, 33.7, 30.0, 26.0. IR $(\mathrm{KBr}) v 3441,2948,1563,1489,1230,759 \mathrm{~cm}^{-1}$. HRMS (ESI) calcd for $\mathrm{C}_{44} \mathrm{H}_{38} \mathrm{ClN}_{3} \mathrm{NaO}$ $[\mathrm{M}+\mathrm{Na}]^{+} 682.2596$, found 682.2587 .

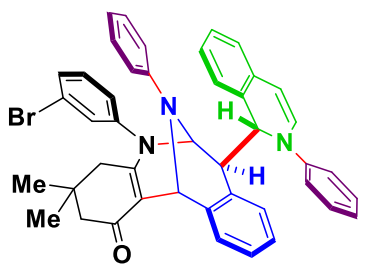

5-(3-bromophenyl)-3,3-dimethyl-13-phenyl-7-(2-phenyl-1,2-dihydroisoquinolin-1-yl)-3,4,5,6,7,12 -hexahydro-6,12-epiminodibenzo[b,e]azocin-1(2H)-one (9i)

White solid obtained by filtration of the precipitate; $92.1 \mathrm{mg}, 87 \%$ yield; $\mathrm{dr}>20: 1 ;$ reaction time $=$ $6 \mathrm{~h}$; mp 221.4-222.3 ${ }^{\circ} \mathrm{C} ;{ }^{1} \mathrm{H}$ NMR $\left(400 \mathrm{MHz}, \mathrm{CDCl}_{3}\right) \delta 7.57(\mathrm{~d}, J=8.0 \mathrm{~Hz}, 1 \mathrm{H}), 7.37-7.30(\mathrm{~m}$, 3H), 7.22-7.12 (m, 4H), 7.06-6.97 (m, 7H), $6.91(\mathrm{t}, J=8.0 \mathrm{~Hz}, 1 \mathrm{H}), 6.79-6.74(\mathrm{~m}, 3 \mathrm{H}), 6.61(\mathrm{~d}, J$ $=8.0 \mathrm{~Hz}, 1 \mathrm{H}), 6.53(\mathrm{br}, 1 \mathrm{H}), 6.34(\mathrm{~d}, J=8.0 \mathrm{~Hz}, 2 \mathrm{H}), 6.28(\mathrm{~s}, 1 \mathrm{H}), 5.89(\mathrm{~d}, J=4.0 \mathrm{~Hz}, 1 \mathrm{H}), 5.65$ (s, 1H), $5.22(\mathrm{~d}, J=8.0 \mathrm{~Hz}, 1 \mathrm{H}), 3.20(\mathrm{~d}, J=8.0 \mathrm{~Hz}, 1 \mathrm{H}), 2.13-1.95(\mathrm{~m}, 3 \mathrm{H}), 1.49(\mathrm{~d}, J=16.0 \mathrm{~Hz}$, 1H), 0.75 (s, 3H), 0.44 (s, 3H); ${ }^{13} \mathrm{C}$ NMR (100 MHz, $\left.\mathrm{CDCl}_{3}\right) \delta$ 192.3, 154.1, 148.2, 145.2, 142.0, 138.4, 130.7, 130.3, 129.8, 129.7, 129.4, 129.2, 128.8, 128.6, 128.0, 127.5, 127.4, 126.9, 126.6, $126.2,126.0,125.1,123.8,123.7,121.7,121.2,117.9,117.0,111.2,106.0,72.0,63.2,52.5,49.5$, 42.3, 41.4, 33.8, 30.0, 26.0. IR (KBr) v 3451, 2954, 1605, 1561, 1489, 1387, $760 \mathrm{~cm}^{-1}$. HRMS (ESI) calcd for $\mathrm{C}_{44} \mathrm{H}_{38} \mathrm{BrN}_{3} \mathrm{NaO}[\mathrm{M}+\mathrm{Na}]^{+}$726.2090, found 726.2098.

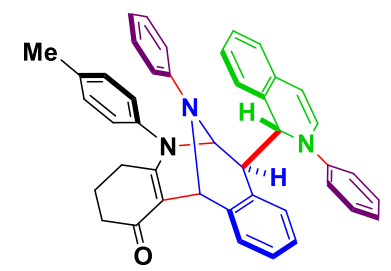

13-phenyl-7-(2-phenyl-1,2-dihydroisoquinolin-1-yl)-5-( $p$-tolyl)-3,4,5,6,7,12-hexahydro-6,12-epim inodibenzo[b,e $]$ azocin-1(2H)-one $(\mathbf{9 j})$

White solid obtained by filtration of the precipitate; $80.9 \mathrm{mg}, 88 \%$ yield; $\mathrm{dr}>20: 1$; reaction time $=$ $6 \mathrm{~h} ; \mathrm{mp} 238.3-239.1{ }^{\circ} \mathrm{C} ;{ }^{1} \mathrm{H}$ NMR $\left(400 \mathrm{MHz}, \mathrm{CDCl}_{3}\right) \delta 7.61(\mathrm{~d}, J=8.0 \mathrm{~Hz}, 1 \mathrm{H}), 7.35(\mathrm{t}, J=8.0$ $\mathrm{Hz}, 2 \mathrm{H}), 7.17(\mathrm{t}, J=8.0 \mathrm{~Hz}, 2 \mathrm{H}), 7.15-7.09(\mathrm{~m}, 2 \mathrm{H}), 7.02-6.89(\mathrm{~m}, 8 \mathrm{H}), 6.77(\mathrm{t}, J=8.0 \mathrm{~Hz}, 1 \mathrm{H})$, $6.68(\mathrm{~d}, J=8.0 \mathrm{~Hz}, 1 \mathrm{H}), 6.61-6.56(\mathrm{~m}, 3 \mathrm{H}), 6.36(\mathrm{~d}, J=8.0 \mathrm{~Hz}, 2 \mathrm{H}), 6.23(\mathrm{~s}, 1 \mathrm{H}), 5.84(\mathrm{~d}, J=8.0$ $\mathrm{Hz}, 1 \mathrm{H}), 5.60(\mathrm{~s}, 1 \mathrm{H}), 5.24(\mathrm{~d}, J=8.0 \mathrm{~Hz}, 1 \mathrm{H}), 3.30(\mathrm{~d}, J=12.0 \mathrm{~Hz}, 1 \mathrm{H}), 2.32(\mathrm{~s}, 3 \mathrm{H}), 2.19-2.05$ 
(m, 3H), 1.78-1.51 (m, 4H); ${ }^{13} \mathrm{C}$ NMR (100 MHz, $\left.\mathrm{CDCl}_{3}\right) \delta 192.2,157.1,148.5,145.3,139.0$, 137.9, 137.0, 131.3, 130.5, 130.0, 129.6, 129.2, 129.0, 128.6, 127.6, 127.4, 127.2, 126.9, 126.2, 126.0, 125.0, 123.6, 121.4, 121.1, 118.1, 117.1, 110.2, 105.8, 72.2, 63.3, 52.3, 42.9, 36.0, 27.4, 22.2, 21.0. IR (KBr) v 3448, 2936, 1607, 1556, $1500,758 \mathrm{~cm}^{-1}$. HRMS (ESI) calcd for $\mathrm{C}_{43} \mathrm{H}_{38} \mathrm{~N}_{3} \mathrm{O}$ $[\mathrm{M}+\mathrm{H}]^{+}$612.3009, found 612.3013.

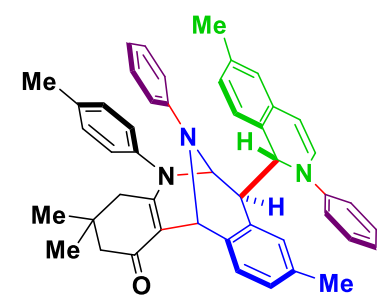

3,3,9-trimethyl-7-(6-methyl-2-phenyl-1,2-dihydroisoquinolin-1-yl)-13-phenyl-5-( $p$-tolyl)-3,4,5,6,7, 12-hexahydro-6,12-epiminodibenzo[b,e]azocin-1(2H)-one (9k)

White solid obtained by filtration of the precipitate; $72.8 \mathrm{mg}, 73 \%$ yield; $\mathrm{dr}>20: 1$; reaction time $=$ $6 \mathrm{~h} ; \mathrm{mp} 232.8-233.4{ }^{\circ} \mathrm{C} ;{ }^{1} \mathrm{H}$ NMR $\left(400 \mathrm{MHz}, \mathrm{CDCl}_{3}\right) \delta 7.39(\mathrm{~d}, J=8.0 \mathrm{~Hz}, 1 \mathrm{H}), 7.25(\mathrm{t}, J=8.0$ $\mathrm{Hz}, 2 \mathrm{H}), 7.11(\mathrm{~d}, J=8.0 \mathrm{~Hz}, 2 \mathrm{H}), 6.91-6.84(\mathrm{~m}, 6 \mathrm{H}), 6.75(\mathrm{~d}, J=8.0 \mathrm{~Hz}, 2 \mathrm{H}), 6.67(\mathrm{t}, J=8.0 \mathrm{~Hz}$, 2H), $6.53(\mathrm{t}, J=8.0 \mathrm{~Hz}, 4 \mathrm{H}), 6.28(\mathrm{~d}, J=4.0 \mathrm{~Hz}, 2 \mathrm{H}), 6.12(\mathrm{~s}, 1 \mathrm{H}), 5.69(\mathrm{~d}, J=4.0 \mathrm{~Hz}, 1 \mathrm{H}), 5.59$ (s, 1H), $5.12(\mathrm{~d}, J=8.0 \mathrm{~Hz}, 1 \mathrm{H}), 3.14(\mathrm{~d}, J=8.0 \mathrm{~Hz}, 1 \mathrm{H}), 2.24(\mathrm{~d}, J=16.0 \mathrm{~Hz}, 6 \mathrm{H}), 1.98-1.85(\mathrm{~m}$, $6 \mathrm{H}), 1.43(\mathrm{~d}, J=16.0 \mathrm{~Hz}, 1 \mathrm{H}), 0.63(\mathrm{~s}, 3 \mathrm{H}), 0.38(\mathrm{~s}, 3 \mathrm{H}) ;{ }^{13} \mathrm{C} \mathrm{NMR}\left(100 \mathrm{MHz}, \mathrm{CDCl}_{3}\right) \delta 191.7$, 155.1, 148.5, 145.3, 138.0, 136.8, 126.7, 136.0, 135.3, 131.3, 131.1, 129.9, 129.3, 129.2, 129.0, $128.5,127.8,126.7,125.8,125.7,125.3,124.1,121.2,120.8,117.9,116.7,109.8,105.6,72.1$, 62.8, 52.0, 49.5, 42.7, 41.2, 33.5, 30.0, 26.2, 21.1, 21.0, 20.9. IR (KBr) v 3434, 2956, 1607, 1554, $750 \mathrm{~cm}^{-1}$. HRMS (ESI) calcd for $\mathrm{C}_{47} \mathrm{H}_{45} \mathrm{~N}_{3} \mathrm{NaO}[\mathrm{M}+\mathrm{Na}]^{+} 690.3455$, found 690.3454 .

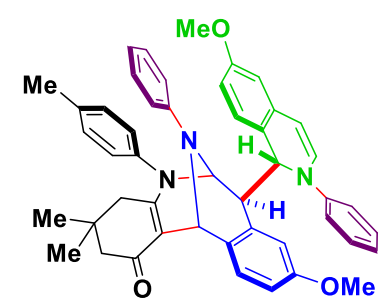

9-methoxy-7-(6-methoxy-2-phenyl-1,2-dihydroisoquinolin-1-yl)-3,3-dimethyl-13-phenyl-5-( $p$-tol yl)-3,4,5,6,7,12-hexahydro-6,12-epiminodibenzo[b,e]azocin-1(2H)-one (9l)

White solid obtained by filtration of the precipitate; $75.5 \mathrm{mg}, 72 \%$ yield; $\mathrm{dr}>20: 1$; reaction time $=$ $6 \mathrm{~h} ; \mathrm{mp} 241.2-241.9{ }^{\circ} \mathrm{C} ;{ }^{1} \mathrm{H}$ NMR $\left(400 \mathrm{MHz}, \mathrm{CDCl}_{3}\right) \delta 7.50(\mathrm{~d}, J=8.0 \mathrm{~Hz}, 1 \mathrm{H}), 7.31(\mathrm{t}, J=8.0$ $\mathrm{Hz}, 2 \mathrm{H}), 7.19(\mathrm{~d}, J=8.0 \mathrm{~Hz}, 2 \mathrm{H}), 7.02-6.95(\mathrm{~m}, 5 \mathrm{H}), 6.71(\mathrm{t}, J=8.0 \mathrm{~Hz}, 1 \mathrm{H}), 6.67-6.59(\mathrm{~m}, 7 \mathrm{H})$, 
$6.45(\mathrm{~d}, J=4.0 \mathrm{~Hz}, 1 \mathrm{H}), 6.37(\mathrm{~d}, J=8.0 \mathrm{~Hz}, 2 \mathrm{H}), 6.20(\mathrm{~s}, 1 \mathrm{H}), 5.79(\mathrm{~d}, J=8.0 \mathrm{~Hz}, 1 \mathrm{H}), 5.63(\mathrm{~s}$, 1H), $5.21(\mathrm{~d}, J=8.0 \mathrm{~Hz}, 1 \mathrm{H}), 3.78(\mathrm{~s}, 3 \mathrm{H}), 3.45(\mathrm{~s}, 3 \mathrm{H}), 3.23(\mathrm{~d}, J=8.0 \mathrm{~Hz}, 1 \mathrm{H}), 2.34(\mathrm{~s}, 3 \mathrm{H})$, 2.06-1.97 (m, 3H), $1.52(\mathrm{~d}, J=16.0 \mathrm{~Hz}, 1 \mathrm{H}), 0.71(\mathrm{~s}, 3 \mathrm{H}), 0.47(\mathrm{~s}, 3 \mathrm{H}) ;{ }^{13} \mathrm{C}$ NMR $(100 \mathrm{MHz}$, $\left.\mathrm{CDCl}_{3}\right) \delta 191.7,159.1,157.7,155.1,148.4,145.1,137.9,136.8,132.5,131.7,130.9,130.0,129.2$, 129.1, 128.6, 127.8, 127.0, 121.3, 121.1, 120.7, 117.8, 116.7, 115.7, 113.7, 111.2, 109.8, 108.1, 105.7, 71.9, 62.6, 55.3, 55.3, 51.7, 49.5, 42.9, 41.1, 33.4, 29.7, 26.3, 21.0. IR (KBr) v 3449, 2942, $1605,1561,1498,1235,754 \mathrm{~cm}^{-1}$. HRMS (ESI) calcd for $\mathrm{C}_{47} \mathrm{H}_{45} \mathrm{~N}_{3} \mathrm{NaO}_{3}[\mathrm{M}+\mathrm{Na}]^{+} 722.3353$, found 722.3343 .

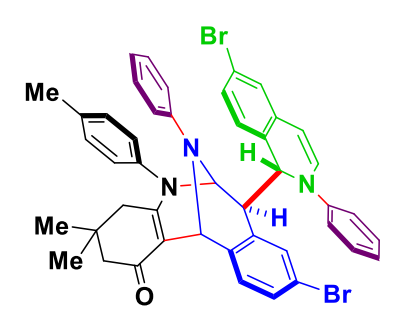

9-bromo-7-(6-bromo-2-phenyl-1,2-dihydroisoquinolin-1-yl)-3,3-dimethyl-13-phenyl-5-( -tolyl)-3, 4,5,6,7,12-hexahydro-6,12-epiminodibenzo[b,e]azocin-1(2H)-one (9m)

White solid obtained by filtration of the precipitate; $92.8 \mathrm{mg}, 78 \%$ yield; $\mathrm{dr}>20: 1$; reaction time $=$ 6 h; mp 246.7-247.2 ${ }^{\circ} \mathrm{C} ;{ }^{1} \mathrm{H}$ NMR $\left(400 \mathrm{MHz}, \mathrm{CDCl}_{3}\right) \delta 7.47(\mathrm{~d}, J=8.0 \mathrm{~Hz}, 1 \mathrm{H}), 7.36(\mathrm{t}, J=8.0$ $\mathrm{Hz}, 2 \mathrm{H}), 7.24(\mathrm{~d}, J=8.0 \mathrm{~Hz}, 1 \mathrm{H}), 7.14\left(\mathrm{dd}, J_{I}=J_{2}=8.0 \mathrm{~Hz}, 4 \mathrm{H}\right), 7.07-6.99(\mathrm{~m}, 6 \mathrm{H}), 6.84(\mathrm{t}, J=$ $8.0 \mathrm{~Hz}, 1 \mathrm{H}), 6.69$ (d, $J=8.0 \mathrm{~Hz}, 2 \mathrm{H}), 6.57(\mathrm{~d}, J=8.0 \mathrm{~Hz}, 2 \mathrm{H}), 6.41(\mathrm{~d}, J=8.0 \mathrm{~Hz}, 2 \mathrm{H}), 6.15(\mathrm{~s}$, 1H), $5.72(\mathrm{~d}, J=4.0 \mathrm{~Hz}, 1 \mathrm{H}), 5.60(\mathrm{~s}, 1 \mathrm{H}), 5.19(\mathrm{~d}, J=8.0 \mathrm{~Hz}, 1 \mathrm{H}), 3.18(\mathrm{~d}, J=8.0 \mathrm{~Hz}, 1 \mathrm{H}), 2.37$ (s, 3H), 2.06-1.94 (m, 3H), $1.56(\mathrm{~d}, J=16.0 \mathrm{~Hz}, 1 \mathrm{H}), 0.73(\mathrm{~s}, 3 \mathrm{H}), 0.51(\mathrm{~s}, 3 \mathrm{H}) ;{ }^{13} \mathrm{C}$ NMR (100 $\left.\mathrm{MHz}, \mathrm{CDCl}_{3}\right) \delta 191.7,155.3,148.1,144.7,138.1,137.5,137.4,133.5,133.3,133.3,131.6,130.4$, $130.3,130.1,129.4,128.8,128.0,127.8,127.5,126.1,125.9,121.9,121.4,119.6,118.1,117.1$, 108.8, 104.1, 71.7, 62.7, 52.2, 49.4, 42.8, 41.1, 33.4, 29.7, 26.4, 21.0. IR (KBr) v 3449, 2955, 2230, 1553, 1499, $724 \mathrm{~cm}^{-1}$. HRMS (ESI) calcd for $\mathrm{C}_{45} \mathrm{H}_{39} \mathrm{Br}_{2} \mathrm{~N}_{3} \mathrm{NaO}[\mathrm{M}+\mathrm{Na}]^{+} 818.1352$, found 818.1356.

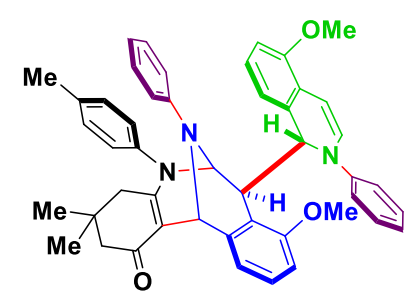


8-methoxy-7-(5-methoxy-2-phenyl-1,2-dihydroisoquinolin-1-yl)-3,3-dimethyl-13-phenyl-5-( $p$-tol yl)-3,4,5,6,7,12-hexahydro-6,12-epiminodibenzo[b,e]azocin-1(2H)-one (9n)

White solid obtained by filtration of the precipitate; $80.2 \mathrm{mg}, 76 \%$ yield; $\mathrm{dr}>20: 1$; reaction time $=$ $6 \mathrm{~h} ; \mathrm{mp} 181.2-181 .{ }^{\circ} \mathrm{C} ;{ }^{1} \mathrm{H}$ NMR (400 MHz, $\left.\mathrm{CDCl}_{3}\right) \delta$ 7.22-7.11 (m, 7H), 7.06 (br, 2H), 6.88 (dd, $\left.J_{I}=J_{2}=8.0 \mathrm{~Hz}, 7 \mathrm{H}\right), 6.73(\mathrm{~d}, J=8.0 \mathrm{~Hz}, 1 \mathrm{H}), 6.60(\mathrm{t}, J=8.0 \mathrm{~Hz}, 1 \mathrm{H}), 6.56(\mathrm{~d}, J=8.0 \mathrm{~Hz}, 1 \mathrm{H})$, $6.40(\mathrm{~d}, J=8.0 \mathrm{~Hz}, 1 \mathrm{H}), 6.20(\mathrm{~s}, 1 \mathrm{H}), 5.78(\mathrm{~d}, J=4.0 \mathrm{~Hz}, 1 \mathrm{H}), 5.66(\mathrm{~d}, J=8.0 \mathrm{~Hz}, 2 \mathrm{H}), 5.48(\mathrm{~d}, J$ $=8.0 \mathrm{~Hz}, 1 \mathrm{H}), 3.84(\mathrm{~s}, 3 \mathrm{H}), 3.71(\mathrm{~d}, J=4.0 \mathrm{~Hz}, 1 \mathrm{H}), 3.50(\mathrm{~s}, 3 \mathrm{H}), 2.31(\mathrm{~s}, 3 \mathrm{H}), 1.98\left(\mathrm{dd}, J_{l}=J_{2}=\right.$ $4.0 \mathrm{~Hz}, 2 \mathrm{H}), 1.89(\mathrm{~d}, J=16.0 \mathrm{~Hz}, 1 \mathrm{H}), 1.54(\mathrm{~d}, J=16.0 \mathrm{~Hz}, 1 \mathrm{H}), 0.68(\mathrm{~s}, 3 \mathrm{H}), 0.50(\mathrm{~s}, 3 \mathrm{H}) ;{ }^{13} \mathrm{C}$ NMR $\left(100 \mathrm{MHz}, \mathrm{CDCl}_{3}\right) \delta 191.5,157.6,155.2,152.6,147.9,145.7,141.6,138.6,137.1,130.2$, $129.3,128.8,128.6,128.4,127.9,125.0,121.3,120.9,120.6,119.2,119.1,118.6,118.3,117.8$, 108.9, 108.8, 107.7, 98.1, 72.1, 60.4, 55.2, 55.1, 51.5, 49.5, 44.4, 41.1, 33.3, 29.7, 26.5, 21.0. IR $(\mathrm{KBr}) v 3441,2950,1683,1558,1502,1264,765 \mathrm{~cm}^{-1}$. HRMS (ESI) calcd for $\mathrm{C}_{47} \mathrm{H}_{45} \mathrm{~N}_{3} \mathrm{NaO}_{3}$ $[\mathrm{M}+\mathrm{Na}]^{+} 722.3353$, found 722.3360 .

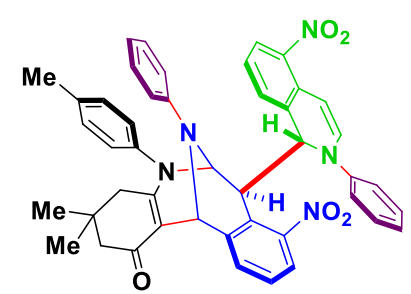

3,3-dimethyl-8-nitro-7-(5-nitro-2-phenyl-1,2-dihydroisoquinolin-1-yl)-13-phenyl-5-( $p$-tolyl)-3,4,5, 6,7,12-hexahydro-6,12-epiminodibenzo[b,e $]$ azocin-1(2H)-one $(\mathbf{9 o})$

White solid obtained by filtration of the precipitate; $93.4 \mathrm{mg}, 85 \%$ yield; $\mathrm{dr}>20: 1$; reaction time $=$ $6 \mathrm{~h} ; \mathrm{mp} 237.9-238.4{ }^{\circ} \mathrm{C} ;{ }^{1} \mathrm{H}$ NMR $\left(400 \mathrm{MHz}, \mathrm{CDCl}_{3}\right) \delta 7.76(\mathrm{~d}, J=8.0 \mathrm{~Hz}, 1 \mathrm{H}), 7.67(\mathrm{~d}, J=8.0$ $\mathrm{Hz}, 1 \mathrm{H}), 7.37(\mathrm{~d}, J=8.0 \mathrm{~Hz}, 1 \mathrm{H}), 7.25(\mathrm{t}, J=8.0 \mathrm{~Hz}, 2 \mathrm{H}), 7.08(\mathrm{t}, J=8.0 \mathrm{~Hz}, 3 \mathrm{H}), 7.01(\mathrm{~d}, J=$ $8.0 \mathrm{~Hz}, 2 \mathrm{H}), 6.93(\mathrm{q}, J=8.0 \mathrm{~Hz}, 3 \mathrm{H}), 6.81(\mathrm{t}, J=8.0 \mathrm{~Hz}, 3 \mathrm{H}), 6.78-6.68(\mathrm{~m}, 3 \mathrm{H}), 6.51(\mathrm{t}, J=8.0$ $\mathrm{Hz}, 2 \mathrm{H}), 6.34(\mathrm{~d}, J=8.0 \mathrm{~Hz}, 1 \mathrm{H}), 6.17(\mathrm{~s}, 1 \mathrm{H}), 5.77(\mathrm{~s}, 1 \mathrm{H}), 5.58(\mathrm{~d}, J=4.0 \mathrm{~Hz}, 1 \mathrm{H}), 4.44\left(\mathrm{dd}, J_{I}\right.$ $\left.=J_{2}=4.0 \mathrm{~Hz}, 1 \mathrm{H}\right), 2.35(\mathrm{~s}, 3 \mathrm{H}), 1.90(\mathrm{~s}, 2 \mathrm{H}), 1.86(\mathrm{~d}, J=16.0 \mathrm{~Hz}, 1 \mathrm{H}), 1.47(\mathrm{~d}, J=16.0 \mathrm{~Hz}, 1 \mathrm{H})$, 0.59 (s, 3H), $0.42(\mathrm{~s}, 3 \mathrm{H}) ;{ }^{13} \mathrm{C} \mathrm{NMR}\left(100 \mathrm{MHz}, \mathrm{CDCl}_{3}\right) \delta 191.7,156.0,150.5,147.2,145.3,142.4$, $141.5,138.1,127.4,126.3,131.8,129.5,129.4,129.1,129.0,128.1,128.0,126.0,124.9,124.8$, 124.3, 123.7, 123.1, 121.9, 120.3, 117.7, 107.7, 97.7, 72.5, 63.3, 51.6, 49.3, 45.6, 40.7, 33.2, 29.4, 26.5, 21.2. IR (KBr) $v$ 3444, 2950, 1560, 1514, 1266, $752 \mathrm{~cm}^{-1}$. HRMS (ESI) calcd for $\mathrm{C}_{45} \mathrm{H}_{39} \mathrm{~N}_{5} \mathrm{NaO}_{5}[\mathrm{M}+\mathrm{Na}]^{+}$752.2843, found 752.2856. 


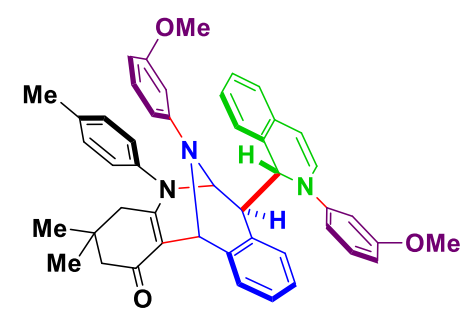

13-(3-methoxyphenyl)-7-(2-(3-methoxyphenyl)-1,2-dihydroisoquinolin-1-yl)-3,3-dimethyl-5-(p-to lyl)-3,4,5,6,7,12-hexahydro-6,12-epiminodibenzo[b,e]azocin-1(2H)-one (9p)

White solid obtained by filtration of the precipitate; $79.7 \mathrm{mg}, 76 \%$ yield; $\mathrm{dr}>20: 1$; reaction time $=$ $6 \mathrm{~h} ; \mathrm{mp} 237.3-238.1{ }^{\circ} \mathrm{C} ;{ }^{1} \mathrm{H}$ NMR $\left(400 \mathrm{MHz}, \mathrm{CDCl}_{3}\right) \delta 7.50(\mathrm{~d}, J=8.0 \mathrm{~Hz}, 1 \mathrm{H}), 7.16(\mathrm{q}, J=8.0$ $\mathrm{Hz}, 1 \mathrm{H}), 7.11-7.04(\mathrm{~m}, 2 \mathrm{H}), 6.98(\mathrm{q}, J=8.0 \mathrm{~Hz}, 2 \mathrm{H}), 6.87(\mathrm{t}, J=8.0 \mathrm{~Hz}, 3 \mathrm{H}), 6.82(\mathrm{t}, J=8.0 \mathrm{~Hz}$, $1 \mathrm{H}), 6.73(\mathrm{t}, J=8.0 \mathrm{~Hz}, 2 \mathrm{H}), 6.68(\mathrm{~s}, 1 \mathrm{H}), 6.52-6.46(\mathrm{~m}, 4 \mathrm{H}), 6.25\left(\mathrm{dd}, J_{I}=J_{2}=4.0 \mathrm{~Hz}, 1 \mathrm{H}\right), 6.17$ (s, 1H), $5.92(\mathrm{~d}, J=8.0 \mathrm{~Hz}, 1 \mathrm{H}), 5.78(\mathrm{t}, J=8.0 \mathrm{~Hz}, 2 \mathrm{H}), 5.53(\mathrm{~s}, 1 \mathrm{H}), 5.12(\mathrm{~d}, J=8.0 \mathrm{~Hz}, 1 \mathrm{H})$, $3.75(\mathrm{~s}, 3 \mathrm{H}), 3.50(\mathrm{~s}, 3 \mathrm{H}), 3.15(\mathrm{~d}, J=4.0 \mathrm{~Hz}, 1 \mathrm{H}), 2.26(\mathrm{~s}, 3 \mathrm{H}), 1.99-1.86(\mathrm{~m}, 4 \mathrm{H}), 1.46(\mathrm{~d}, J=$ $16.0 \mathrm{~Hz}, 1 \mathrm{H}), 0.63$ (s, 3H), 0.43 (s, 3H); ${ }^{13} \mathrm{C}$ NMR (100 MHz, $\left.\mathrm{CDCl}_{3}\right) \delta 191.6,160.6,159.9,155.3$, $149.9,146.7,138.7,137.7,136.8,131.3,130.7,130.0,129.8,129.4,129.1,128.9,127.8,127.4$, 127.3, 127.0, 126.1, 126.0, 125.1, 123.6, 110.6, 109.8, 109.7, 107.5, 107.1, 106.1, 104.2, 102.2, 72.1, 63.2, 55.3, 55.0, 52.7, 49.5, 42.3, 41.1, 33.5, 29.7, 26.3, 20.9. IR (KBr) v 3420, 2953, 1598, 1558, 1264, $766 \mathrm{~cm}^{-1}$. HRMS (ESI) calcd for $\mathrm{C}_{47} \mathrm{H}_{45} \mathrm{~N}_{3} \mathrm{NaO}_{3}[\mathrm{M}+\mathrm{Na}]^{+} 722.3353$, found 722.3356 .

\section{Experimental data for the scalable preparation of $4 a$}

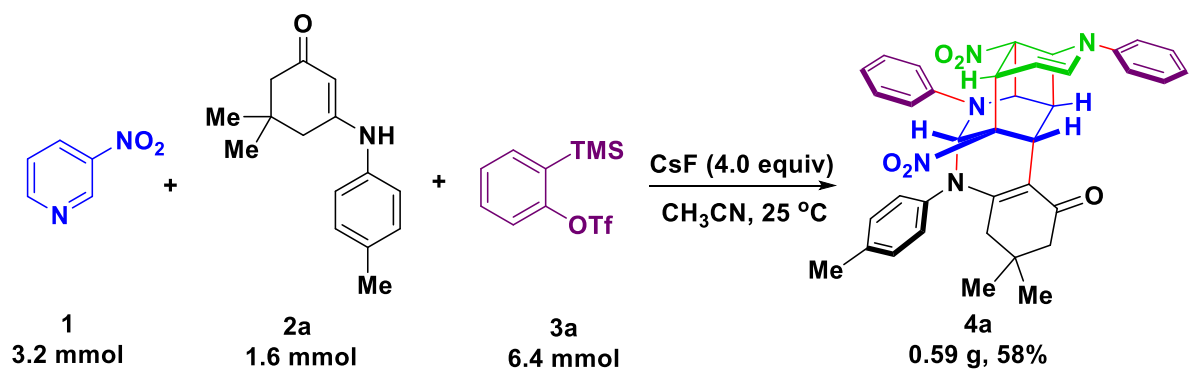

General procedure: To a $25.0 \mathrm{~mL}$ round-bottom flask were successively added 3-nitropyridine $\mathbf{1}$ ( $0.40 \mathrm{~g}, 3.2 \mathrm{mmol})$, enaminone $\mathbf{2 a}(0.37 \mathrm{~g}, 1.6 \mathrm{mmol})$, aryne precursors $3 \mathbf{a}$ (1.6 $\mathrm{mL}, 6.4 \mathrm{mmol}), \mathrm{CsF}(1.00 \mathrm{~g}, 6.4 \mathrm{mmol})$ and $5.0 \mathrm{~mL}$ of $\mathrm{CH}_{3} \mathrm{CN}$. The resulting mixture was stirred at $25{ }^{\circ} \mathrm{C}$ for $48 \mathrm{~h}$. During the reaction process, a large amount of precipitate occurred, and only a simple filtration was required to purify them once the reaction went completion. The desired product 4a was obtained as yellow solid in $58 \%$ yield $(0.59 \mathrm{~g})$.

\section{Experimental data for derivations of $\mathbf{4 g}$}




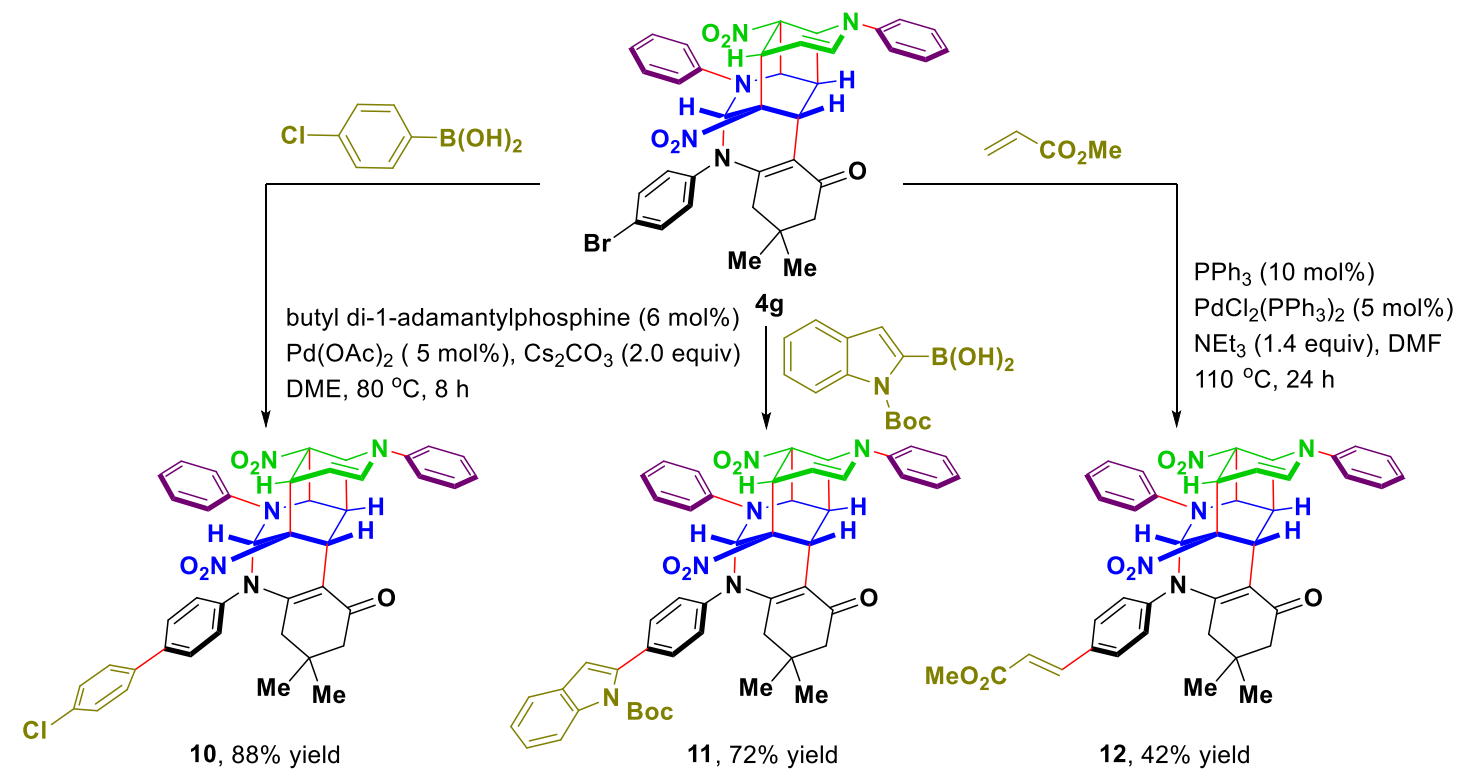

General procedure for the formation of 10: Under nitrogen atmosphere, compound $\mathbf{4 g}$ (111.1 mg, 0.16mmol), 4-chlorophenyl boronic acid (37.5 mg, 0.24mmol, 1.5 equiv), $\mathrm{Cs}_{2} \mathrm{CO}_{3}$ (104.3 mg, $0.32 \mathrm{mmol}, 2.0$ equiv), $\mathrm{Pd}(\mathrm{OAc})_{2}$ (0.05 equiv) and butyl di-1-adamantylphosphine (0.06 equiv) were successively added to a $15 \mathrm{~mL}$ dried tube, followed by addition of $2.0 \mathrm{~mL}$ DME. The resulting mixture was stirred at $80{ }^{\circ} \mathrm{C}$ in oil bath for $8 \mathrm{~h}$, and then the reaction mixture was directly subjected to silica gel column chromatography (petroleum ether/ ethyl acetate as eluent) to afford the corresponding product $\mathbf{1 0}$ as a yellow solid in $88 \%$ yield.

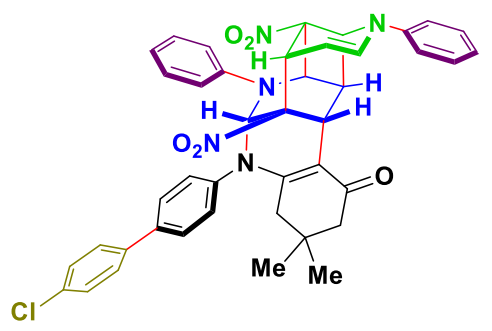

5-(4'-chloro-[1,1'-biphenyl]-4-yl)-3,3-dimethyl-7b,13-dinitro-7,11-diphenyl-3,4,5,6,7,7a,7b,8,11,1 1a,11b,12-dodecahydro-6,8,12-(epimethanetriyl)benzo[ $d]$ pyrido[3',2':3,4]cyclobuta[1,2-g][1,3]dia zocin-1(2H)-one (10)

Yellow solid obtained by column chromatography (petroleum ether/ethyl acetate $=10: 1$ to $5: 1$ ); $102.6 \mathrm{mg}, 88 \%$ yield; $\mathrm{dr}>20: 1$; reaction time $=8 \mathrm{~h}$; mp 223.7-224.2 ${ }^{\circ} \mathrm{C} ;{ }^{1} \mathrm{H}$ NMR $(400 \mathrm{MHz}$, $\left.\mathrm{CDCl}_{3}\right) \delta 7.43(\mathrm{t}, J=8.0 \mathrm{~Hz}, 3 \mathrm{H}), 7.38(\mathrm{~d}, J=8.0 \mathrm{~Hz}, 4 \mathrm{H}), 7.23\left(\mathrm{dd}, J_{l}=J_{2}=8.0 \mathrm{~Hz}, 5 \mathrm{H}\right), 7.10(\mathrm{t}$, $J=8.0 \mathrm{~Hz}, 2 \mathrm{H}), 6.98(\mathrm{t}, J=8.0 \mathrm{~Hz}, 3 \mathrm{H}), 6.63(\mathrm{br}, 2 \mathrm{H}), 6.09(\mathrm{~s}, 1 \mathrm{H}), 4.53\left(\mathrm{dd}, J_{l}=J_{2}=4.0 \mathrm{~Hz}\right.$, $1 \mathrm{H}), 4.35(\mathrm{t}, J=8.0 \mathrm{~Hz}, 1 \mathrm{H}), 4.20(\mathrm{~d}, J=8.0 \mathrm{~Hz}, 1 \mathrm{H}), 4.08\left(\mathrm{dd}, J_{l}=J_{2}=4.0 \mathrm{~Hz}, 1 \mathrm{H}\right), 4.06(\mathrm{~s}, 1 \mathrm{H})$, $3.28(\mathrm{t}, J=8.0 \mathrm{~Hz}, 1 \mathrm{H}), 2.29(\mathrm{t}, J=16.0 \mathrm{~Hz}, 2 \mathrm{H}), 2.04(\mathrm{t}, J=16.0 \mathrm{~Hz}, 2 \mathrm{H}), 0.99(\mathrm{~s}, 3 \mathrm{H}), 0.95(\mathrm{~s}$, 
$3 \mathrm{H}) ;{ }^{13} \mathrm{C}$ NMR (100 MHz, $\left.\mathrm{CDCl}_{3}\right) \delta 194.2,153.0,145.4,145.1,141.6,139.2,138.0,134.0,133.8$, 129.6, 129.6, 129.0, 128.1, 127.9, 127.7, 125.1, 124.0, 123.6, 118.7, 108.2, 87.4, 85.5, 84.4, 59.8, 52.4, 50.1, 48.5, 41.7, 38.6, 33.0, 28.9, 26.9, 25.4. IR (KBr) v 3431, 2954, 1590, 1544, 1490, 1381, $754 \mathrm{~cm}^{-1}$. HRMS (ESI) calcd for $\mathrm{C}_{42} \mathrm{H}_{37} \mathrm{ClN}_{5} \mathrm{O}_{5}[\mathrm{M}+\mathrm{H}]^{+} 726.2478$, found 726.2481 .

General procedure for the formation of 11: Under nitrogen atmosphere, compound $\mathbf{4 g}$ (111.1 mg, 0.16mmol), 2-indolylboronic acid (62.7 mg, 0.24mmol, 1.5 equiv), $\mathrm{Cs}_{2} \mathrm{CO}_{3}$ (104.3 mg, $0.32 \mathrm{mmol}, 2.0$ equiv), $\mathrm{Pd}(\mathrm{OAc})_{2}$ (0.05 equiv) and butyl di-1-adamantylphosphine (0.06 equiv) were successively added to a $15 \mathrm{~mL}$ dried tube, followed by addition of $2.0 \mathrm{~mL}$ DME. The resulting mixture was stirred at $80^{\circ} \mathrm{C}$ in oil bath for $8 \mathrm{~h}$, and then the reaction mixture was directly subjected to silica gel column chromatography (petroleum ether/ ethyl acetate as eluent) to afford the corresponding product $\mathbf{1 1}$ as a yellow liquid in $\mathbf{7 2} \%$ yield.

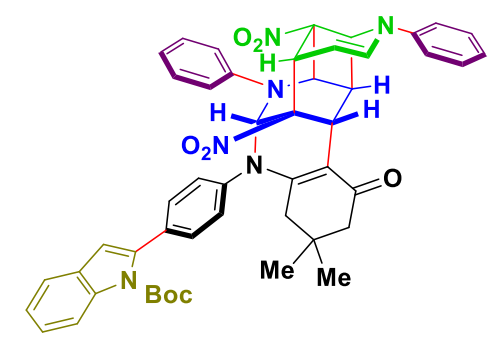

tert-butyl 2-(4-(3,3-dimethyl-7b,13-dinitro-1-oxo-7,11-diphenyl-2,3,4,6,7,7a,7b,8,11,11a,11b,12dodecahydro-6,8,12-(epimethanetriyl)benzo[ $d]$ pyrido[3',2':3,4]cyclobuta[1,2-g][1,3]diazocin-5(1 $H$ )-yl)phenyl)-1 $H$-indole-1-carboxylate (11)

Yellow liquid obtained by column chromatography (petroleum ether/ethyl acetate $=7: 1$ to $3: 1$ ); $95.8 \mathrm{mg}, 72 \%$ yield; $\mathrm{dr}>20: 1$; reaction time $=8 \mathrm{~h} ;{ }^{1} \mathrm{H}$ NMR $\left(400 \mathrm{MHz}, \mathrm{CDCl}_{3}\right) \delta 8.21(\mathrm{~d}, J=8.0$ $\mathrm{Hz}, 1 \mathrm{H}), 7.54(\mathrm{~d}, J=8.0 \mathrm{~Hz}, 1 \mathrm{H}), 7.40(\mathrm{t}, J=8.0 \mathrm{~Hz}, 2 \mathrm{H}), 7.34(\mathrm{t}, J=8.0 \mathrm{~Hz}, 1 \mathrm{H}), 7.30-7.20(\mathrm{~m}$, $8 \mathrm{H}), 7.13(\mathrm{t}, J=8.0 \mathrm{~Hz}, 2 \mathrm{H}), 7.02(\mathrm{~d}, J=8.0 \mathrm{~Hz}, 2 \mathrm{H}), 6.99(\mathrm{~d}, J=8.0 \mathrm{~Hz}, 1 \mathrm{H}), 6.52(\mathrm{br}, 2 \mathrm{H}), 6.06$ $(\mathrm{s}, 1 \mathrm{H}), 4.53\left(\mathrm{dd}, J_{l}=J_{2}=4.0 \mathrm{~Hz}, 1 \mathrm{H}\right), 4.37(\mathrm{t}, J=8.0 \mathrm{~Hz}, 1 \mathrm{H}), 4.18(\mathrm{~d}, J=8.0 \mathrm{~Hz}, 1 \mathrm{H}), 4.09(\mathrm{dd}$, $\left.J_{I}=J_{2}=4.0 \mathrm{~Hz}, 1 \mathrm{H}\right), 4.06(\mathrm{~s}, 1 \mathrm{H}), 3.28(\mathrm{t}, J=8.0 \mathrm{~Hz}, 1 \mathrm{H}), 2.30(\mathrm{t}, J=16.0 \mathrm{~Hz}, 2 \mathrm{H}), 2.06(\mathrm{q}, J=$ $16.0 \mathrm{~Hz}, 2 \mathrm{H}), 1.35$ (s, 9H), 0.99 (s, 3H), 0.97 (s, 3H); $\left.{ }^{13} \mathrm{C} \mathrm{NMR} \mathrm{(100} \mathrm{MHz,} \mathrm{CDCl}_{3}\right) \delta$ 194.3, 153.0, $149.9,145.5,145.2,141.5,138.8,137.6,134.5,134.1,129.8,129.7,129.6,128.9,126.9,125.4$, $124.7,124.4,123.7,123.1,120.5,118.8,115.2,110.7,108.3,87.4,85.5,84.5,83.6,60.0,52.5$, 50.1, 48.6, 41.8, 38.7, 33.1, 28.8, 27.7, 27.1, 25.5. IR (KBr) v 3433, 2931, 1734, 1594, 1326, 743 $\mathrm{cm}^{-1}$. HRMS (ESI) calcd for $\mathrm{C}_{49} \mathrm{H}_{47} \mathrm{~N}_{6} \mathrm{O}_{7}[\mathrm{M}+\mathrm{H}]^{+} 831.3501$, found 831.3505 . 
General procedure for the formation of 12: Under nitrogen atmosphere, compound $\mathbf{4 g}$ (69.5 mg, 0.10mmol), methyl acrylate (10.8 uL, 0.12mmol, 1.2equiv), $\mathrm{Et}_{3} \mathrm{~N}$ (1.4equiv), $\mathrm{PdCl}_{2}\left(\mathrm{PPh}_{3}\right)_{2}$ (0.05 equiv) and triphenylphosphine (0.1equiv) were successively added to a $15 \mathrm{~mL}$ dried tube, followed by addition of $2.0 \mathrm{~mL}$ DMF. The resulting mixture was stirred at $110{ }^{\circ} \mathrm{C}$ in oil bath for $24 \mathrm{~h}$, and then the reaction mixture was directly subjected to silica gel column chromatography (petroleum ether/ ethyl acetate as eluent) to afford the corresponding product $\mathbf{1 2}$ as a yellow liquid in $42 \%$ yield.

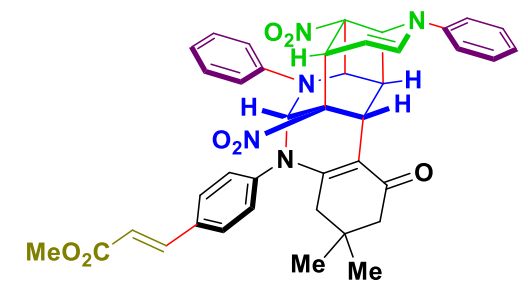

methyl (E)-3-(4-(3,3-dimethyl-7b,13-dinitro-1-oxo-7,11-diphenyl-2,3,4,6,7,7a,7b,8,11,11a,11b,12 -dodecahydro-6,8,12-(epimethanetriyl)benzo[d]pyrido[3',2':3,4]cyclobuta[1,2-g][1,3]diazocin-5(1 $H)$-yl)phenyl)acrylate (12)

Yellow liquid obtained by column chromatography (petroleum ether/ethyl acetate $=10: 1$ to $3: 1$ ); $29.1 \mathrm{mg}, 42 \%$ yield; $\mathrm{dr}>20: 1$; reaction time $=24 \mathrm{~h} ;{ }^{1} \mathrm{H} \mathrm{NMR}\left(400 \mathrm{MHz}, \mathrm{CDCl}_{3}\right) \delta 7.59(\mathrm{~d}, J=$ $16.0 \mathrm{~Hz}, 1 \mathrm{H}), 7.40(\mathrm{t}, J=8.0 \mathrm{~Hz}, 2 \mathrm{H}), 7.31(\mathrm{~d}, J=8.0 \mathrm{~Hz}, 2 \mathrm{H}), 7.23\left(\mathrm{dd}, J_{l}=J_{2}=8.0 \mathrm{~Hz}, 4 \mathrm{H}\right)$, $7.12(\mathrm{q}, J=8.0 \mathrm{~Hz}, 2 \mathrm{H}), 6.98(\mathrm{~d}, J=8.0 \mathrm{~Hz}, 3 \mathrm{H}), 6.58(\mathrm{br}, 2 \mathrm{H}), 6.37$ (d, $J=16.0 \mathrm{~Hz}, 1 \mathrm{H}), 6.04(\mathrm{~s}$, $1 \mathrm{H}), 4.53\left(\mathrm{dd}, J_{I}=J_{2}=4.0 \mathrm{~Hz}, 1 \mathrm{H}\right), 4.35(\mathrm{t}, J=8.0 \mathrm{~Hz}, 1 \mathrm{H}), 4.17(\mathrm{~d}, J=8.0 \mathrm{~Hz}, 1 \mathrm{H}), 4.08\left(\mathrm{dd}, J_{l}\right.$ $\left.=J_{2}=4.0 \mathrm{~Hz}, 1 \mathrm{H}\right), 4.04(\mathrm{~s}, 1 \mathrm{H}), 3.80(\mathrm{~s}, 3 \mathrm{H}), 3.26(\mathrm{t}, J=8.0 \mathrm{~Hz}, 1 \mathrm{H}), 2.29(\mathrm{t}, J=16.0 \mathrm{~Hz}, 2 \mathrm{H})$, 2.02 (t, $J=16.0 \mathrm{~Hz}, 2 \mathrm{H}), 0.99$ (s, 3H), 0.95 (s, 3H); ${ }^{13} \mathrm{C} \mathrm{NMR}\left(100 \mathrm{MHz}, \mathrm{CDCl}_{3}\right) \delta$ 194.4, 167.0, 152.0, 145.4, 145.2, 143.7, 143.1, 134.1, 133.6, 129.8, 129.7, 129.0, 127.7, 125.4, 124.3, 123.7, 118.9, 118.8, 109.0, 87.4, 85.5, 84.5, 60.0, 52.5, 51.8, 50.1, 48.5, 41.8, 38.6, 33.2, 28.8, 27.0, 25.5. IR (KBr) v 34427, 2951, 1594, 1546, 1502, 1175, $745 \mathrm{~cm}^{-1}$. HRMS (ESI) calcd for $\mathrm{C}_{40} \mathrm{H}_{38} \mathrm{~N}_{5} \mathrm{O}_{7}$ $[\mathrm{M}+\mathrm{H}]^{+} 700.2766$, found 700.2766 .

\section{Crystal structures of $4 a, 6 a, 7 a$ and $9 a$}

\subsection{Crystal structure of $4 a$}

Preparation of the single crystals of $\mathbf{4 a}$ : $15.0 \mathrm{mg}$ of pure compound $\mathbf{4 a}$ was dissolved in the combined solvents of $\mathrm{CHCl}_{3}$ and $\mathrm{EtOH}(6 \mathrm{~mL}, \mathrm{v} / \mathrm{v}=1: 1)$ at room 
temperature. The bottle was sealed by a piece of plastic film with several tiny holes, thus allowing slow evaporation of the solvents at room temperature. After about one week, several small particles were observed at the bottom of the bottle. The crystals were chosen and subjected to the single crystal X-ray diffraction analysis for the determination of the structure and relative configuration of 4a. The data were collected by a Rigaku Gemini E at $293.0 \mathrm{~K}$.

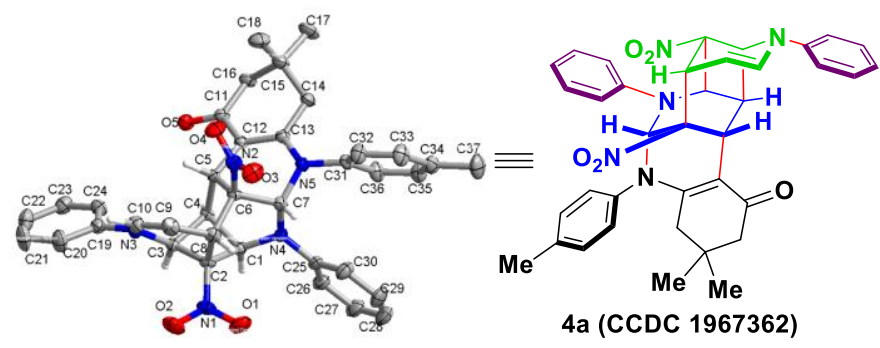

Displacement ellipsoids are drawn at the $30 \%$ probability level.

$\begin{array}{llll}\text { Bond precision: } & \mathrm{C}-\mathrm{C}=0.0074 \mathrm{~A} & \text { Wa } \\ \text { Cell: } & \mathrm{a}=18.48959(17) & \mathrm{b}=13.61717(13) & \mathrm{c}=27.3852(2) \\ & \text { alpha }=90 & \text { beta=90 } & \text { gamma }=90\end{array}$

Temperature: $293 \mathrm{~K}$

$\begin{array}{lll} & \text { Calculated } & \text { Reported } \\ \text { Volume } & 6894.93(10) & 6894.95(11) \\ \text { Space group } & \text { P n a } 21 & \text { P n a 21 } \\ \text { Hall group } & \text { P 2c }-2 \mathrm{n} & \text { P 2c }-2 \mathrm{n} \\ \text { Moiety formula } & 2\left(\mathrm{C}_{37} \mathrm{H}_{35} \mathrm{~N}_{5} \mathrm{O}_{5}\right), \mathrm{CHCl}_{3} & 2\left(\mathrm{C}_{37} \mathrm{H}_{35} \mathrm{~N}_{5} \mathrm{O}_{5}\right), \mathrm{CHCl}_{3} \\ \text { Sum formula } & \mathrm{C}_{75} \mathrm{H}_{71} \mathrm{Cl}_{3} \mathrm{~N}_{10} \mathrm{O}_{10} & \mathrm{C}_{75} \mathrm{H}_{71} \mathrm{Cl}_{3} \mathrm{~N}_{10} \mathrm{O}_{10} \\ \text { Mr } & 1378.77 & 1378.76 \\ \text { Dx,g cm-3 } & 1.328 & 1.328 \\ \text { Z } & 4 & 4 \\ \text { Mu (mm-1) } & 1.757 & 1.757 \\ \text { F000 } & 2888.0 & 2888.0 \\ \text { F000' } & 2900.55 & 22,16,33 \\ \text { h,k,lmax } & 22,16,33 & 11754 \\ \text { Nref } & 13311[6804] & 0.789,1.000 \\ \text { Tmin,Tmax } & 0.744,0.839 & \\ \text { Tmin' } & 0.716 & \\ \text { Correction method= \# Reported T Limits: Tmin=0.789 Tmax=1.000 AbsCorr }= \\ \text { MULTI-SCAN }\end{array}$


$\mathrm{S}=1.019$

Npar $=889$

\subsection{Crystal structure of $6 a$}

Preparation of the single crystals of $\mathbf{6 a}: 15.0 \mathrm{mg}$ of pure compound $\mathbf{6 a}$ was dissolved in the combined solvents of $\mathrm{CH}_{2} \mathrm{Cl}_{2}$ and $\mathrm{EtOH}(6 \mathrm{~mL}, \mathrm{v} / \mathrm{v}=1: 1)$ at room temperature. The bottle was sealed by a piece of plastic film with several tiny holes, thus allowing slow evaporation of the solvents at room temperature. After about one week, several small particles were observed at the bottom of the bottle. The crystals were chosen and subjected to the single crystal X-ray diffraction analysis for the determination of the structure and relative configuration of $6 \mathbf{a}$. The data were collected by a Rigaku Gemini E at 293.0 K.
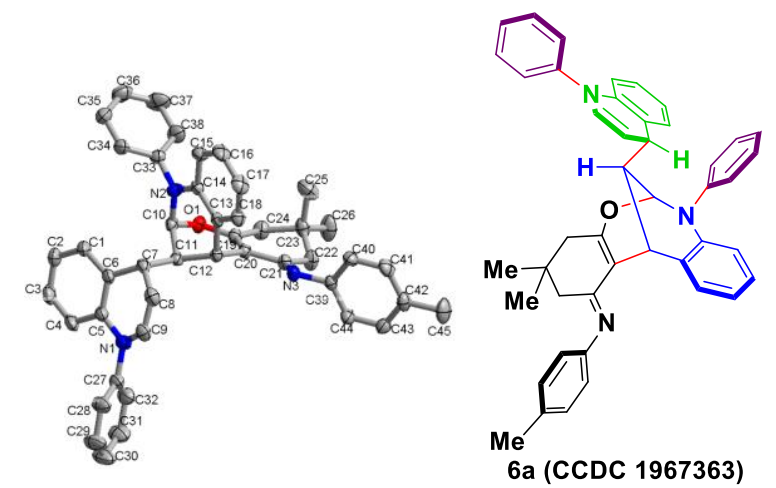

Displacement ellipsoids are drawn at the $30 \%$ probability level.

Bond precision:

$$
\mathrm{C}-\mathrm{C}=0.0033 \mathrm{~A}
$$

Wavelength $=1.54184$

\begin{tabular}{|c|c|c|c|}
\hline Cell: & $a=16.5693(2)$ & $b=10.74988(15)$ & $\mathrm{c}=20.4362(2)$ \\
\hline & alpha $=90$ & beta $=93.2630(11)$ & gamma $=90$ \\
\hline
\end{tabular}

Temperature: 293 K

$\begin{array}{lll} & \text { Calculated } & \text { Reported } \\ \text { Volume } & 3634.15(8) & 3634.17(8) \\ \text { Space group } & \mathrm{P} 21 / \mathrm{n} & \mathrm{P} 121 / \mathrm{n} 1 \\ \text { Hall group } & -\mathrm{P} 2 \mathrm{yn} & -\mathrm{P} 2 \mathrm{yn} \\ \text { Moiety formula } & \mathrm{C}_{45} \mathrm{H}_{41} \mathrm{~N}_{3} \mathrm{O} & \mathrm{C}_{45} \mathrm{H}_{41} \mathrm{~N}_{3} \mathrm{O} \\ \text { Sum formula } & \mathrm{C}_{45} \mathrm{H}_{41} \mathrm{~N}_{3} \mathrm{O} & \mathrm{C}_{45} \mathrm{H}_{41} \mathrm{~N}_{3} \mathrm{O} \\ \text { Mr } & 639.81 & 639.81 \\ \text { Dx,g cm-3 } & 1.169 & 1.169 \\ \text { Z } & 4 & 4 \\ \text { Mu (mm-1) } & 0.539 & 0.539 \\ \text { F000 } & 1360.0 & 1360.0 \\ \text { F000' } & 1363.61 & \\ \text { h,k,lmax } & 19,12,24 & 19,12,24\end{array}$




$\begin{array}{ll}\text { Tmin,Tmax } & 0.919,0.948 \\ \text { Tmin' } & 0.903\end{array}$

Correction method $=$ Not given

Data completeness $=1.000$

$\mathrm{R}($ reflections $)=0.0584(5323)$
$\operatorname{Theta}(\max )=67.073$

$\mathrm{wR} 2($ reflections $)=0.1754(6495)$

$\mathrm{S}=1.036$

Npar $=445$

\subsection{Crystal structure of $7 a$}

Preparation of the single crystals of $7 \mathbf{a}: 10.0 \mathrm{mg}$ of pure compound $\mathbf{7 a}$ was dissolved in the combined solvents of petroleum and ethyl acetate $(6 \mathrm{~mL}, \mathrm{v} / \mathrm{v}=5: 1)$ at $0{ }^{\circ} \mathrm{C}$. The bottle was sealed by a piece of plastic film with one tiny hole, thus allowing slow evaporation of the solvents at $0{ }^{\circ} \mathrm{C}$. After about four weeks, several small particles were observed at the bottom of the bottle. The crystals were chosen and subjected to the single crystal X-ray diffraction analysis for the determination of the structure and relative configuration of 7a. The data were collected by a Bruker D8 VENTURE PHOTON II CCD diffractometer at $273.0 \mathrm{~K}$.

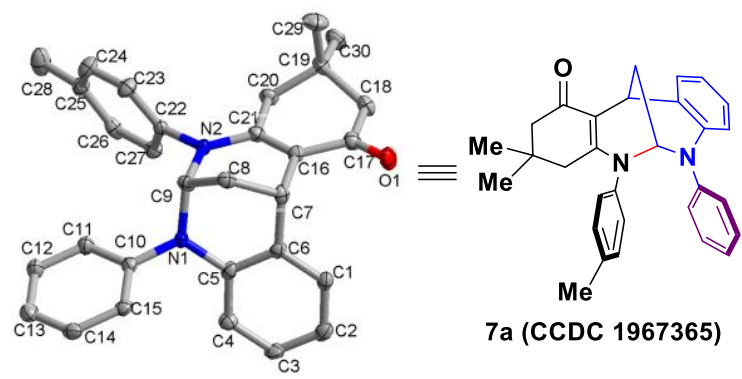

Displacement ellipsoids are drawn at the $30 \%$ probability level.

Bond precision:

$$
\mathrm{C}-\mathrm{C}=0.0071 \mathrm{~A}
$$

Wavelength $=0.71073$

Cell:

$$
\begin{array}{ll}
\mathrm{a}=13.7699(16) & \mathrm{b}=17.9992(18) \\
\text { alpha }=90 & \mathrm{beta}=90
\end{array}
$$$$
\mathrm{c}=19.133(3)
$$

gamma $=90$

Temperature: $273 \mathrm{~K}$

$\begin{array}{lll} & \text { Calculated } & \text { Reported } \\ \text { Volume } & 4742.1(10) & 4742.1(10) \\ \text { Space group } & \mathrm{P} \mathrm{b} \mathrm{c} \mathrm{a} & \mathrm{P} \mathrm{b} \mathrm{c} \mathrm{a} \\ \text { Hall group } & -\mathrm{P} \mathrm{2ac} \mathrm{2ab} & -\mathrm{P} \mathrm{2ac} \mathrm{2ab} \\ \text { Moiety formula } & \mathrm{C}_{30} \mathrm{H}_{30} \mathrm{~N}_{2} \mathrm{O} & \mathrm{C}_{30} \mathrm{H}_{30} \mathrm{~N}_{2} \mathrm{O} \\ \text { Sum formula } & \mathrm{C}_{30} \mathrm{H}_{30} \mathrm{~N}_{2} \mathrm{O} & \mathrm{C}_{30} \mathrm{H}_{30} \mathrm{~N}_{2} \mathrm{O} \\ \mathrm{Mr} & 434.56 & 434.56 \\ \text { Dx,g cm-3 } & 1.217 & 1.217 \\ Z & 8 & 8\end{array}$




\begin{tabular}{|c|c|c|}
\hline $\mathrm{Mu}(\mathrm{mm}-1)$ & 0.073 & 0.073 \\
\hline F000 & 1856.0 & 1856.0 \\
\hline F000' & 1856.68 & \\
\hline $\mathrm{h}, \mathrm{k}, \operatorname{lmax}$ & $16,21,22$ & $16,21,22$ \\
\hline Nref & 4238 & 4237 \\
\hline Tmin,Tmax & $0.989,0.993$ & $0.531,0.746$ \\
\hline Tmin' & 0.986 & \\
\hline \multicolumn{3}{|c|}{$\begin{array}{l}\text { Correction method }=\text { \# Reported T Limits: } \text { Tmin }=0.531 \text { Tmax }=0.746 \text { AbsCorr }= \\
\text { MULTI-SCAN }\end{array}$} \\
\hline \multicolumn{2}{|c|}{ Data completeness $=1.000$} & \\
\hline \multicolumn{2}{|c|}{$\mathrm{R}($ reflections $)=0.0768(1715)$} & 2023( 4237) \\
\hline$S=1.016$ & Npar $=31$ & \\
\hline
\end{tabular}

\subsection{Crystal structure of 9a}

Preparation of the single crystals of 9a: $15.0 \mathrm{mg}$ of pure compound 9a was dissolved in the combined solvents of $\mathrm{CH}_{2} \mathrm{Cl}_{2}$ and $\mathrm{EtOH}(6 \mathrm{~mL}, \mathrm{v} / \mathrm{v}=1: 1)$ at room temperature. The bottle was sealed by a piece of plastic film with three tiny holes, thus allowing slow evaporation of the solvents at room temperature. After about one week, several small particles were observed at the bottom of the bottle. The crystals were chosen and subjected to the single crystal X-ray diffraction analysis for the determination of the structure and relative configuration of 9a. The data were collected by a Rigaku Gemini E at 293.0 K.

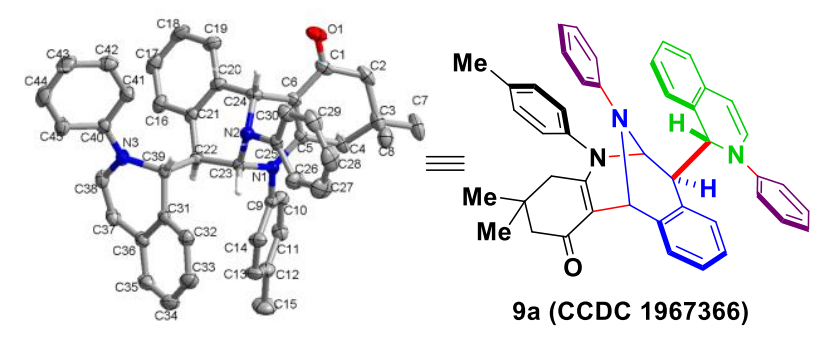

Displacement ellipsoids are drawn at the $30 \%$ probability level.

\begin{tabular}{llll} 
Bond precision: & $\mathrm{C}-\mathrm{C}=0.0030 \mathrm{~A}$ & \multicolumn{1}{c}{ Wavelength=1.54184 } \\
Cell: & $\mathrm{a}=9.5513(2)$ & $\mathrm{b}=22.5642(6)$ & $\mathrm{c}=16.2921(5)$ \\
& alpha $=90$ & beta=101.061(3) & gamma $=90$
\end{tabular}

Temperature: $293 \mathrm{~K}$

\section{Calculated}

Volume

Space group

Hall group
3446.01(16)

P 21/c

-P 2ybc
Reported

3446.01(16)

P 1 21/c 1

-P 2ybc 


\begin{tabular}{|c|c|c|}
\hline Moiety formula & $\mathrm{C}_{45} \mathrm{H}_{41} \mathrm{~N}_{3} \mathrm{O}$ & $\mathrm{C}_{45} \mathrm{H}_{41} \mathrm{~N}_{3} \mathrm{O}$ \\
\hline Sum formula & $\mathrm{C}_{45} \mathrm{H}_{41} \mathrm{~N}_{3} \mathrm{O}$ & $\mathrm{C}_{45} \mathrm{H}_{41} \mathrm{~N}_{3} \mathrm{O}$ \\
\hline $\mathrm{Mr}$ & 639.81 & 639.81 \\
\hline Dx,g cm-3 & 1.233 & 1.233 \\
\hline $\mathrm{Z}$ & 4 & 4 \\
\hline $\mathrm{Mu}(\mathrm{mm}-1)$ & 0.568 & 0.568 \\
\hline F000 & 1360.0 & 1360.0 \\
\hline F000' & 1363.61 & \\
\hline $\mathrm{h}, \mathrm{k}, \operatorname{lmax}$ & $11,26,19$ & $11,26,19$ \\
\hline Nref & 6168 & 6167 \\
\hline Tmin,Tmax & $0.903,0.939$ & $0.915,1.000$ \\
\hline Tmin' & 0.898 & \\
\hline \multicolumn{3}{|c|}{$\begin{array}{l}\text { Correction method }=\text { \# Reported T Limits: Tmin }=0.915 \text { Tmax }=1.000 \text { AbsCorr }= \\
\text { MULTI-SCAN }\end{array}$} \\
\hline \multicolumn{2}{|c|}{ Data completeness $=1.000$} & \\
\hline \multicolumn{2}{|c|}{$\mathrm{R}($ reflections $)=0.0486(4904)$} & 1448( 6167) \\
\hline$S=1.029$ & Npar $=4$ & \\
\hline
\end{tabular}




\section{9. ${ }^{1} \mathrm{H}$ NMR and ${ }^{13} \mathrm{C}$ NMR spectra}

${ }^{1} \mathrm{H}$ NMR spectrum of $\mathbf{4 a}\left(400 \mathrm{MHz}, \mathrm{CDCl}_{3}\right)$

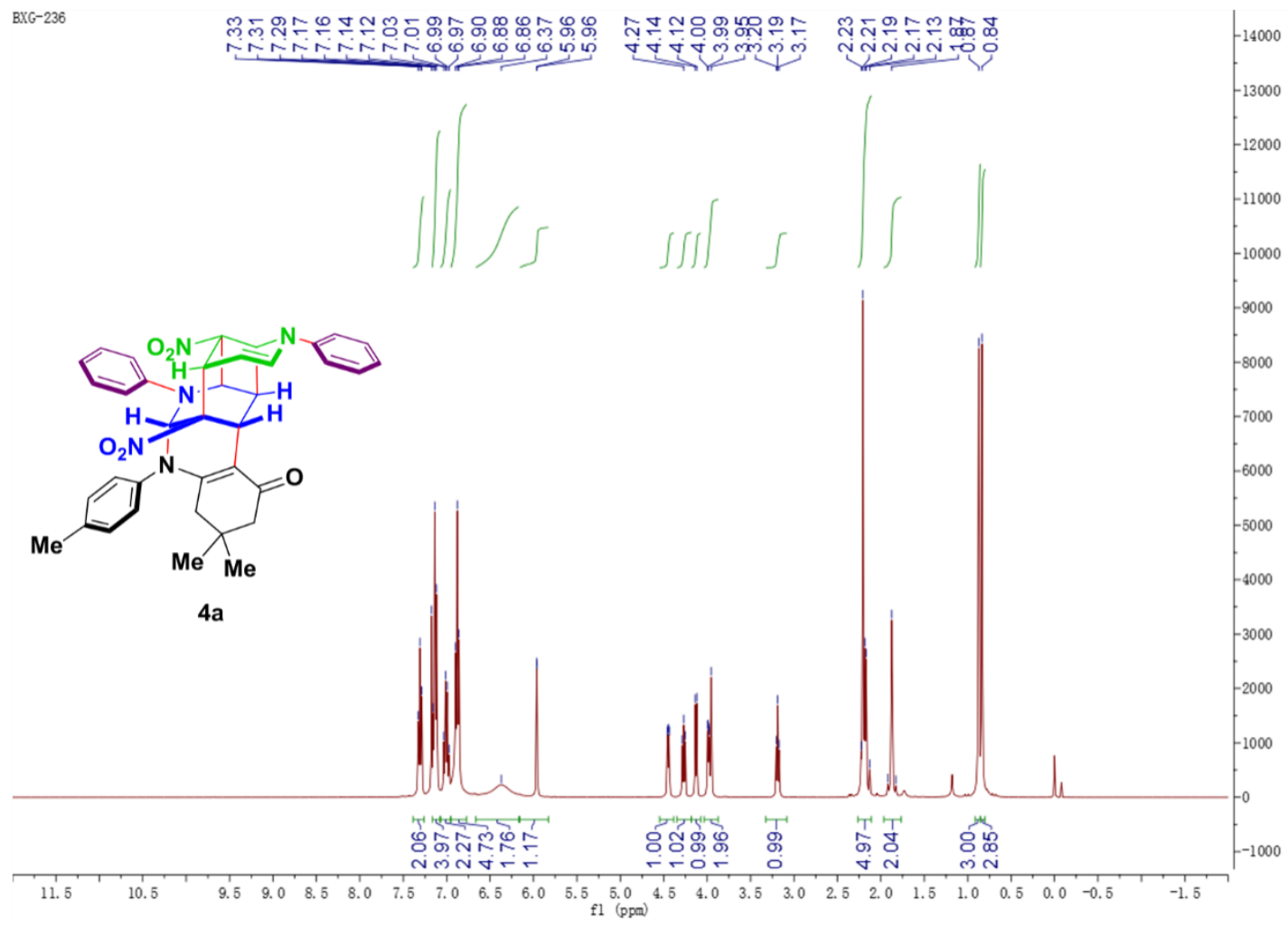

${ }^{13} \mathrm{C}$ NMR spectrum of $\mathbf{4 a}\left(100 \mathrm{MHz}, \mathrm{CDCl}_{3}\right)$ 


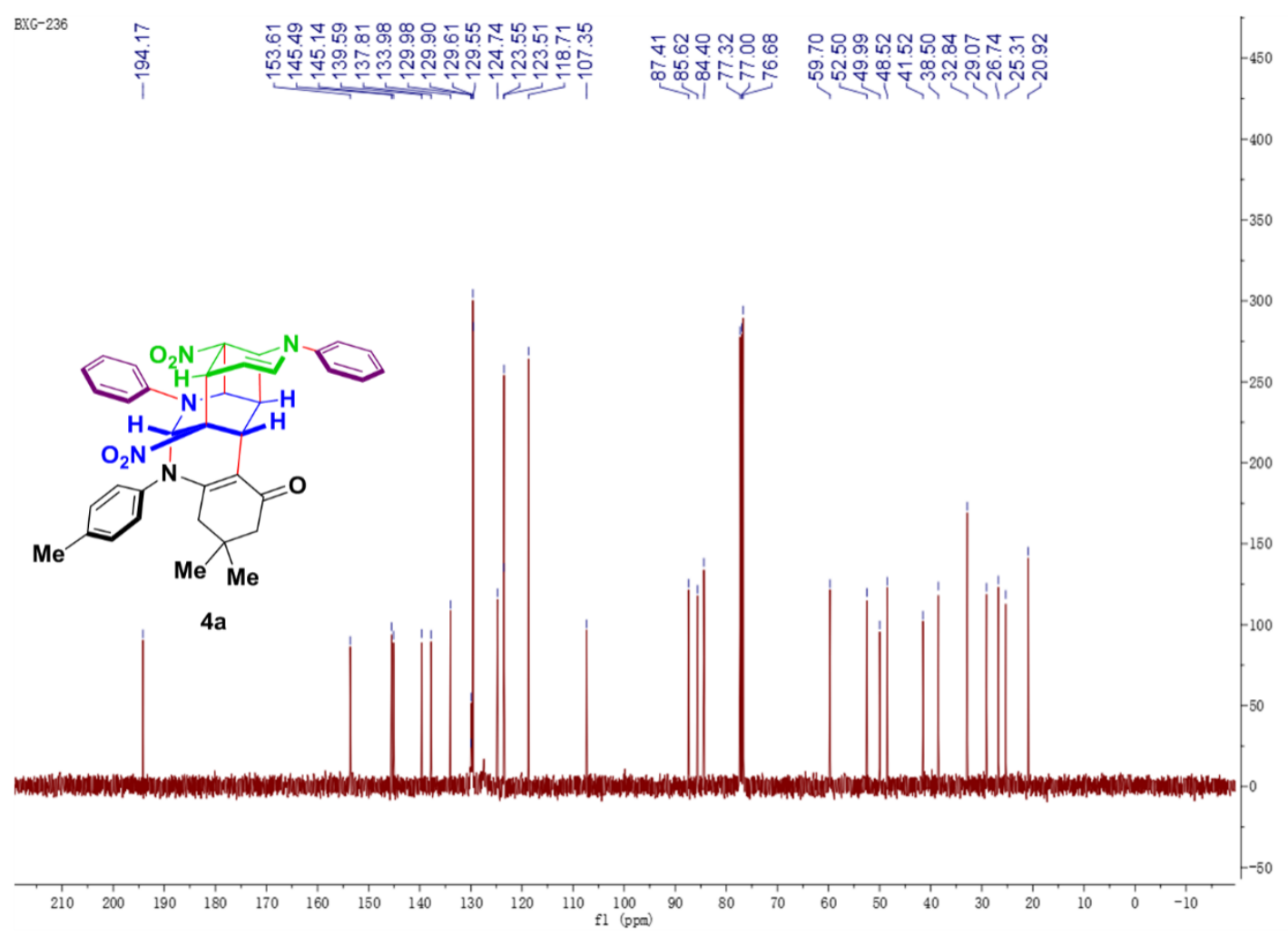

${ }^{1} \mathrm{H}$ NMR spectrum of $\mathbf{4 b}\left(400 \mathrm{MHz}, \mathrm{CDCl}_{3}\right)$

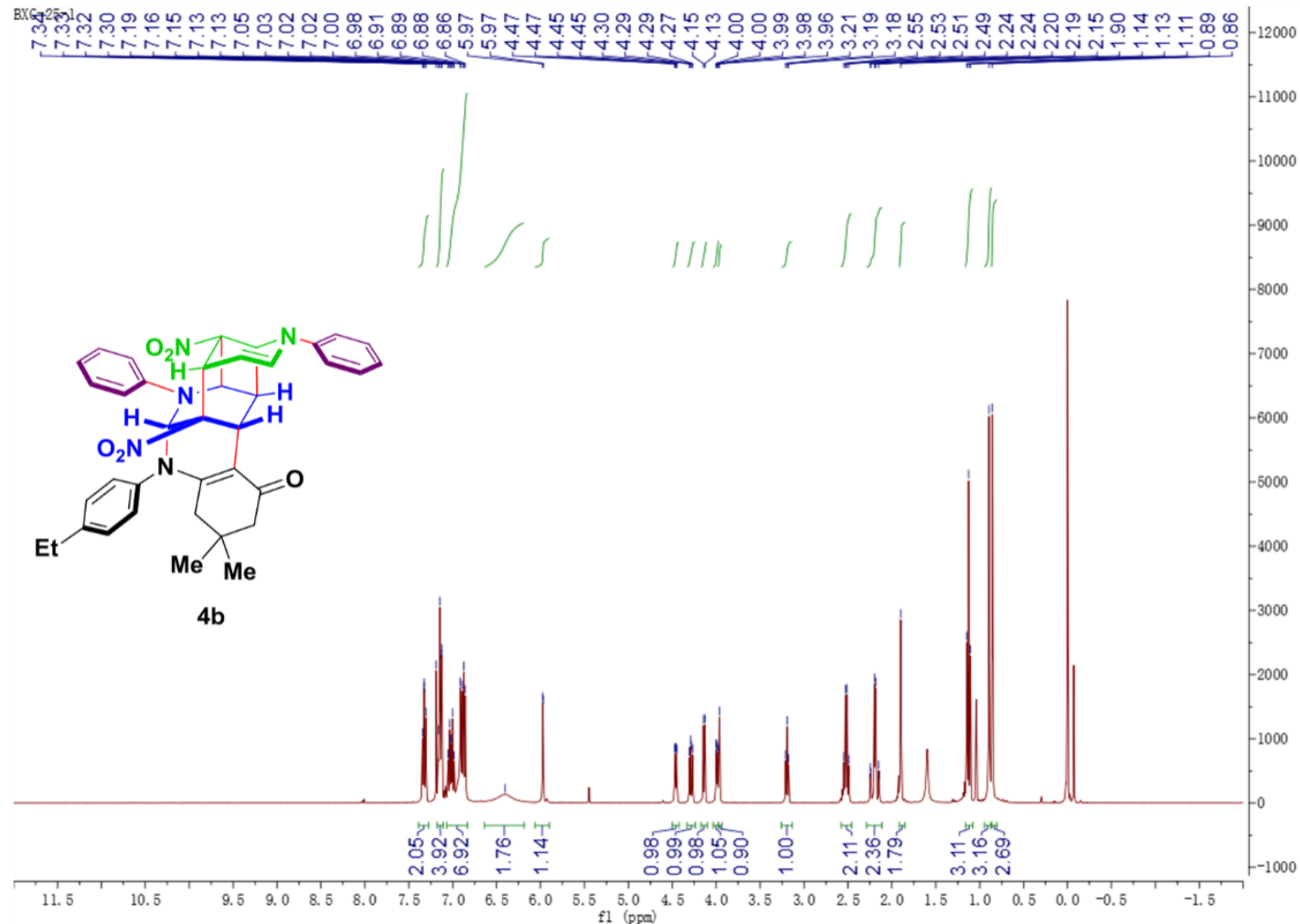

${ }^{13} \mathrm{C}$ NMR spectrum of $\mathbf{4 b}\left(100 \mathrm{MHz}, \mathrm{CDCl}_{3}\right)$ 


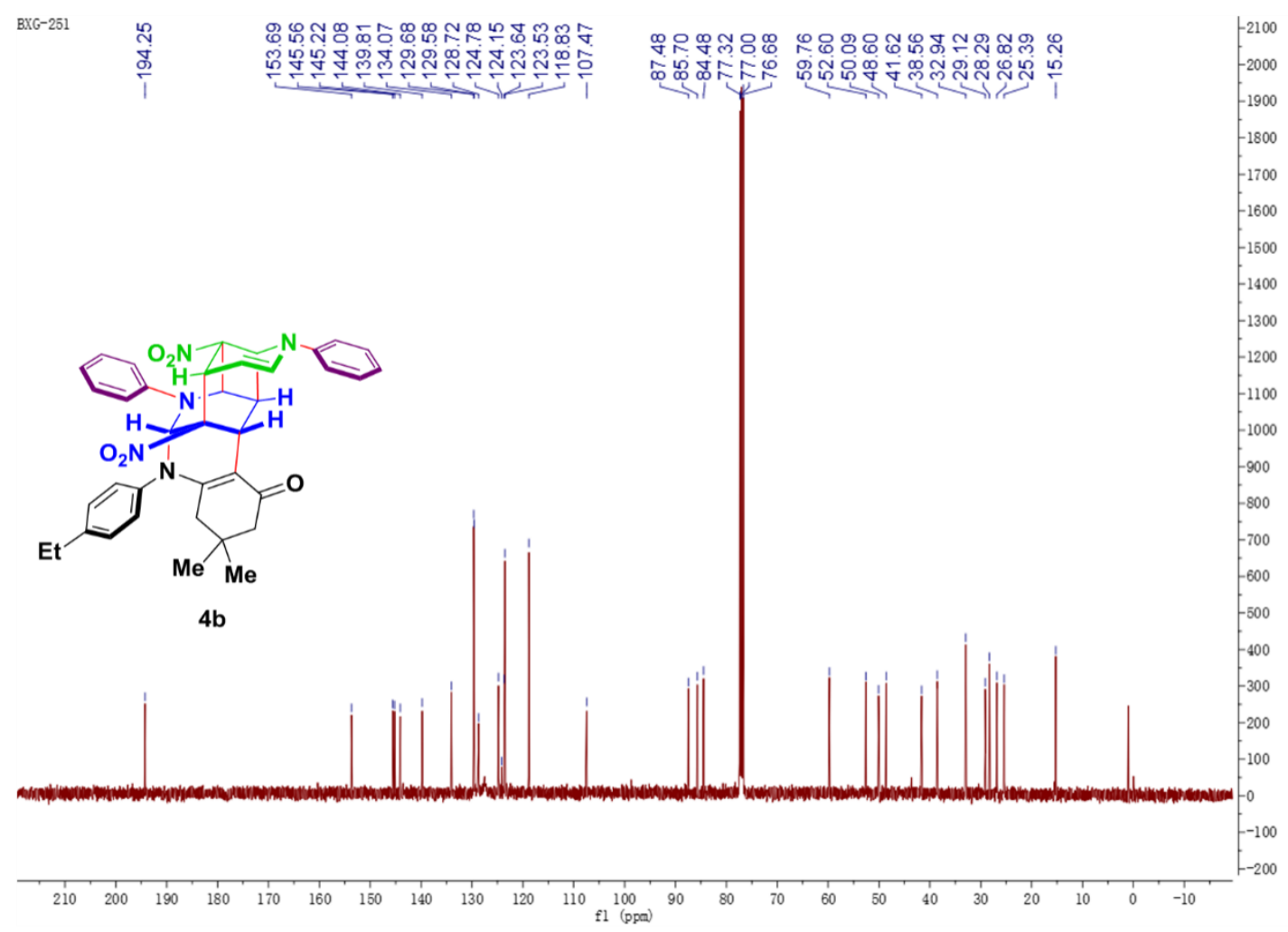

${ }^{1} \mathrm{H}$ NMR spectrum of $\mathbf{4 c}\left(400 \mathrm{MHz}, \mathrm{CDCl}_{3}\right)$

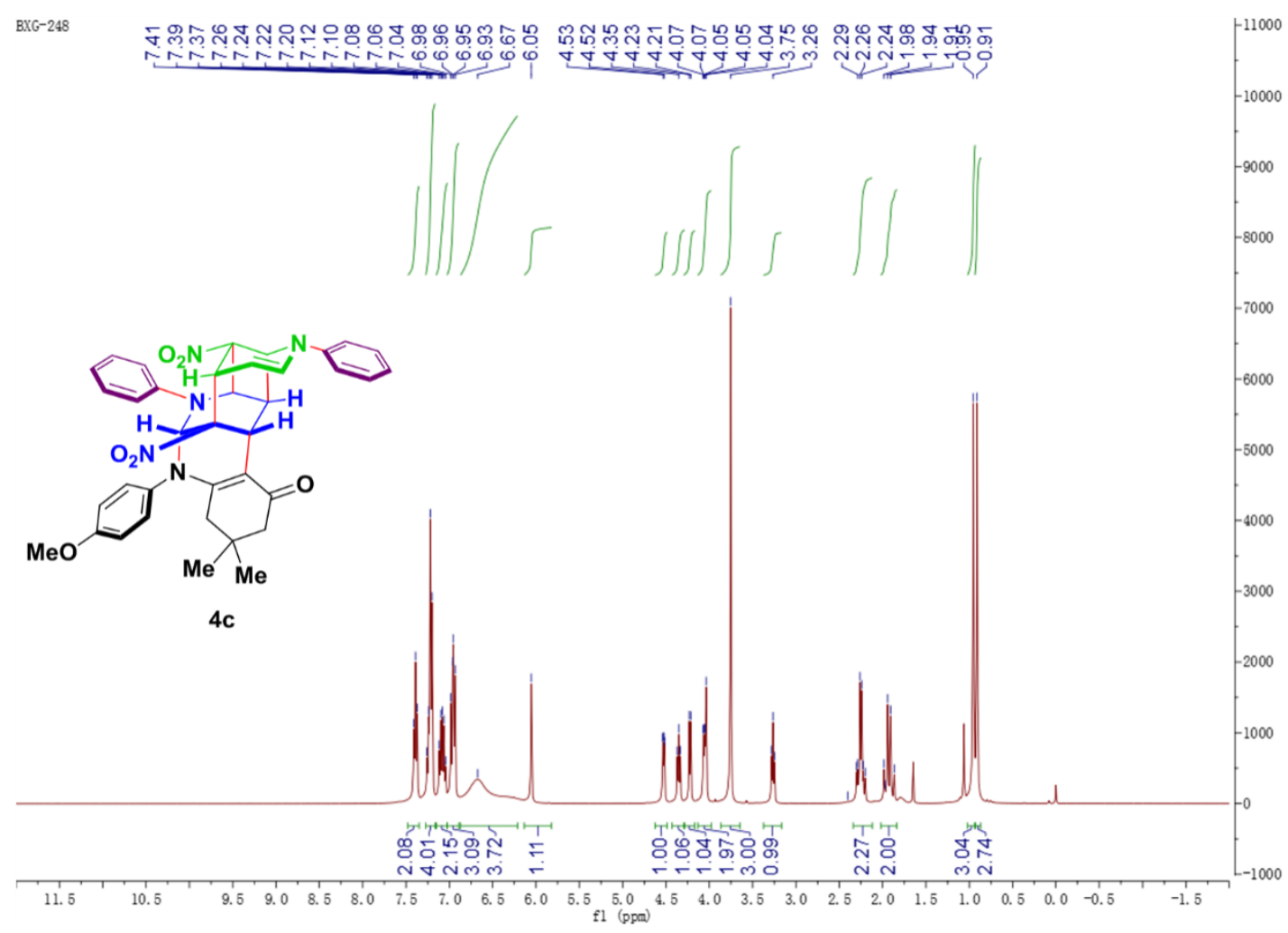

${ }^{13} \mathrm{C}$ NMR spectrum of $\mathbf{4 c}\left(100 \mathrm{MHz}, \mathrm{CDCl}_{3}\right)$ 


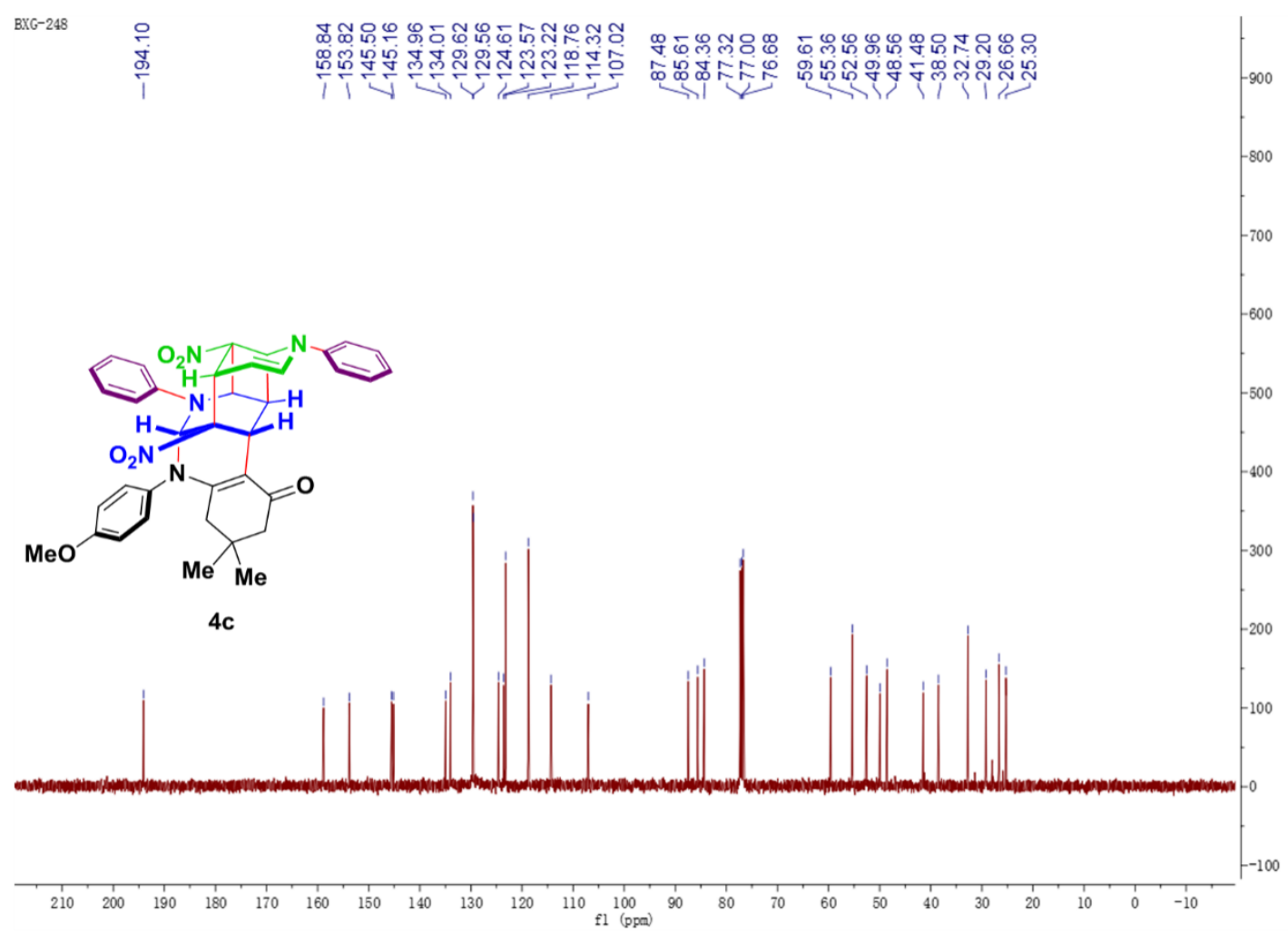

${ }^{1} \mathrm{H}$ NMR spectrum of $\mathbf{4 d}\left(400 \mathrm{MHz}, \mathrm{CDCl}_{3}\right)$

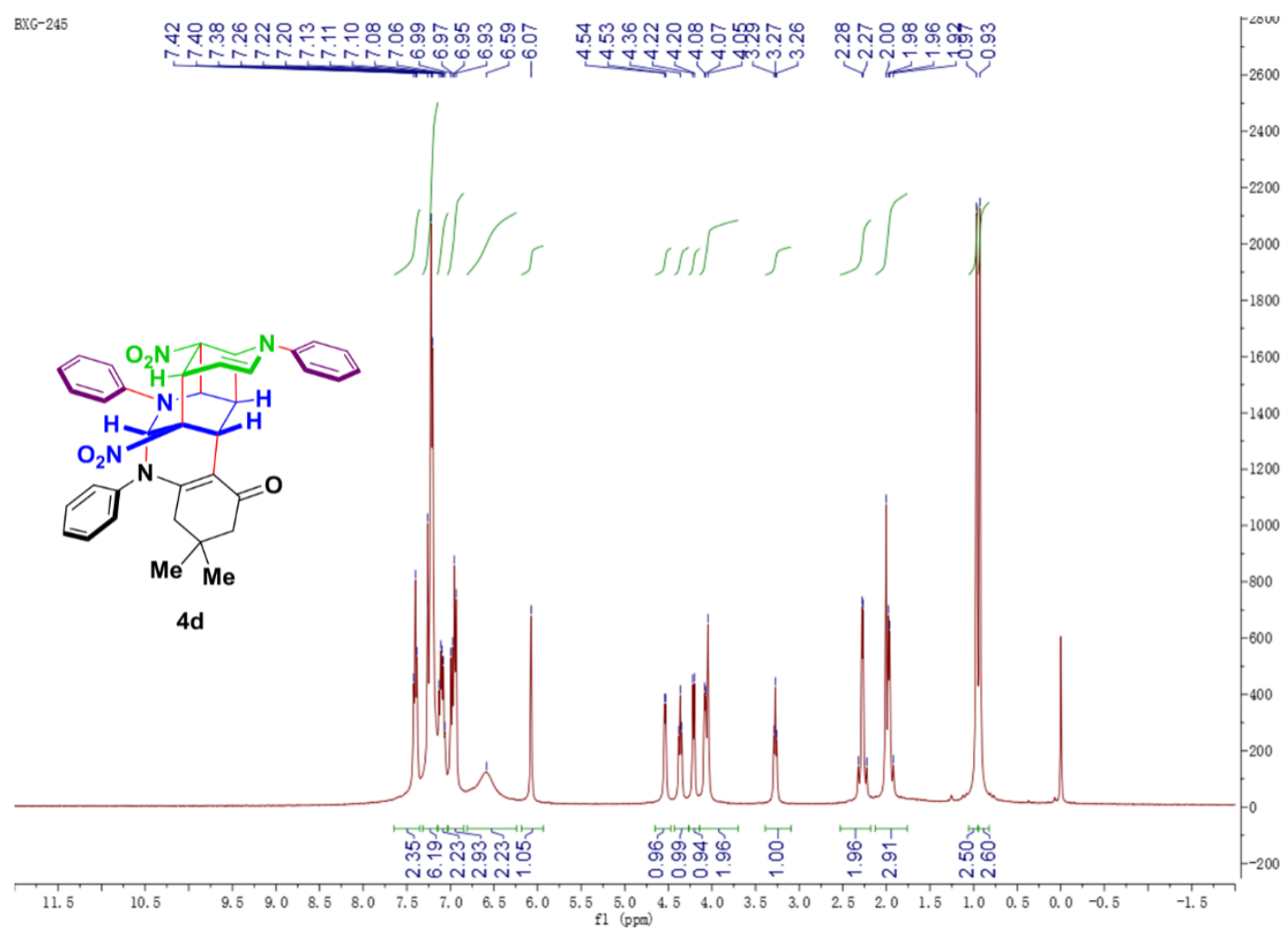

${ }^{13} \mathrm{C}$ NMR spectrum of $\mathbf{4 d}\left(100 \mathrm{MHz}, \mathrm{CDCl}_{3}\right)$ 


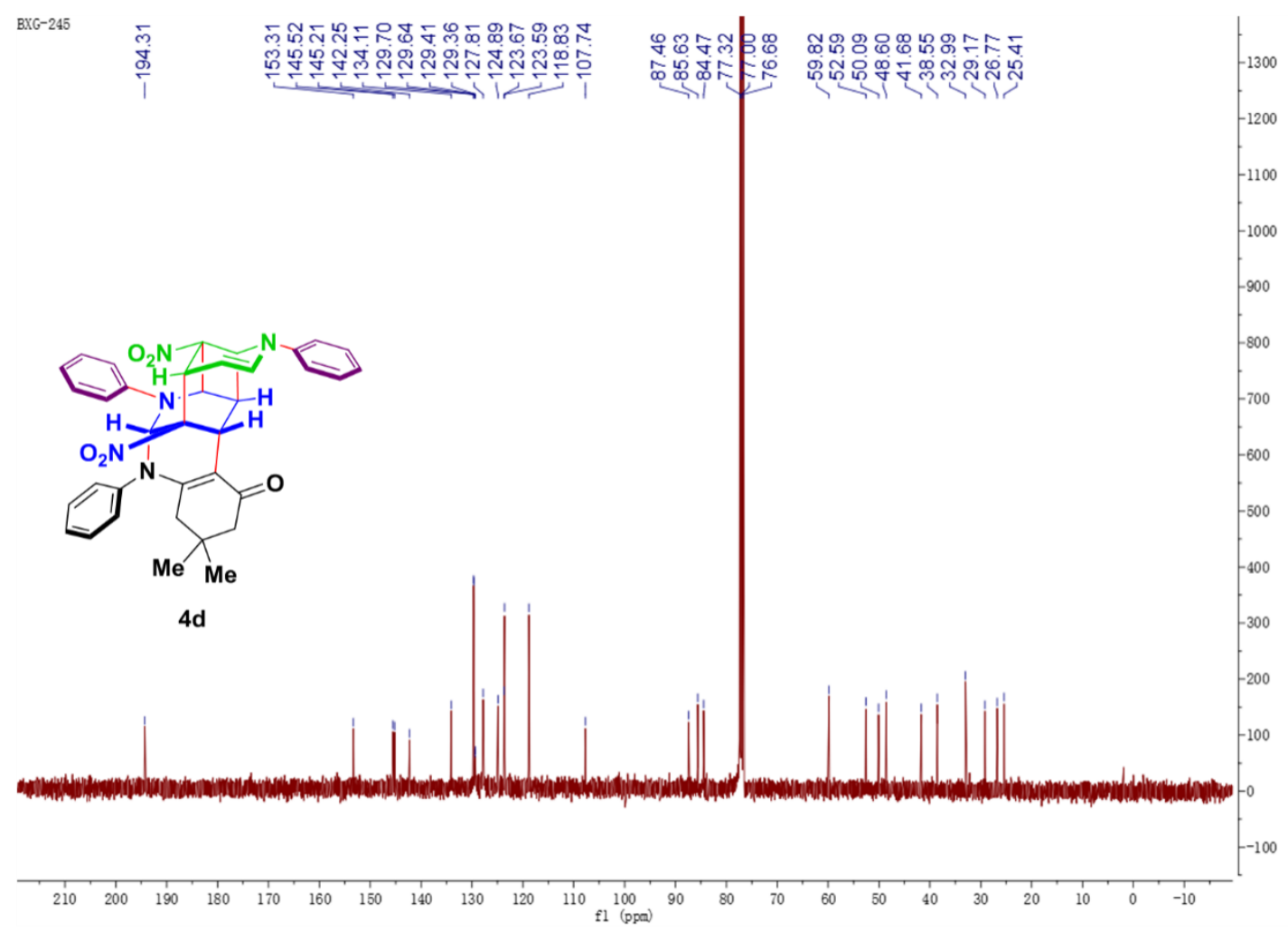

${ }^{1} \mathrm{H}$ NMR spectrum of $4 \mathbf{e}\left(400 \mathrm{MHz}, \mathrm{CDCl}_{3}\right)$

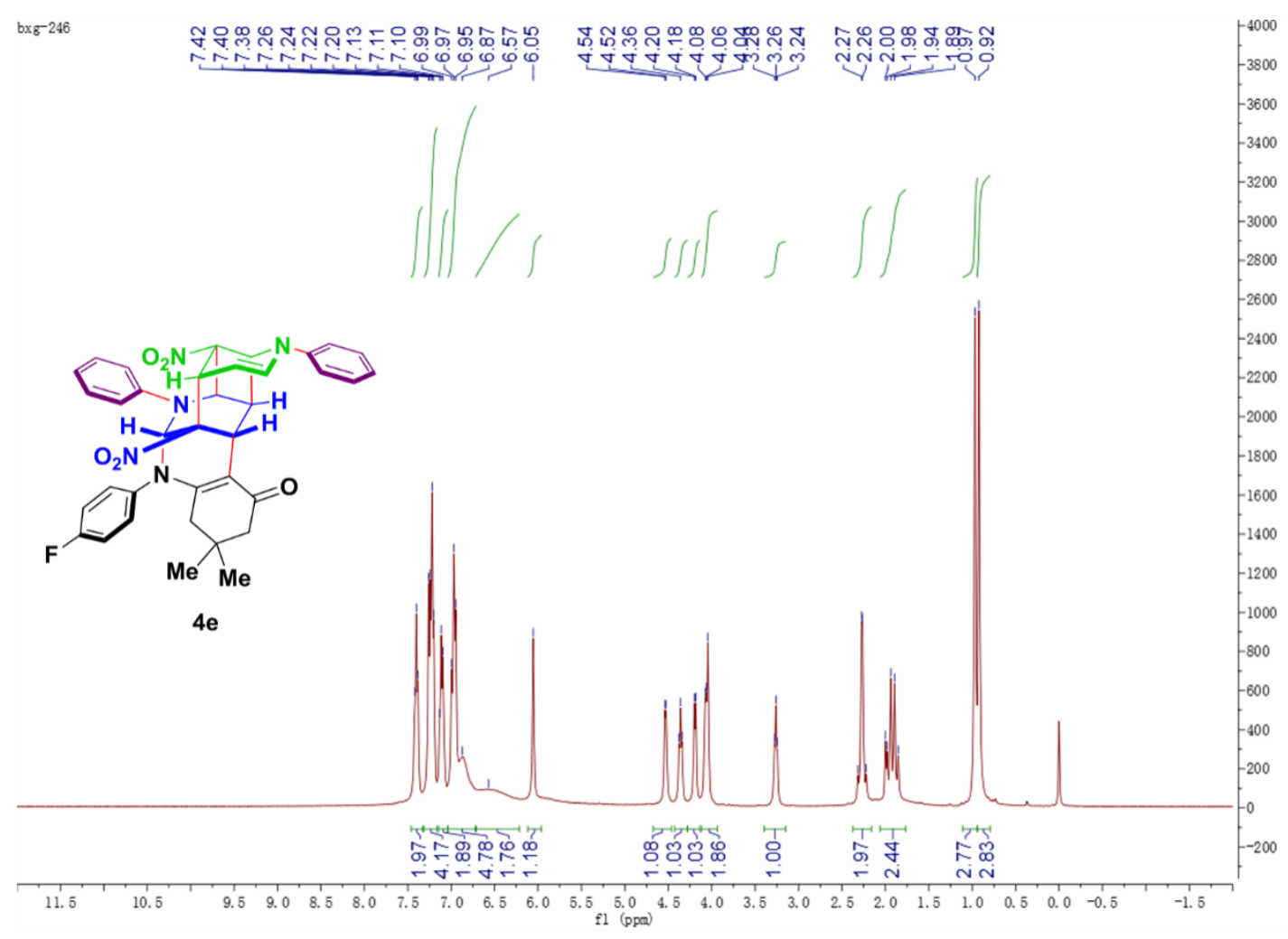

${ }^{13} \mathrm{C}$ NMR spectrum of $4 \mathbf{e}\left(100 \mathrm{MHz}, \mathrm{CDCl}_{3}\right)$ 


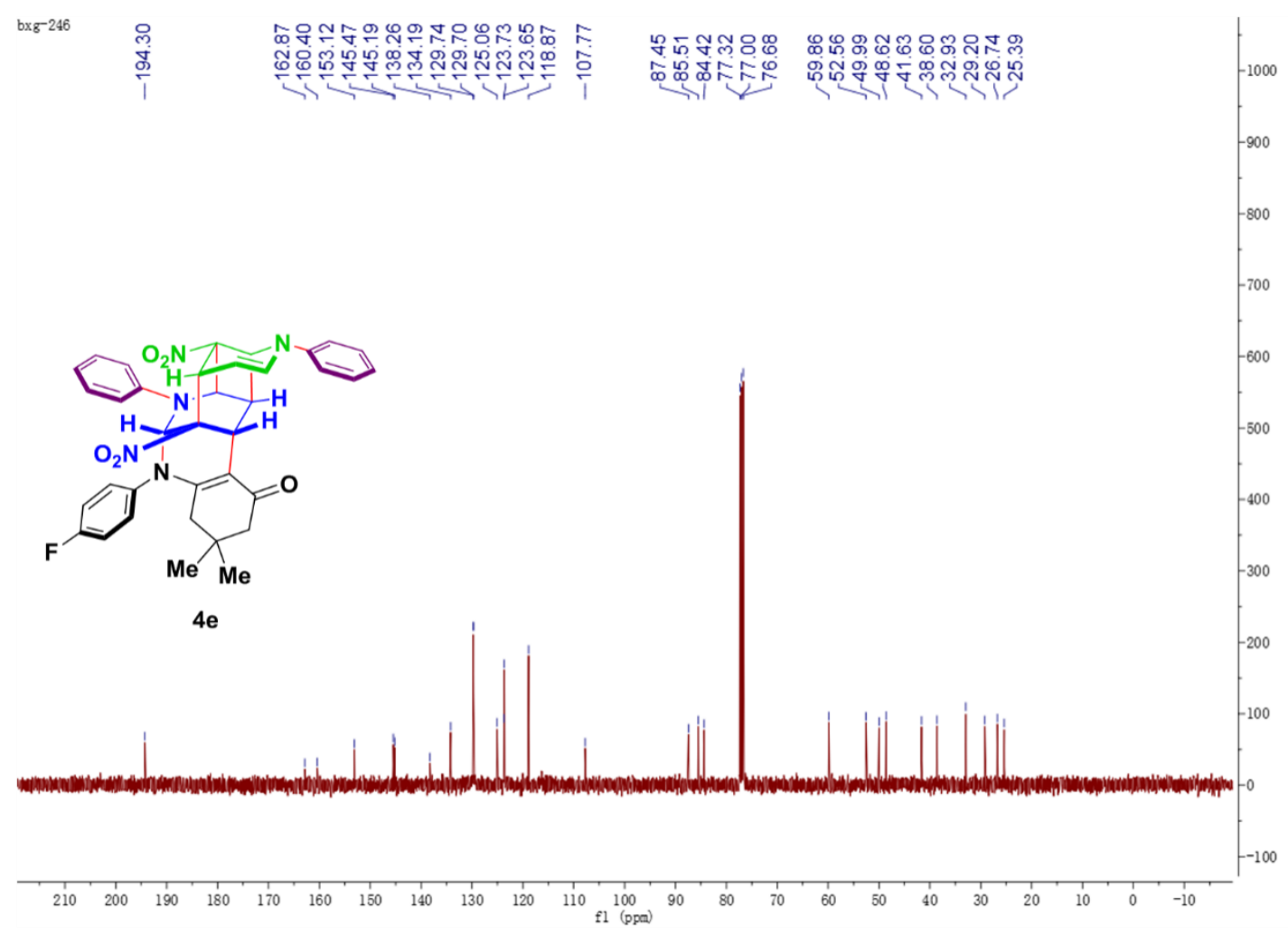

${ }^{1} \mathrm{H}$ NMR spectrum of $\mathbf{4 f}\left(400 \mathrm{MHz}, \mathrm{CDCl}_{3}\right)$

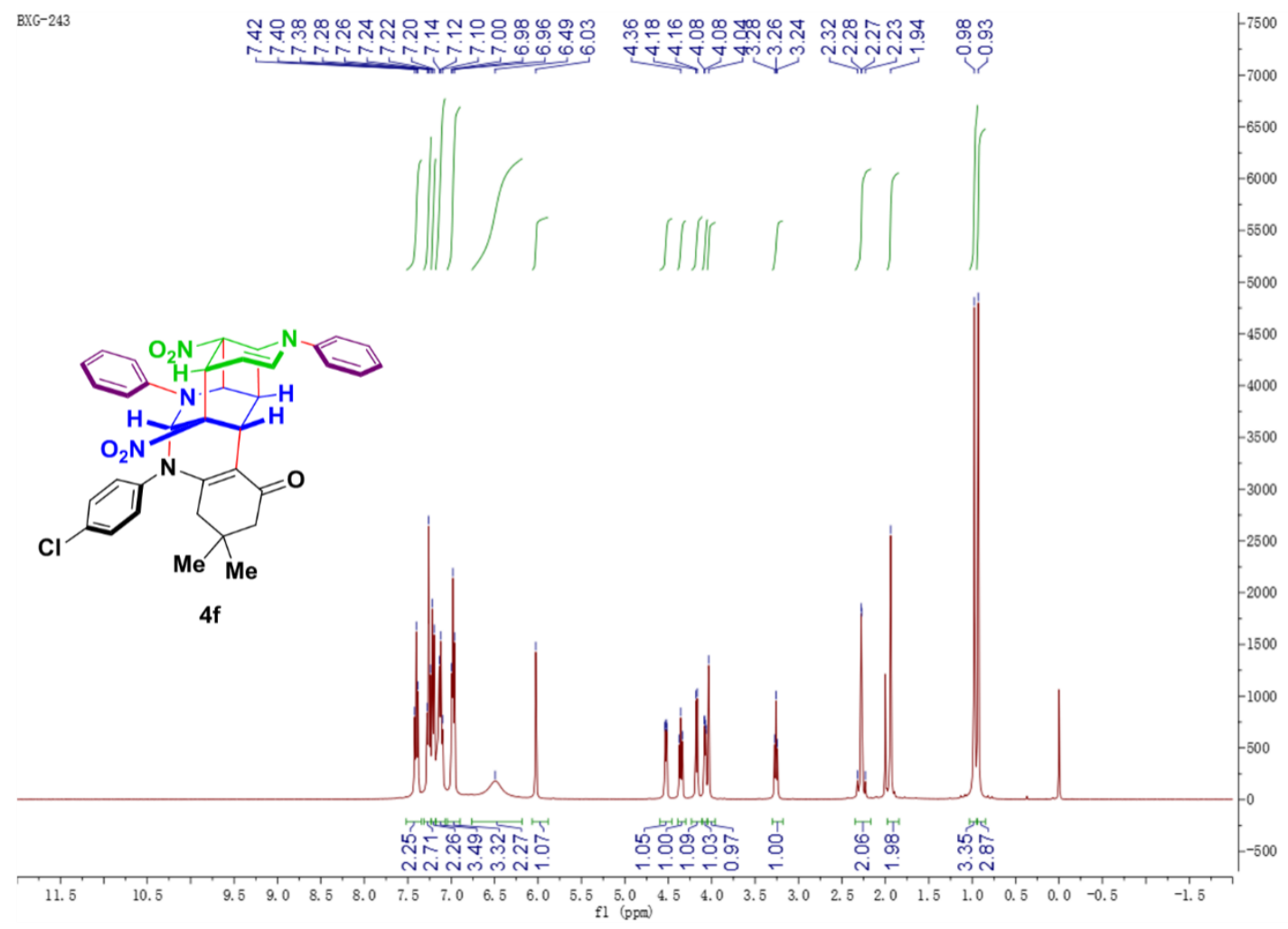

${ }^{13} \mathrm{C}$ NMR spectrum of $\mathbf{4 f}\left(100 \mathrm{MHz}, \mathrm{CDCl}_{3}\right)$ 


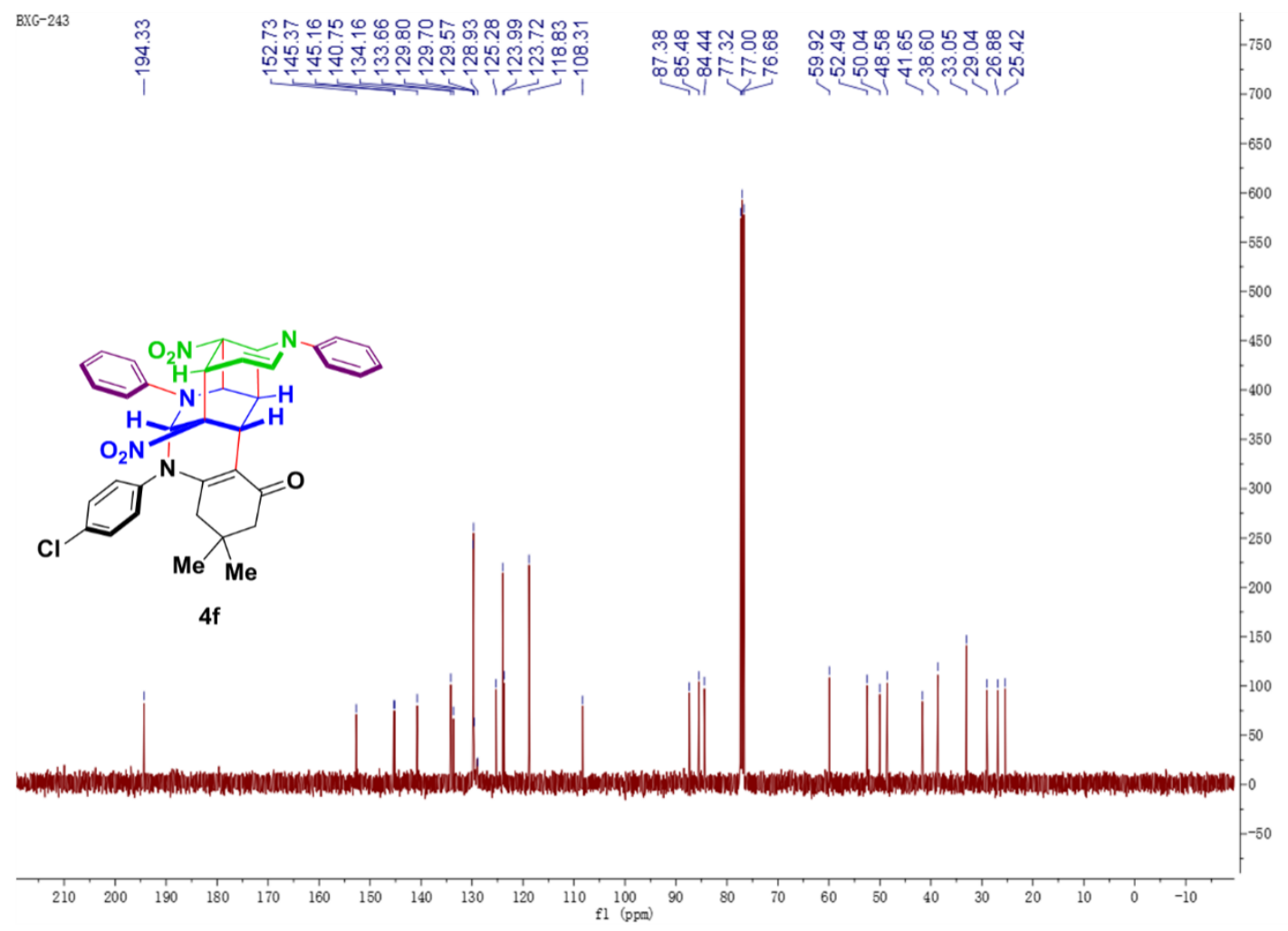

${ }^{1} \mathrm{H}$ NMR spectrum of $\mathbf{4 g}\left(400 \mathrm{MHz}, \mathrm{CDCl}_{3}\right)$

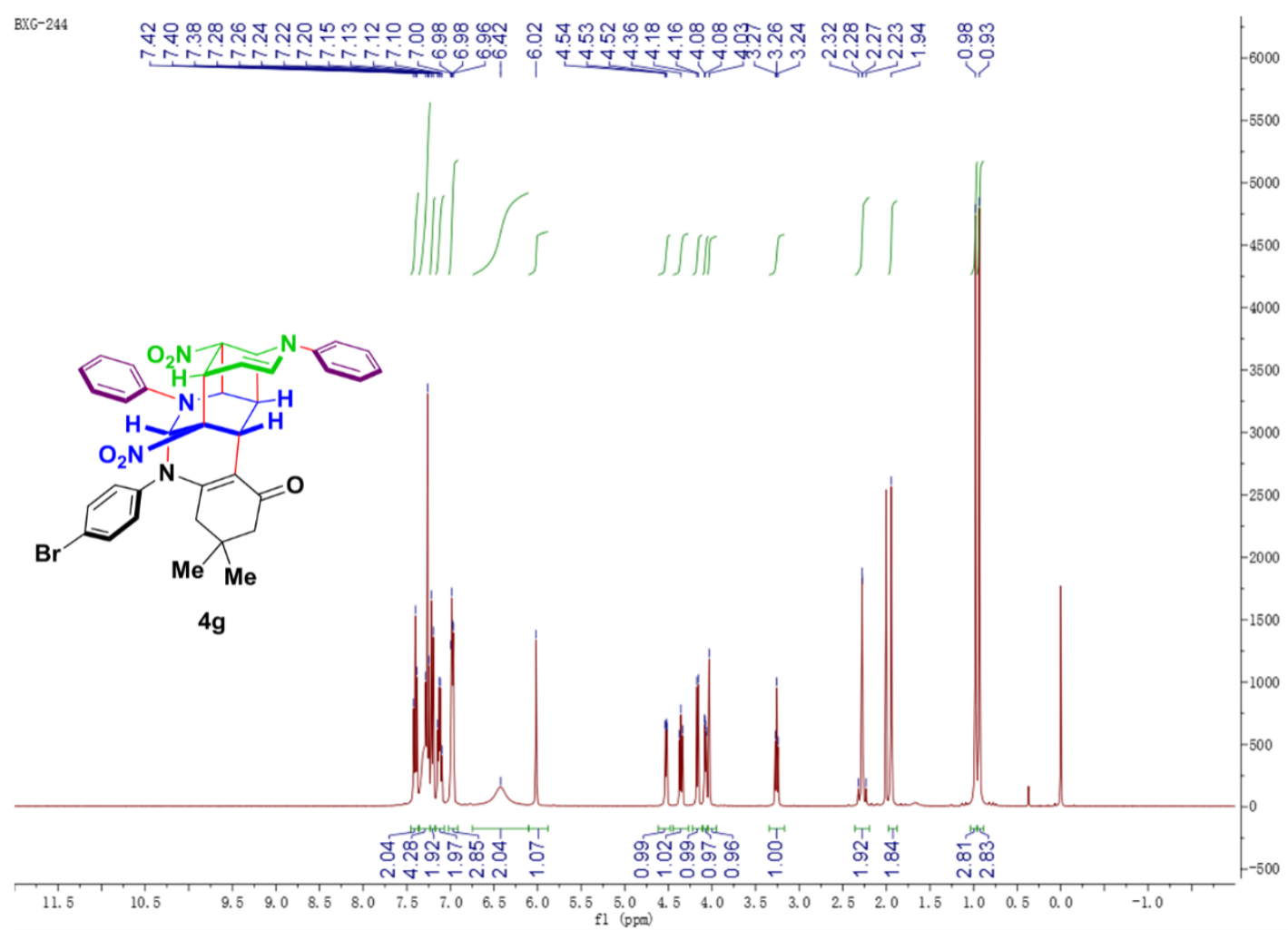

${ }^{13} \mathrm{C}$ NMR spectrum of $\mathbf{4 g}\left(100 \mathrm{MHz}, \mathrm{CDCl}_{3}\right)$ 


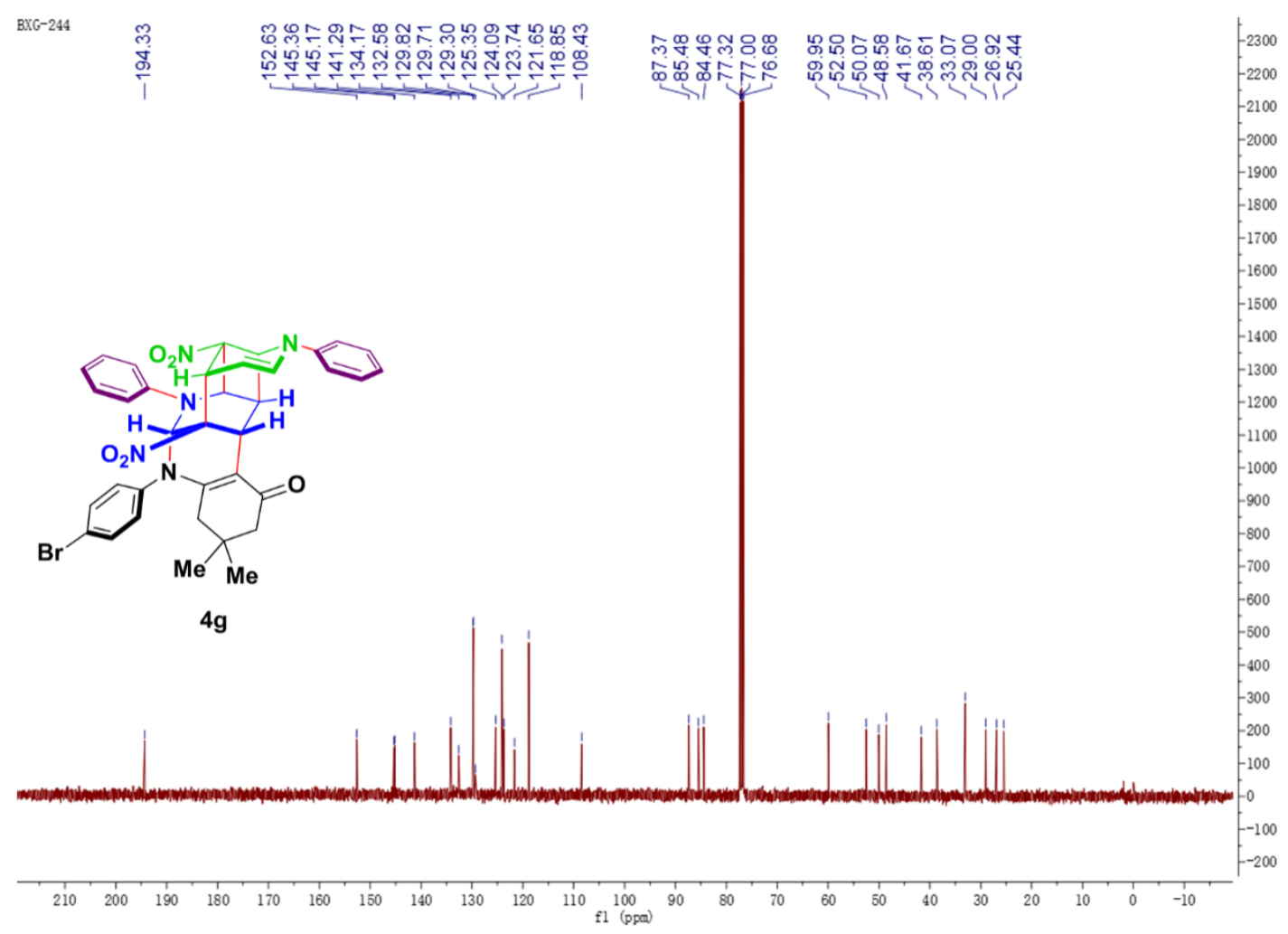

${ }^{1} \mathrm{H}$ NMR spectrum of $\mathbf{4 h}\left(400 \mathrm{MHz}, \mathrm{CDCl}_{3}\right)$

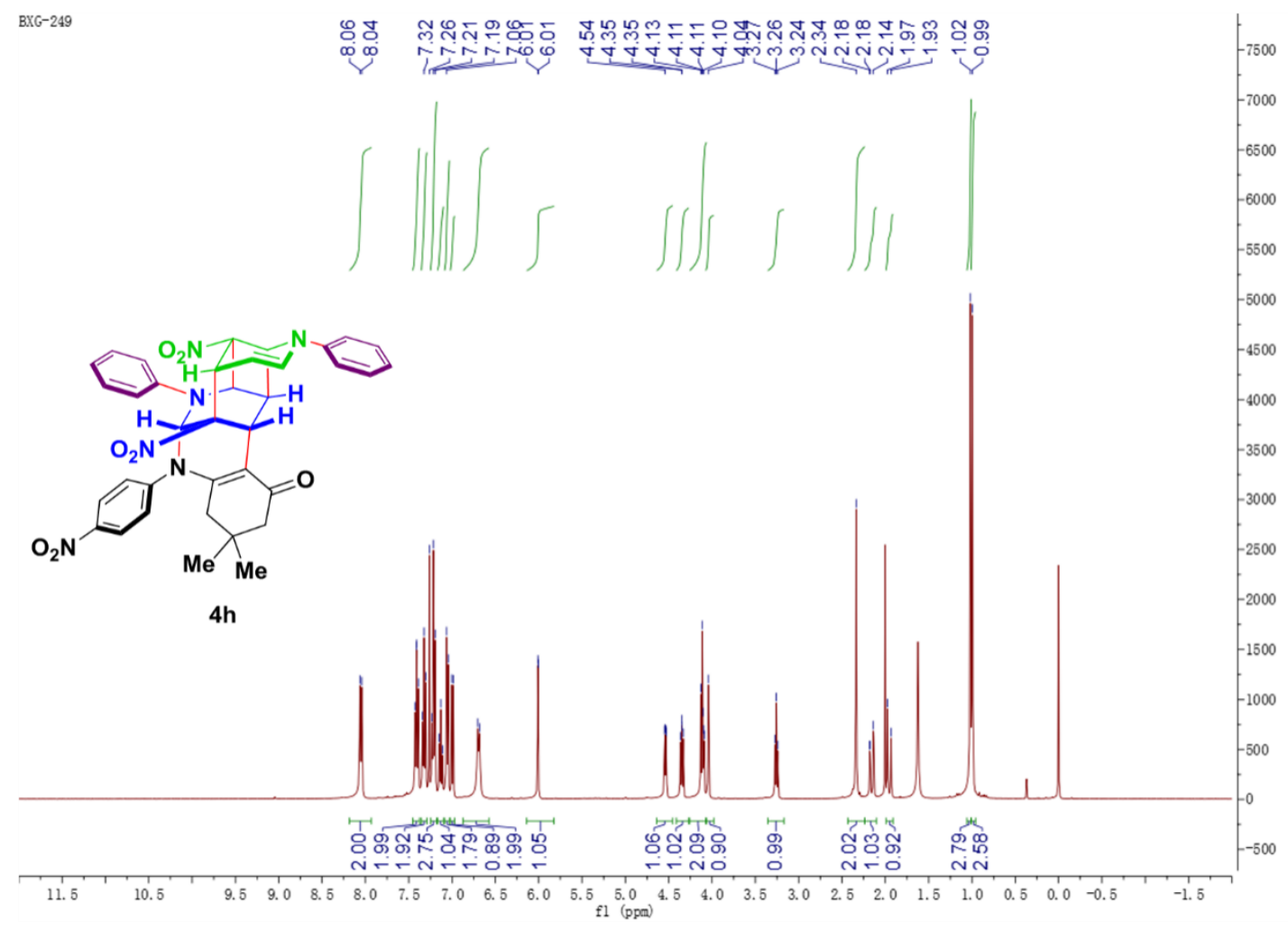

${ }^{13} \mathrm{C}$ NMR spectrum of $\mathbf{4 h}\left(100 \mathrm{MHz}, \mathrm{CDCl}_{3}\right)$ 


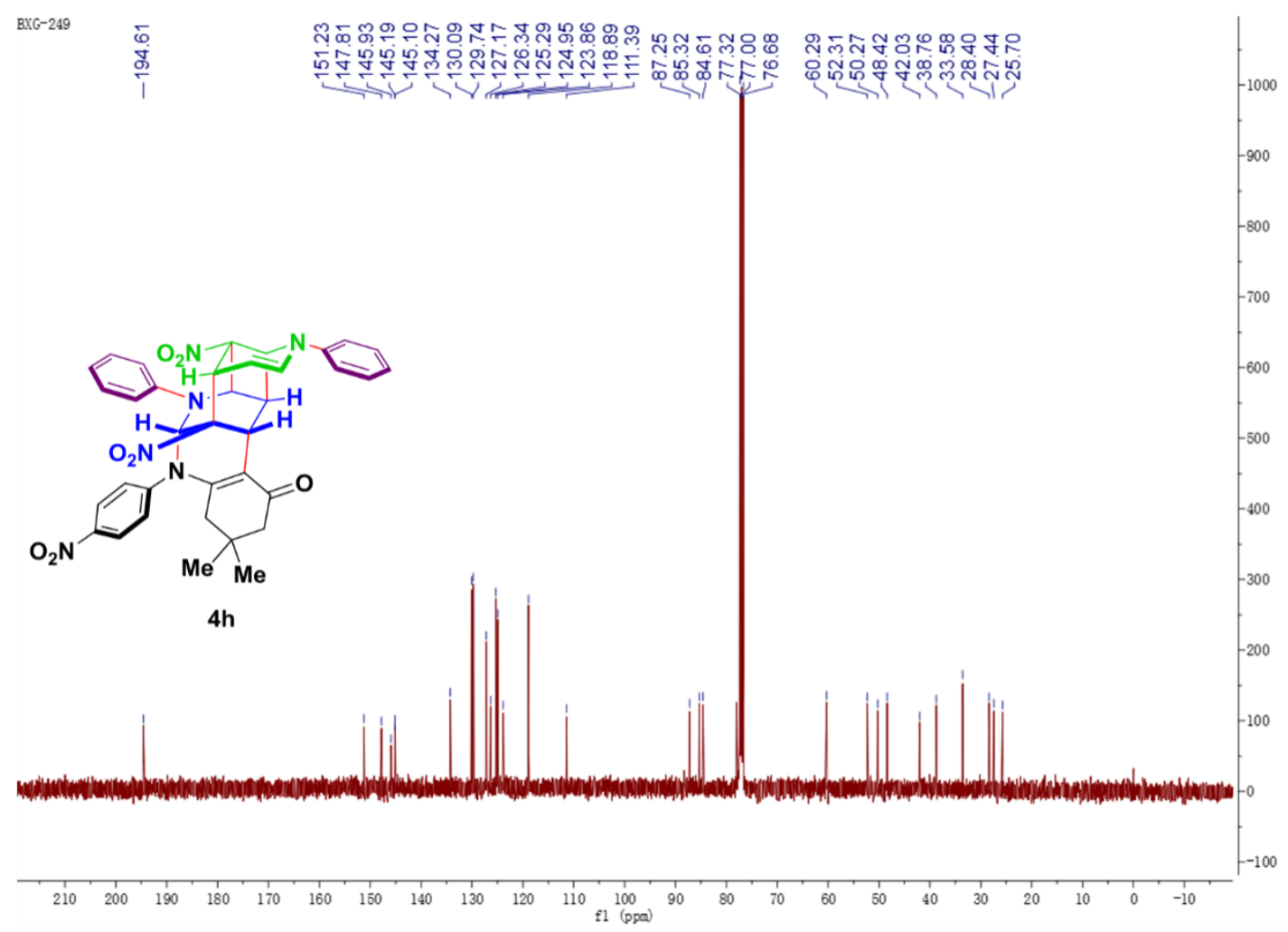

${ }^{1} \mathrm{H}$ NMR spectrum of $\mathbf{4 i}\left(400 \mathrm{MHz}, \mathrm{CDCl}_{3}\right)$

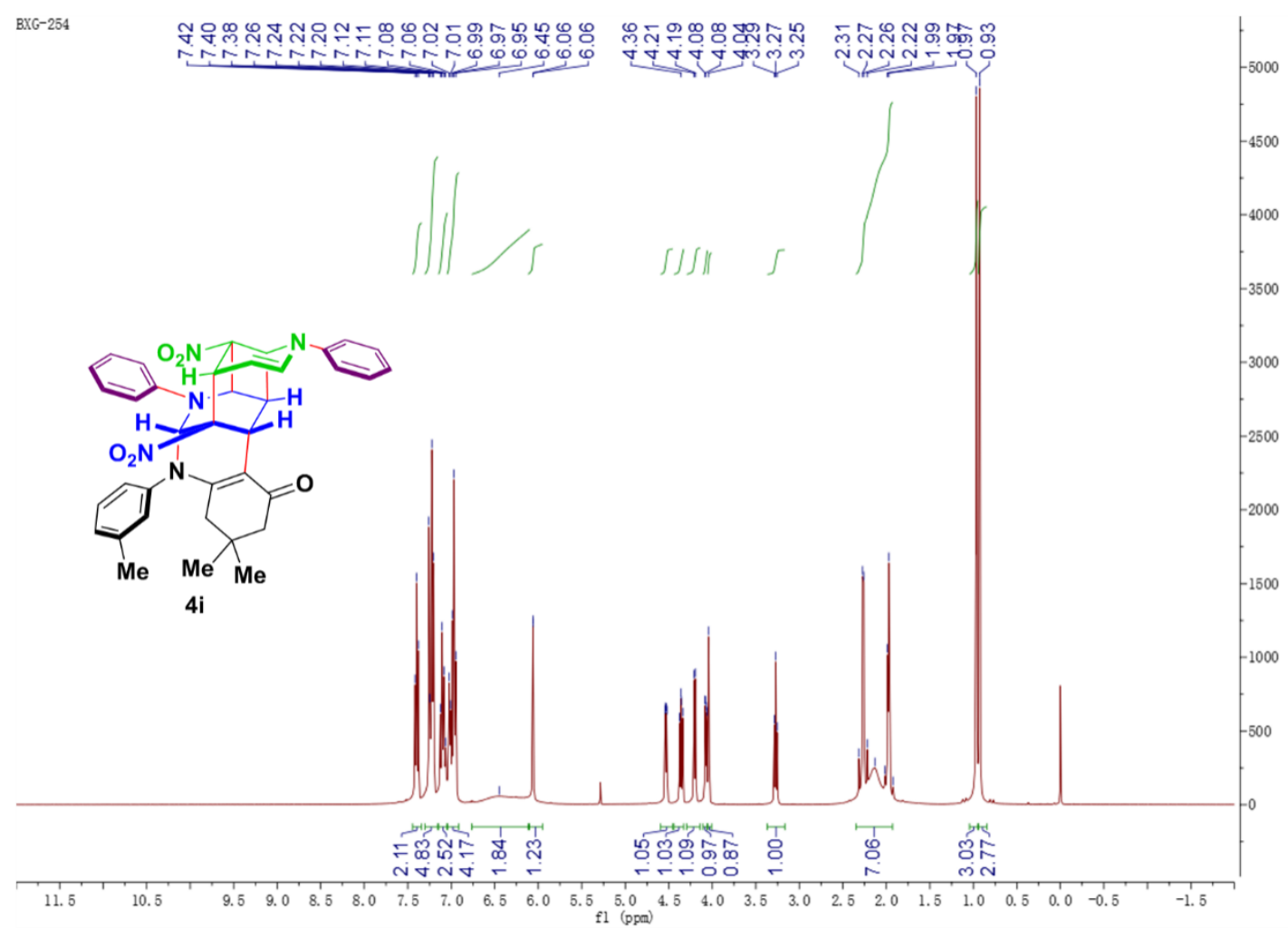

${ }^{13} \mathrm{C}$ NMR spectrum of $4 \mathbf{i}\left(100 \mathrm{MHz}, \mathrm{CDCl}_{3}\right)$ 


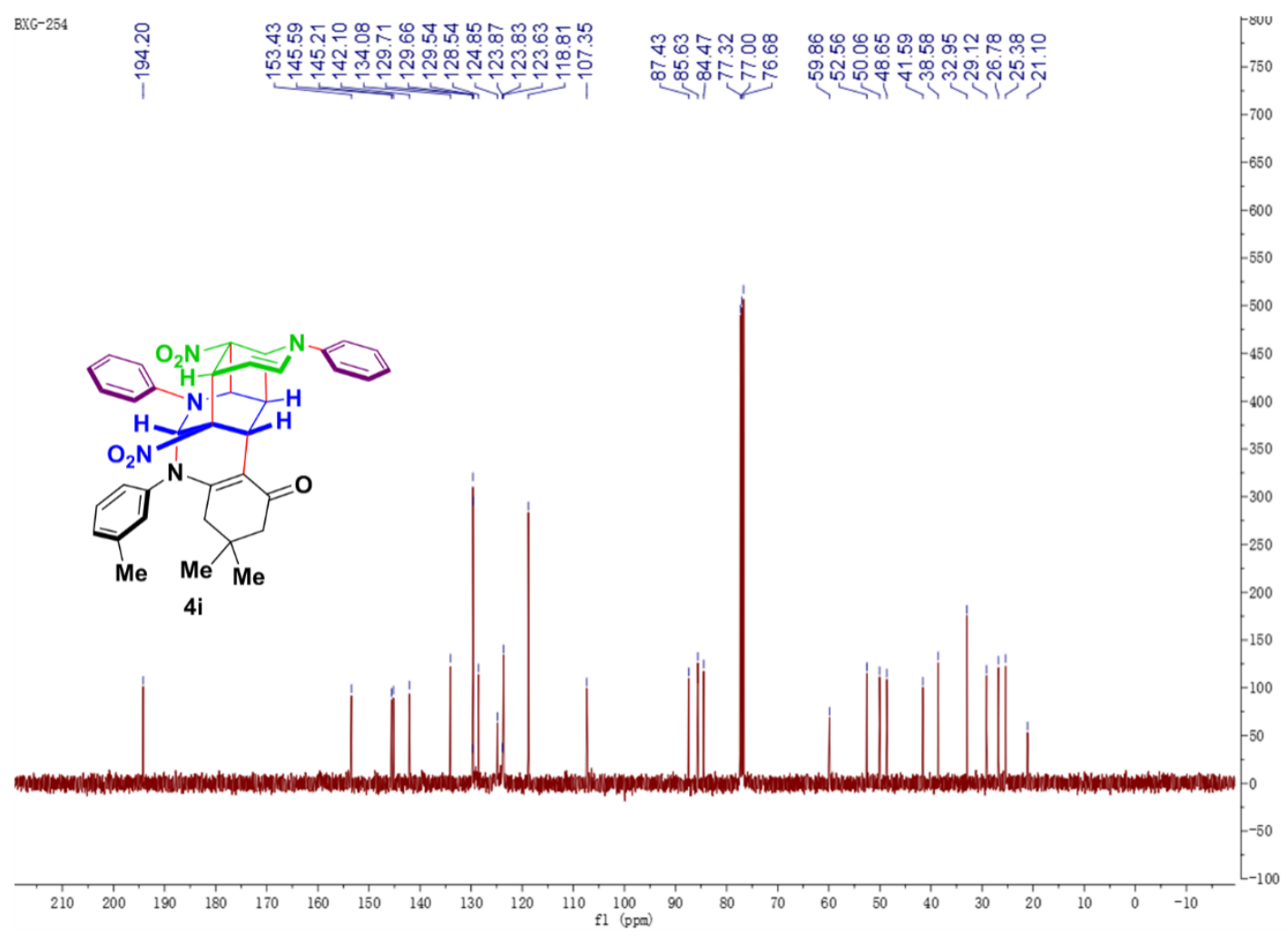

${ }^{1} \mathrm{H}$ NMR spectrum of $\mathbf{4 j}\left(400 \mathrm{MHz}, \mathrm{CDCl}_{3}\right)$

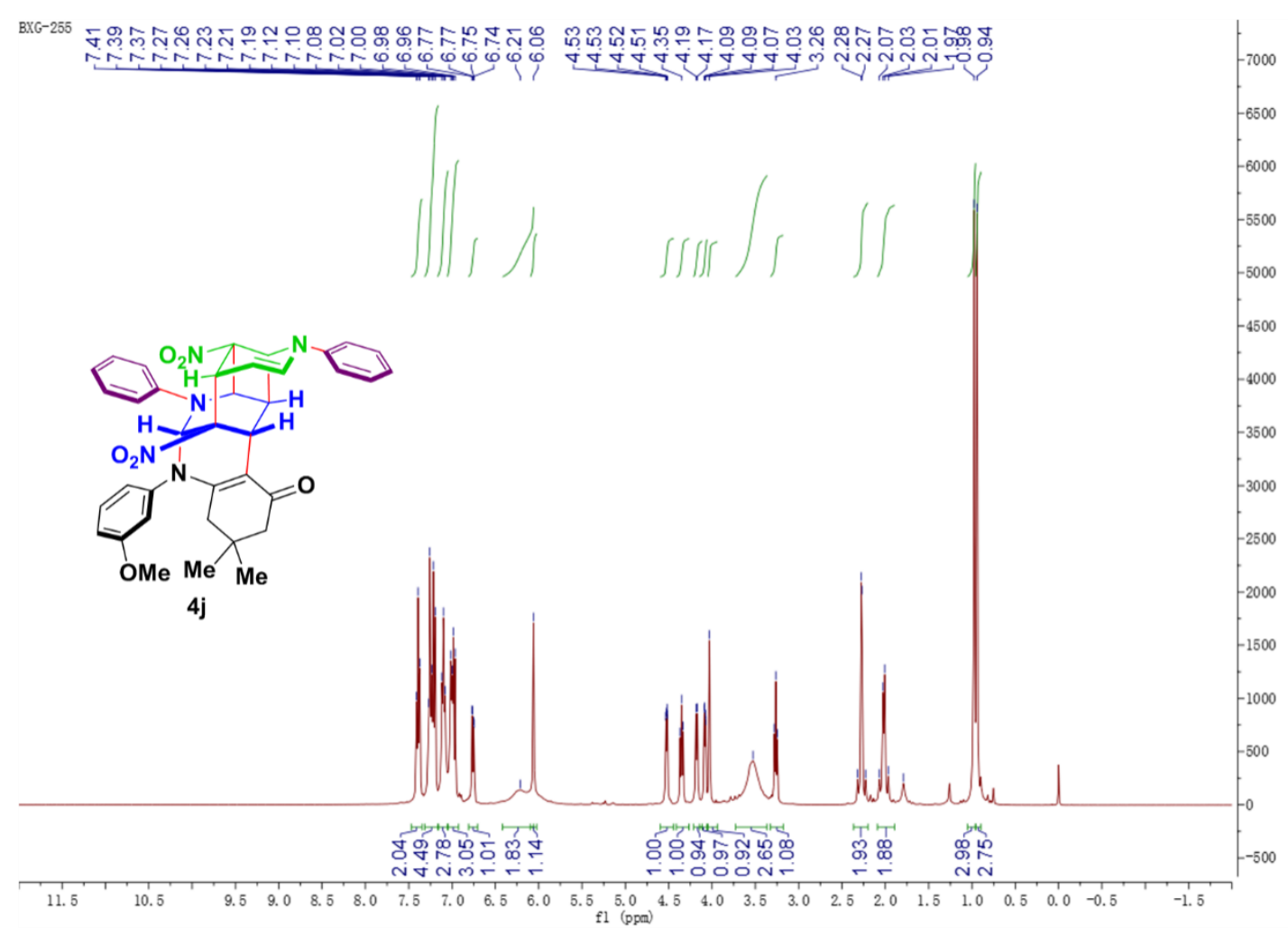

${ }^{13} \mathrm{C}$ NMR spectrum of $\mathbf{4 j}\left(100 \mathrm{MHz}, \mathrm{CDCl}_{3}\right)$ 


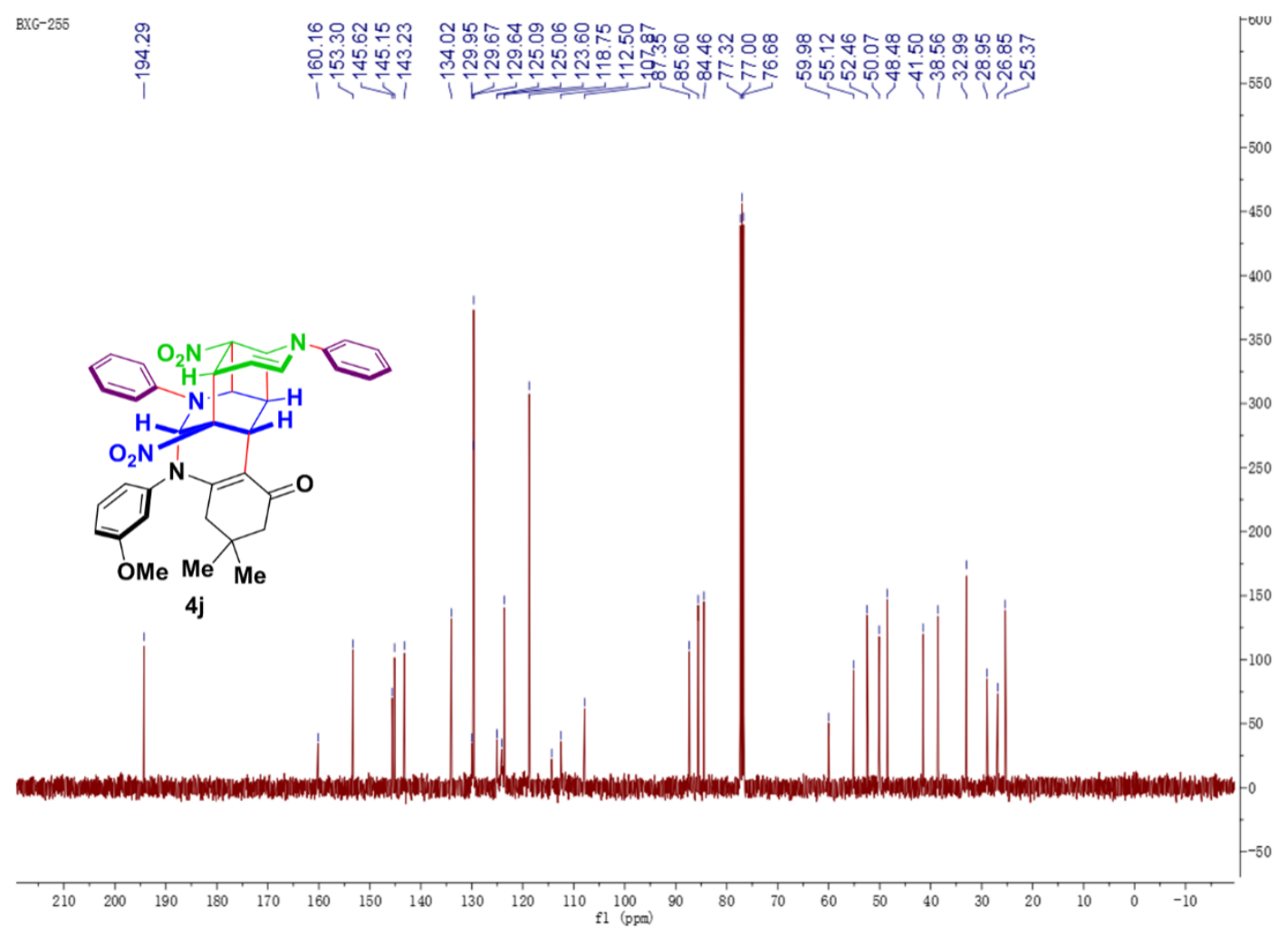

${ }^{1} \mathrm{H}$ NMR spectrum of $\mathbf{4} \mathbf{k}\left(300 \mathrm{MHz}, \mathrm{CDCl}_{3}\right)$

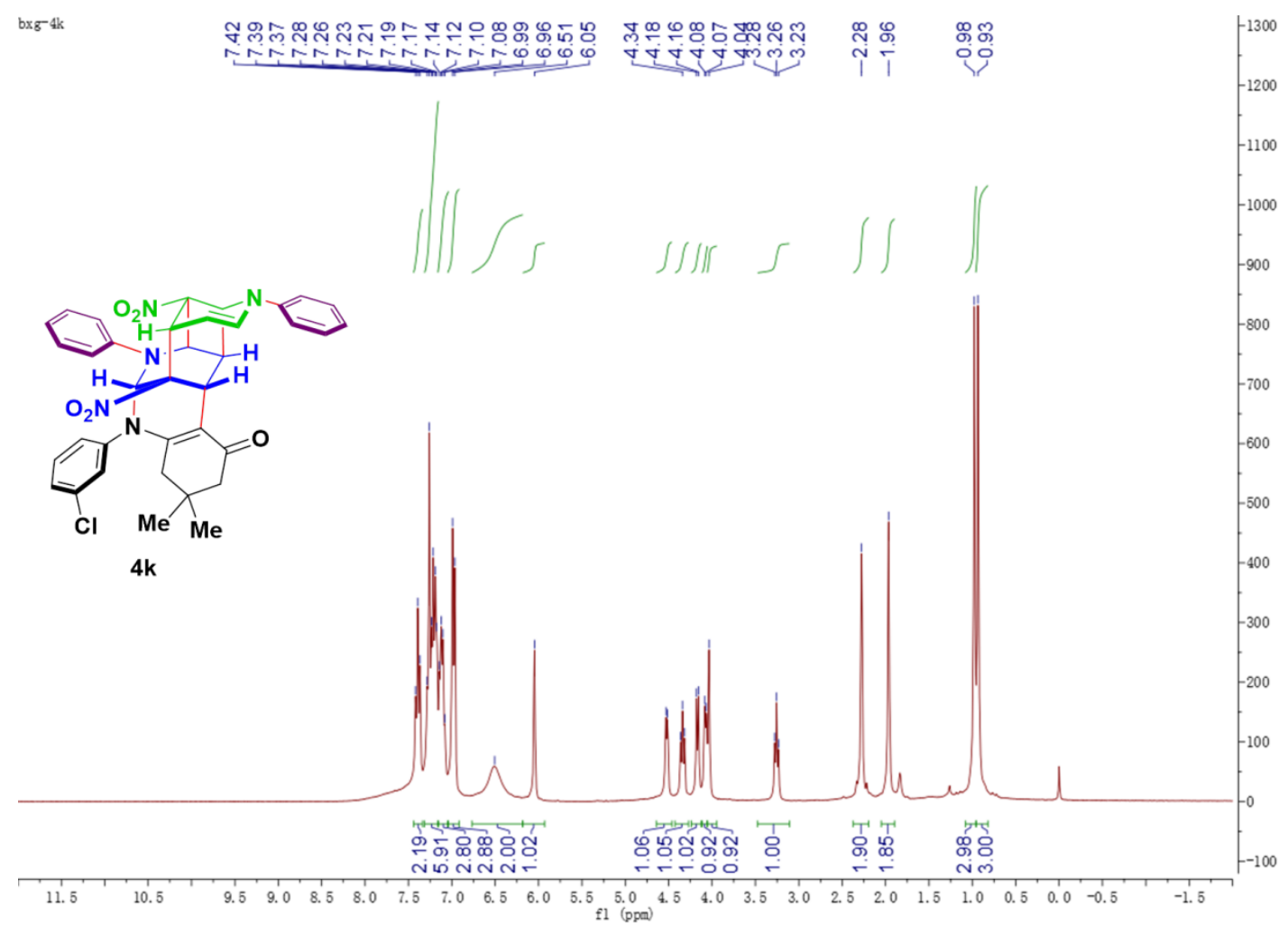

${ }^{13} \mathrm{C}$ NMR spectrum of $4 \mathbf{k}\left(75 \mathrm{MHz}, \mathrm{CDCl}_{3}\right)$ 


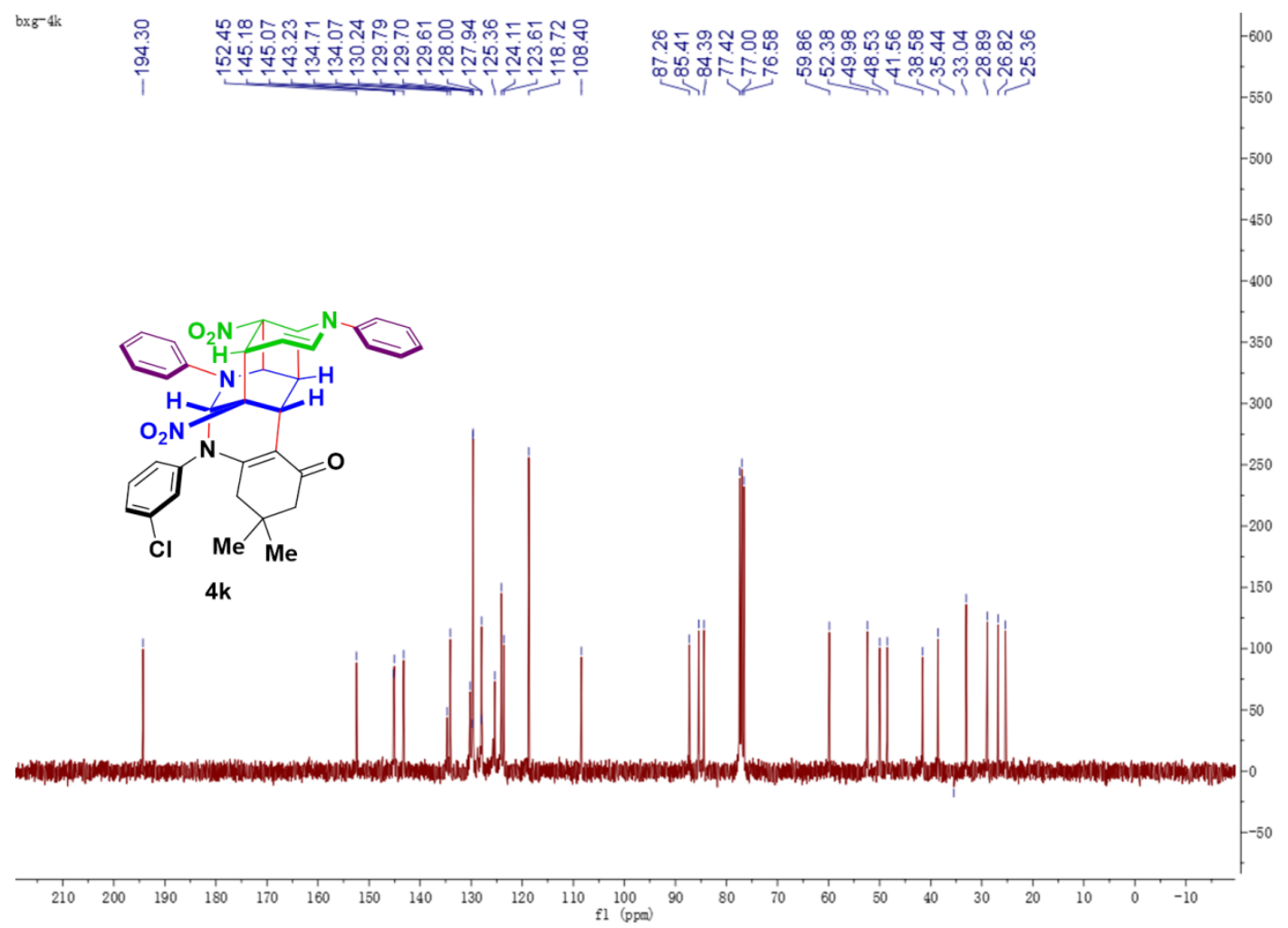

${ }^{1} \mathrm{H}$ NMR spectrum of $4 \mathbf{l}\left(400 \mathrm{MHz}, \mathrm{CDCl}_{3}\right)$

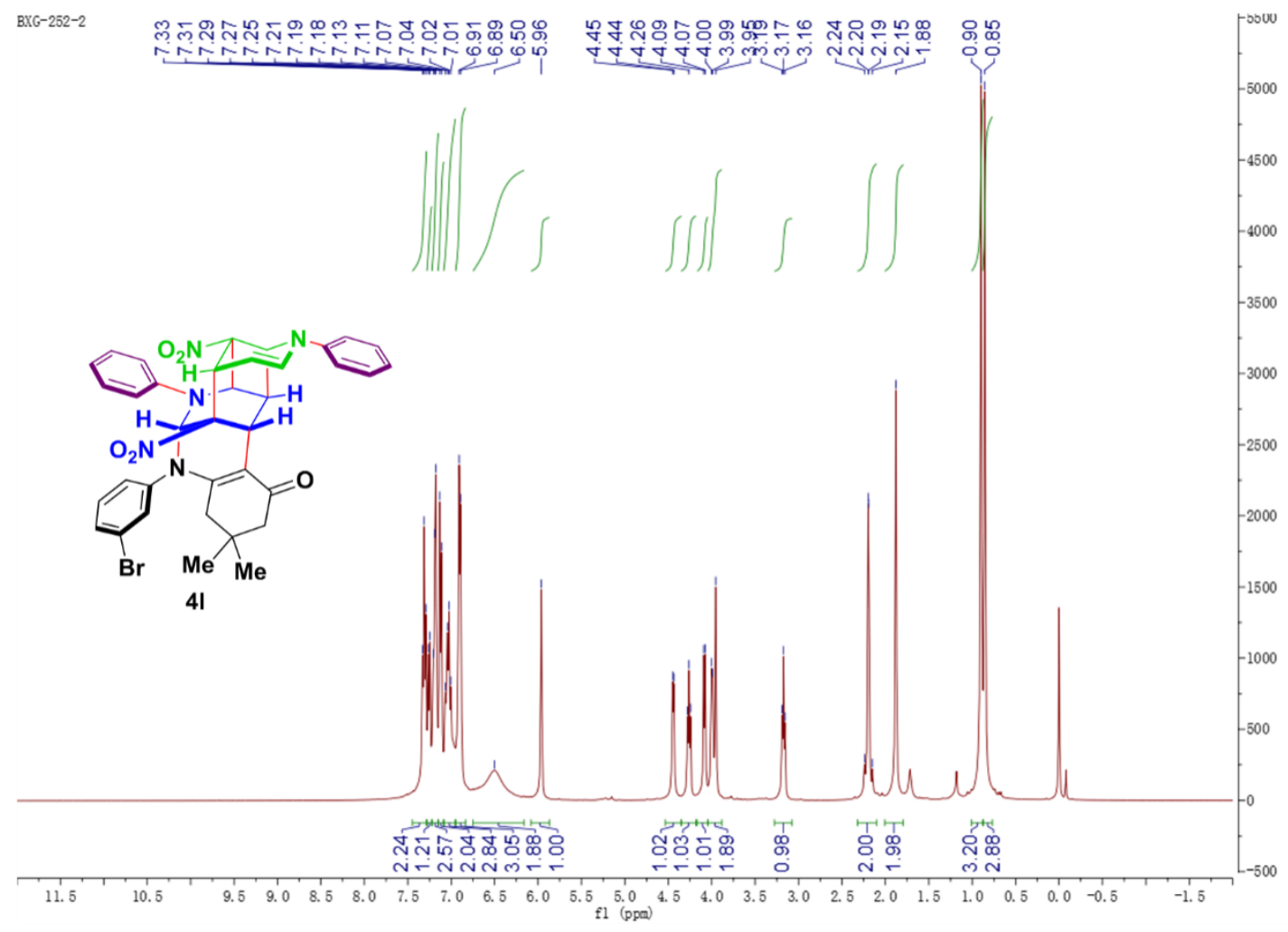

${ }^{13} \mathrm{C}$ NMR spectrum of $4 \mathbf{l}\left(100 \mathrm{MHz}, \mathrm{CDCl}_{3}\right)$ 


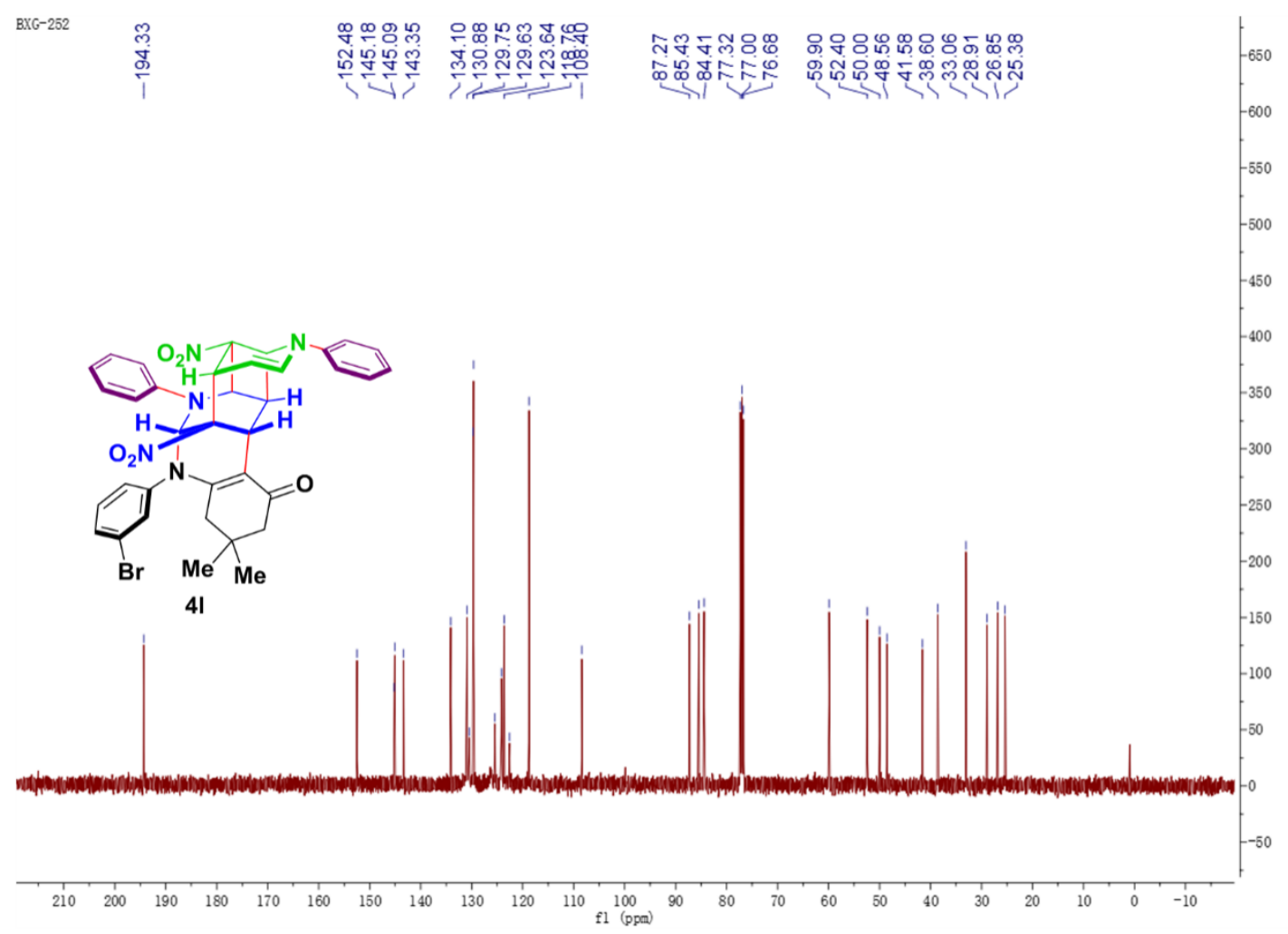

${ }^{1} \mathrm{H}$ NMR spectrum of $\mathbf{4 m}\left(400 \mathrm{MHz}, \mathrm{CDCl}_{3}\right)$

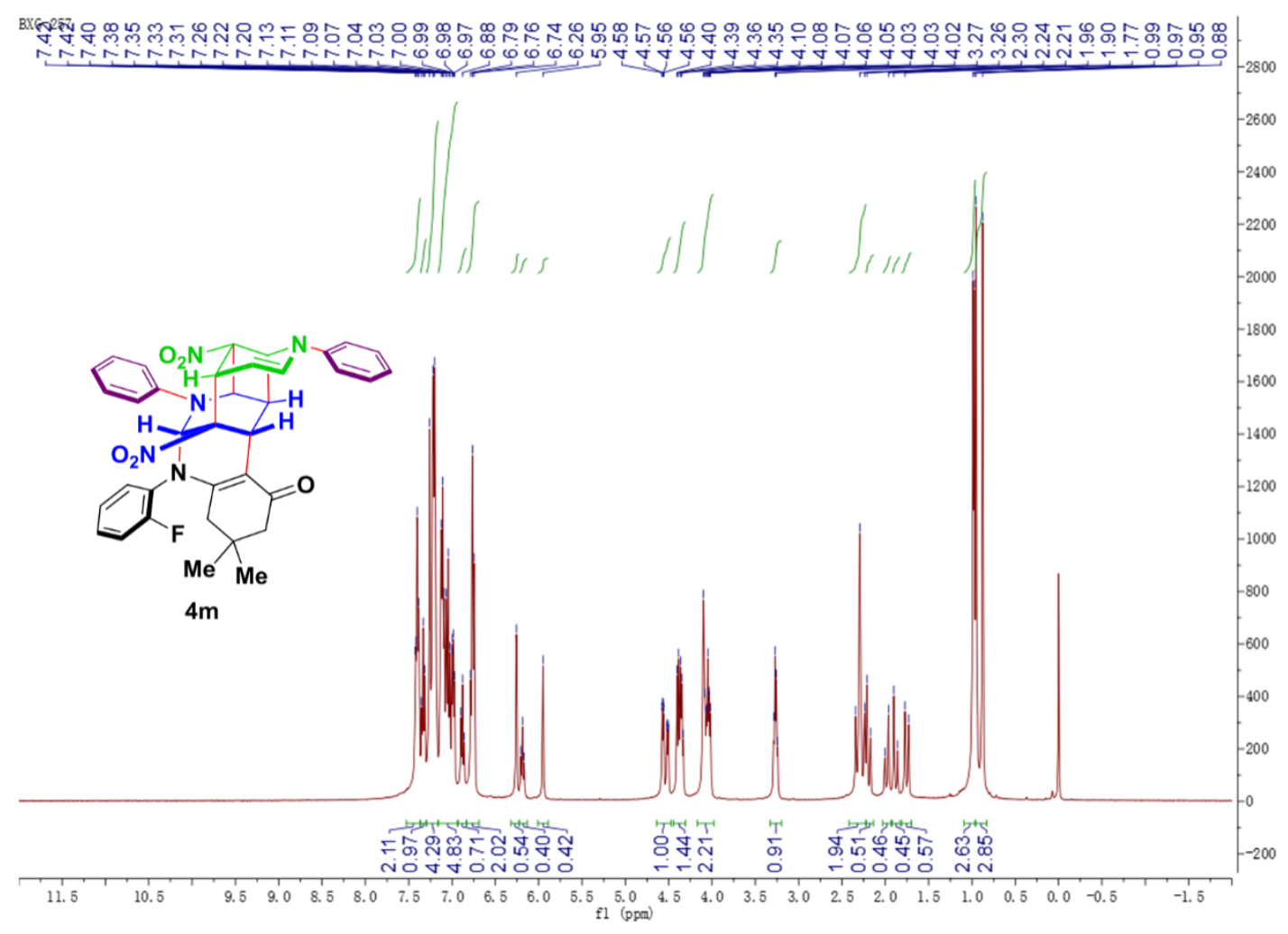

${ }^{13} \mathrm{C}$ NMR spectrum of $\mathbf{4 m}\left(100 \mathrm{MHz}, \mathrm{CDCl}_{3}\right)$ 


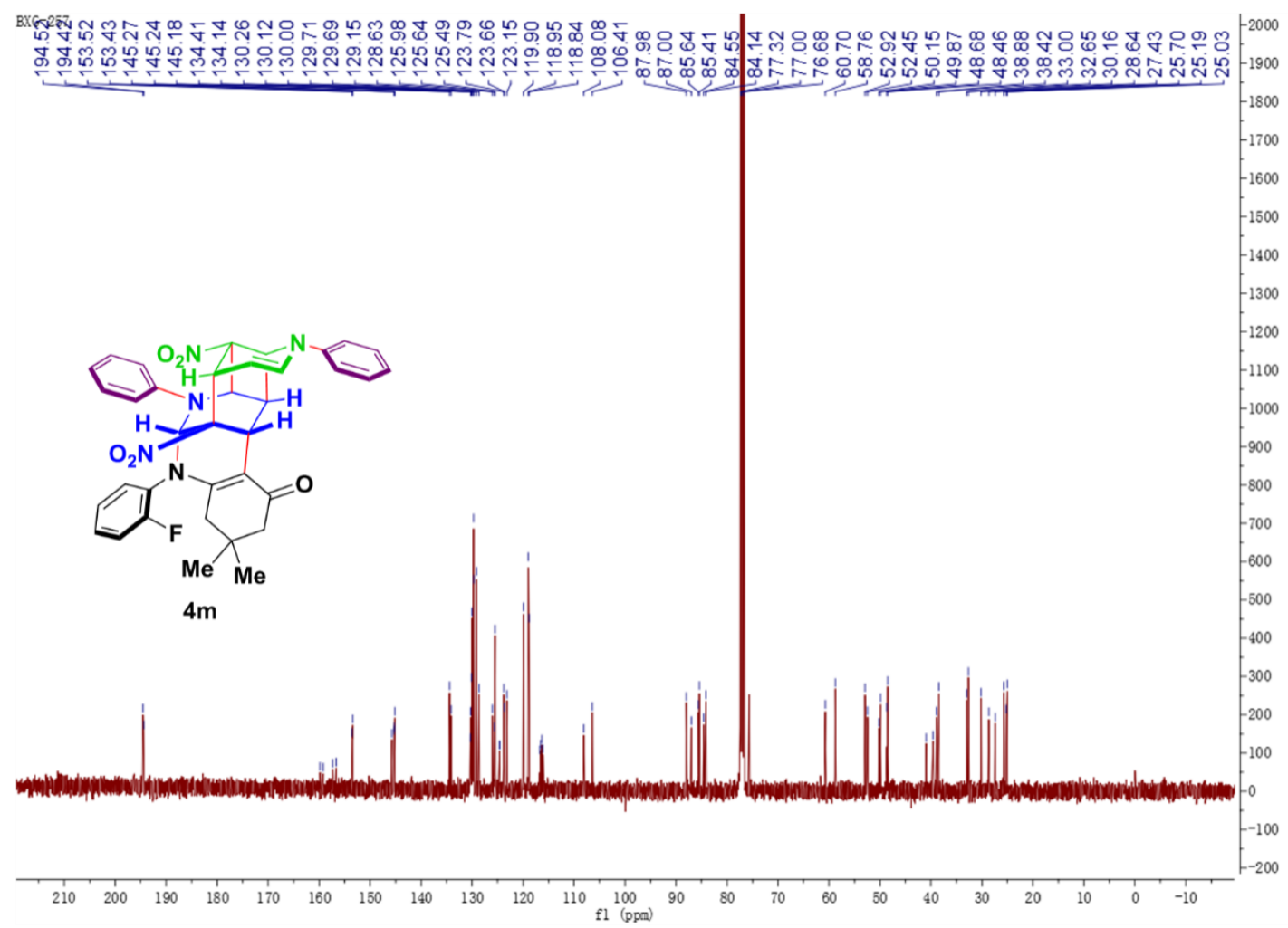

${ }^{1} \mathrm{H}$ NMR spectrum of $\mathbf{4 n}\left(400 \mathrm{MHz}, \mathrm{CDCl}_{3}\right)$

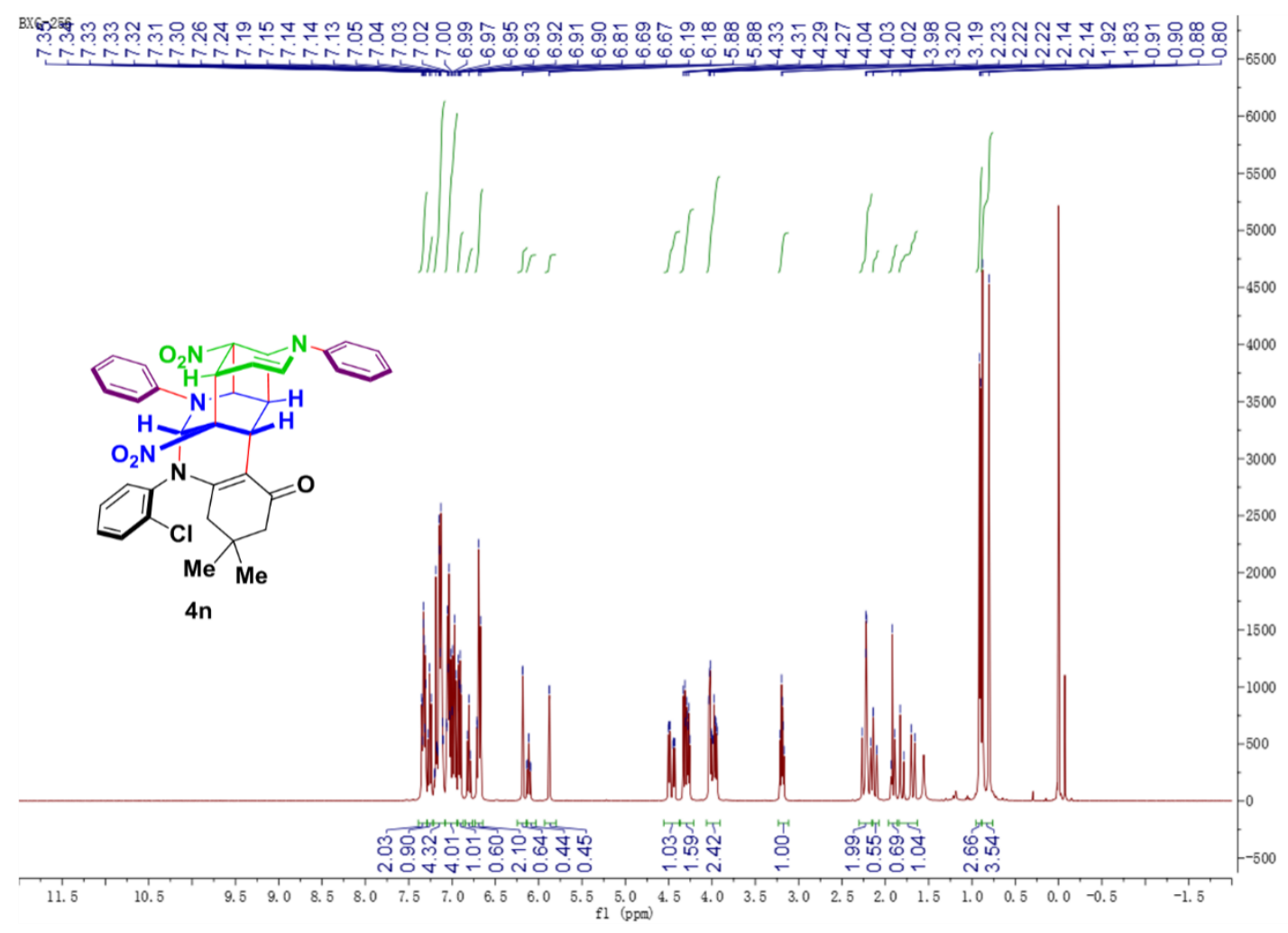

${ }^{13} \mathrm{C}$ NMR spectrum of $4 \mathbf{n}\left(100 \mathrm{MHz}, \mathrm{CDCl}_{3}\right)$ 


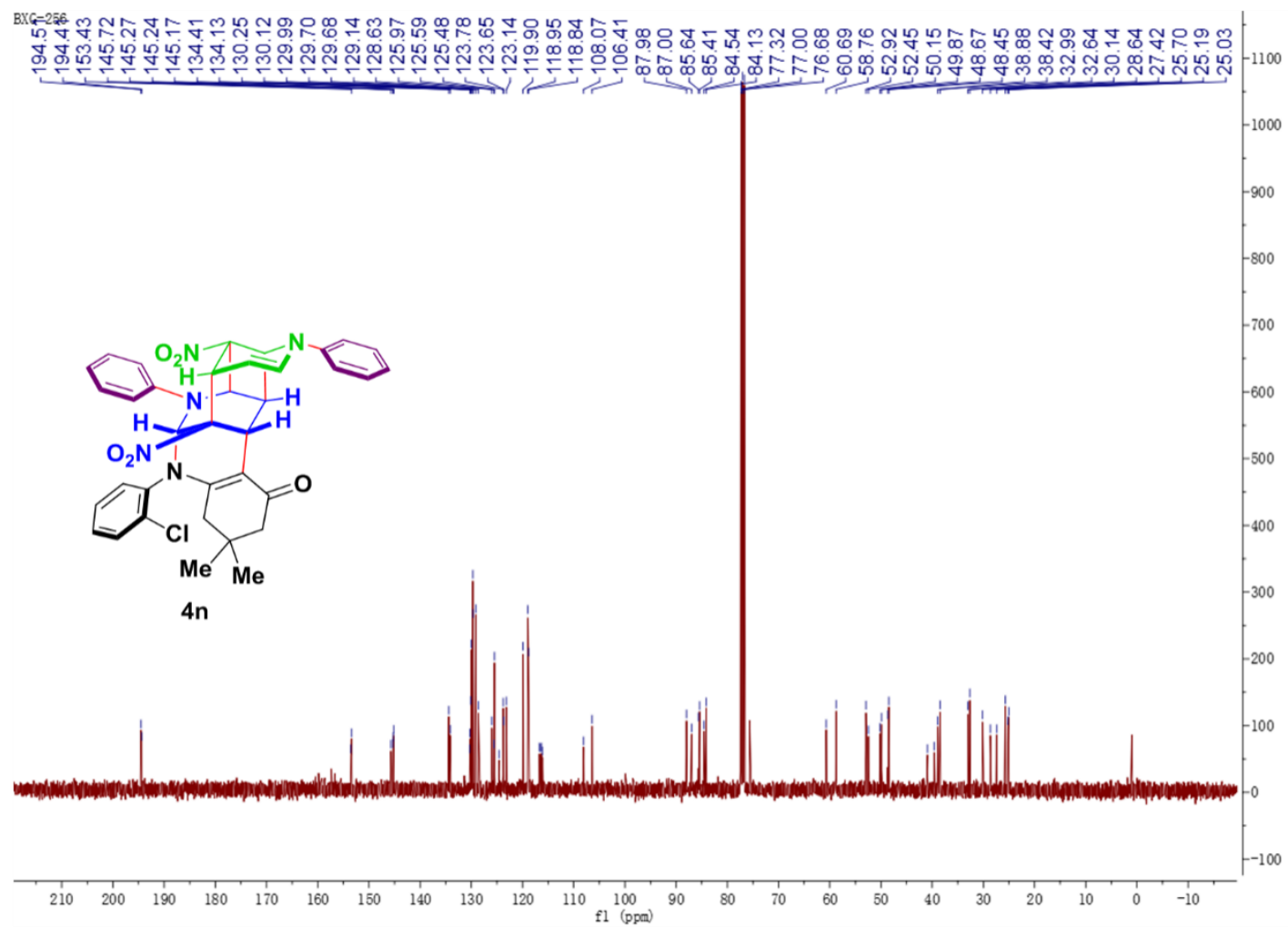

${ }^{1} \mathrm{H}$ NMR spectrum of $40\left(400 \mathrm{MHz}, \mathrm{CDCl}_{3}\right)$

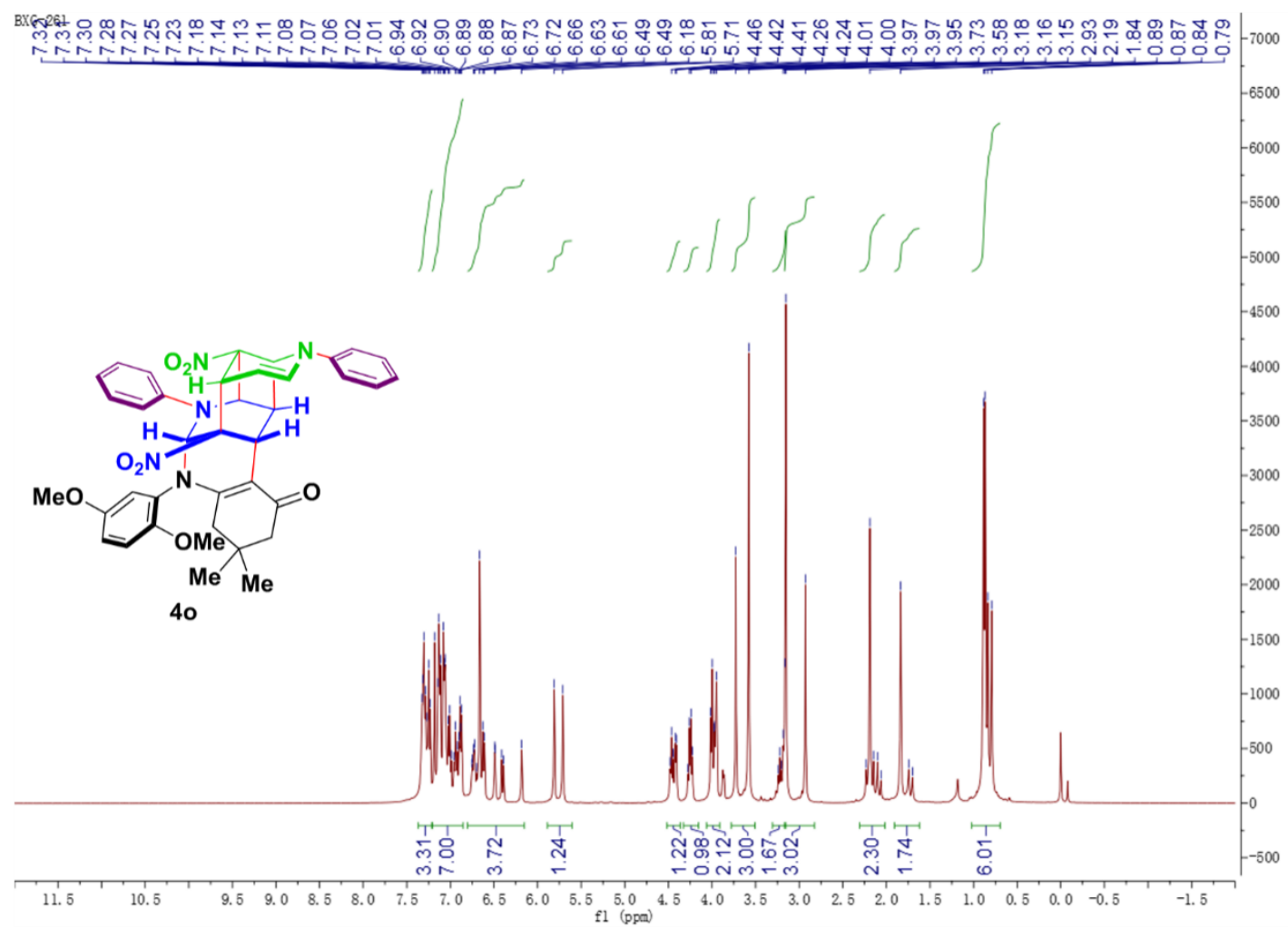

${ }^{13} \mathrm{C}$ NMR spectrum of $4 \mathbf{4 o}\left(100 \mathrm{MHz}, \mathrm{CDCl}_{3}\right)$ 


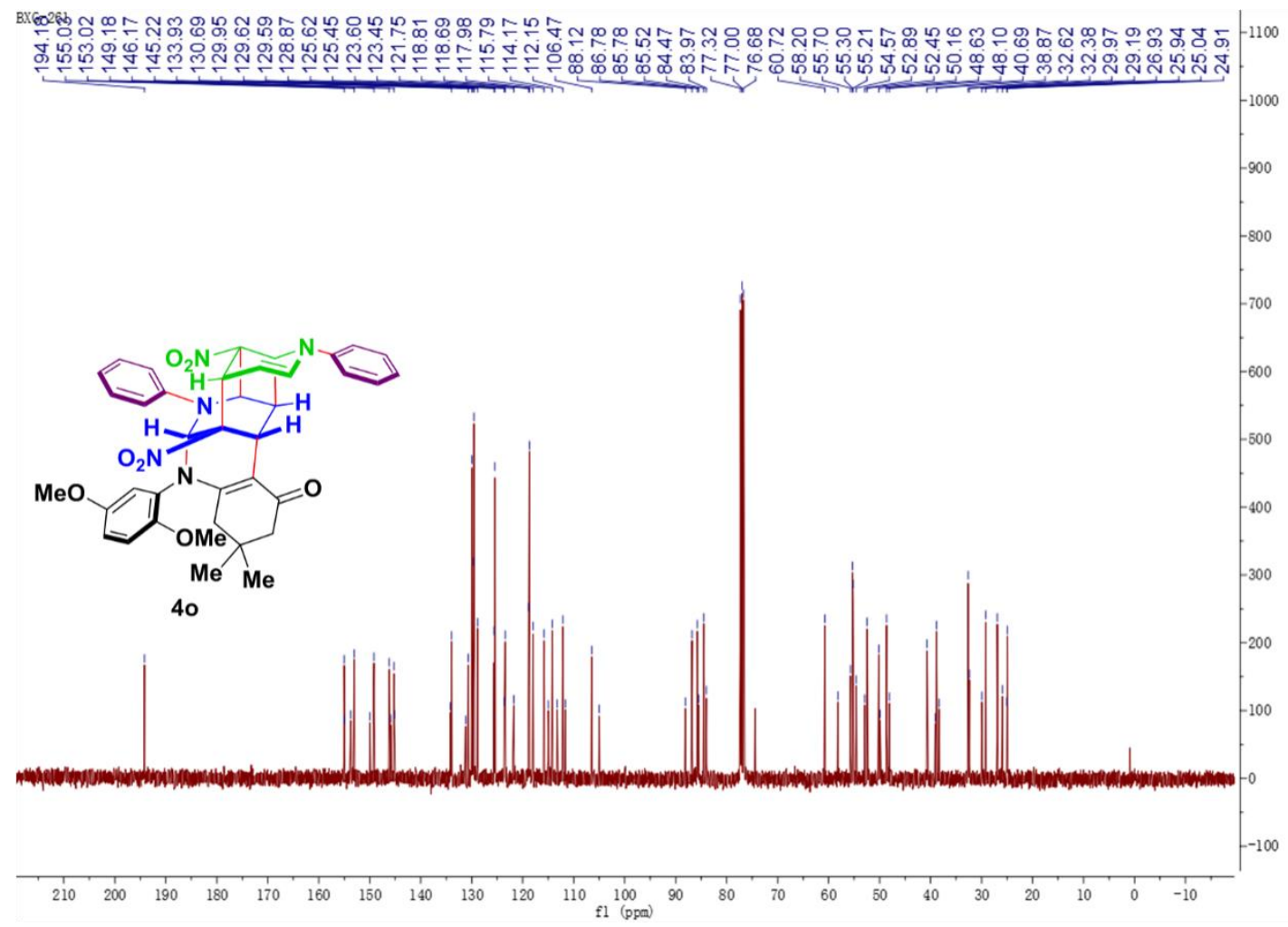

${ }^{1} \mathrm{H}$ NMR spectrum of $\mathbf{4 p}\left(400 \mathrm{MHz}, \mathrm{CDCl}_{3}\right)$

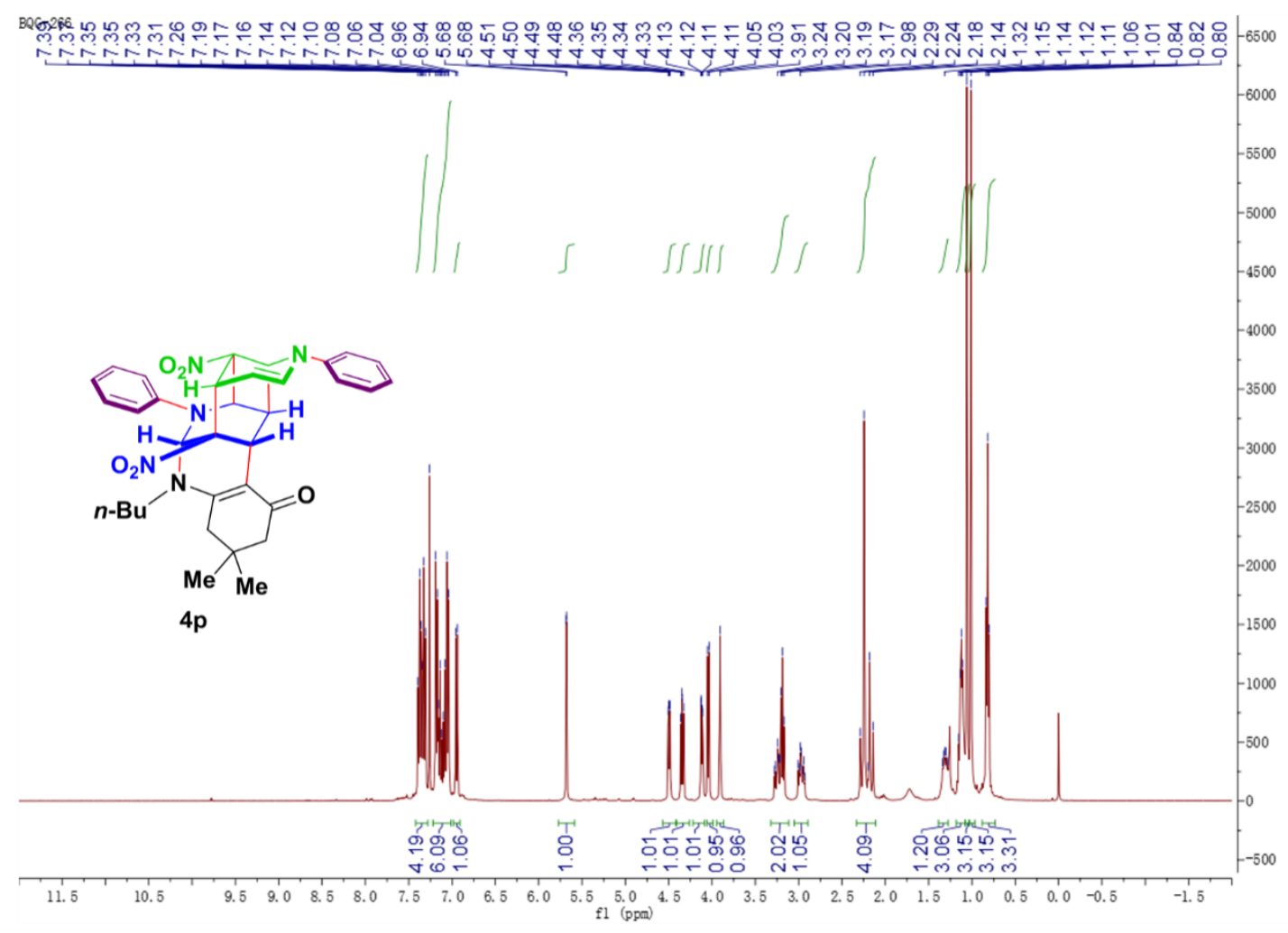

${ }^{13} \mathrm{C}$ NMR spectrum of $\mathbf{4 p}\left(100 \mathrm{MHz}, \mathrm{CDCl}_{3}\right)$ 


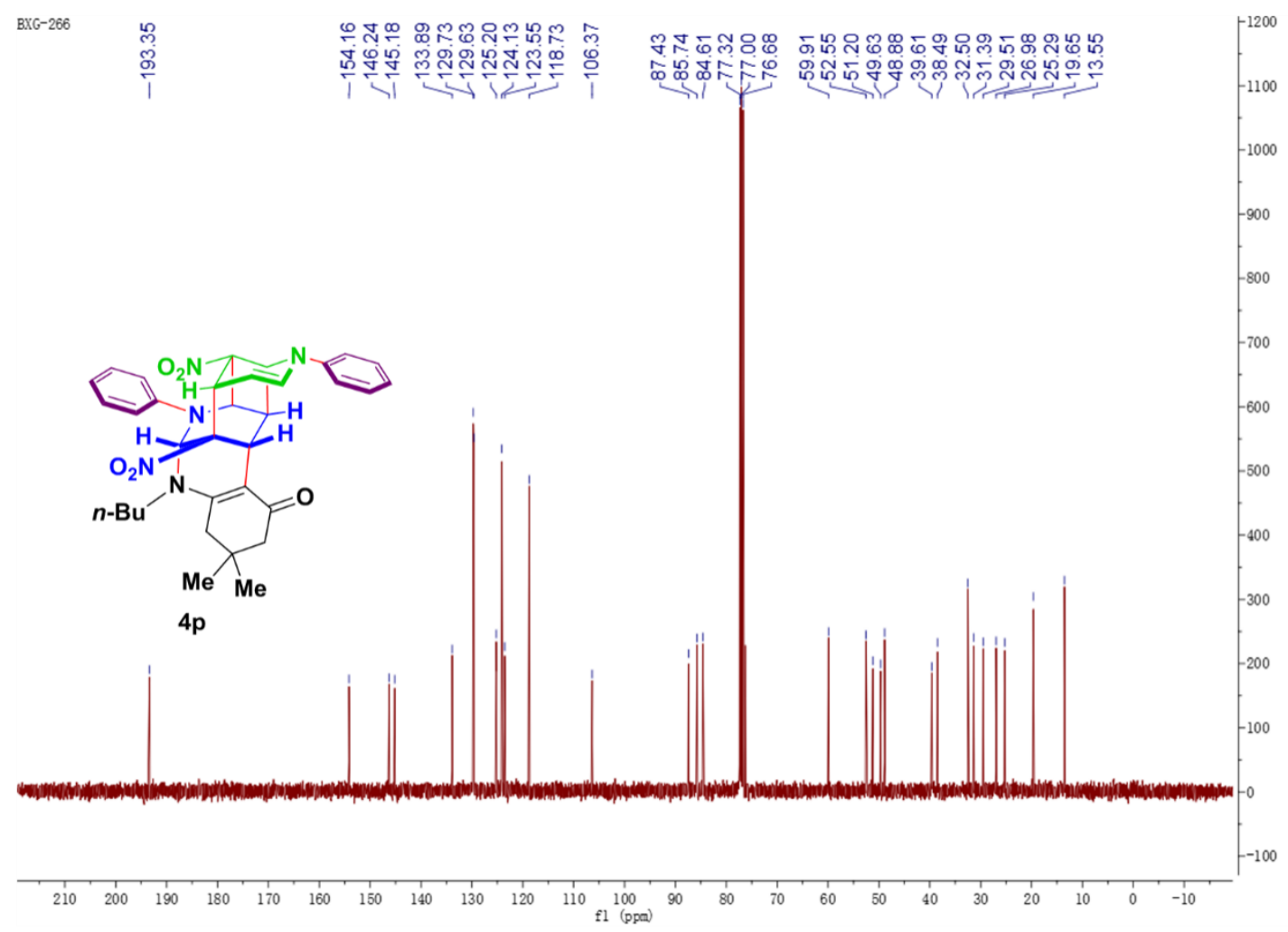

${ }^{1} \mathrm{H}$ NMR spectrum of $\mathbf{4 q}\left(400 \mathrm{MHz}, \mathrm{CDCl}_{3}\right)$

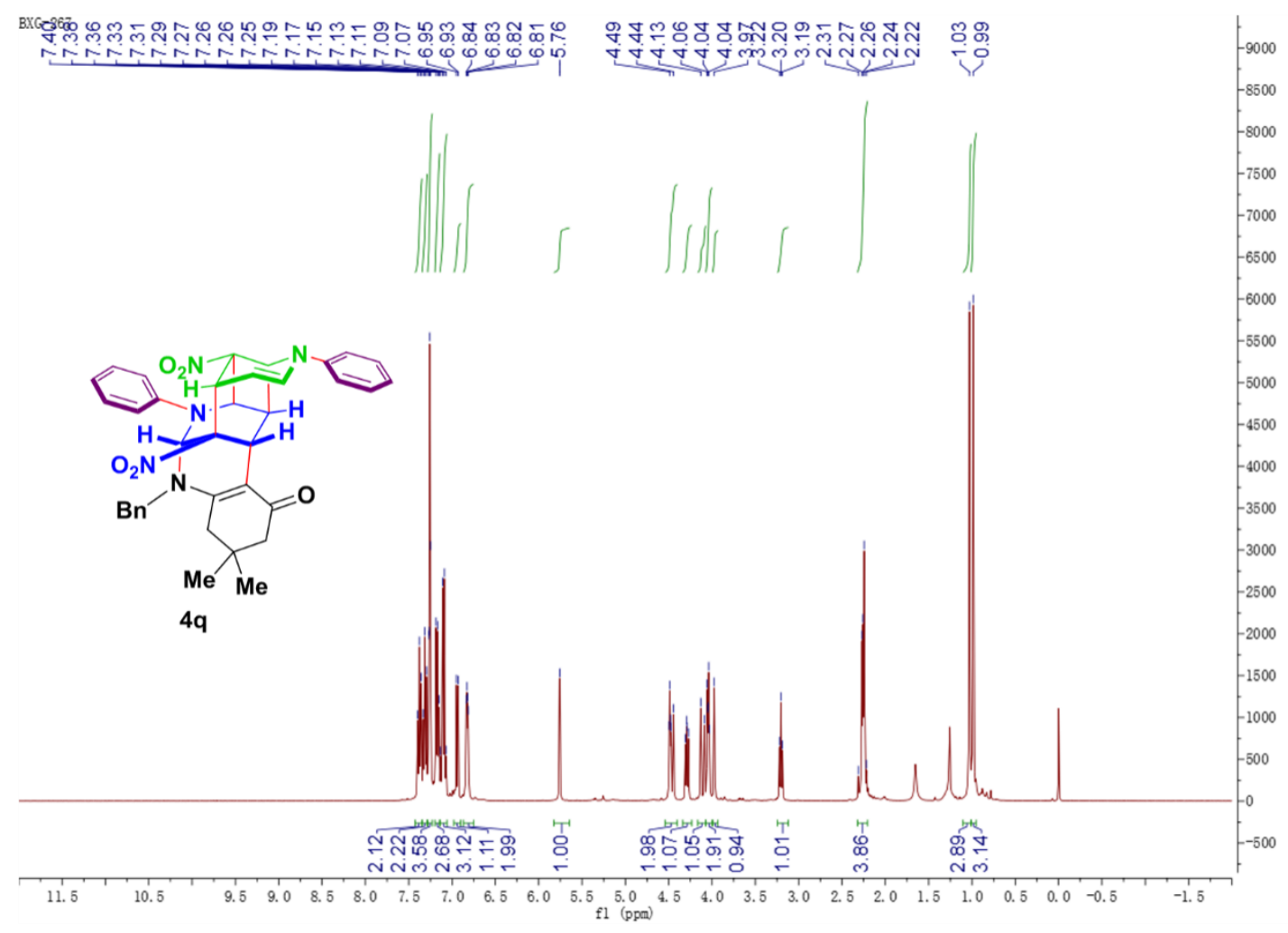

${ }^{13} \mathrm{C}$ NMR spectrum of $\mathbf{4 q}\left(100 \mathrm{MHz}, \mathrm{CDCl}_{3}\right)$ 


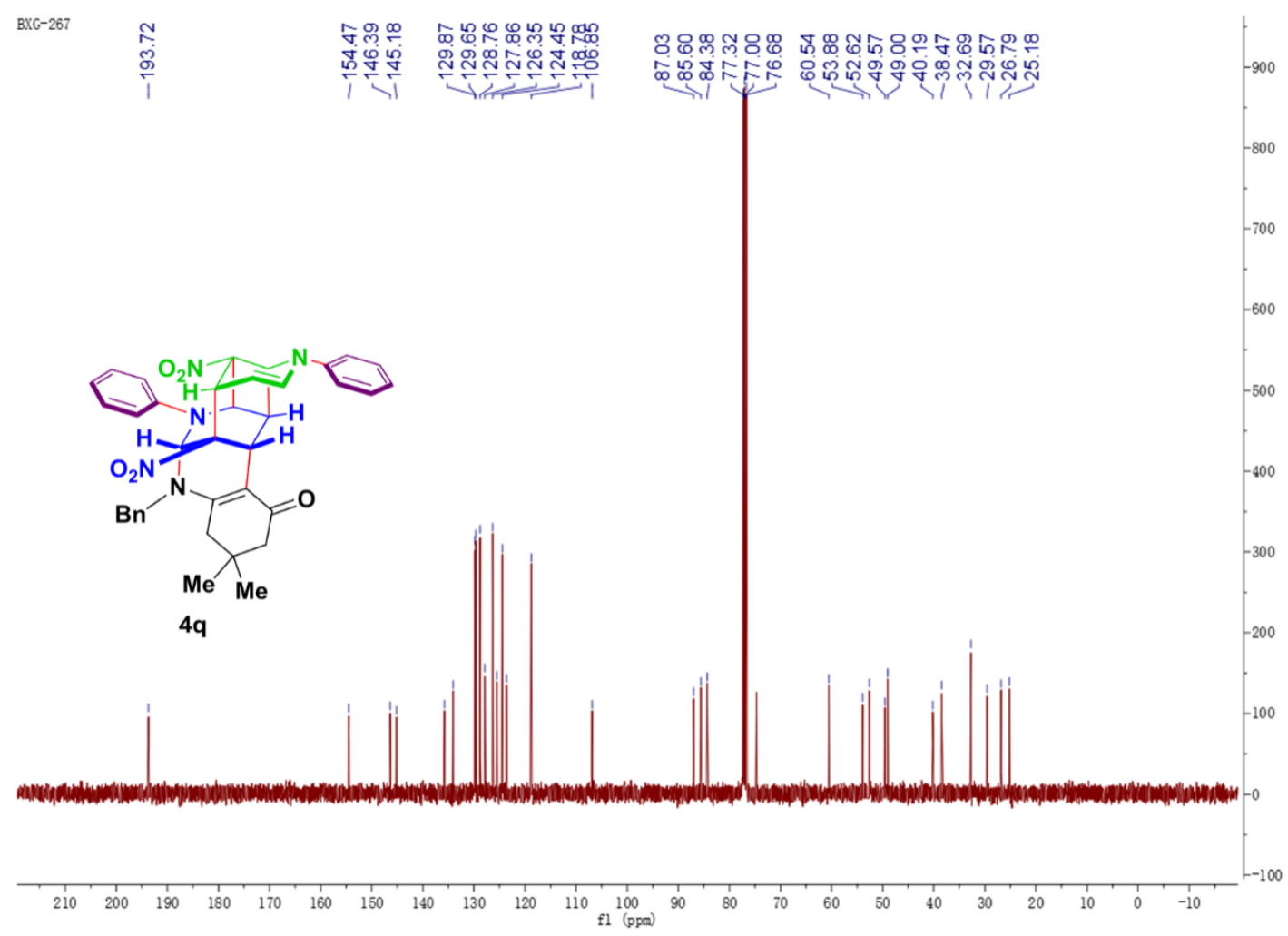

${ }^{1} \mathrm{H}$ NMR spectrum of $\mathbf{4 r}\left(400 \mathrm{MHz}, \mathrm{CDCl}_{3}\right)$

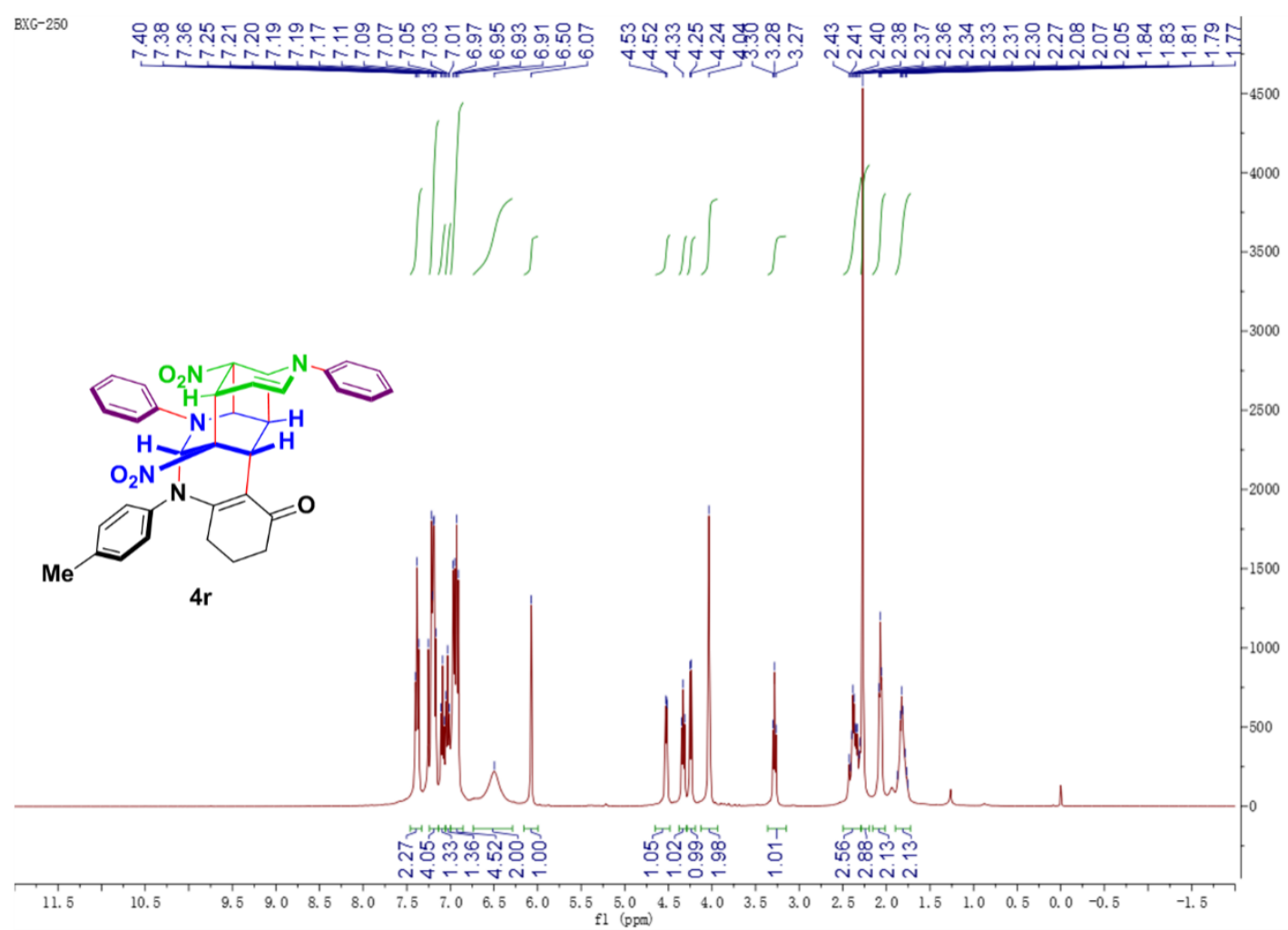

${ }^{13} \mathrm{C}$ NMR spectrum of $4 \mathbf{r}\left(100 \mathrm{MHz}, \mathrm{CDCl}_{3}\right)$ 


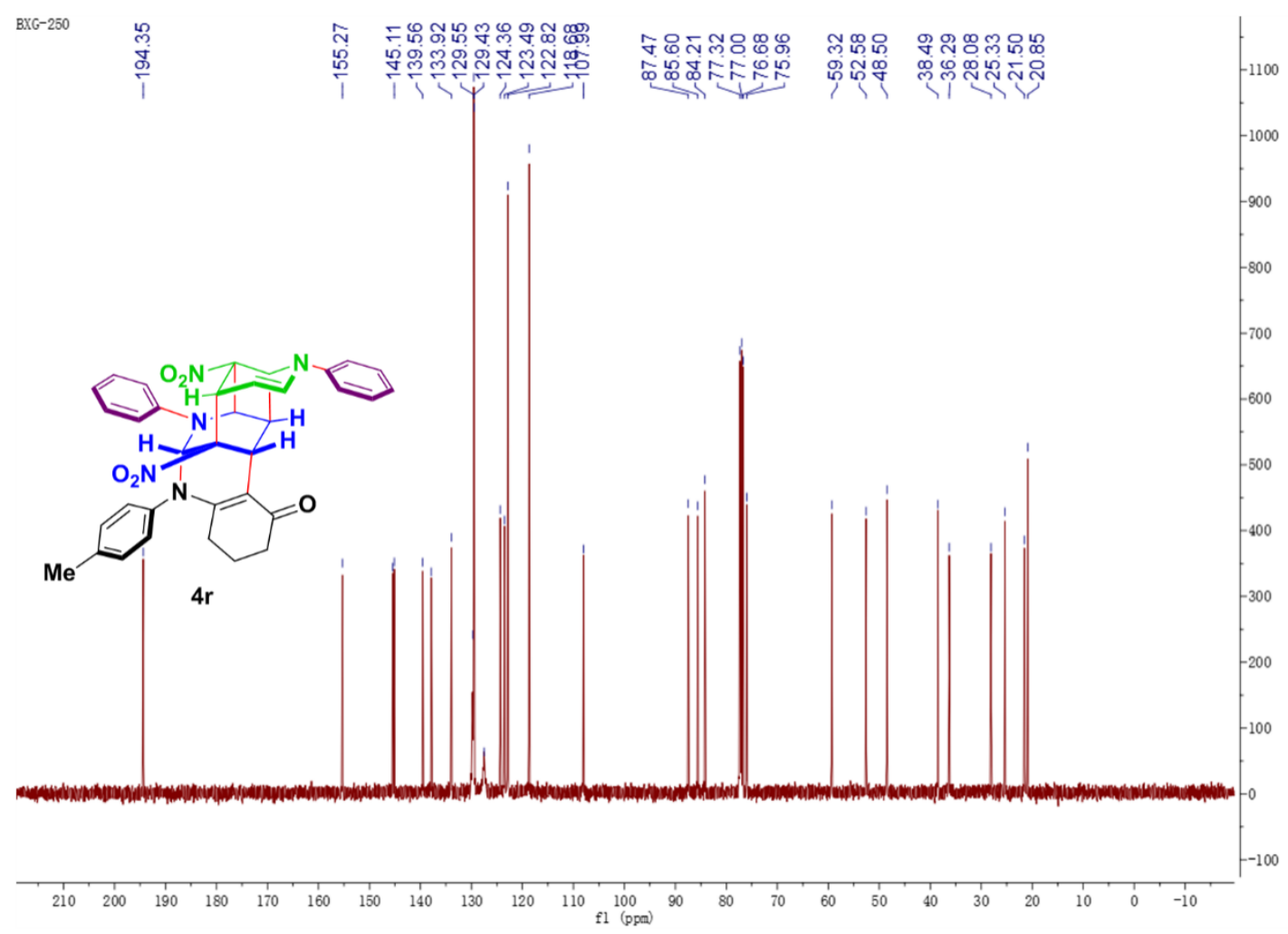

${ }^{1} \mathrm{H}$ NMR spectrum of $4 \mathbf{s}\left(400 \mathrm{MHz}, \mathrm{CDCl}_{3}\right)$

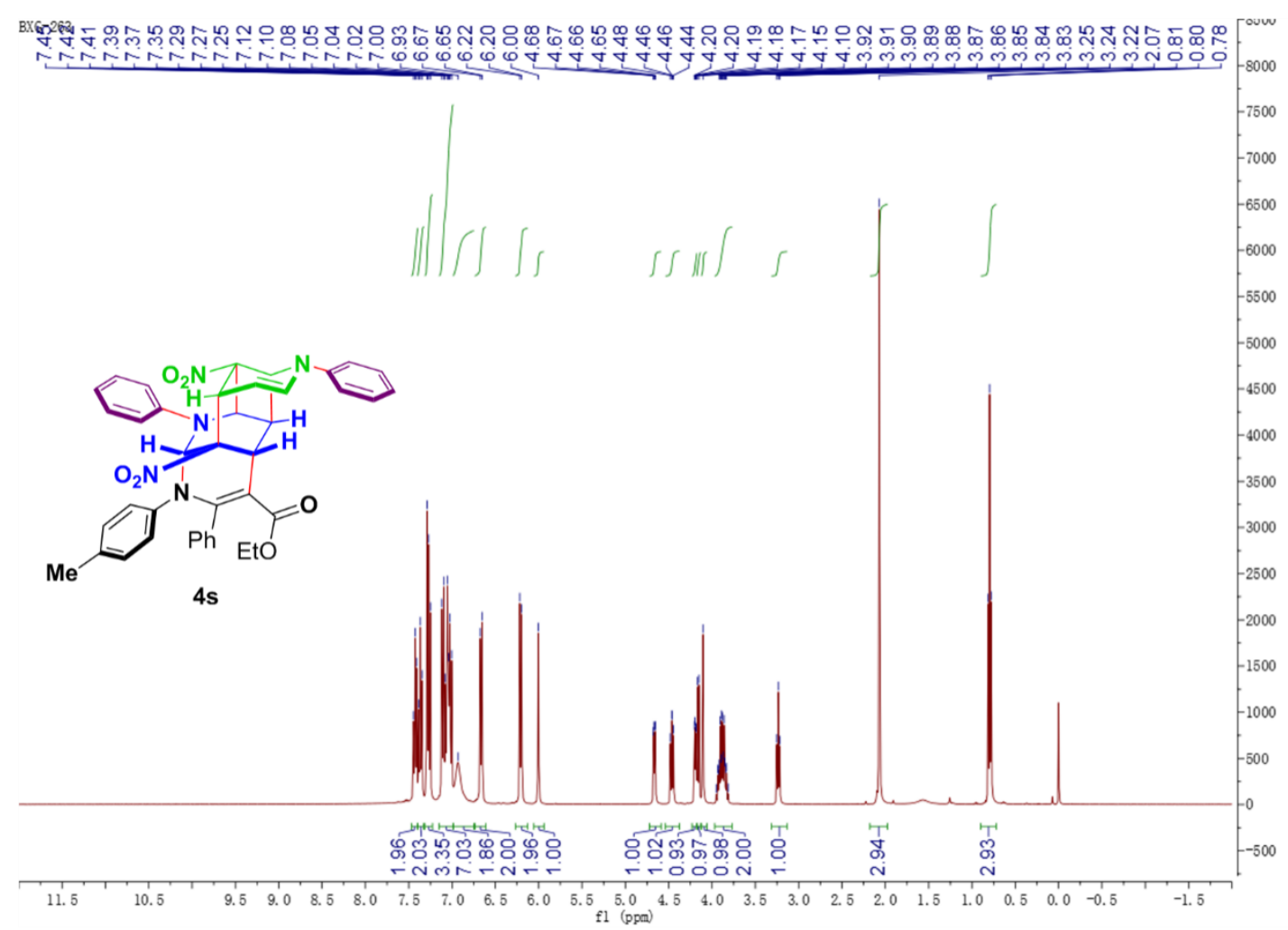

${ }^{13} \mathrm{C}$ NMR spectrum of $4 \mathbf{s}\left(100 \mathrm{MHz}, \mathrm{CDCl}_{3}\right)$ 


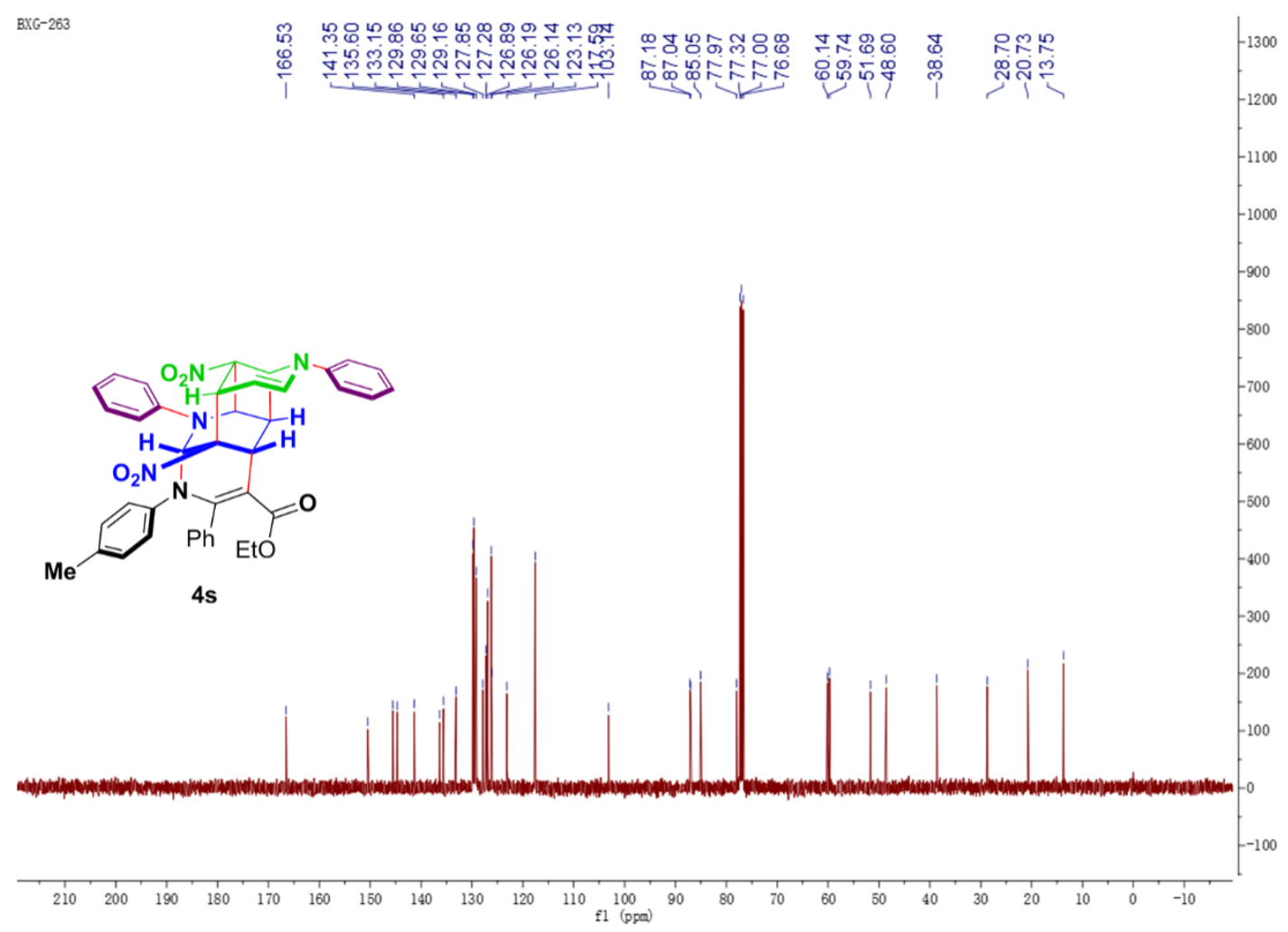

${ }^{1} \mathrm{H}$ NMR spectrum of $\mathbf{4 t}\left(400 \mathrm{MHz}, \mathrm{CDCl}_{3}\right)$

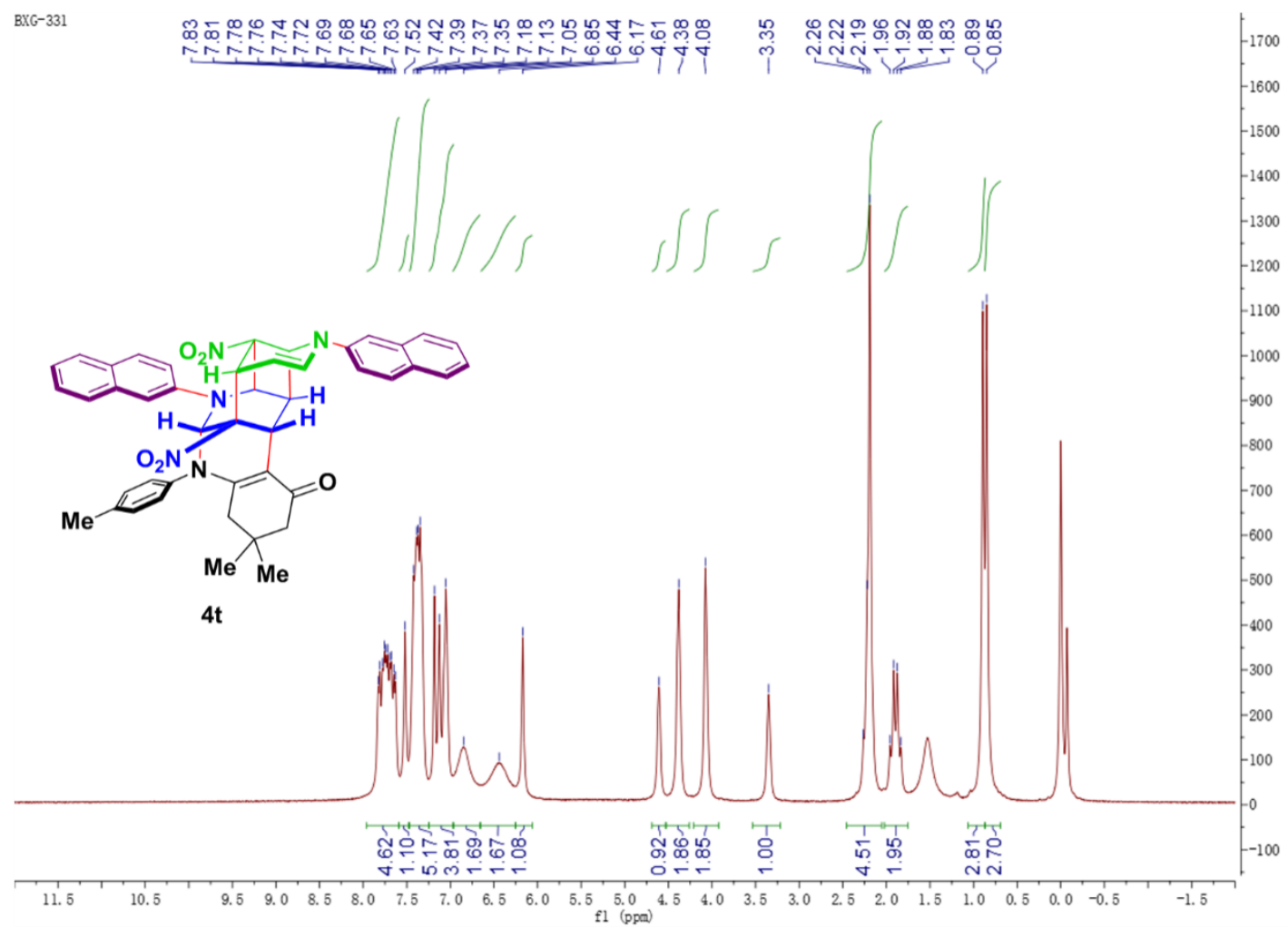

${ }^{13} \mathrm{C}$ NMR spectrum of $\mathbf{4 t}\left(100 \mathrm{MHz}, \mathrm{CDCl}_{3}\right)$ 


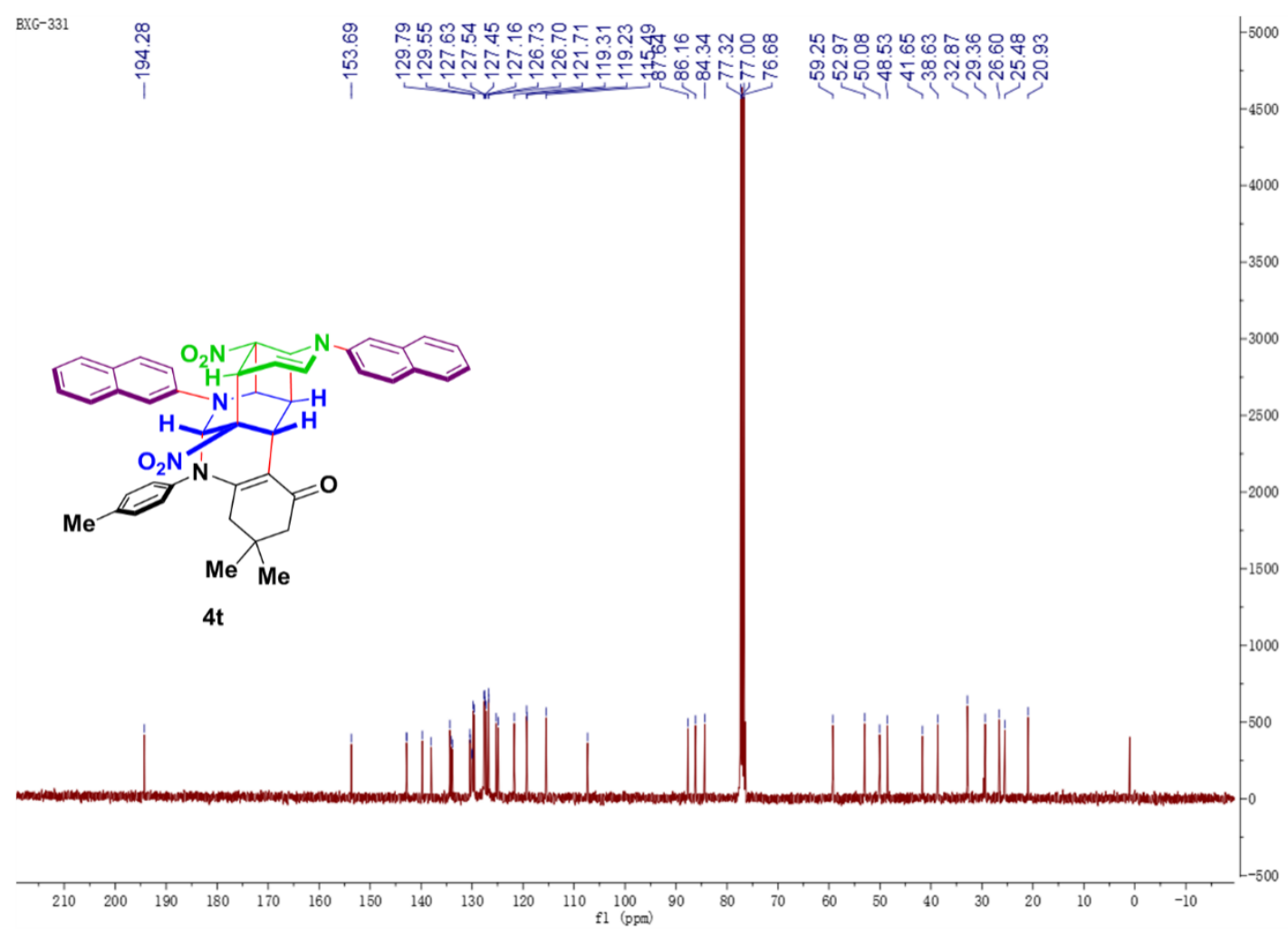

${ }^{1} \mathrm{H}$ NMR spectrum of $\mathbf{4 u}\left(400 \mathrm{MHz}, \mathrm{CDCl}_{3}\right)$

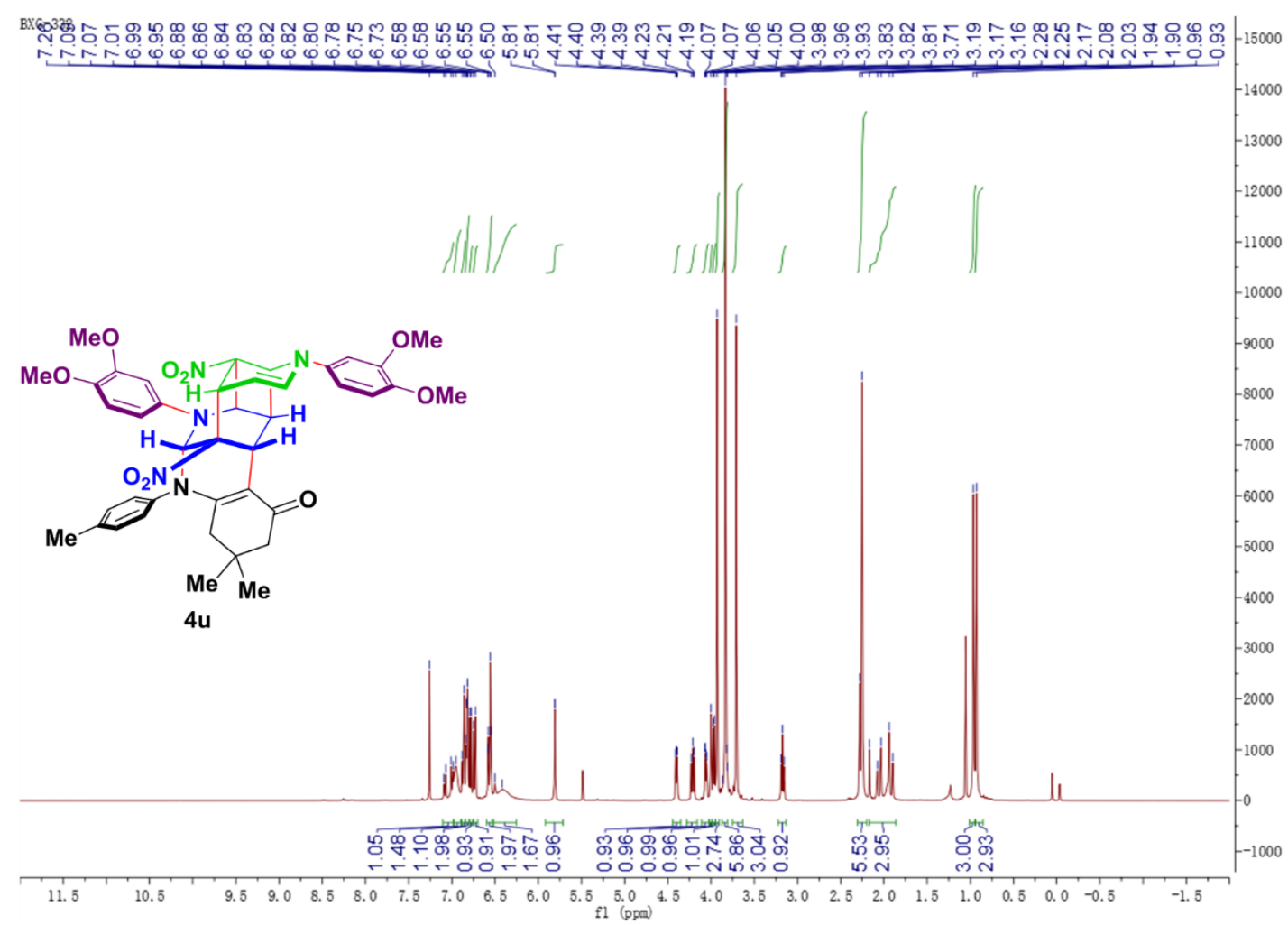

${ }^{13} \mathrm{C}$ NMR spectrum of $\mathbf{4 u}\left(100 \mathrm{MHz}, \mathrm{CDCl}_{3}\right)$ 


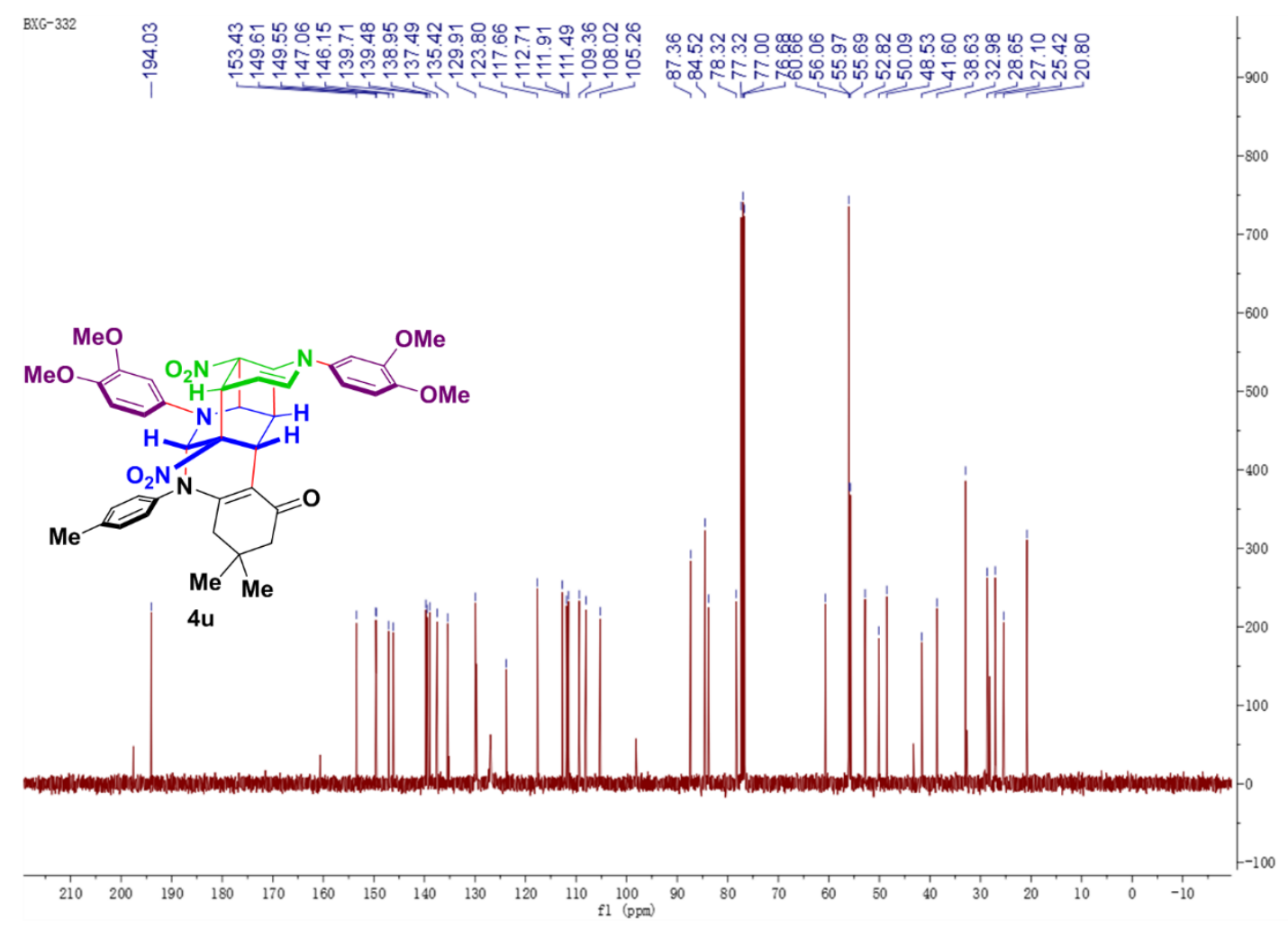

${ }^{1} \mathrm{H}$ NMR spectrum of $\mathbf{6 a}\left(400 \mathrm{MHz}, \mathrm{CDCl}_{3}\right)$

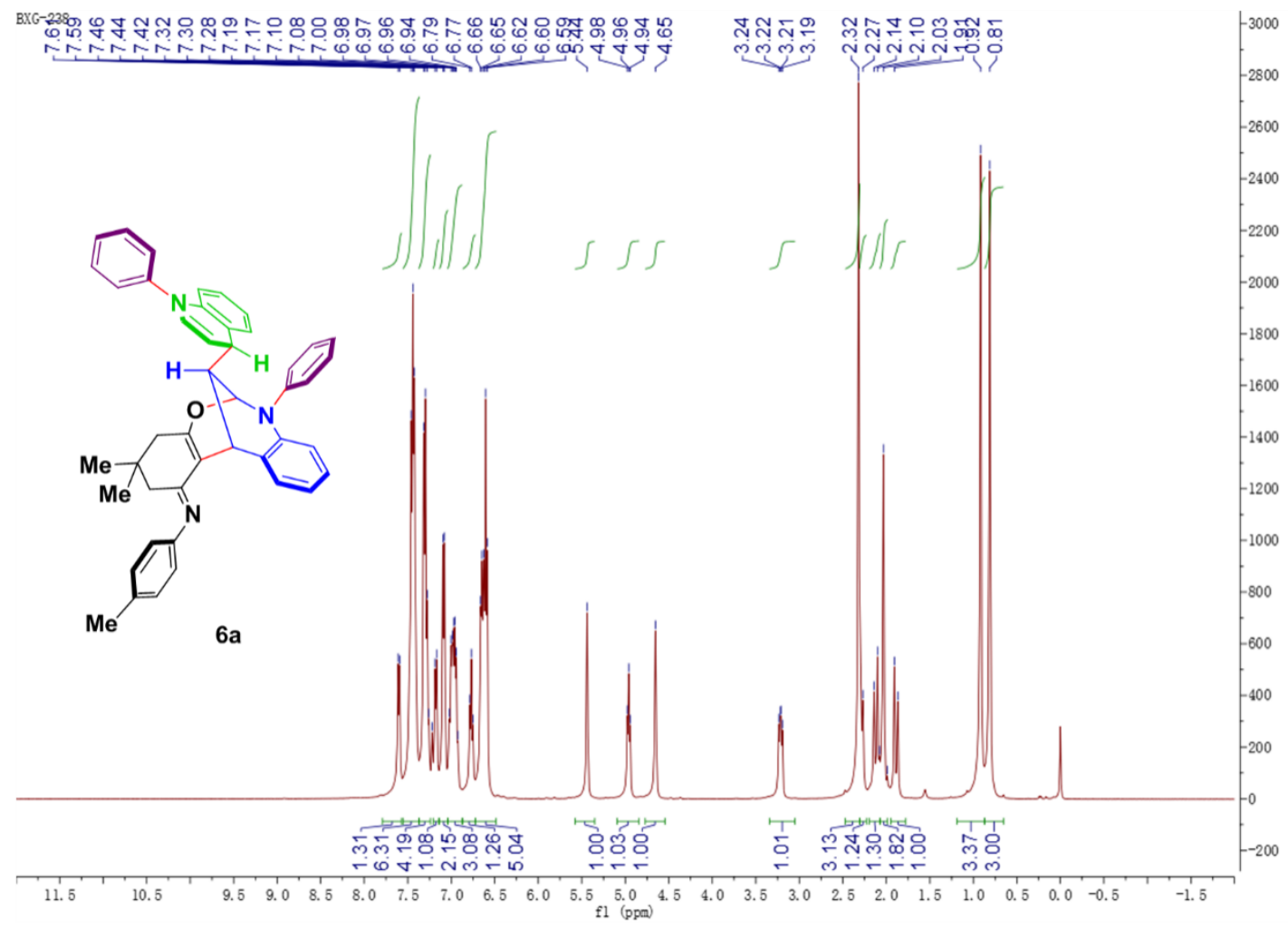

${ }^{13} \mathrm{C}$ NMR spectrum of $\mathbf{6 a}\left(100 \mathrm{MHz}, \mathrm{CDCl}_{3}\right)$ 


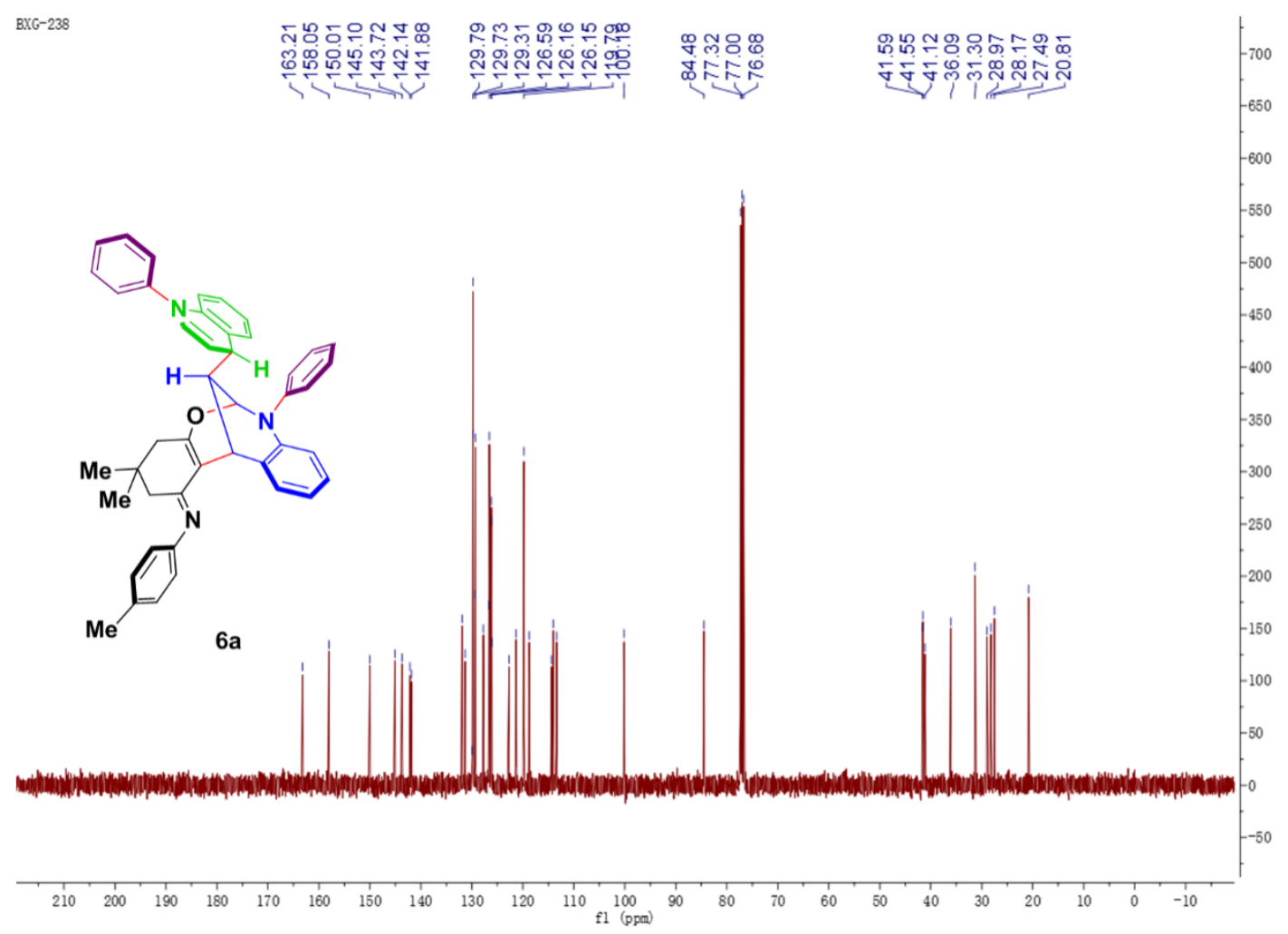

${ }^{1} \mathrm{H}$ NMR spectrum of $\mathbf{6 b}\left(300 \mathrm{MHz}, \mathrm{CDCl}_{3}\right)$

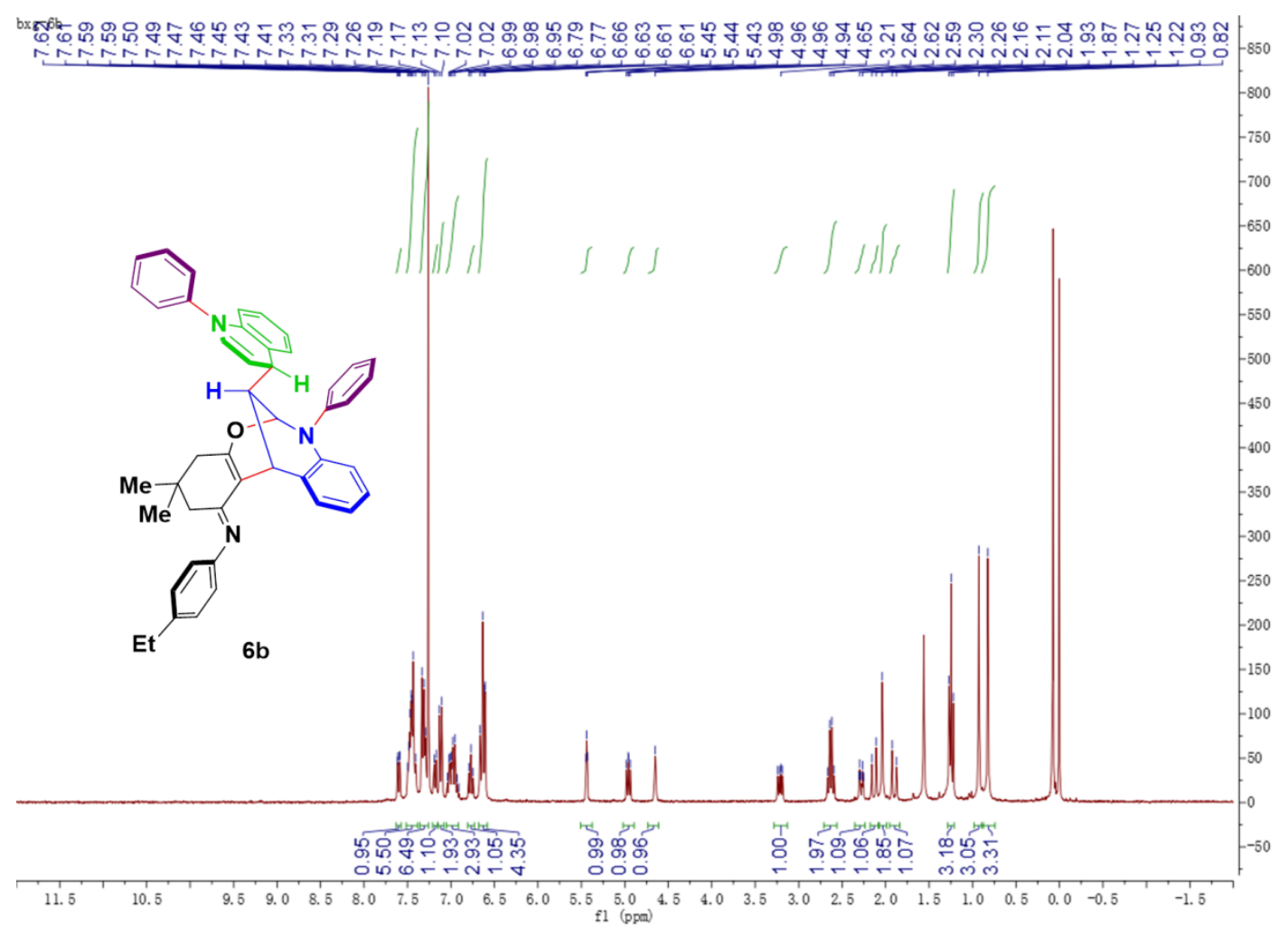

${ }^{13} \mathrm{C}$ NMR spectrum of $6 \mathbf{b}\left(75 \mathrm{MHz}, \mathrm{CDCl}_{3}\right)$ 


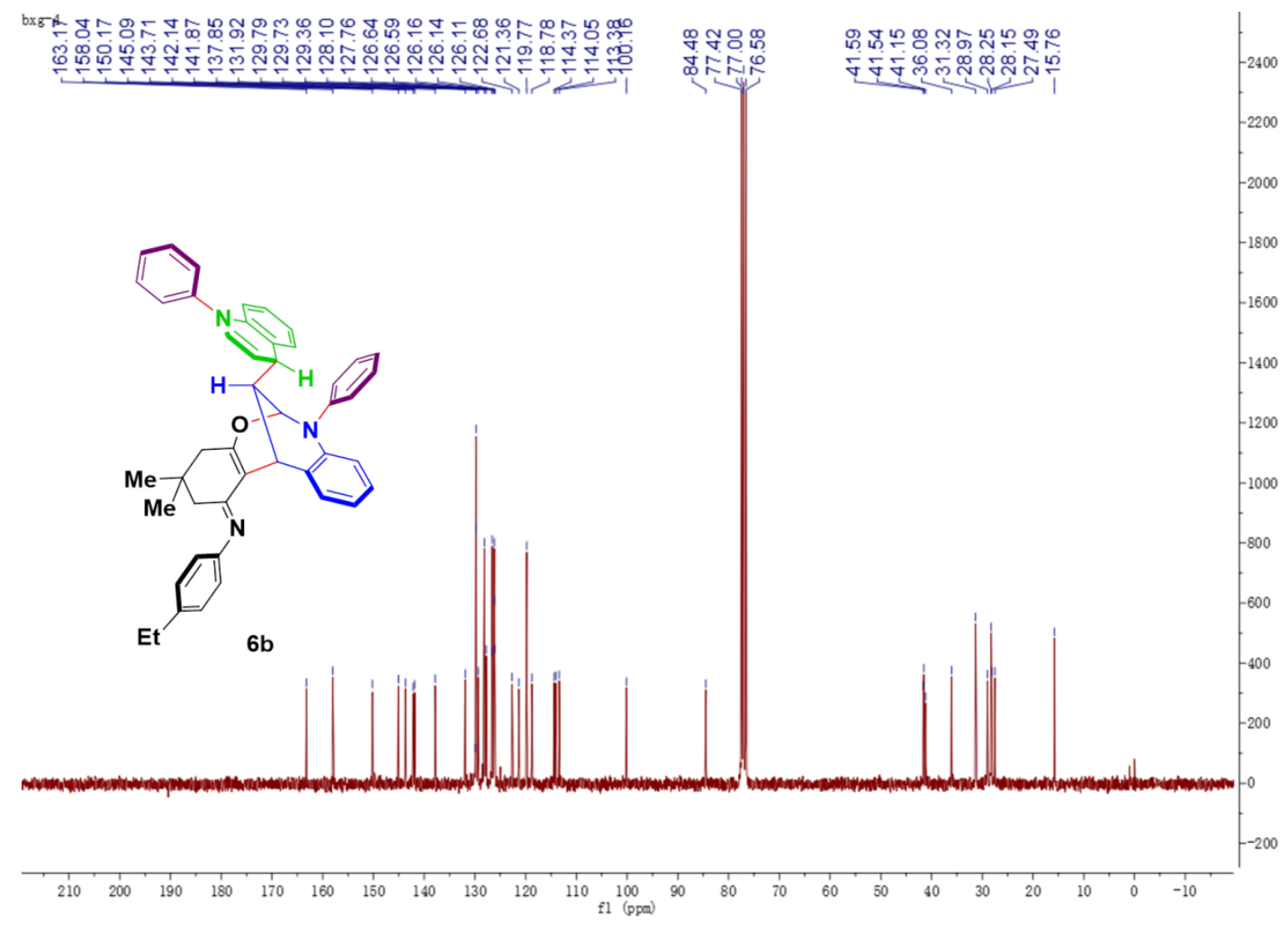

${ }^{1} \mathrm{H}$ NMR spectrum of $\mathbf{6 c}\left(400 \mathrm{MHz}, \mathrm{CDCl}_{3}\right)$

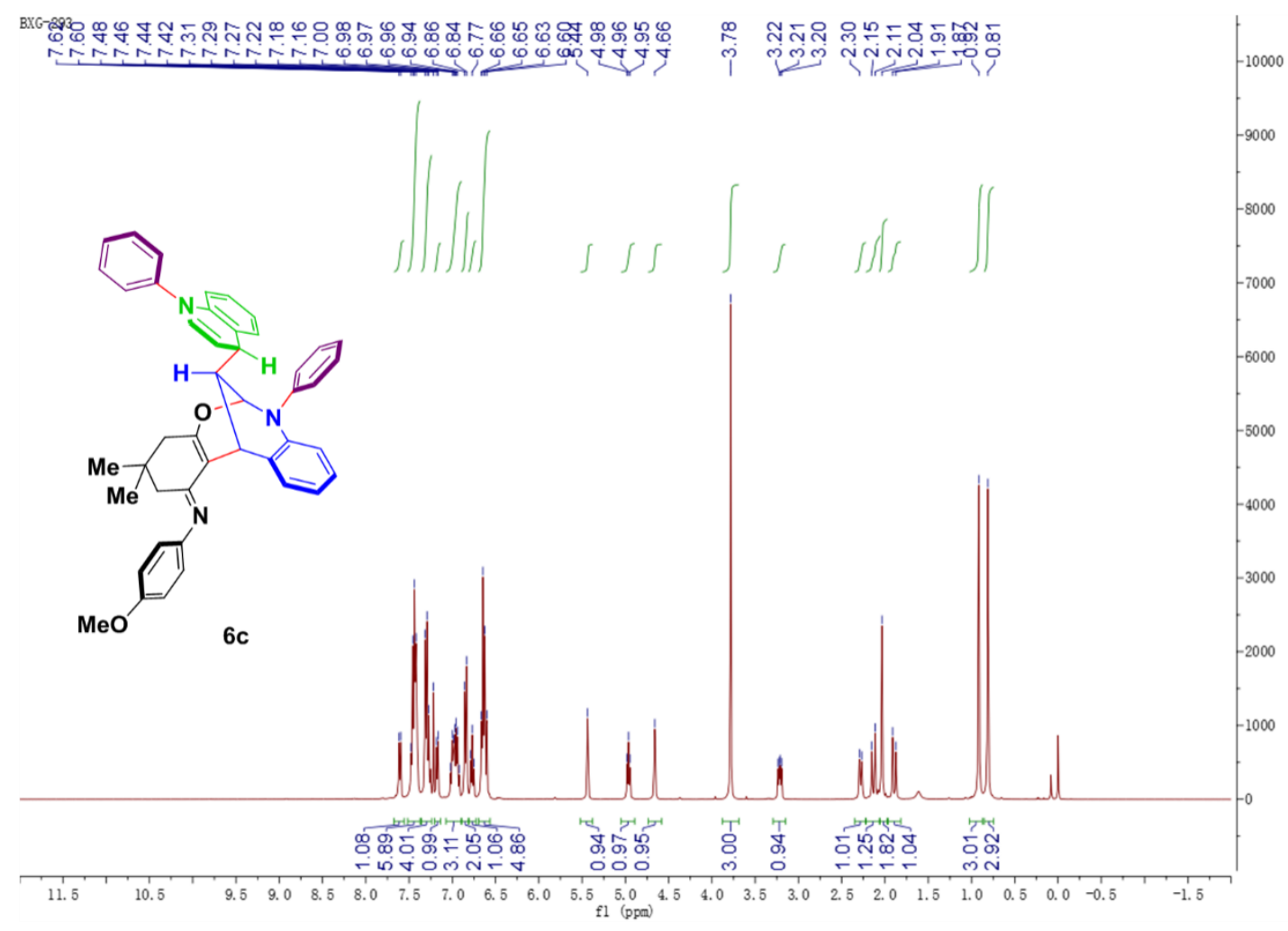

${ }^{13} \mathrm{C}$ NMR spectrum of $\mathbf{6 c}\left(100 \mathrm{MHz}, \mathrm{CDCl}_{3}\right)$ 


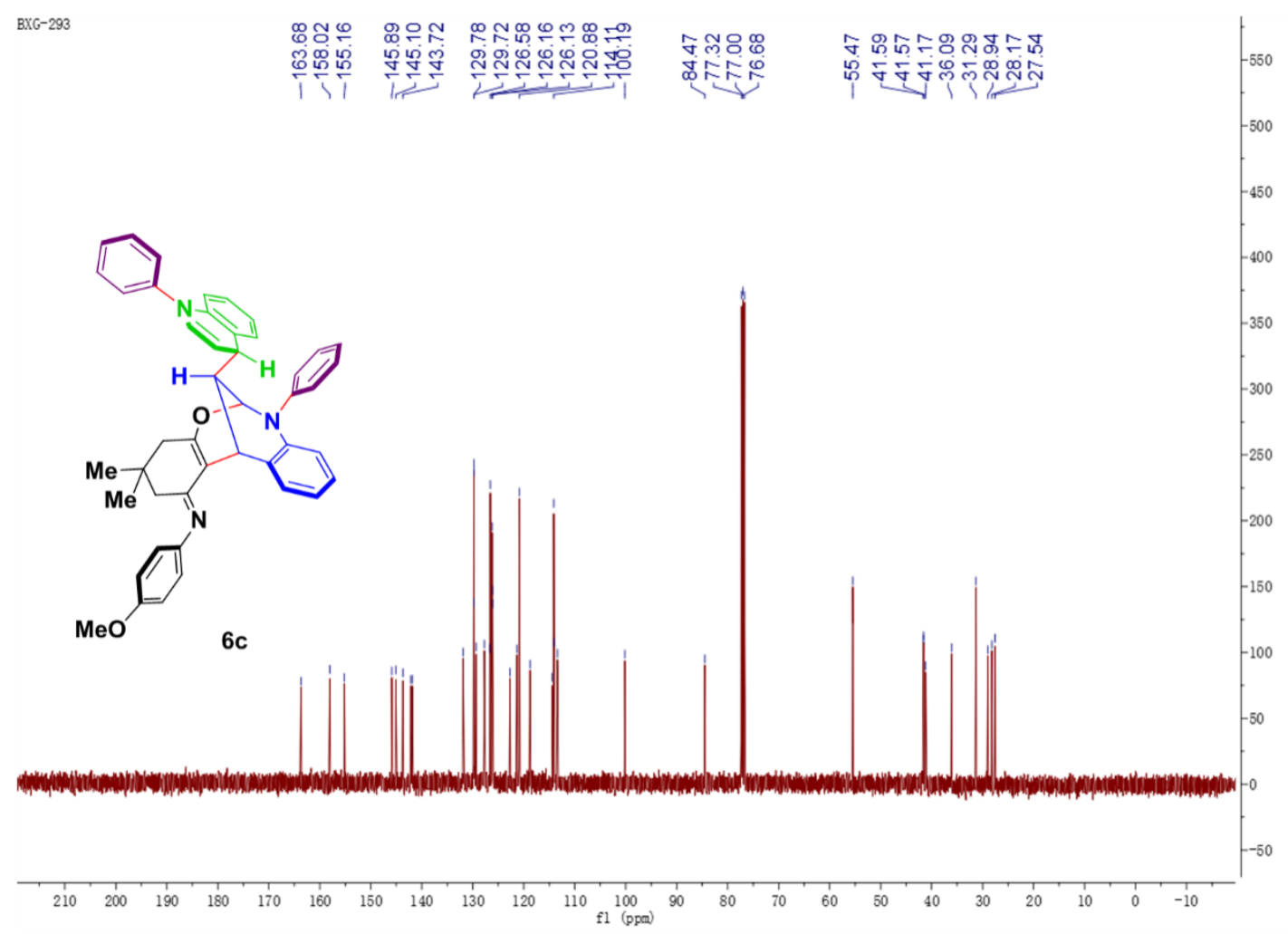

${ }^{1} \mathrm{H}$ NMR spectrum of $\mathbf{6 d}\left(400 \mathrm{MHz}, \mathrm{CDCl}_{3}\right)$

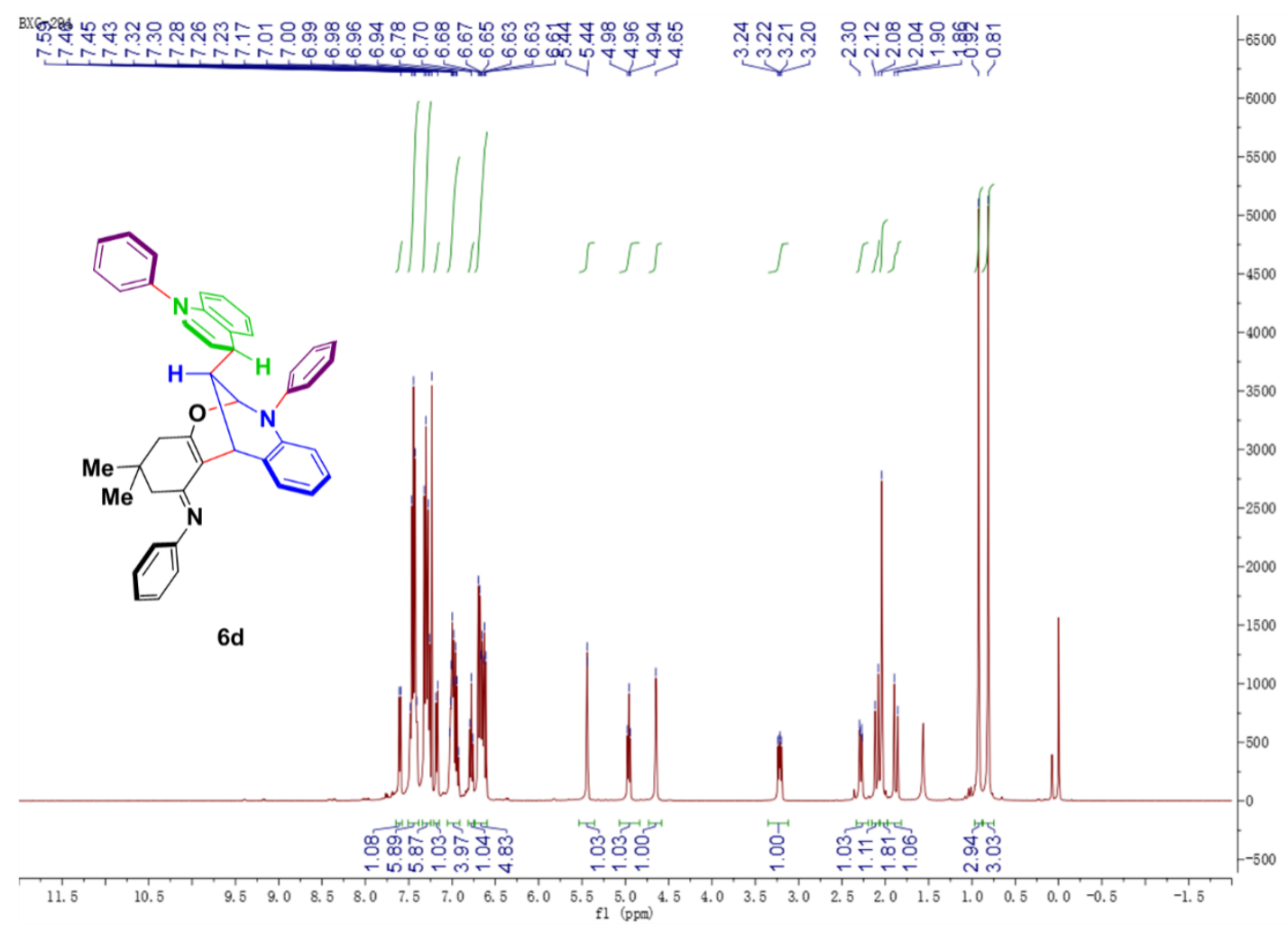

${ }^{13} \mathrm{C}$ NMR spectrum of $\mathbf{6 d}\left(100 \mathrm{MHz}, \mathrm{CDCl}_{3}\right)$ 


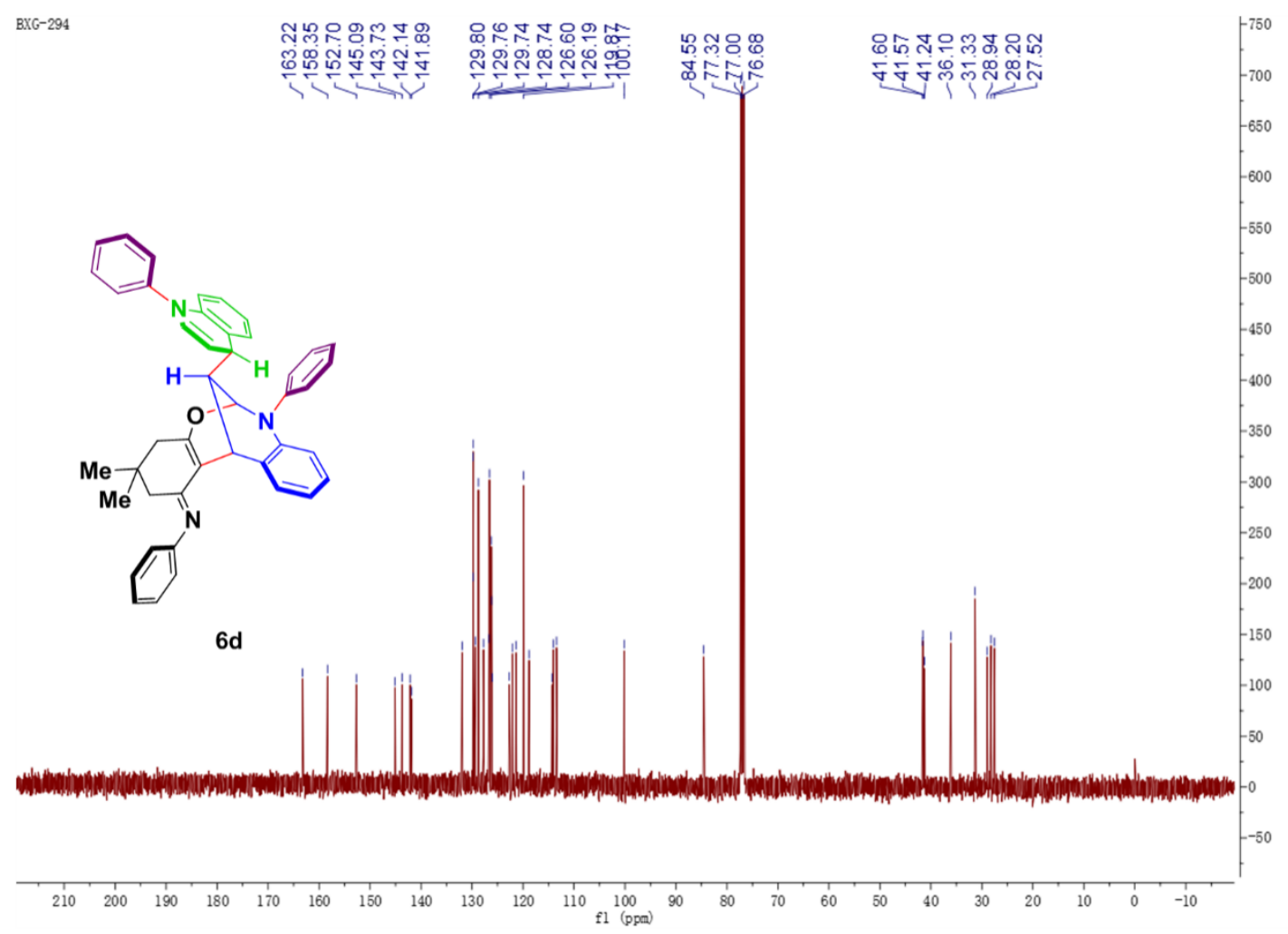

${ }^{1} \mathrm{H}$ NMR spectrum of $\mathbf{6 e}\left(400 \mathrm{MHz}, \mathrm{CDCl}_{3}\right)$

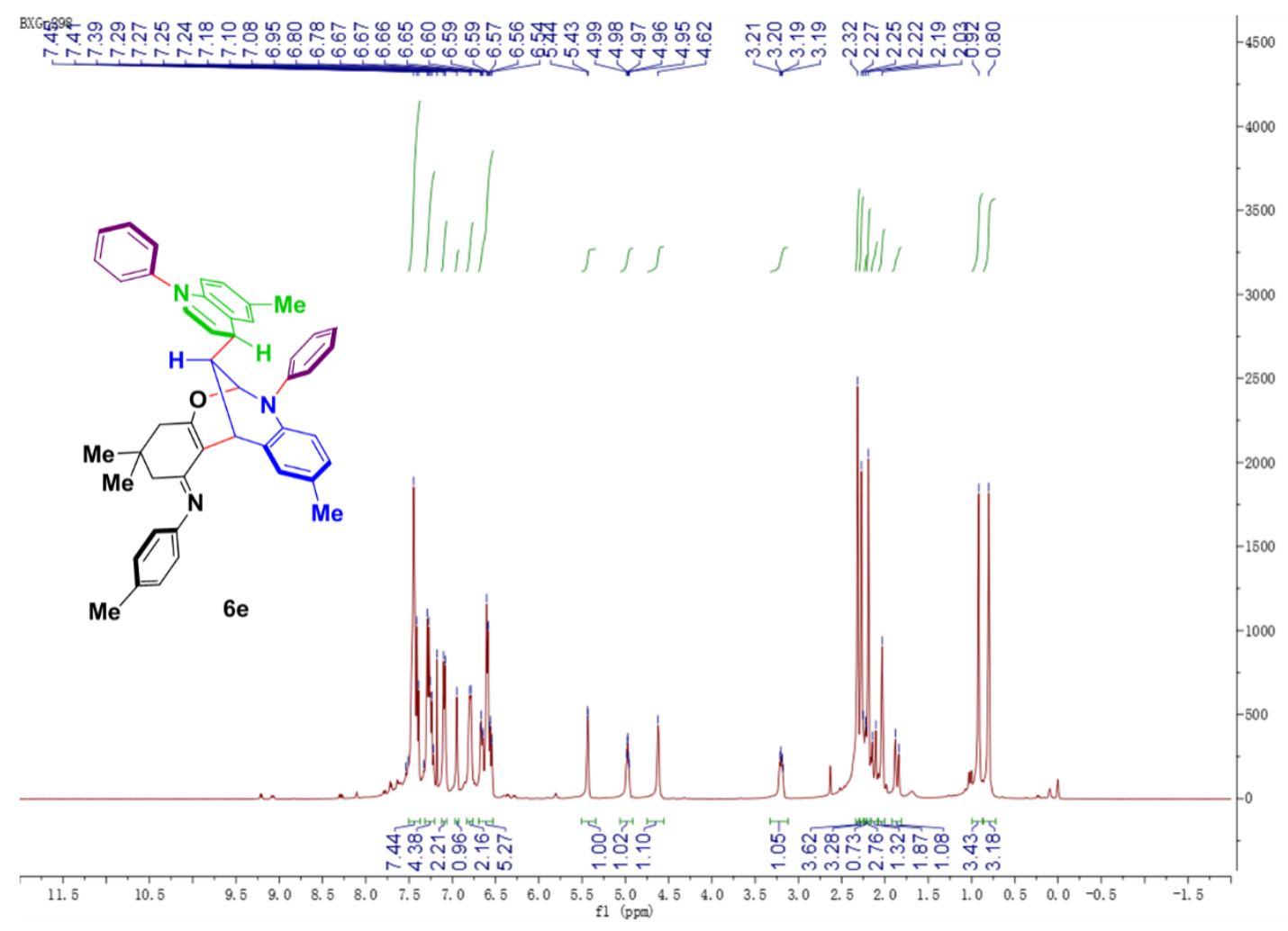

${ }^{13} \mathrm{C}$ NMR spectrum of $\mathbf{6 e}\left(100 \mathrm{MHz}, \mathrm{CDCl}_{3}\right)$ 


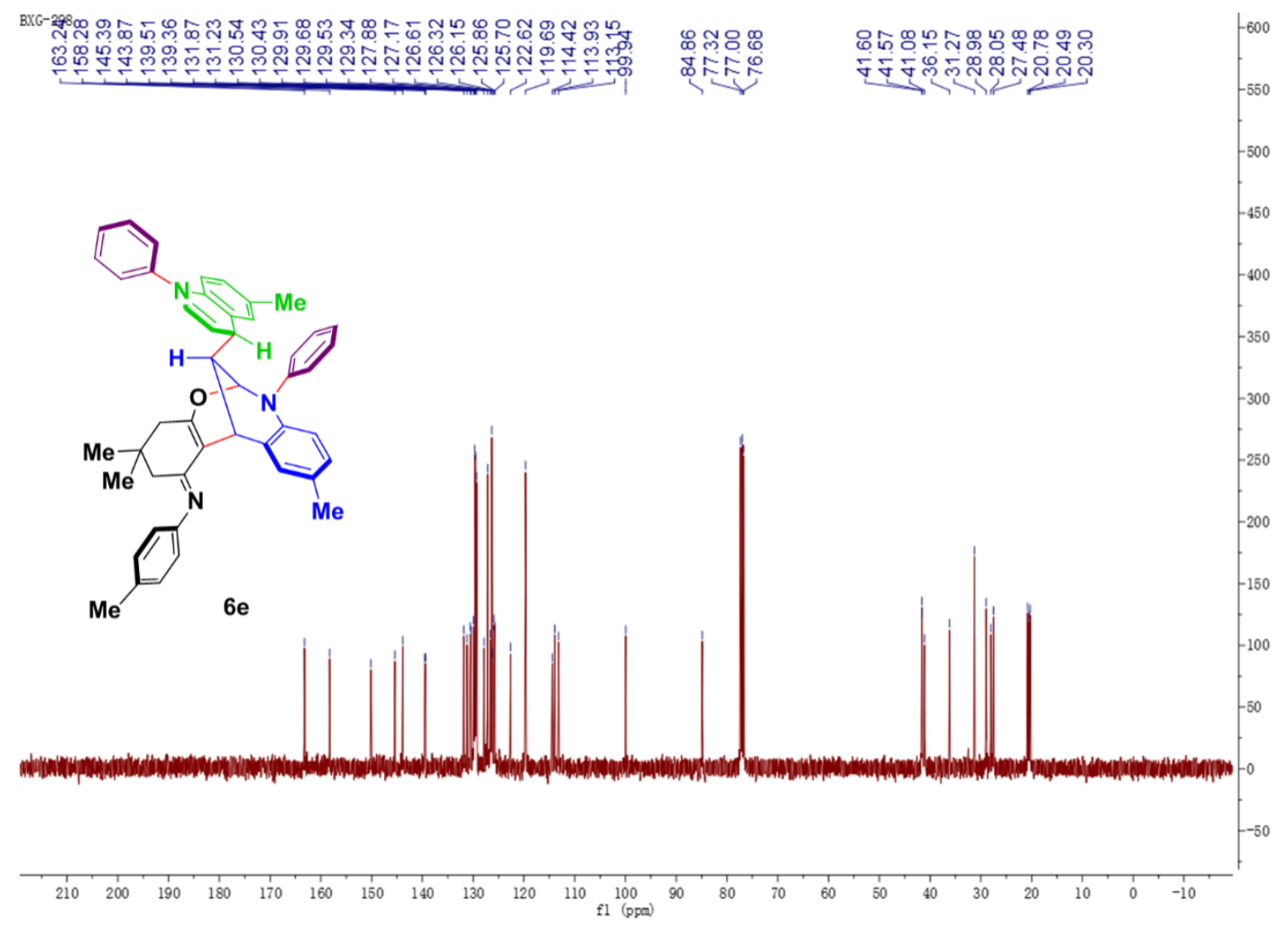

${ }^{1} \mathrm{H}$ NMR spectrum of $\mathbf{6 f}\left(400 \mathrm{MHz}, \mathrm{CDCl}_{3}\right)$

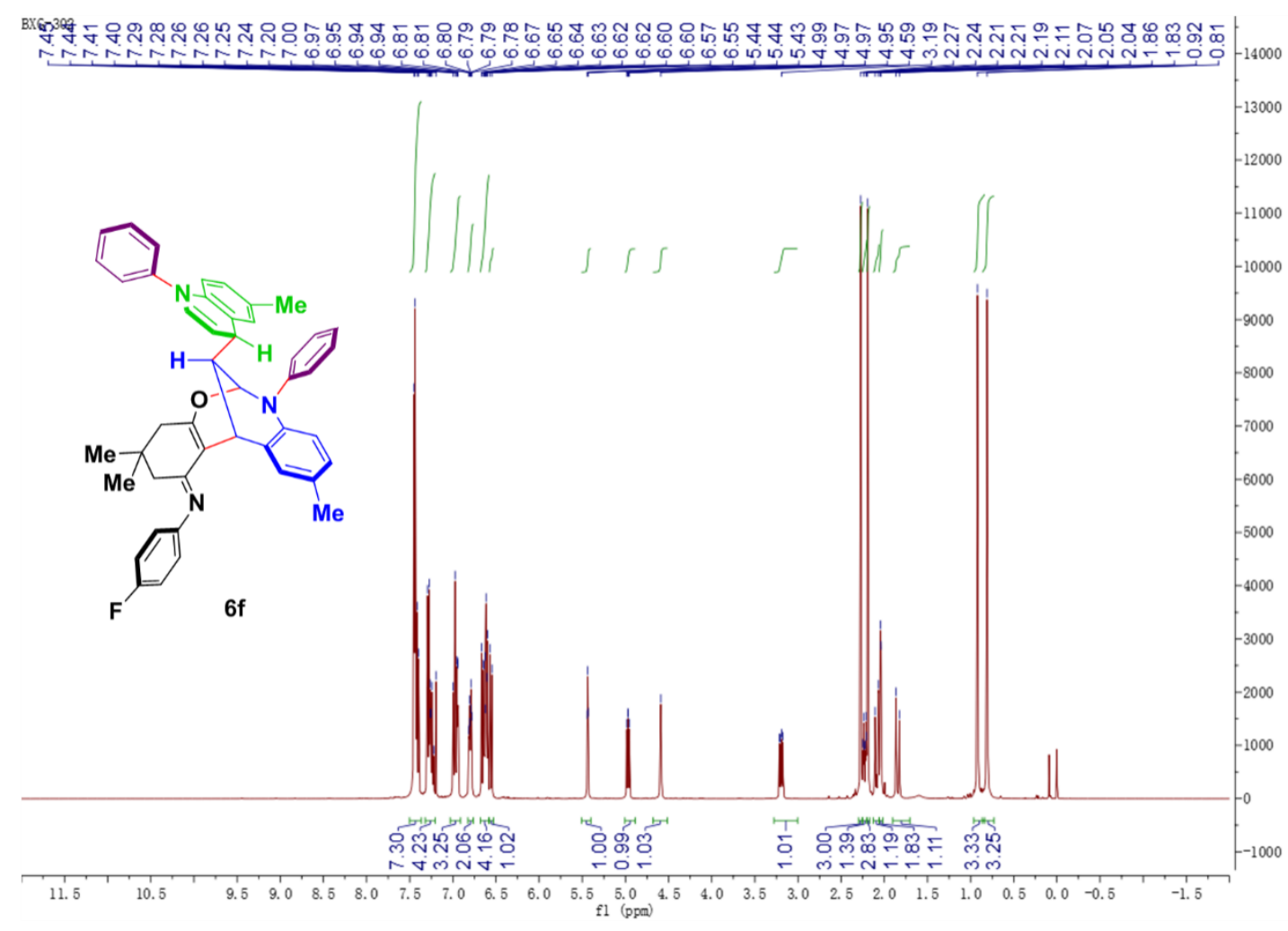

${ }^{13} \mathrm{C}$ NMR spectrum of $\mathbf{6 f}\left(100 \mathrm{MHz}, \mathrm{CDCl}_{3}\right)$ 


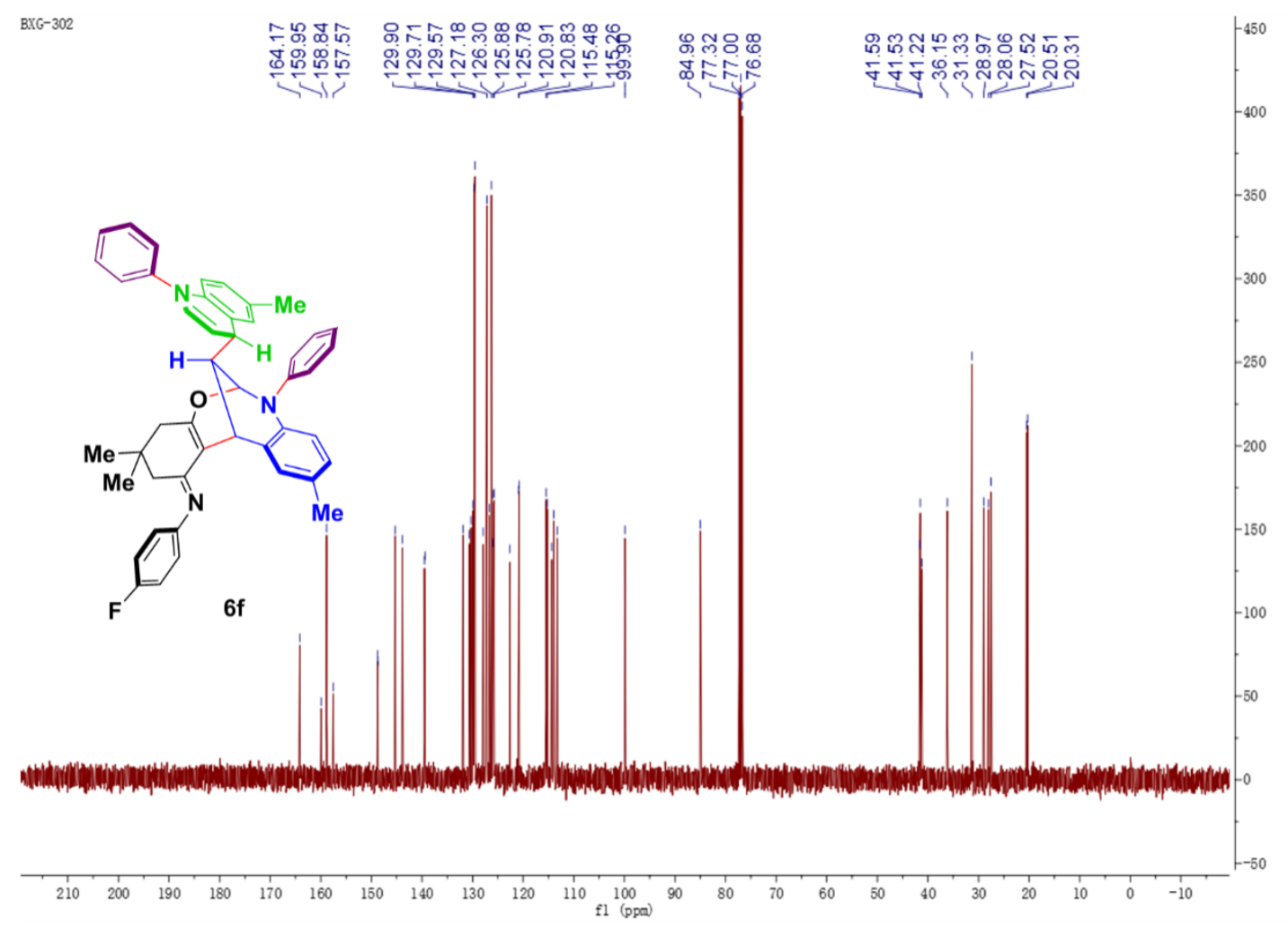

${ }^{1} \mathrm{H}$ NMR spectrum of $\mathbf{6 g}\left(300 \mathrm{MHz}, \mathrm{CDCl}_{3}\right)$

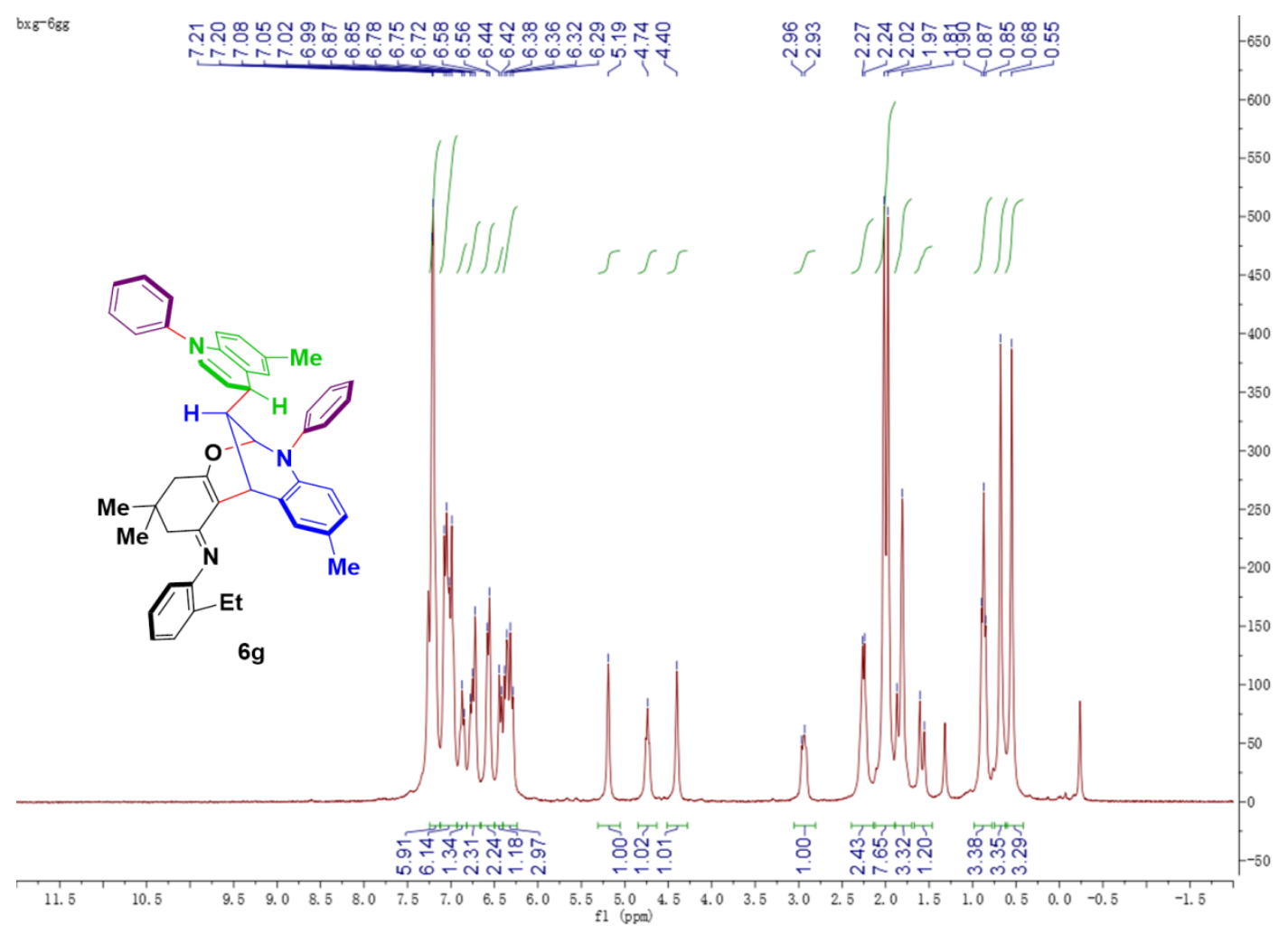

${ }^{13} \mathrm{C}$ NMR spectrum of $\mathbf{6 g}\left(75 \mathrm{MHz}, \mathrm{CDCl}_{3}\right)$ 


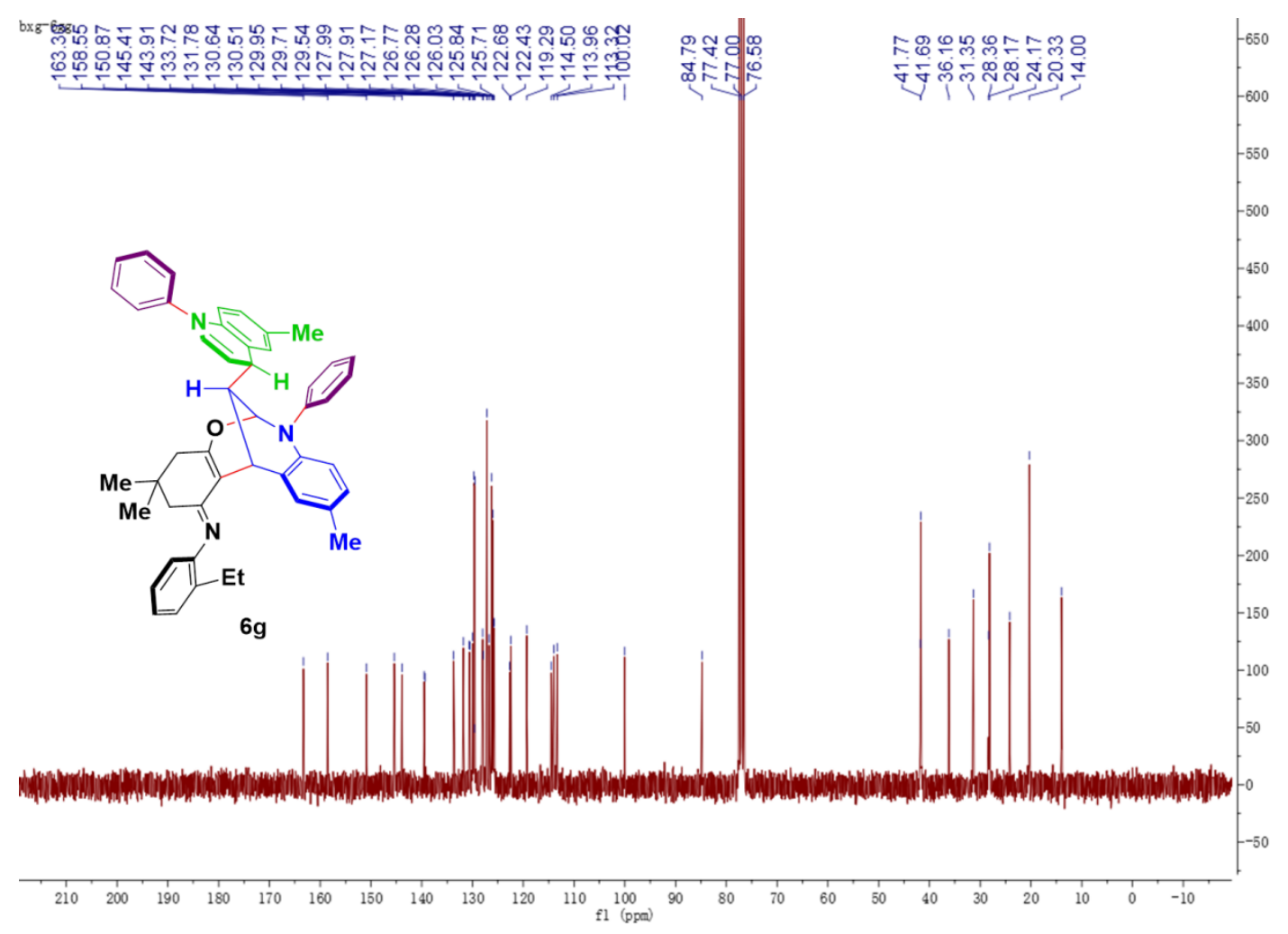

${ }^{1} \mathrm{H}$ NMR spectrum of $\mathbf{6 h}\left(300 \mathrm{MHz}, \mathrm{CDCl}_{3}\right)$

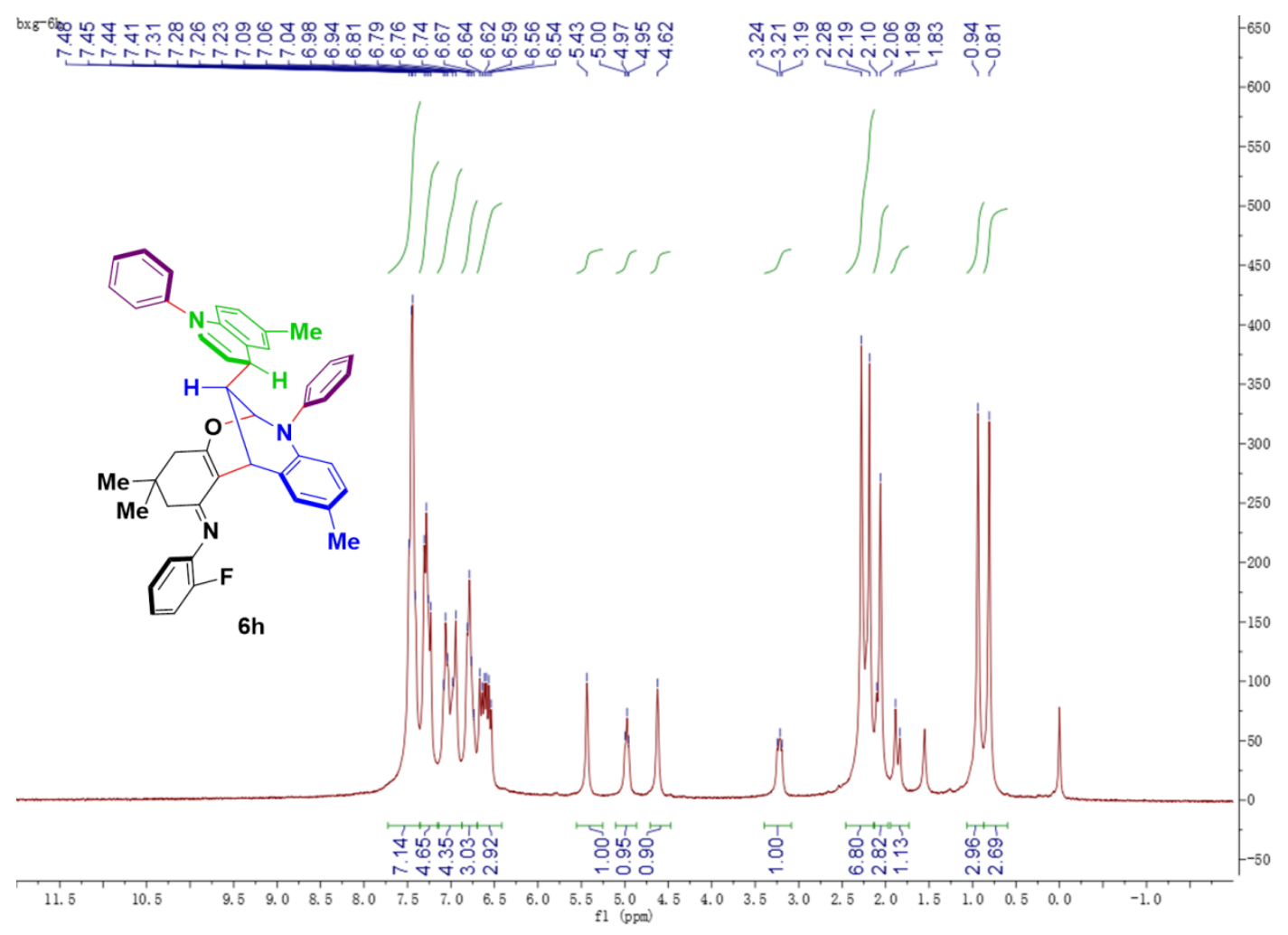

${ }^{13} \mathrm{C}$ NMR spectrum of $\mathbf{6 h}\left(75 \mathrm{MHz}, \mathrm{CDCl}_{3}\right)$ 


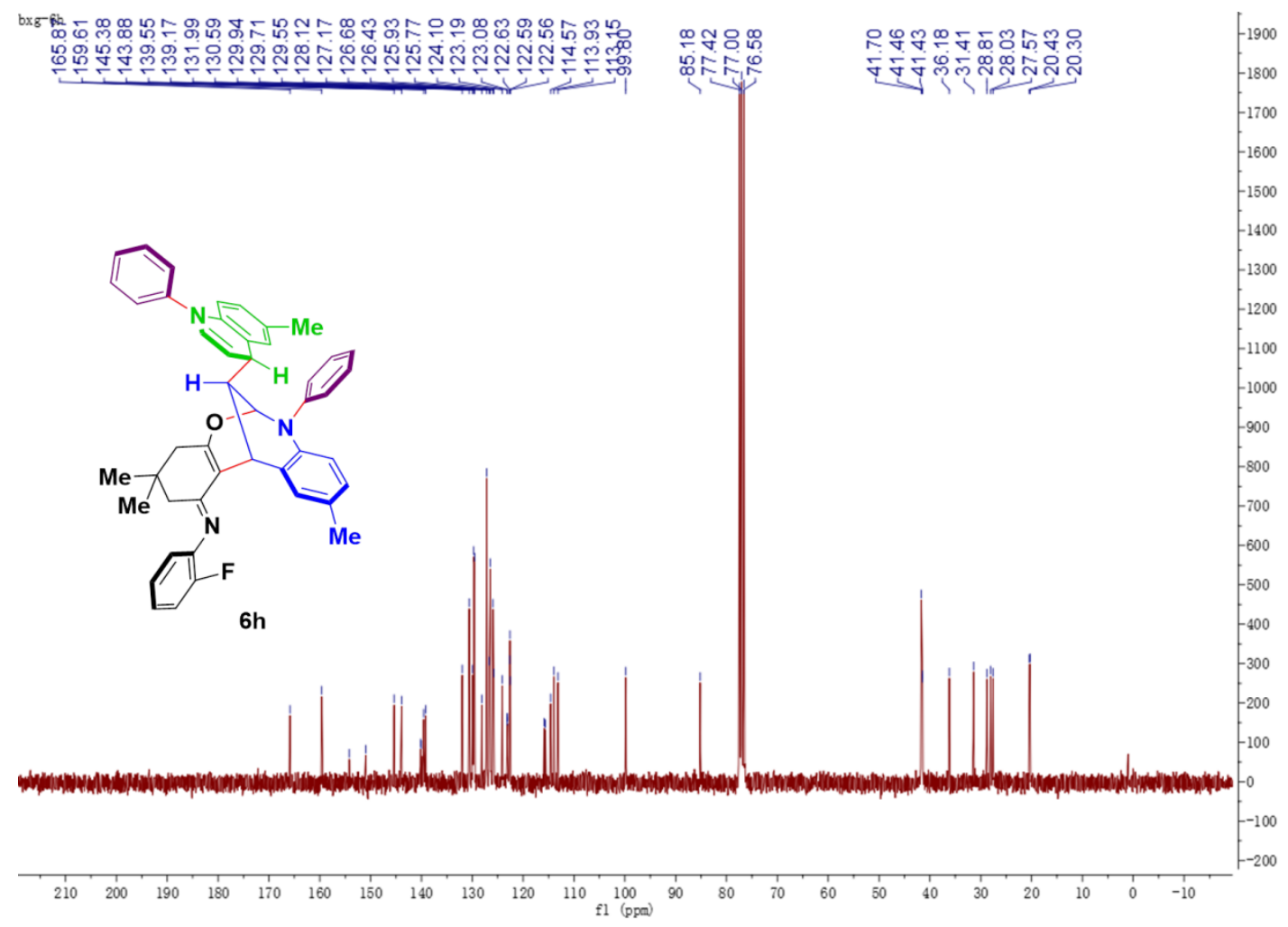

${ }^{1} \mathrm{H}$ NMR spectrum of $\mathbf{6 i}\left(400 \mathrm{MHz}, \mathrm{CDCl}_{3}\right)$

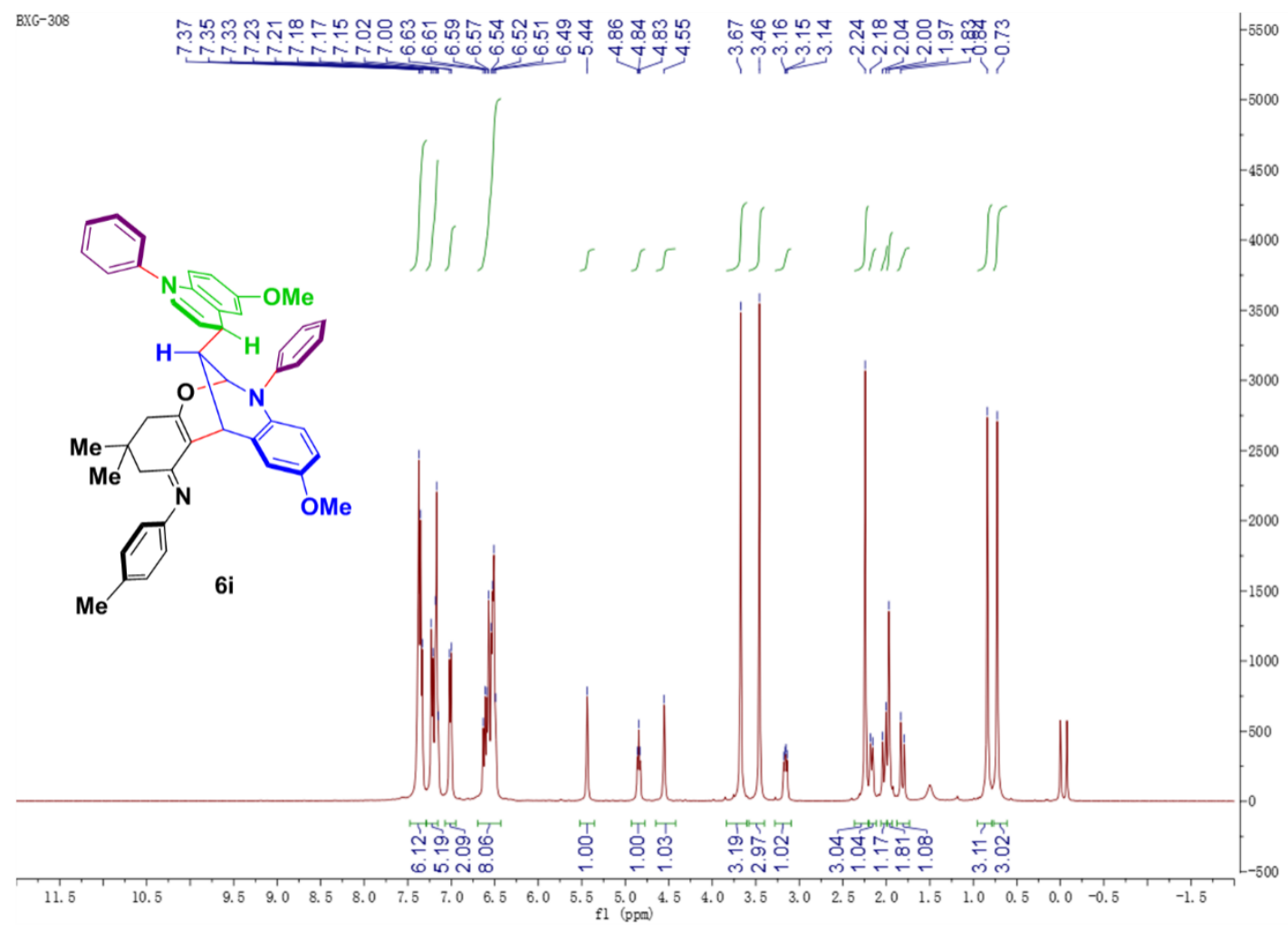

${ }^{13} \mathrm{C}$ NMR spectrum of $\mathbf{6 i}\left(100 \mathrm{MHz}, \mathrm{CDCl}_{3}\right)$ 


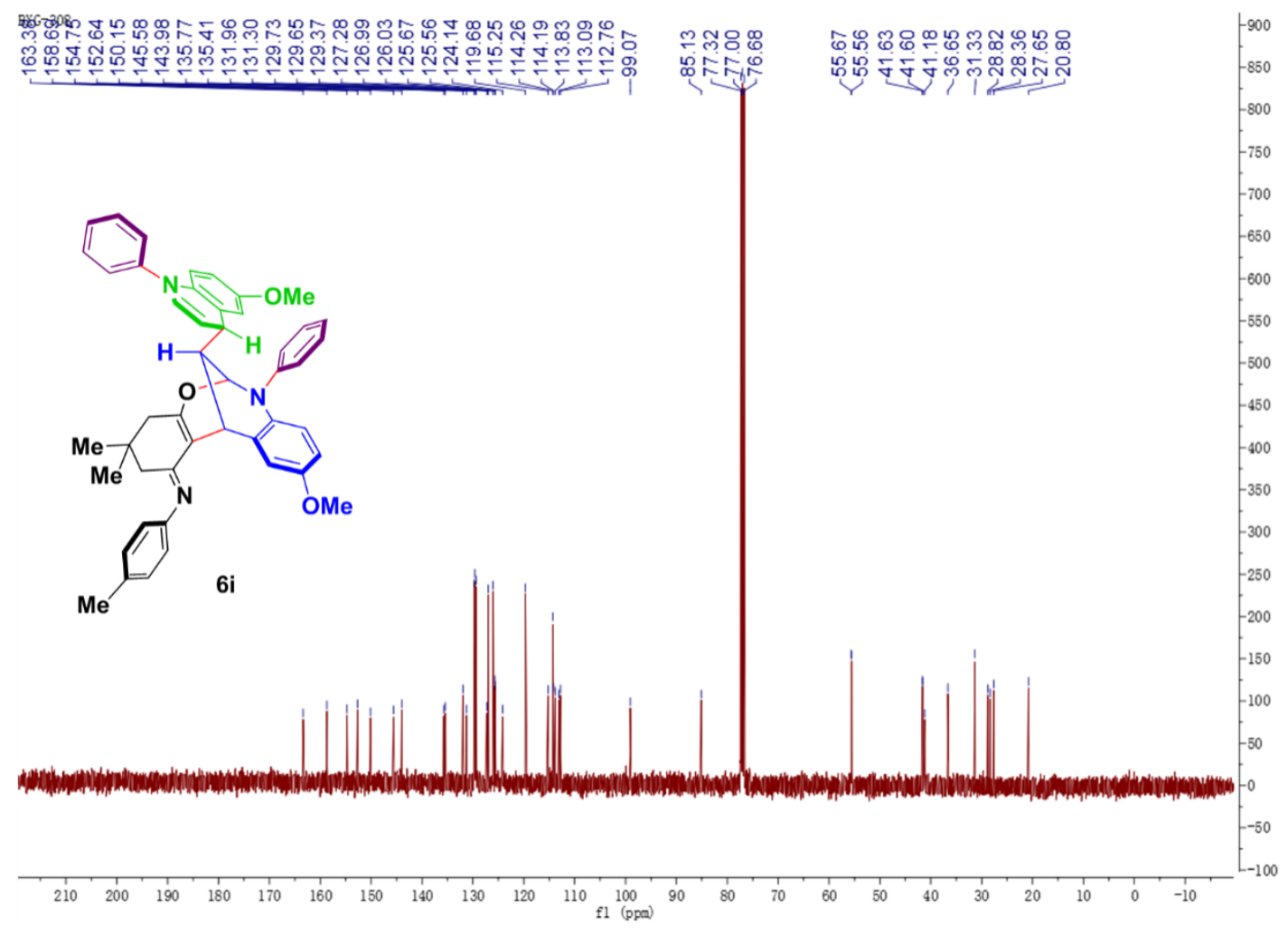

${ }^{1} \mathrm{H}$ NMR spectrum of $\mathbf{6 j}\left(400 \mathrm{MHz}, \mathrm{CDCl}_{3}\right)$

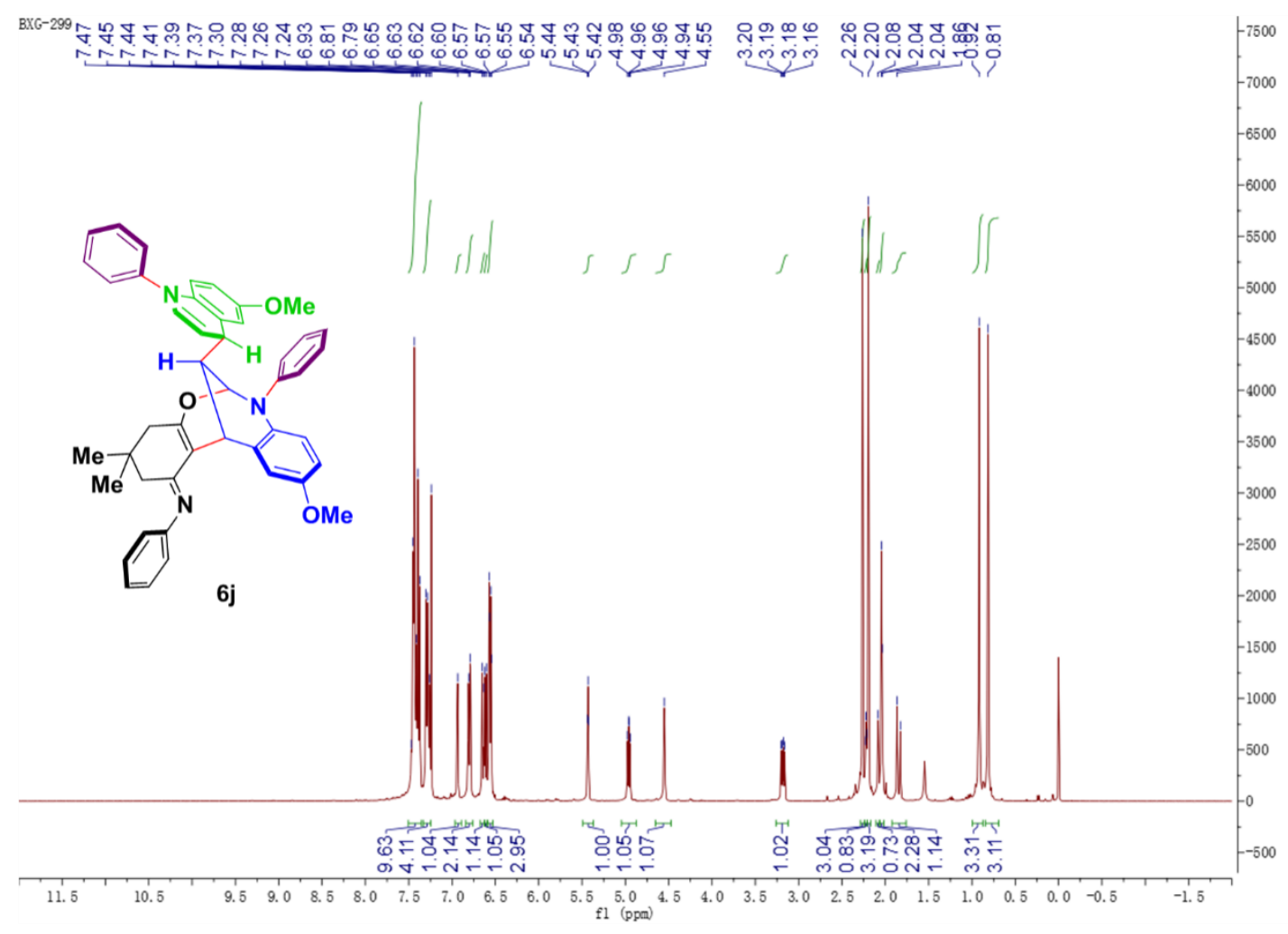

${ }^{13} \mathrm{C}$ NMR spectrum of $\mathbf{6 j}\left(100 \mathrm{MHz}, \mathrm{CDCl}_{3}\right)$ 


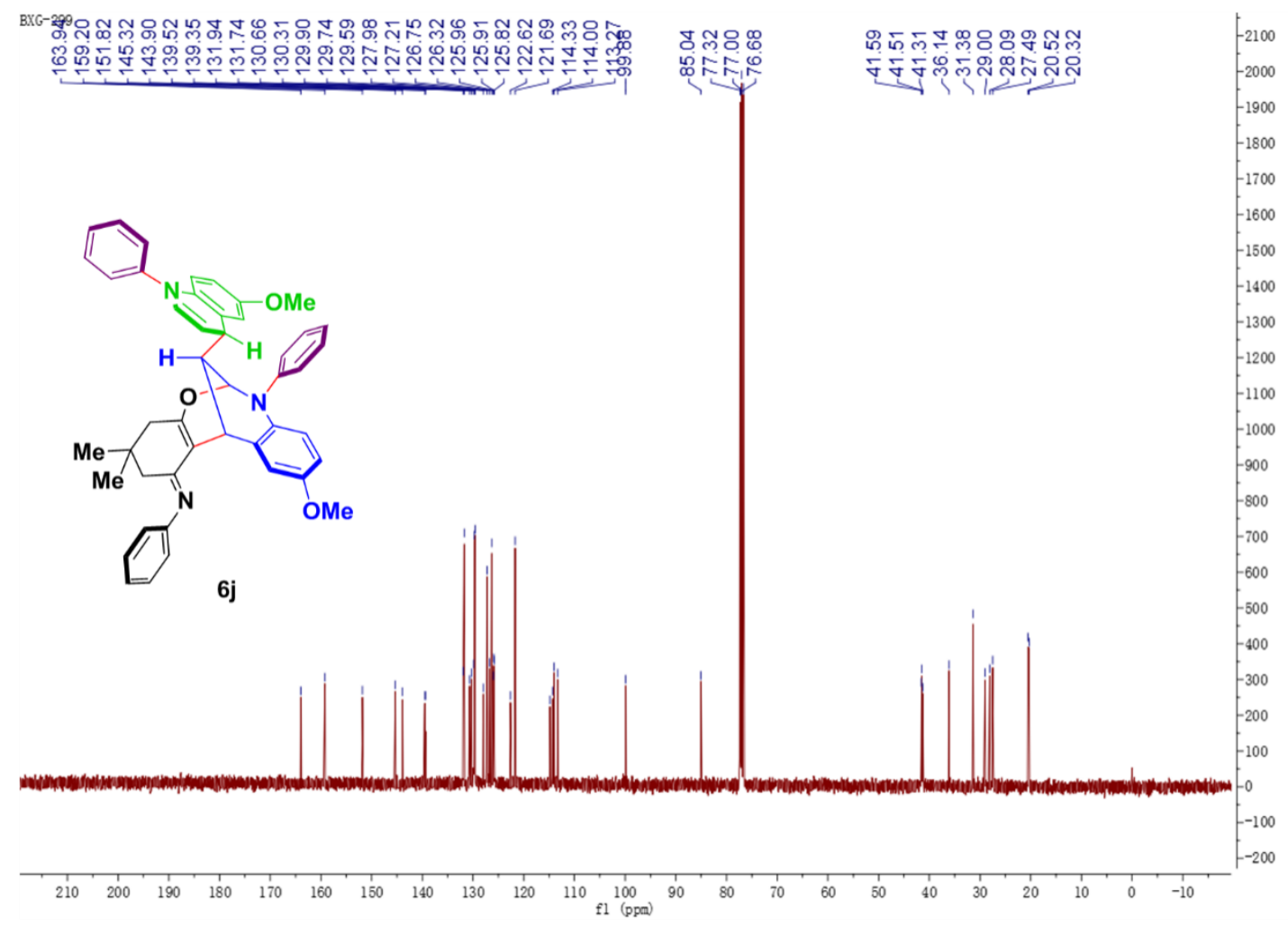

${ }^{1} \mathrm{H}$ NMR spectrum of $\mathbf{6 k}\left(400 \mathrm{MHz}, \mathrm{CDCl}_{3}\right)$

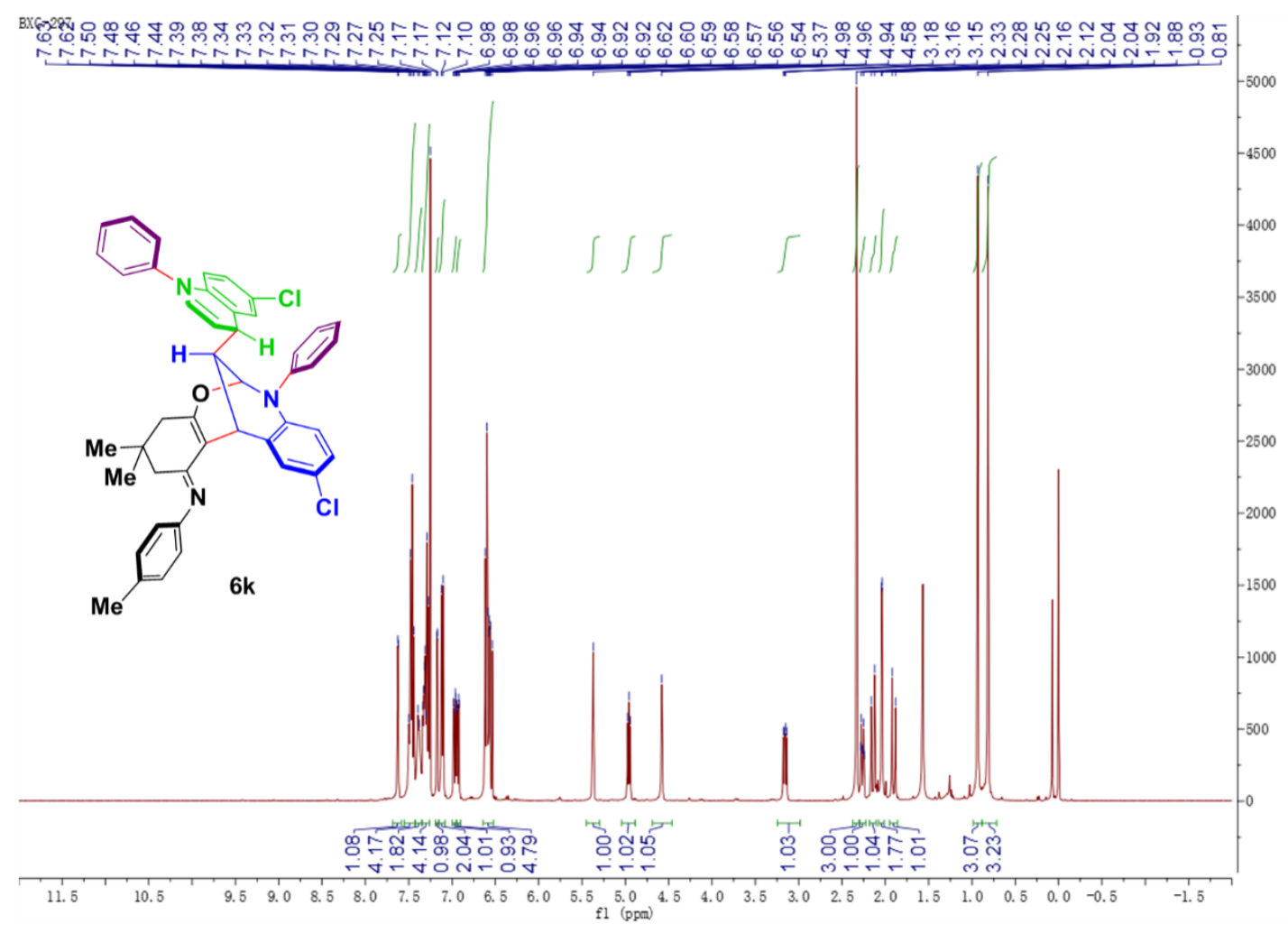

${ }^{13} \mathrm{C}$ NMR spectrum of $\mathbf{6 k}\left(100 \mathrm{MHz}, \mathrm{CDCl}_{3}\right)$ 


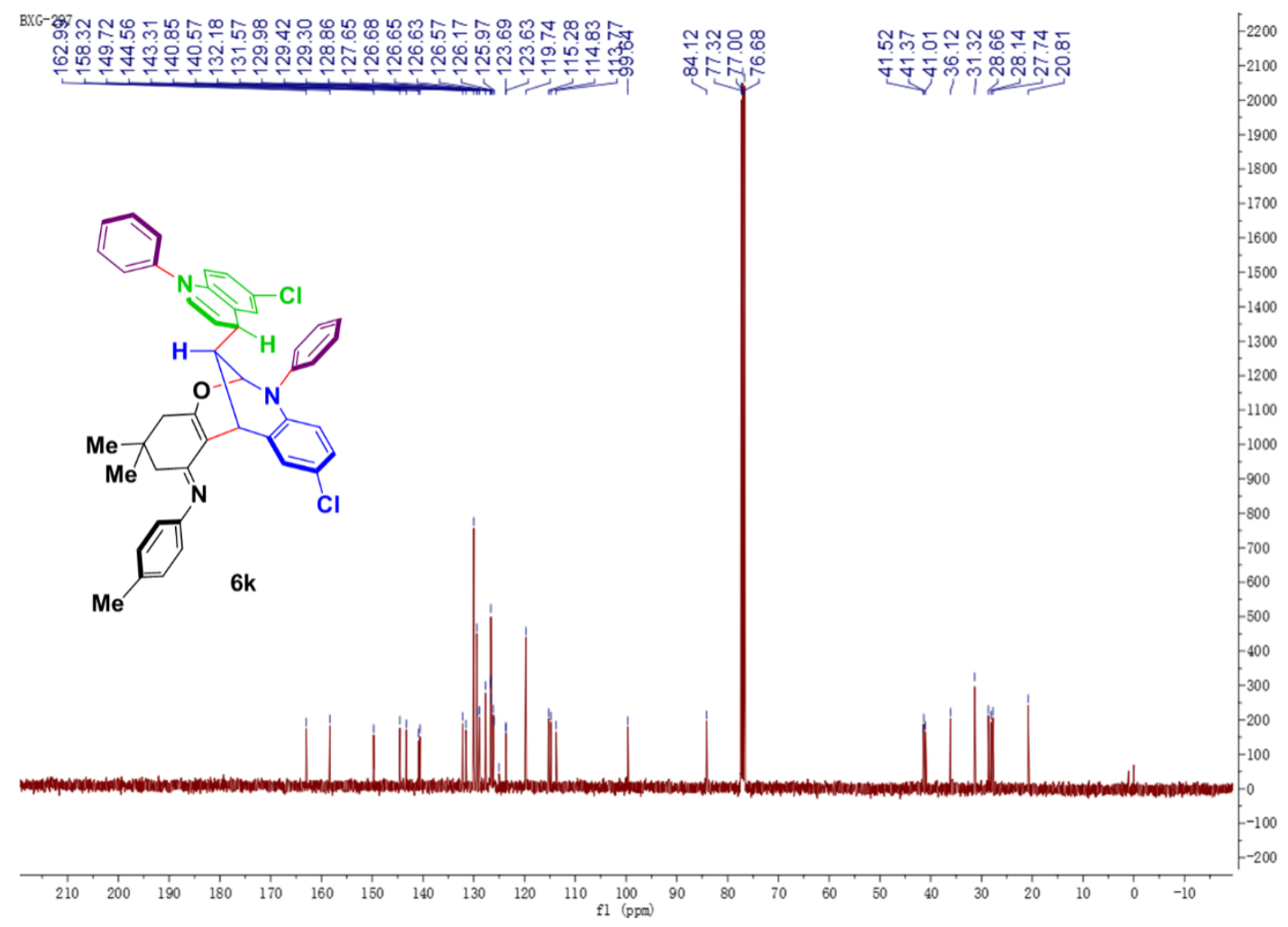

${ }^{1} \mathrm{H}$ NMR spectrum of $6 \mathbf{l}\left(400 \mathrm{MHz}, \mathrm{CDCl}_{3}\right)$

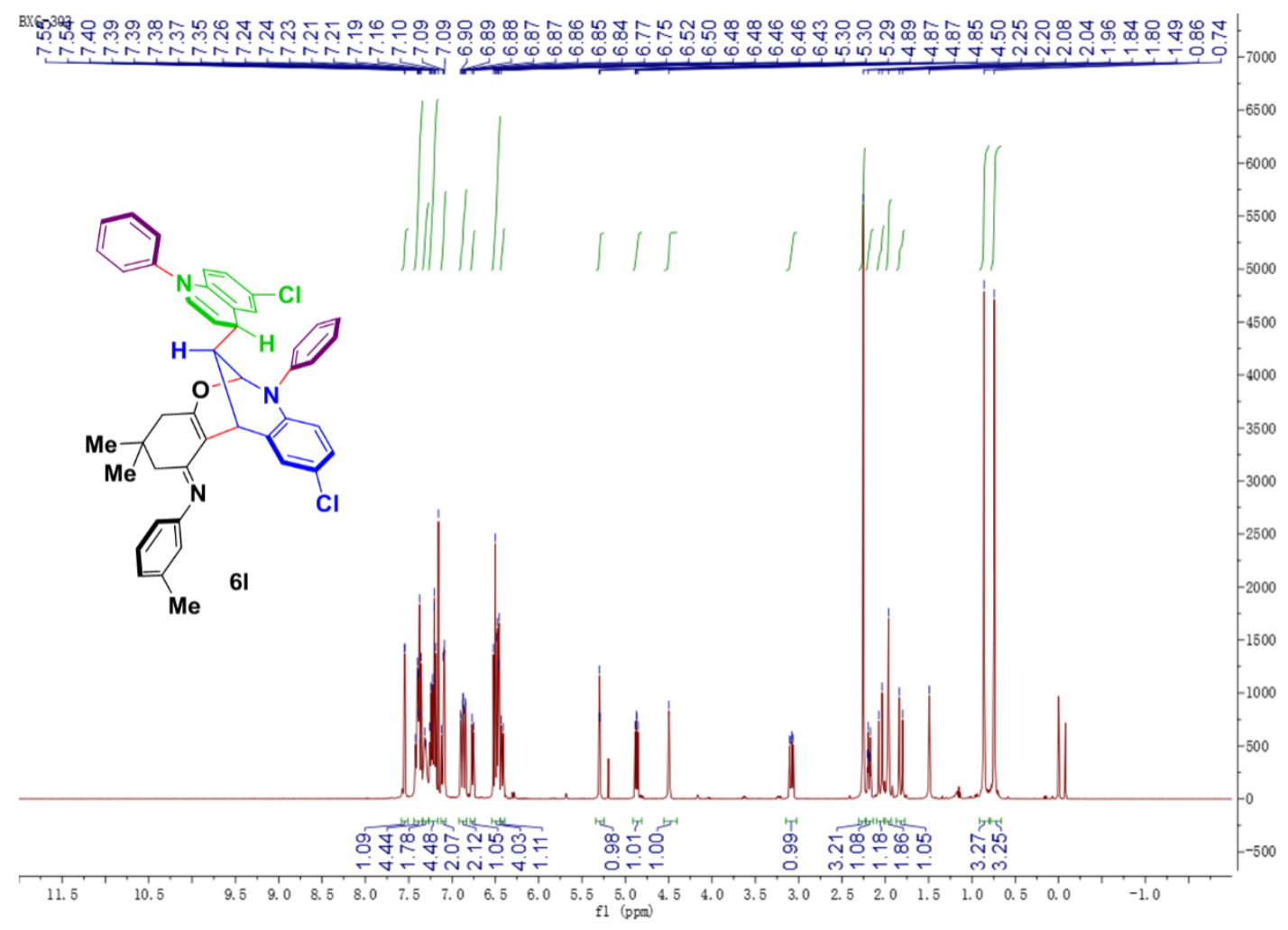

${ }^{13} \mathrm{C}$ NMR spectrum of $6 \mathbf{l}\left(100 \mathrm{MHz}, \mathrm{CDCl}_{3}\right)$ 


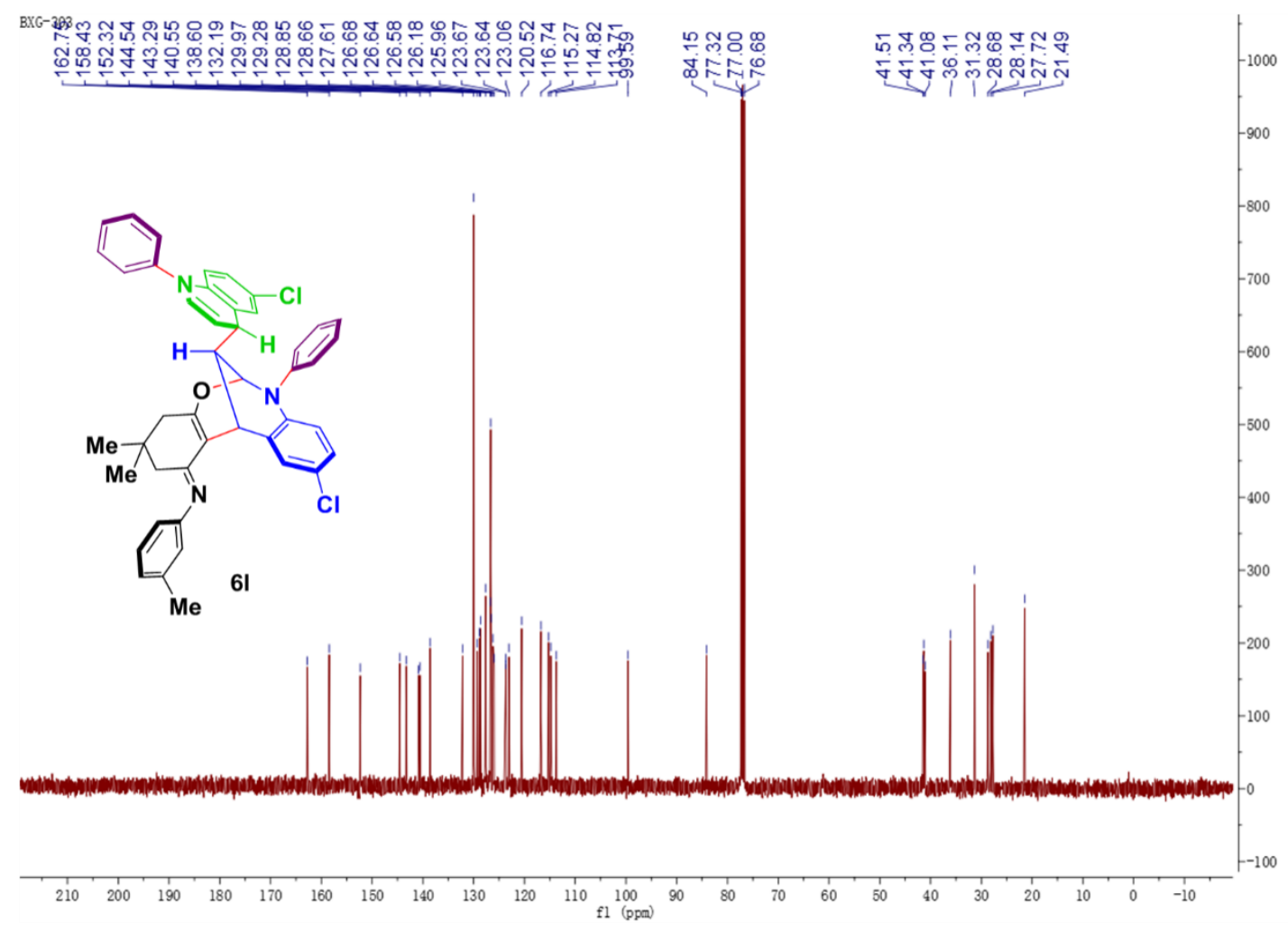

${ }^{1} \mathrm{H}$ NMR spectrum of $\mathbf{6 m}\left(400 \mathrm{MHz}, \mathrm{CDCl}_{3}\right)$

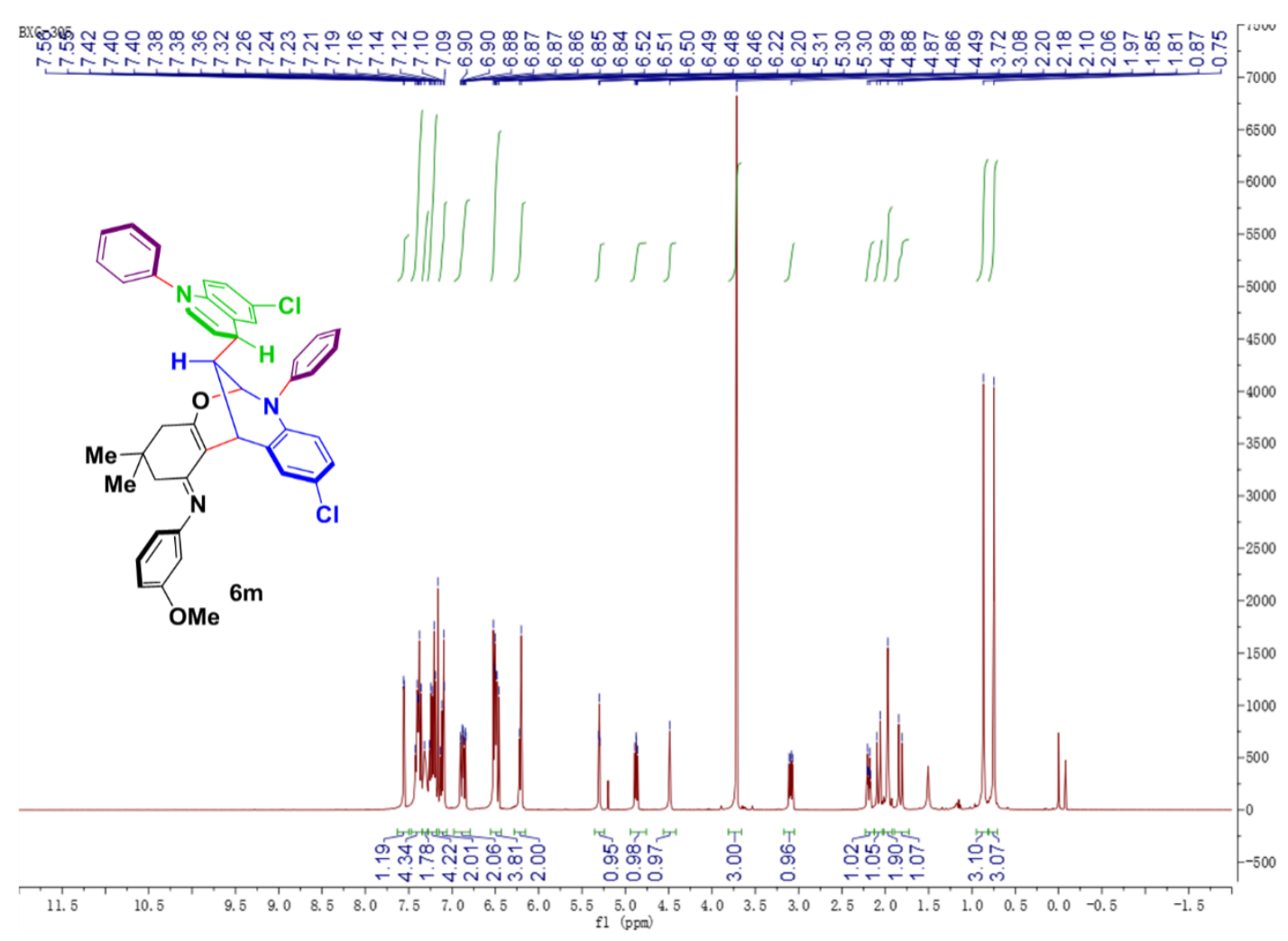

${ }^{13} \mathrm{C}$ NMR spectrum of $\mathbf{6 m}\left(100 \mathrm{MHz}, \mathrm{CDCl}_{3}\right)$ 


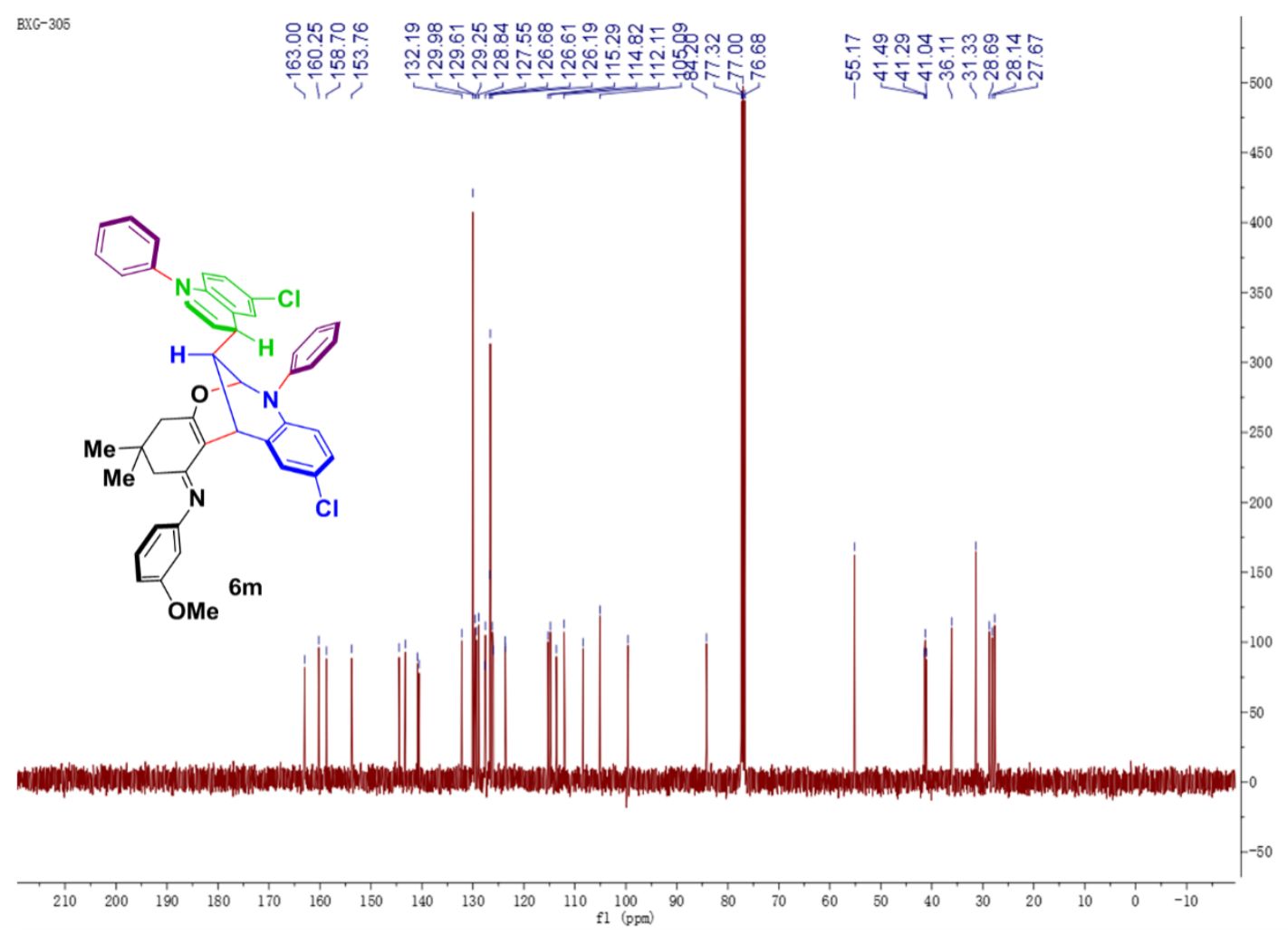

${ }^{1} \mathrm{H}$ NMR spectrum of $\mathbf{6 n}\left(400 \mathrm{MHz}, \mathrm{CDCl}_{3}\right)$

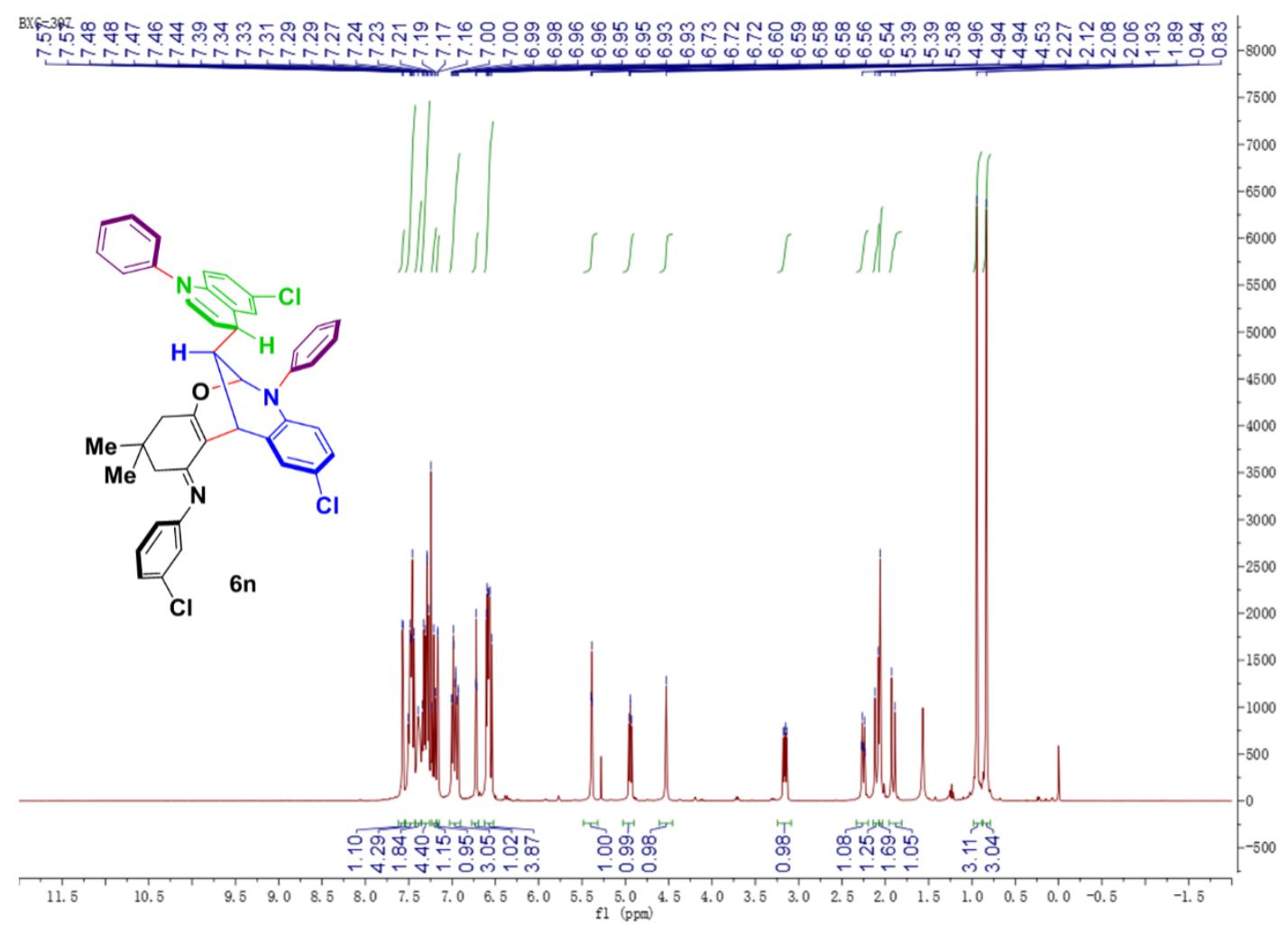

${ }^{13} \mathrm{C}$ NMR spectrum of $\mathbf{6 n}\left(100 \mathrm{MHz}, \mathrm{CDCl}_{3}\right)$ 


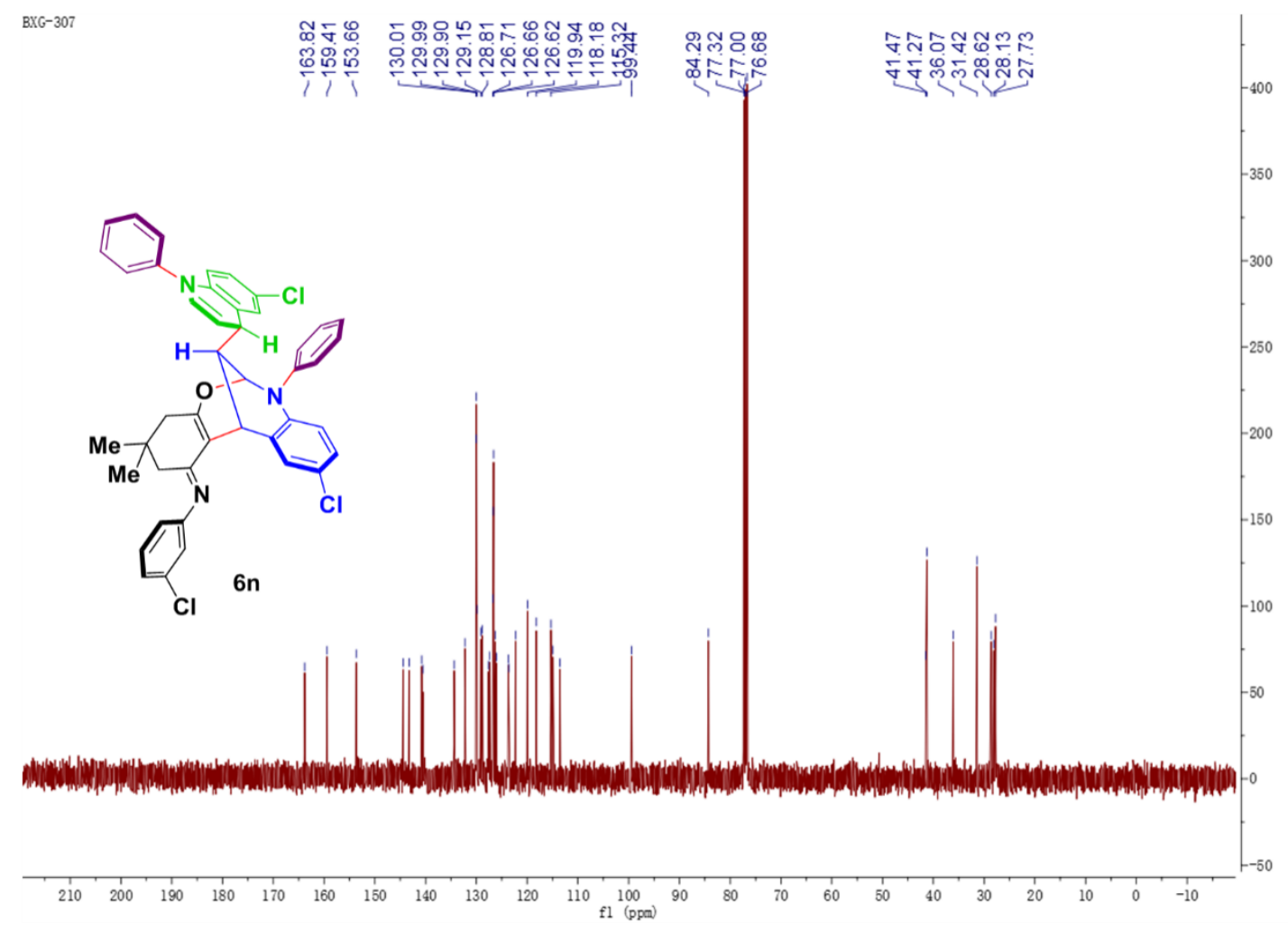

${ }^{1} \mathrm{H}$ NMR spectrum of $\mathbf{6 o}\left(400 \mathrm{MHz}, \mathrm{CDCl}_{3}\right)$

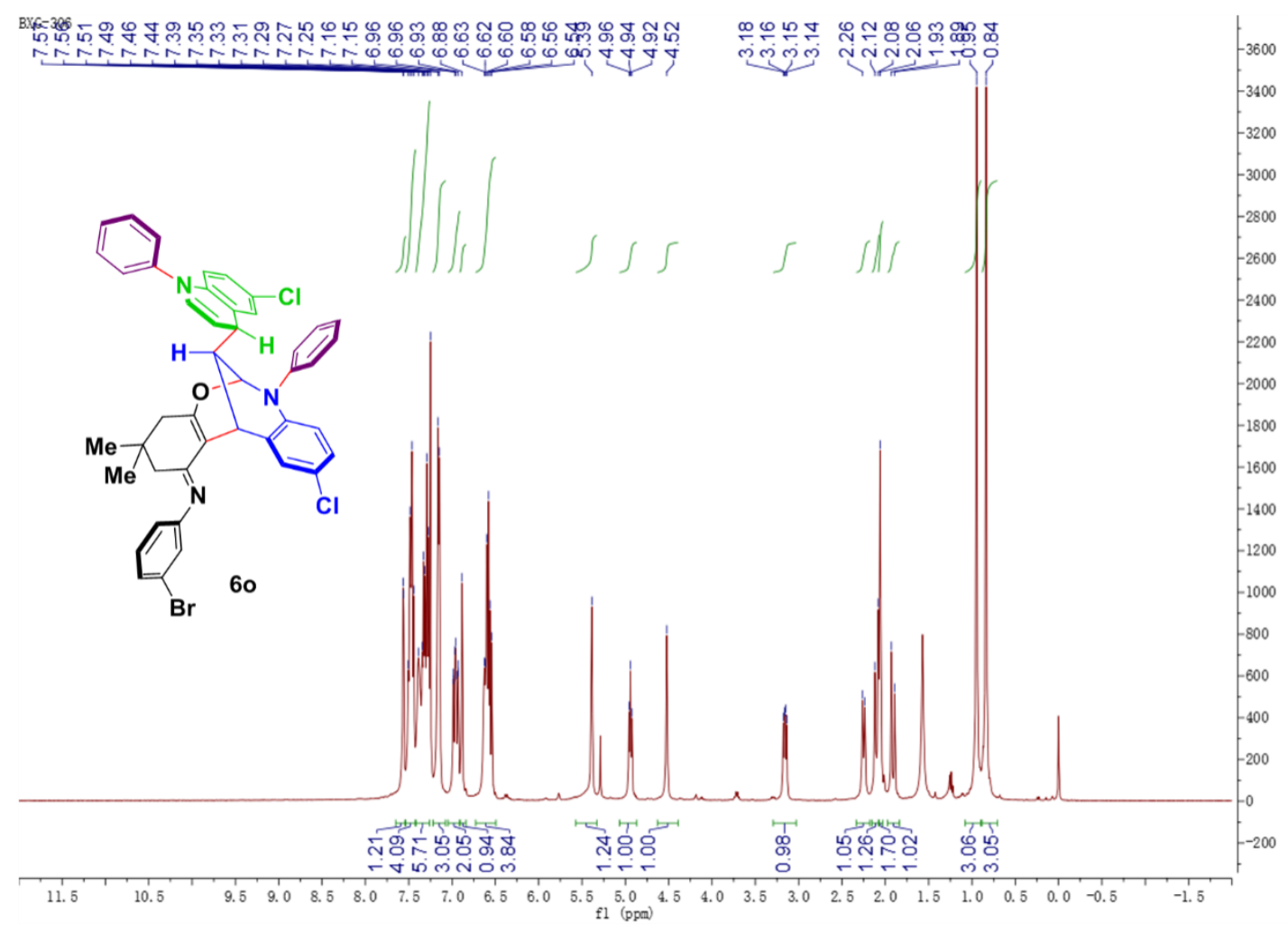

${ }^{13} \mathrm{C}$ NMR spectrum of $\mathbf{6 o}\left(100 \mathrm{MHz}, \mathrm{CDCl}_{3}\right)$ 


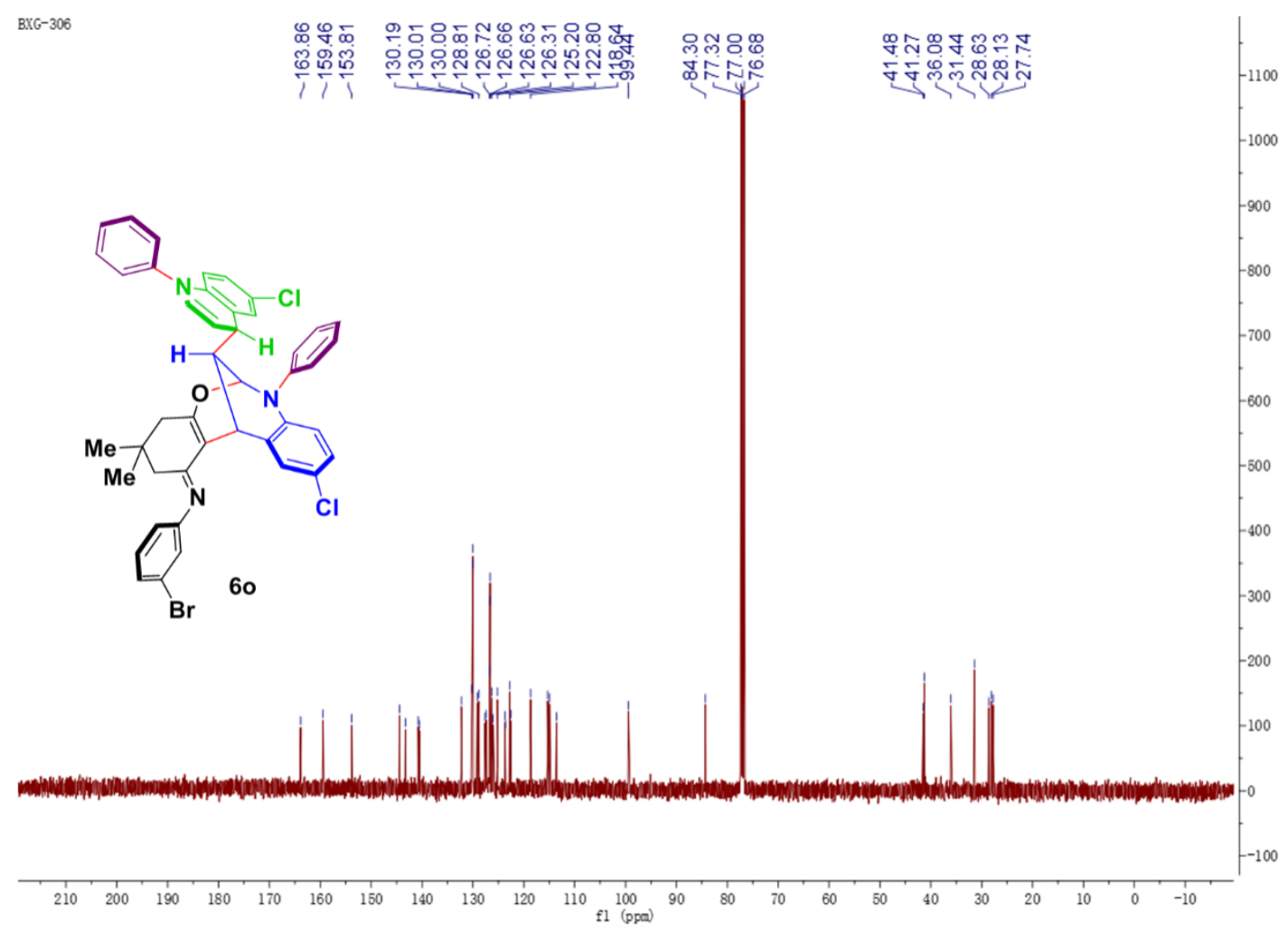

${ }^{1} \mathrm{H}$ NMR spectrum of $7 \mathbf{a}\left(400 \mathrm{MHz}, \mathrm{CDCl}_{3}\right)$

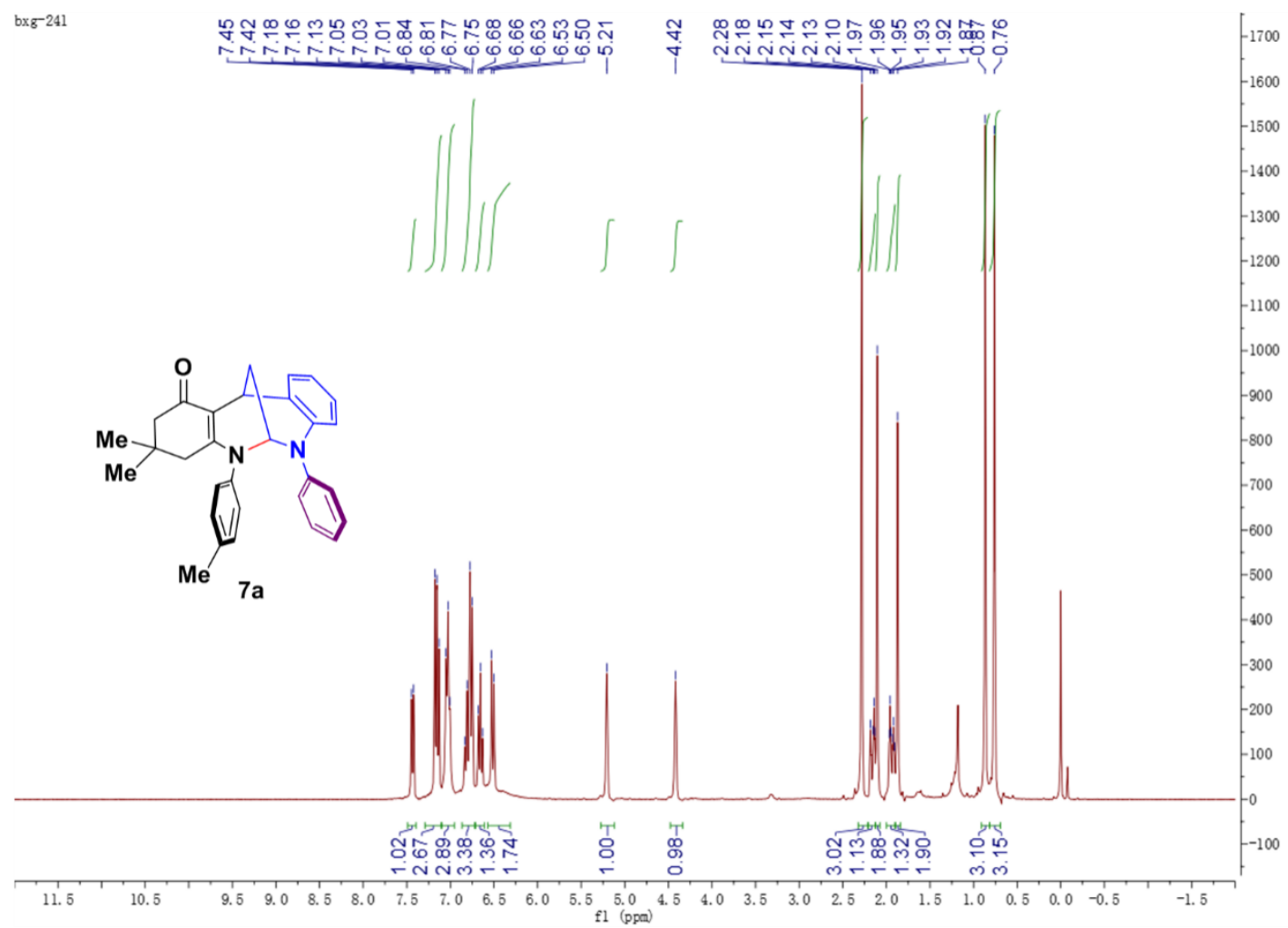

${ }^{13} \mathrm{C}$ NMR spectrum of $7 \mathbf{a}\left(100 \mathrm{MHz}, \mathrm{CDCl}_{3}\right)$ 


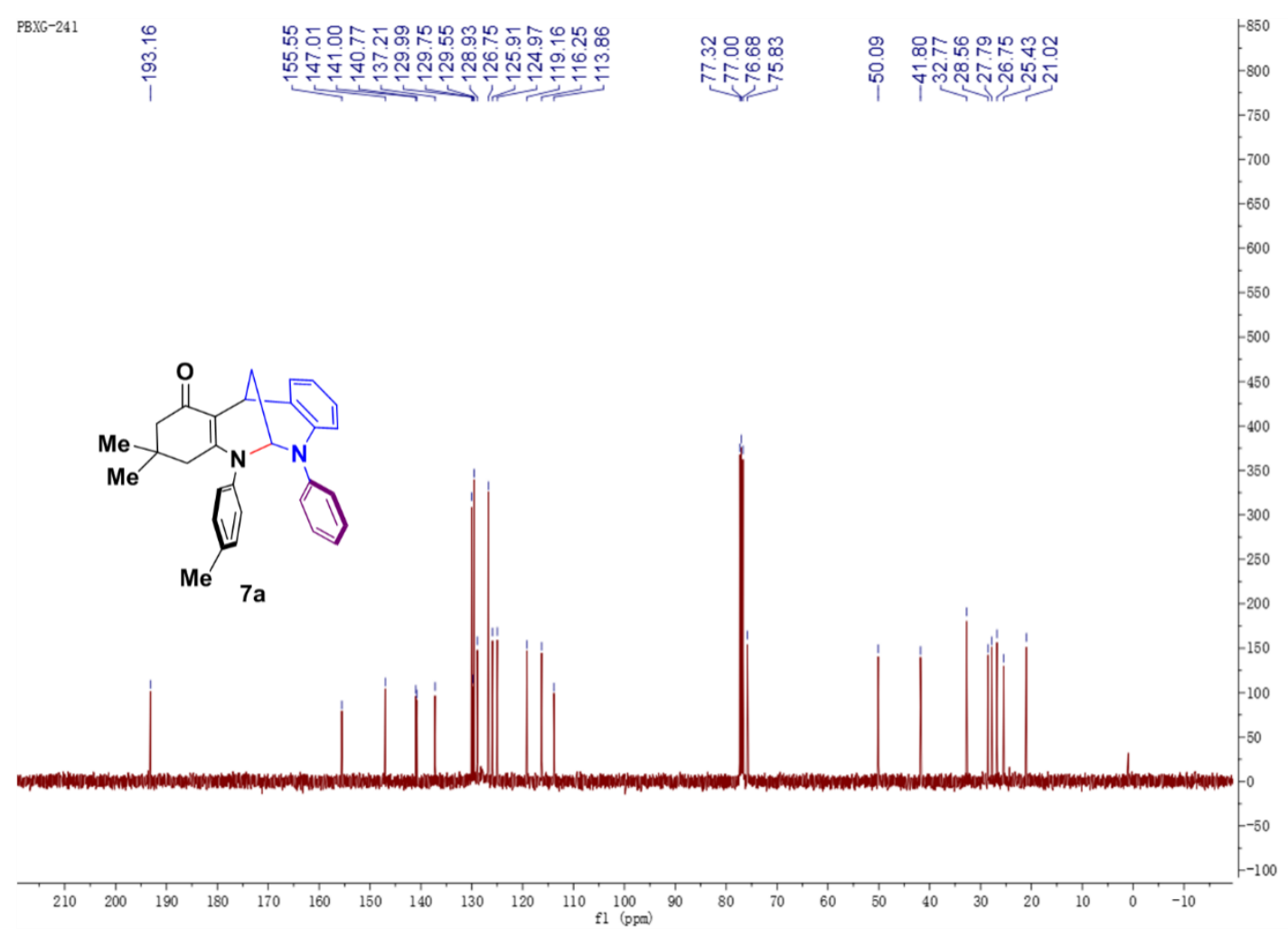

${ }^{1} \mathrm{H}$ NMR spectrum of $7 \mathbf{b}\left(400 \mathrm{MHz}, \mathrm{CDCl}_{3}\right)$

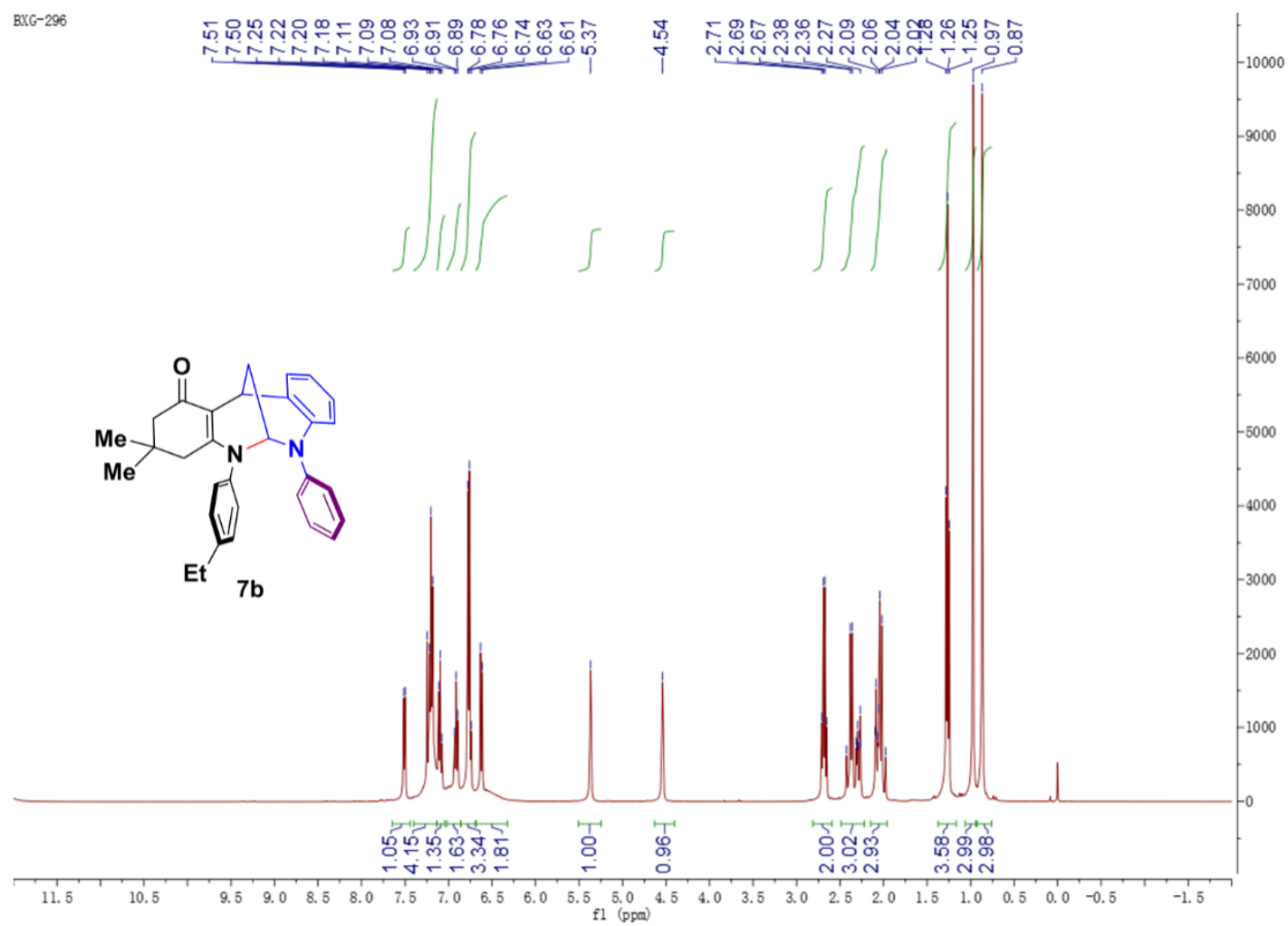

${ }^{13} \mathrm{C}$ NMR spectrum of $7 \mathbf{b}\left(100 \mathrm{MHz}, \mathrm{CDCl}_{3}\right)$ 


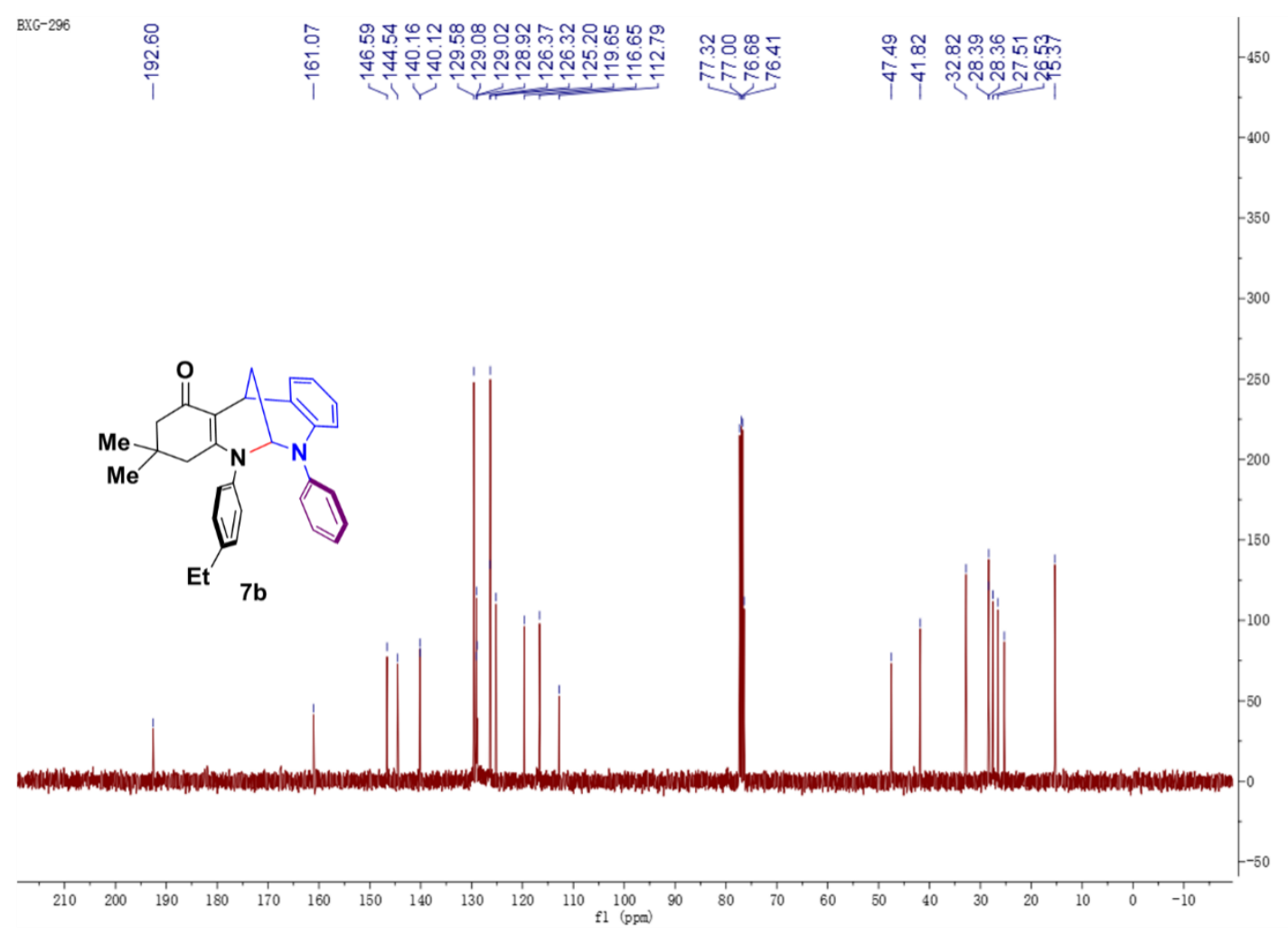

${ }^{1} \mathrm{H}$ NMR spectrum of $\mathbf{7 c}\left(400 \mathrm{MHz}, \mathrm{CDCl}_{3}\right)$

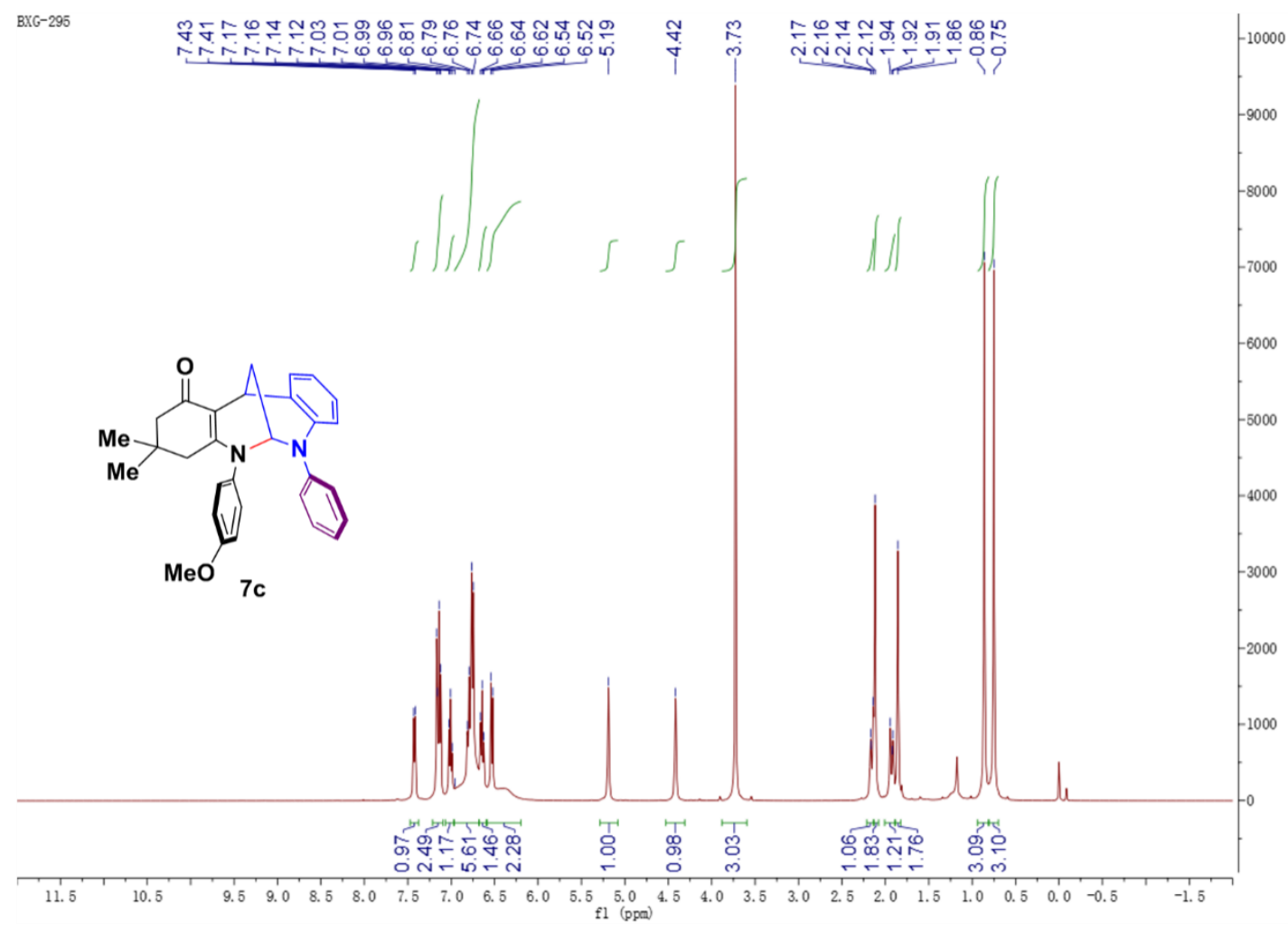

${ }^{13} \mathrm{C}$ NMR spectrum of $\mathbf{7 c}\left(100 \mathrm{MHz}, \mathrm{CDCl}_{3}\right)$ 


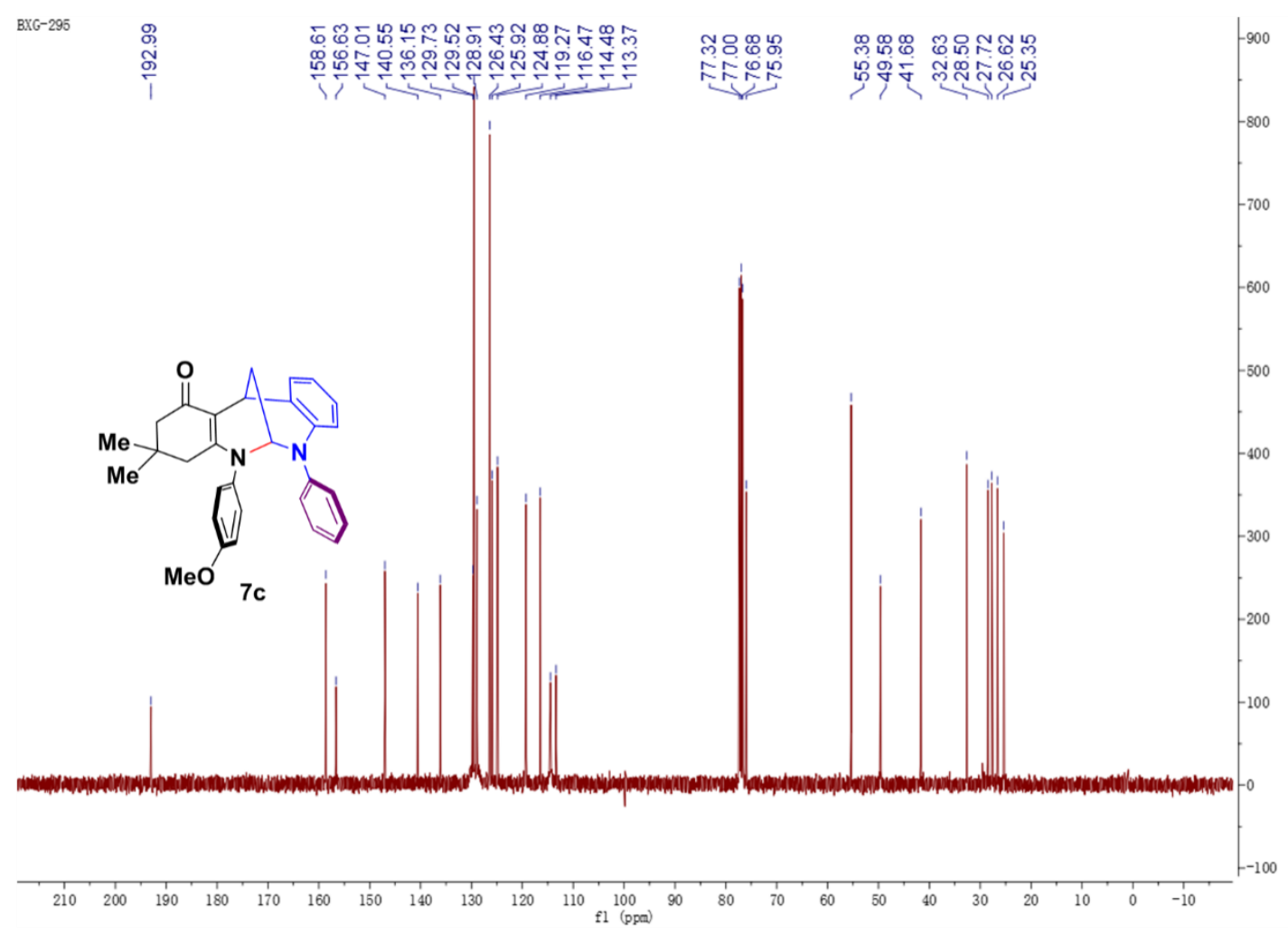

${ }^{1} \mathrm{H}$ NMR spectrum of $7 \mathbf{d}\left(400 \mathrm{MHz}, \mathrm{CDCl}_{3}\right)$

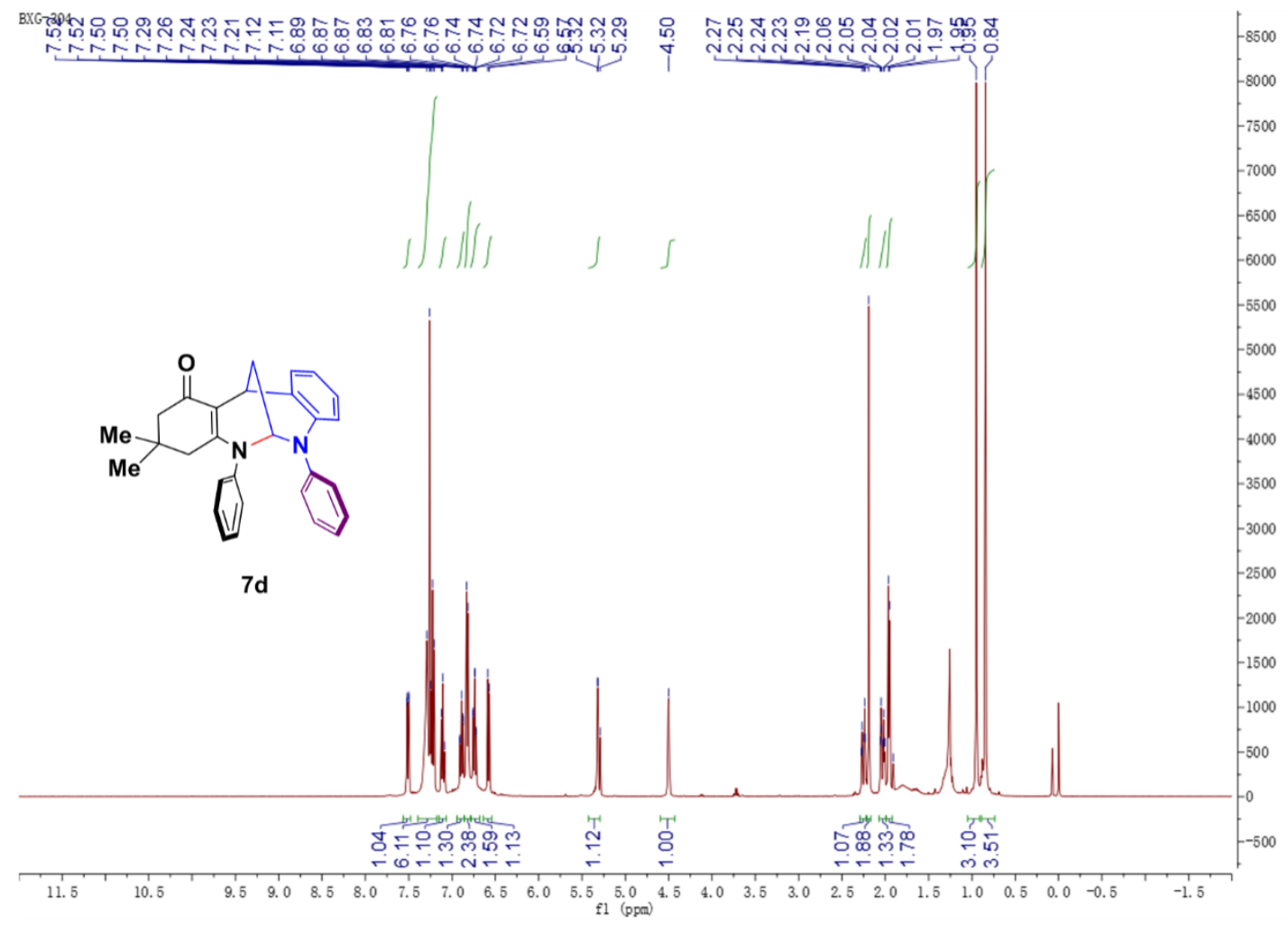

${ }^{13} \mathrm{C}$ NMR spectrum of $7 \mathbf{d}\left(100 \mathrm{MHz}, \mathrm{CDCl}_{3}\right)$ 


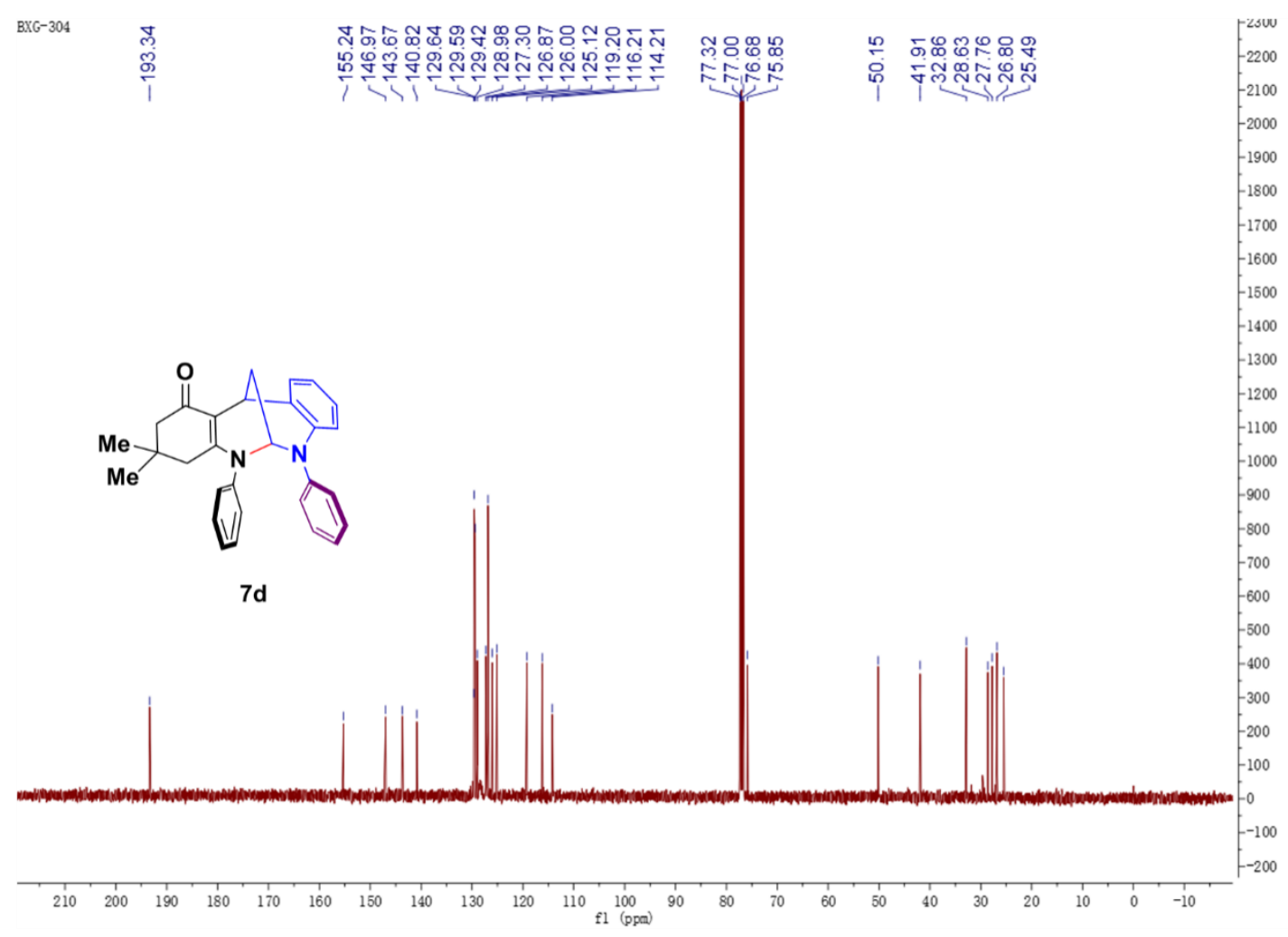

${ }^{1} \mathrm{H}$ NMR spectrum of $\mathbf{7 e}\left(400 \mathrm{MHz}, \mathrm{CDCl}_{3}\right)$

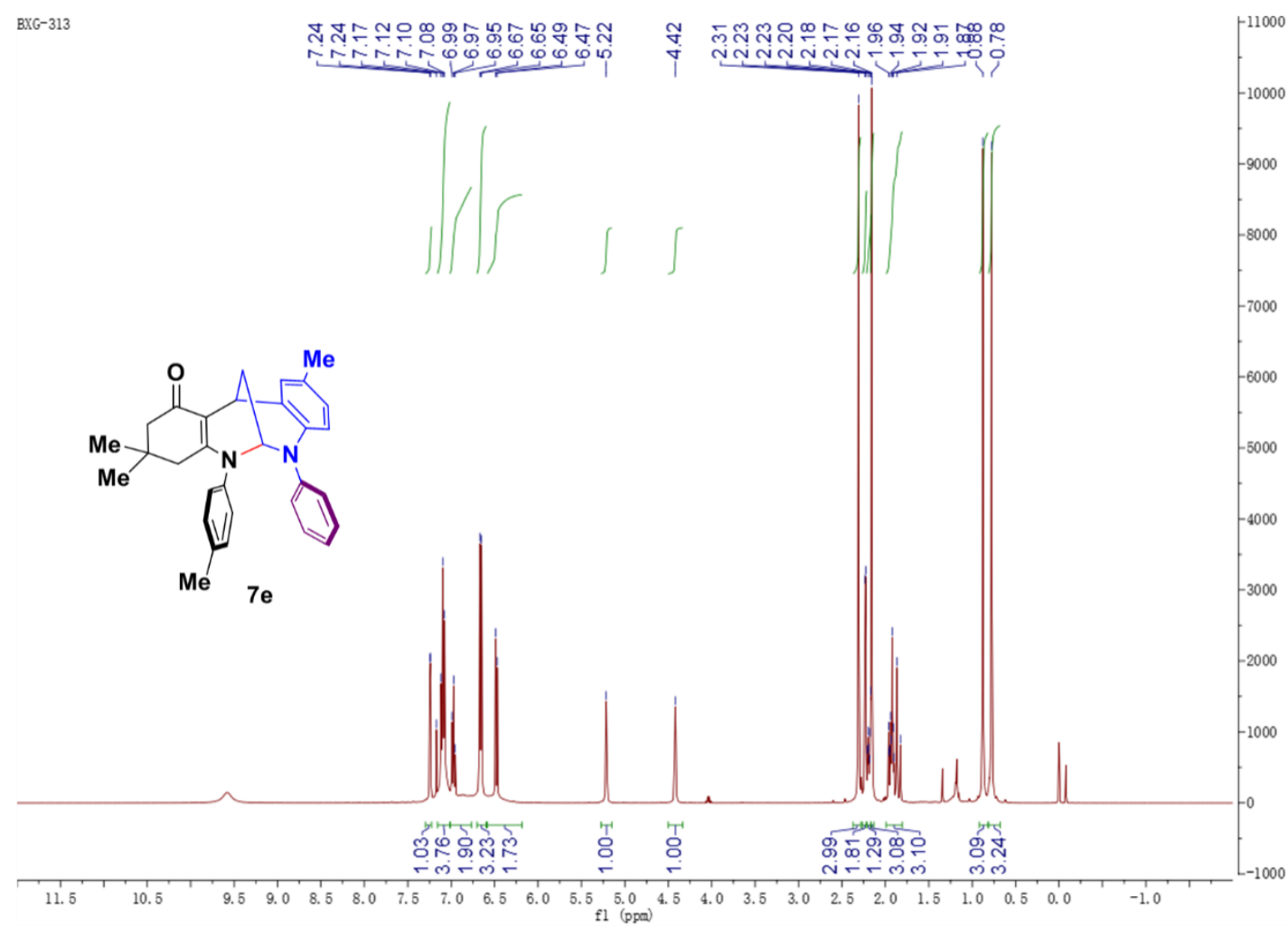

${ }^{13} \mathrm{C}$ NMR spectrum of $7 \mathbf{e}\left(100 \mathrm{MHz}, \mathrm{CDCl}_{3}\right)$ 


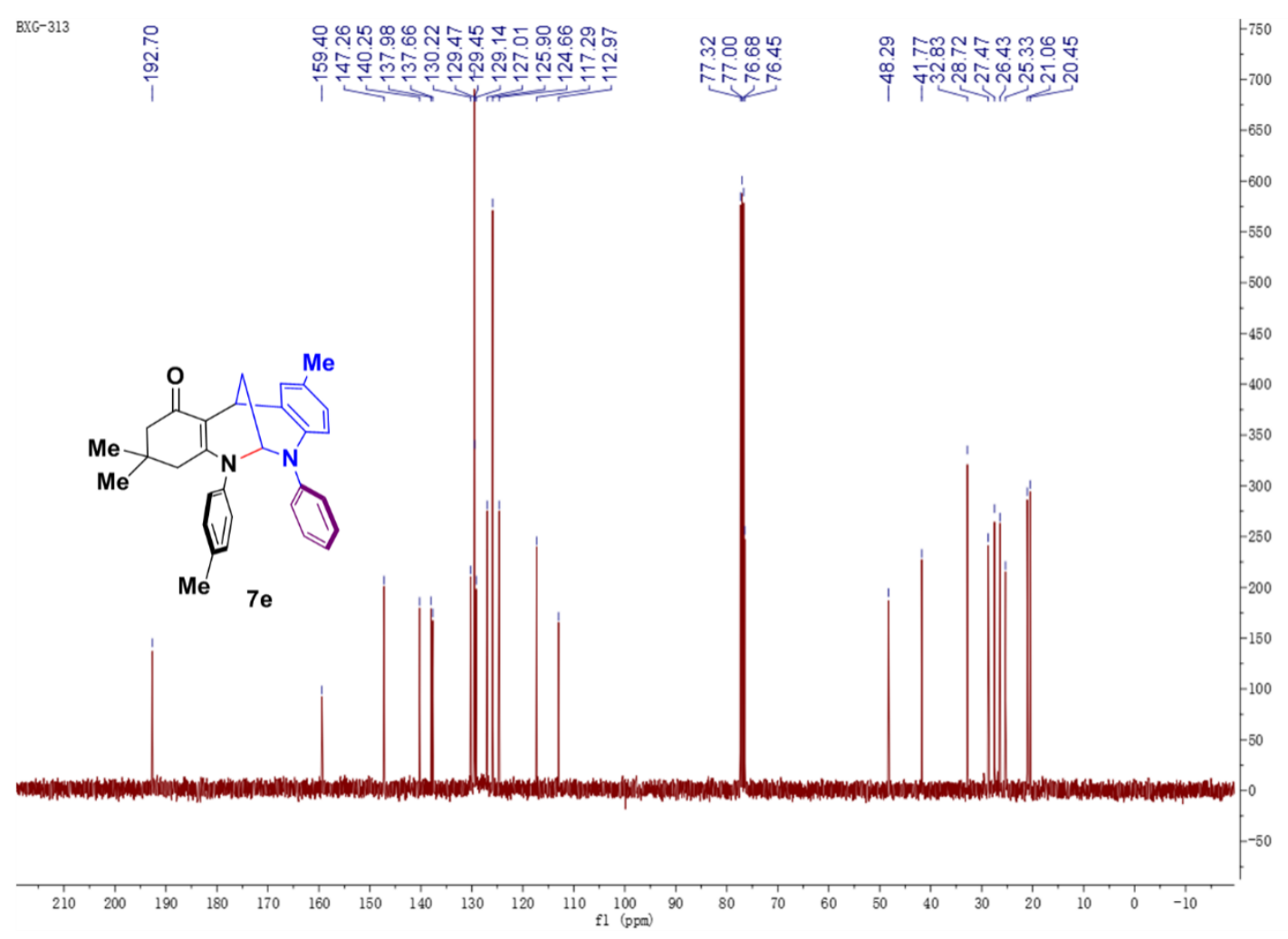

${ }^{1} \mathrm{H}$ NMR spectrum of $7 \mathbf{f}\left(400 \mathrm{MHz}, \mathrm{CDCl}_{3}\right)$

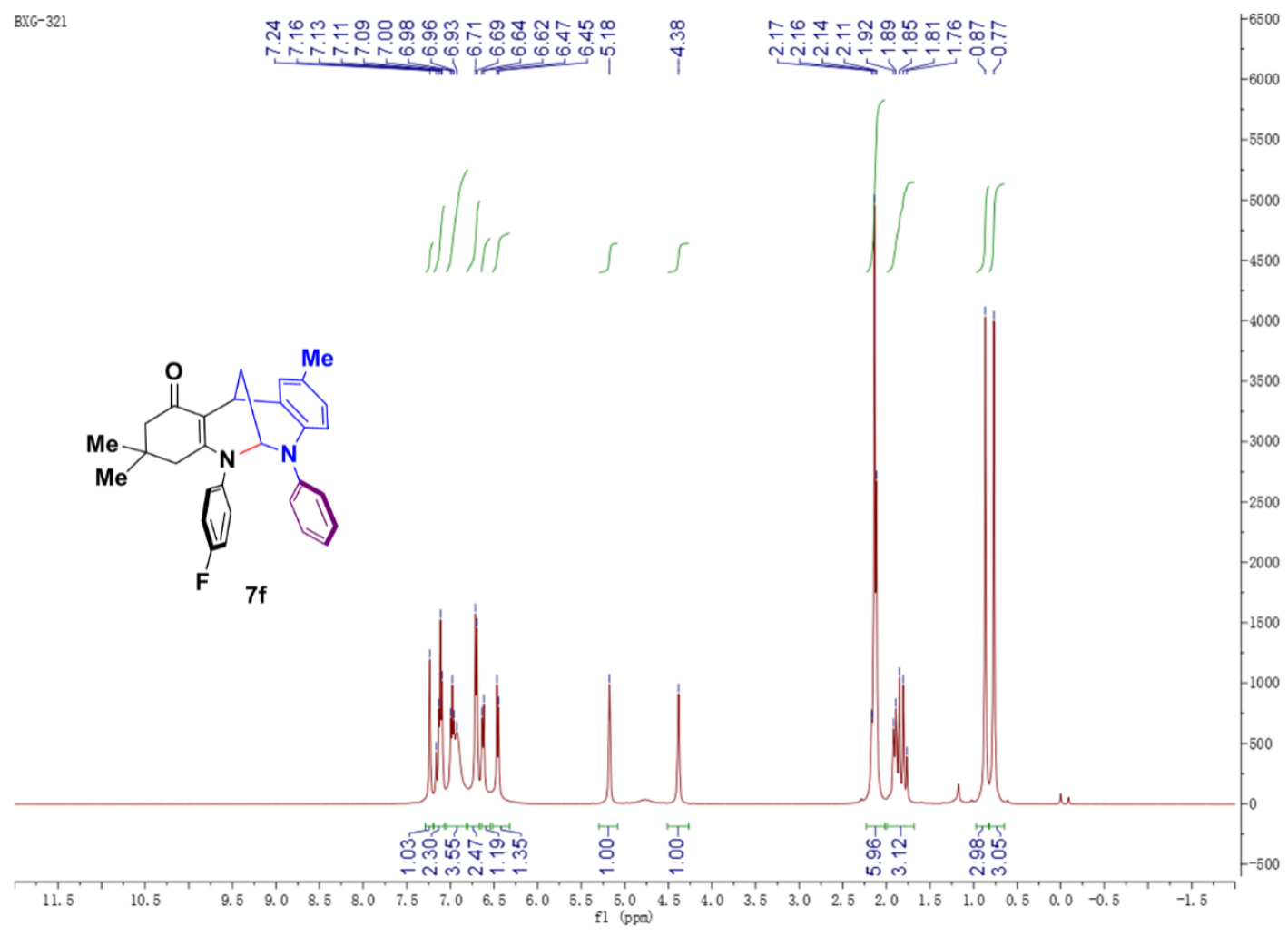

${ }^{13} \mathrm{C}$ NMR spectrum of $7 \mathbf{f}\left(100 \mathrm{MHz}, \mathrm{CDCl}_{3}\right)$ 


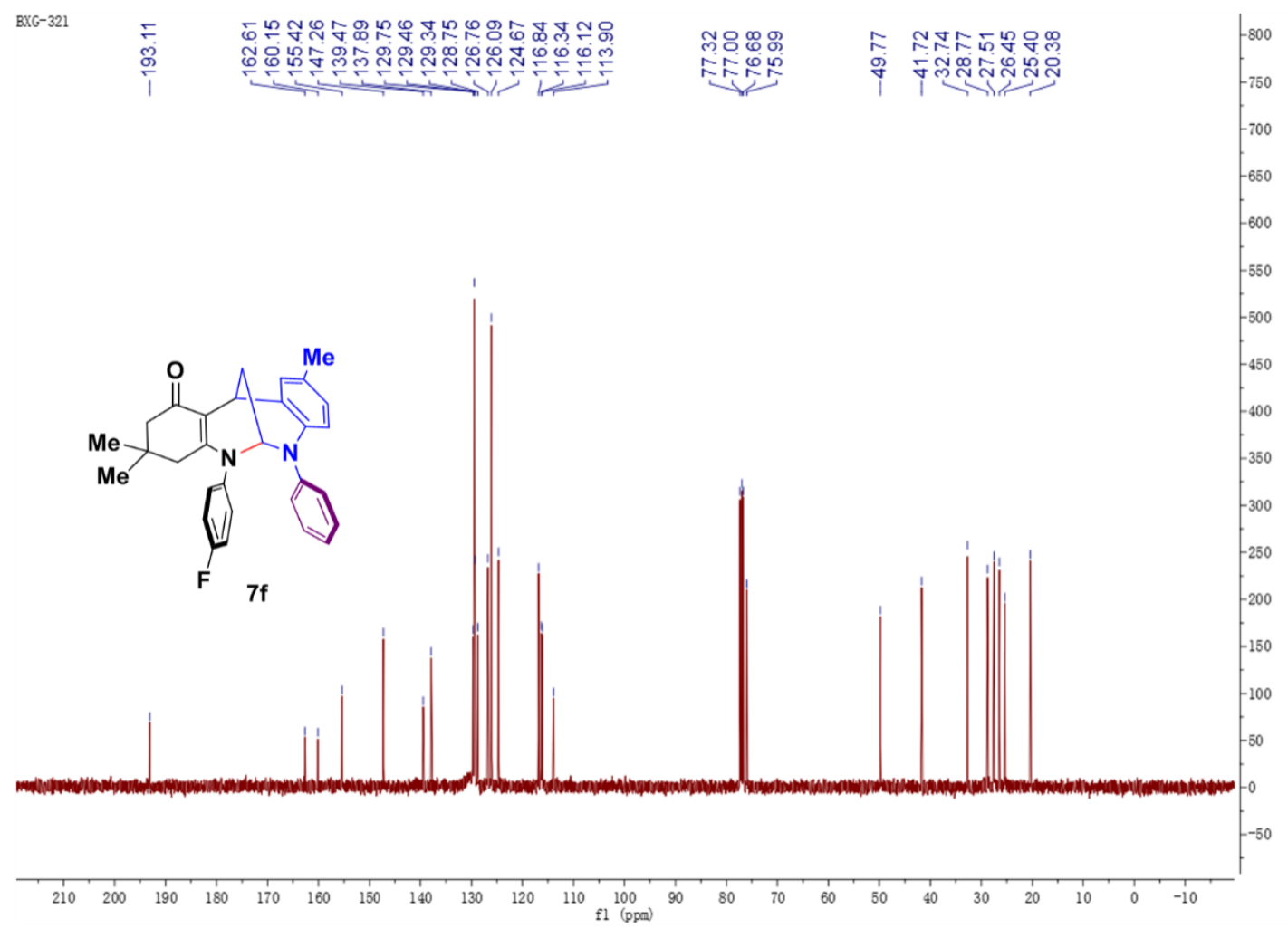

${ }^{1} \mathrm{H}$ NMR spectrum of $\mathbf{7 g}\left(400 \mathrm{MHz}, \mathrm{CDCl}_{3}\right)$

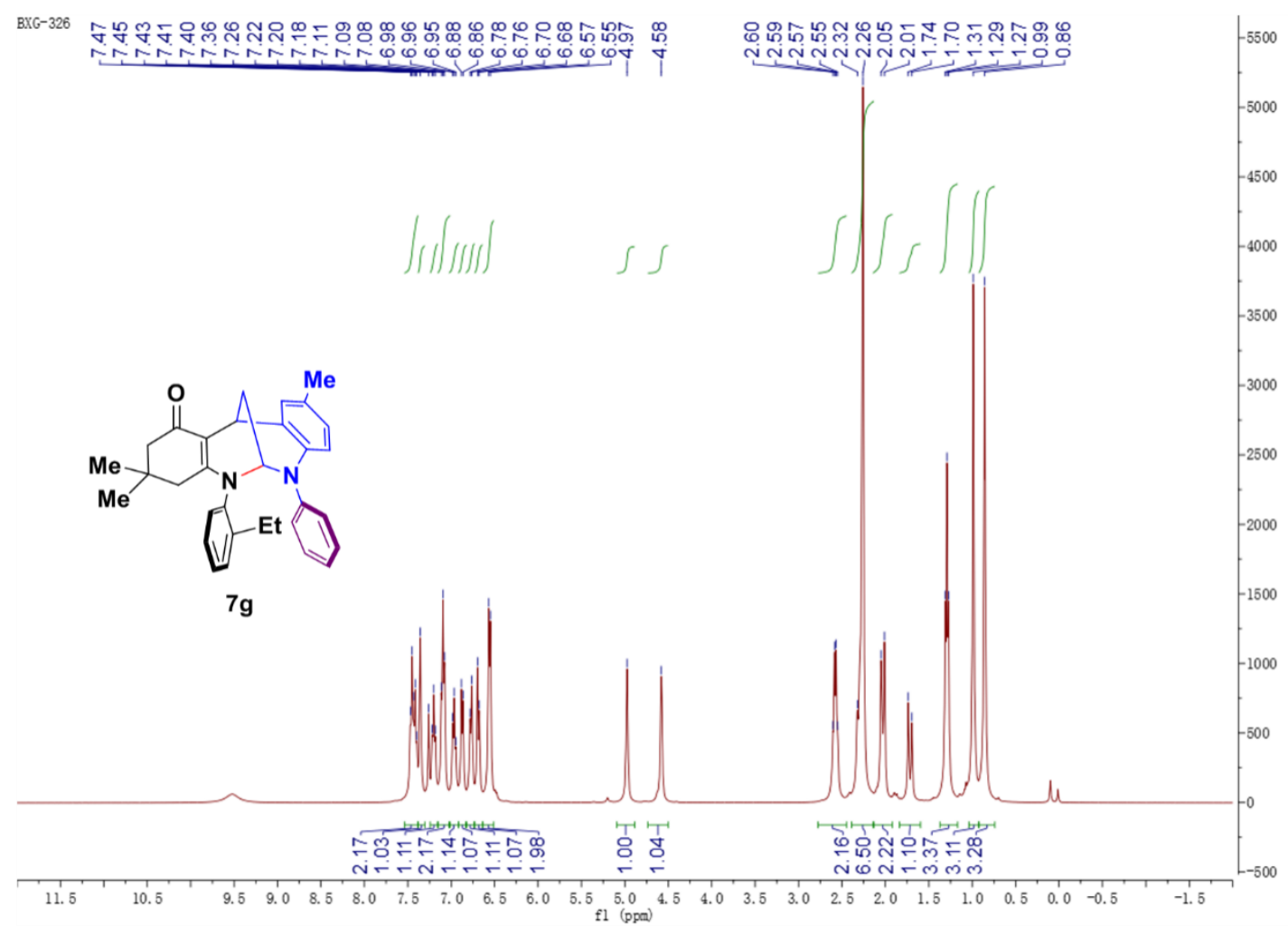

${ }^{13} \mathrm{C}$ NMR spectrum of $7 \mathbf{g}\left(100 \mathrm{MHz}, \mathrm{CDCl}_{3}\right)$ 


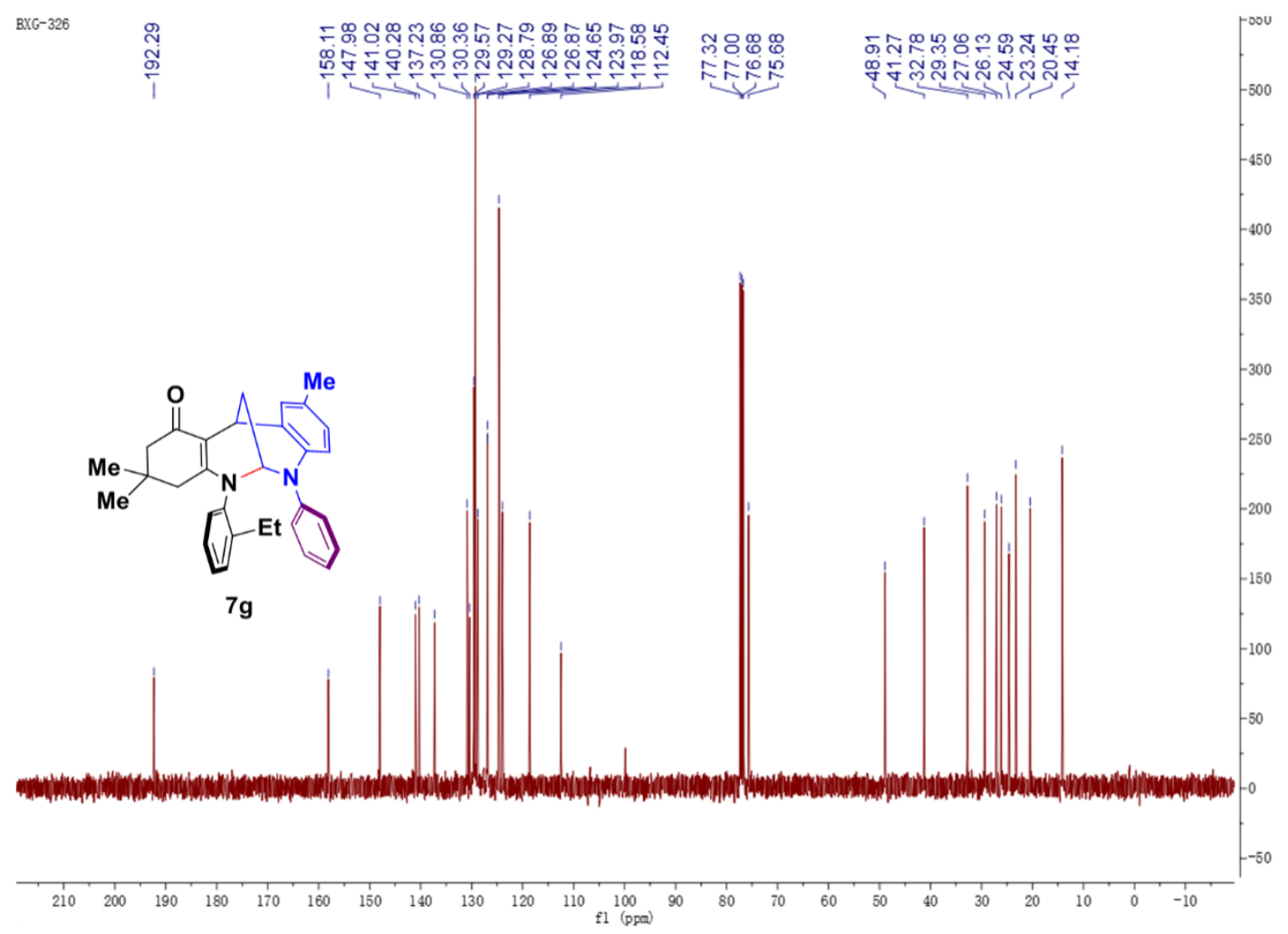

${ }^{1} \mathrm{H}$ NMR spectrum of $\mathbf{7 h}\left(400 \mathrm{MHz}, \mathrm{CDCl}_{3}\right)$

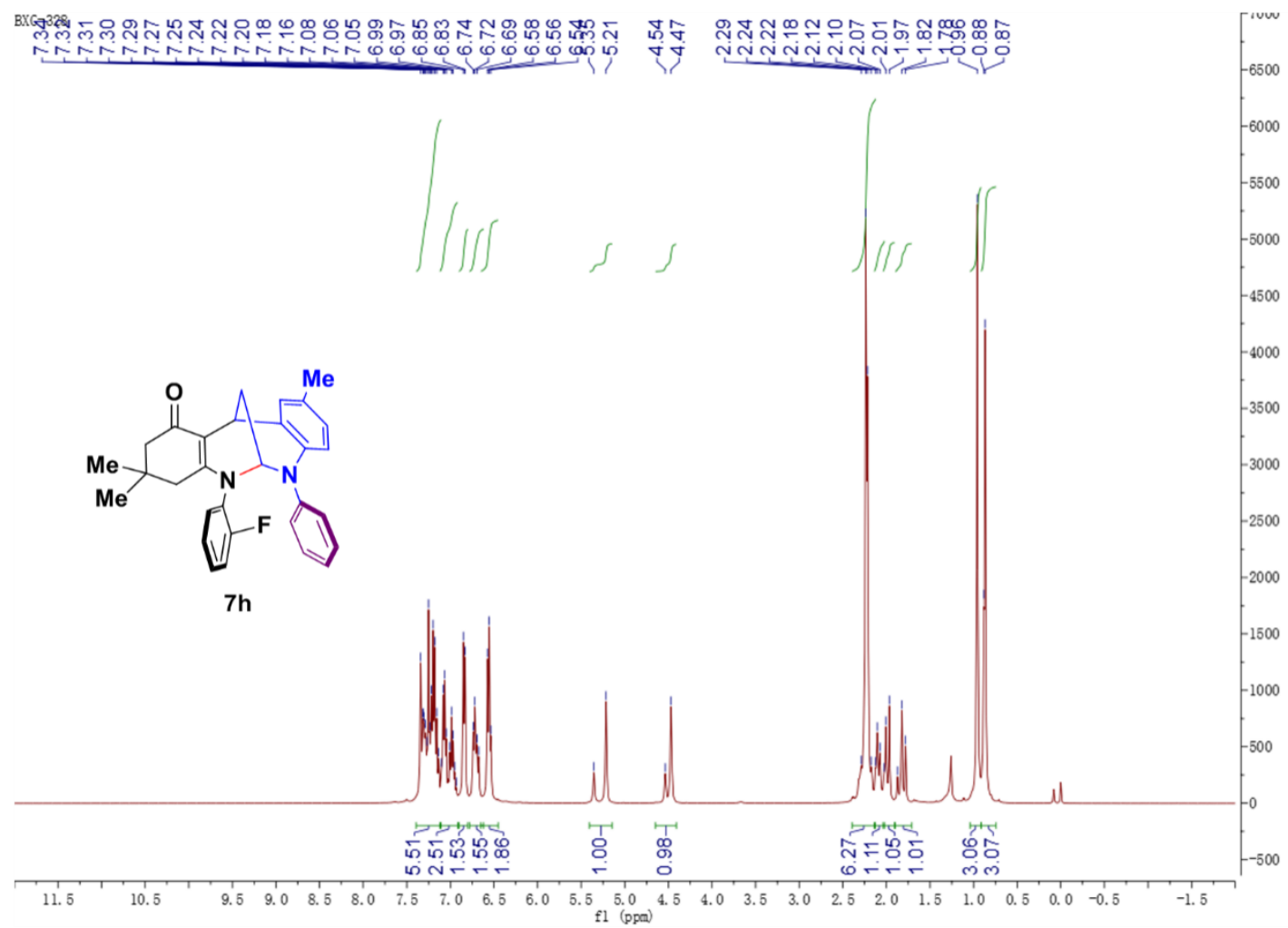

${ }^{13} \mathrm{C}$ NMR spectrum of $7 \mathbf{h}\left(100 \mathrm{MHz}, \mathrm{CDCl}_{3}\right)$ 


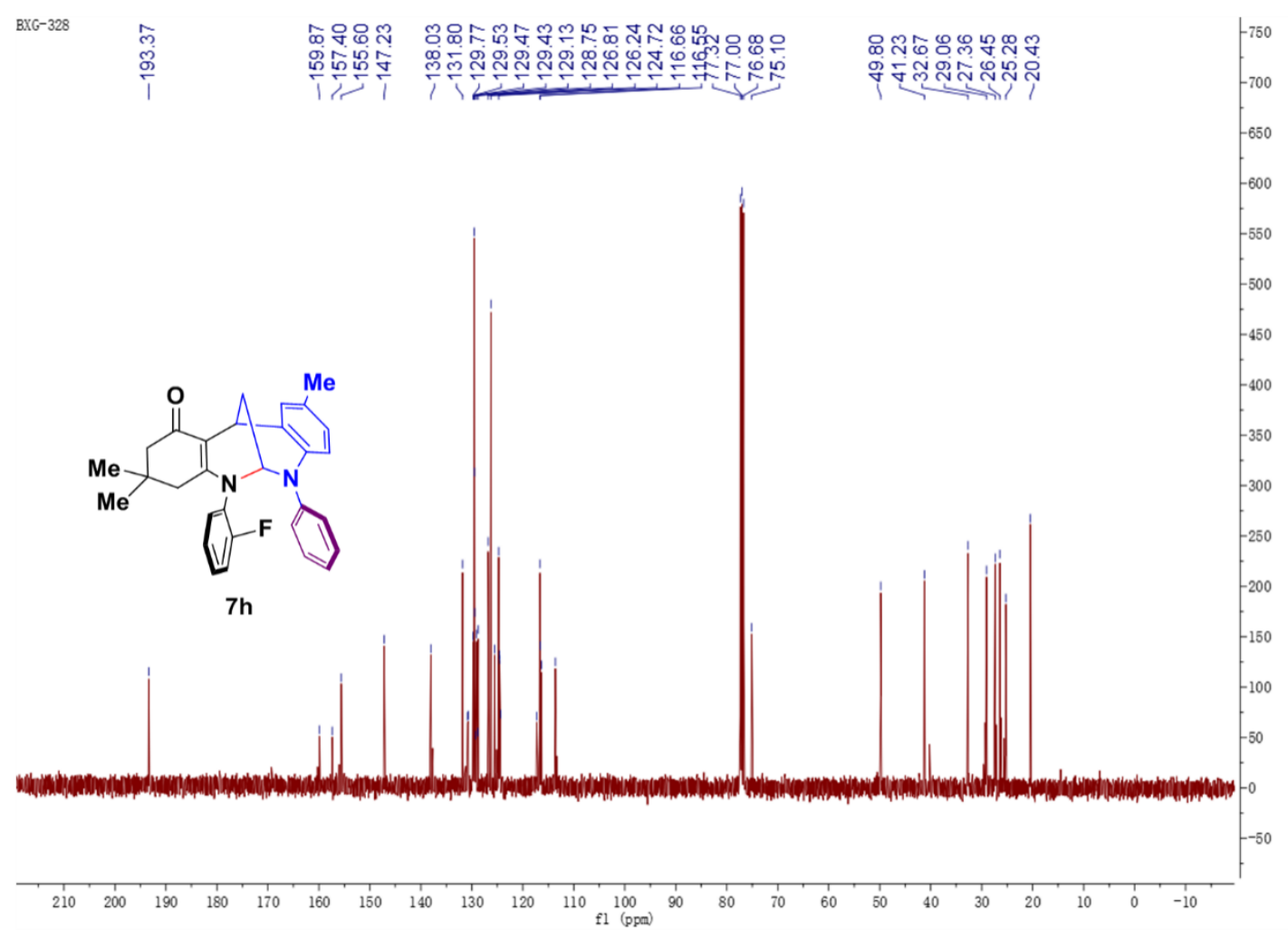

${ }^{1} \mathrm{H}$ NMR spectrum of $\mathbf{7 i}\left(400 \mathrm{MHz}, \mathrm{CDCl}_{3}\right)$

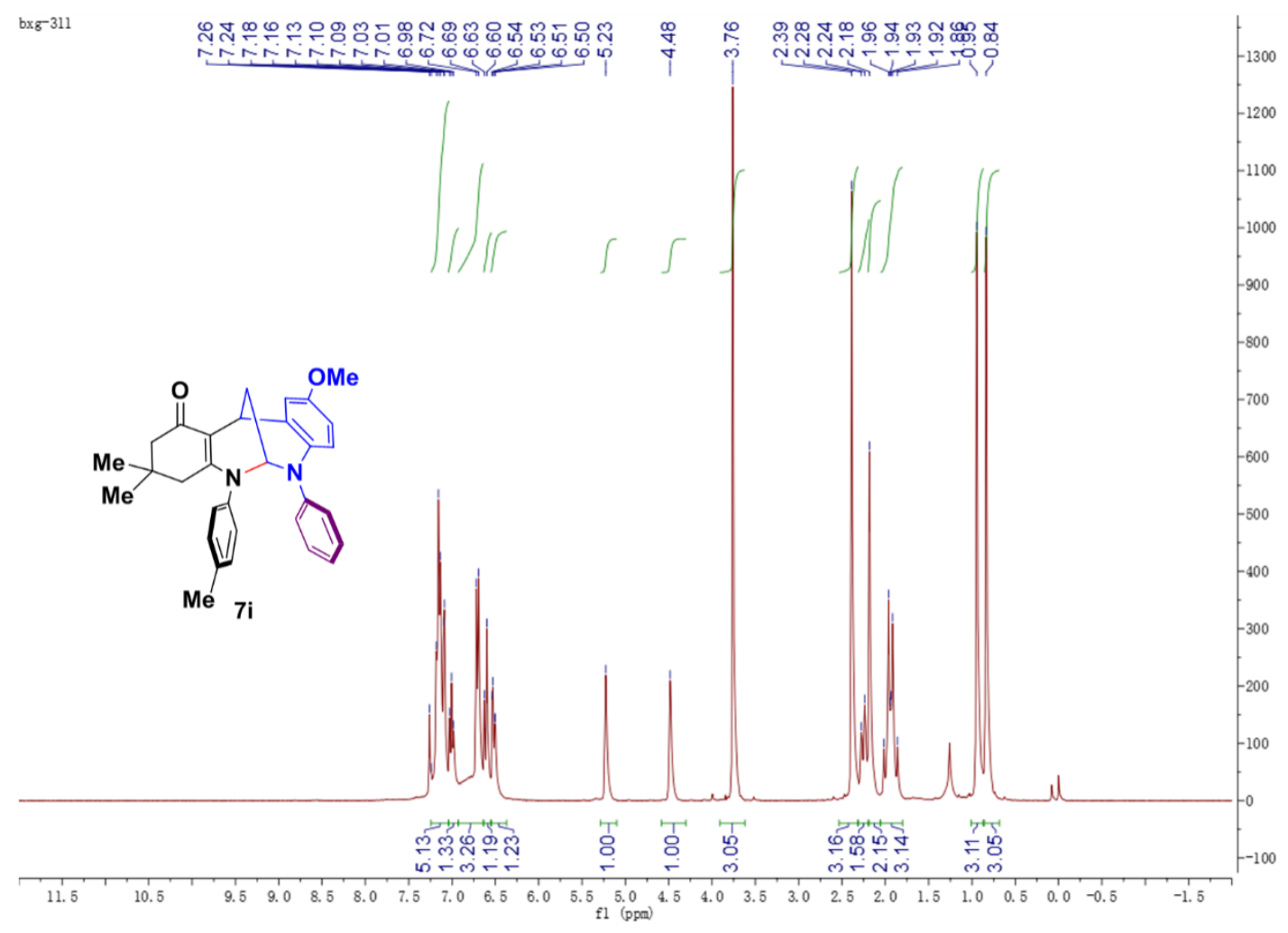

${ }^{13} \mathrm{C}$ NMR spectrum of $7 \mathbf{i}\left(100 \mathrm{MHz}, \mathrm{CDCl}_{3}\right)$ 


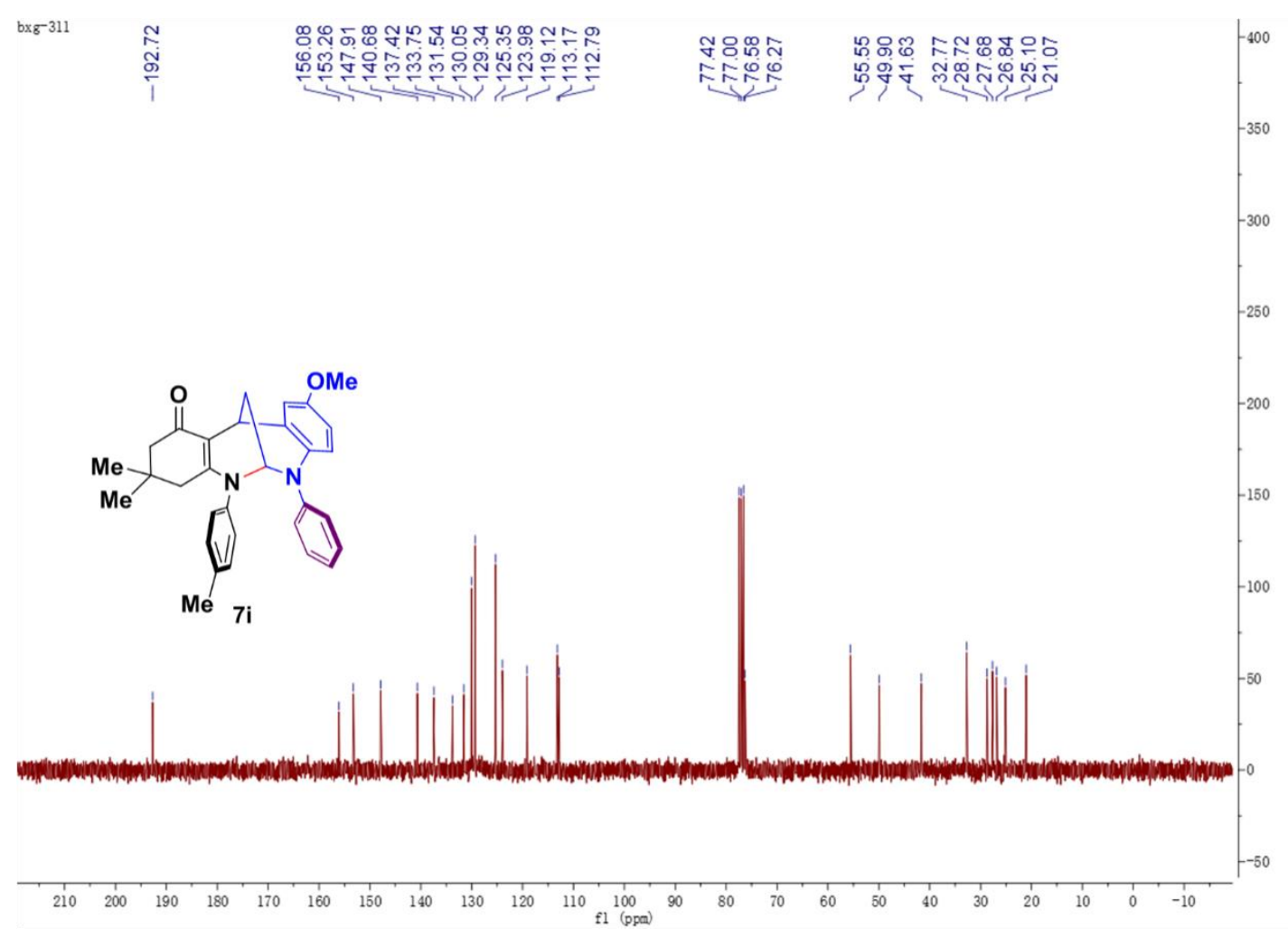

${ }^{1} \mathrm{H}$ NMR spectrum of $\mathbf{7 j}\left(400 \mathrm{MHz}, \mathrm{CDCl}_{3}\right)$

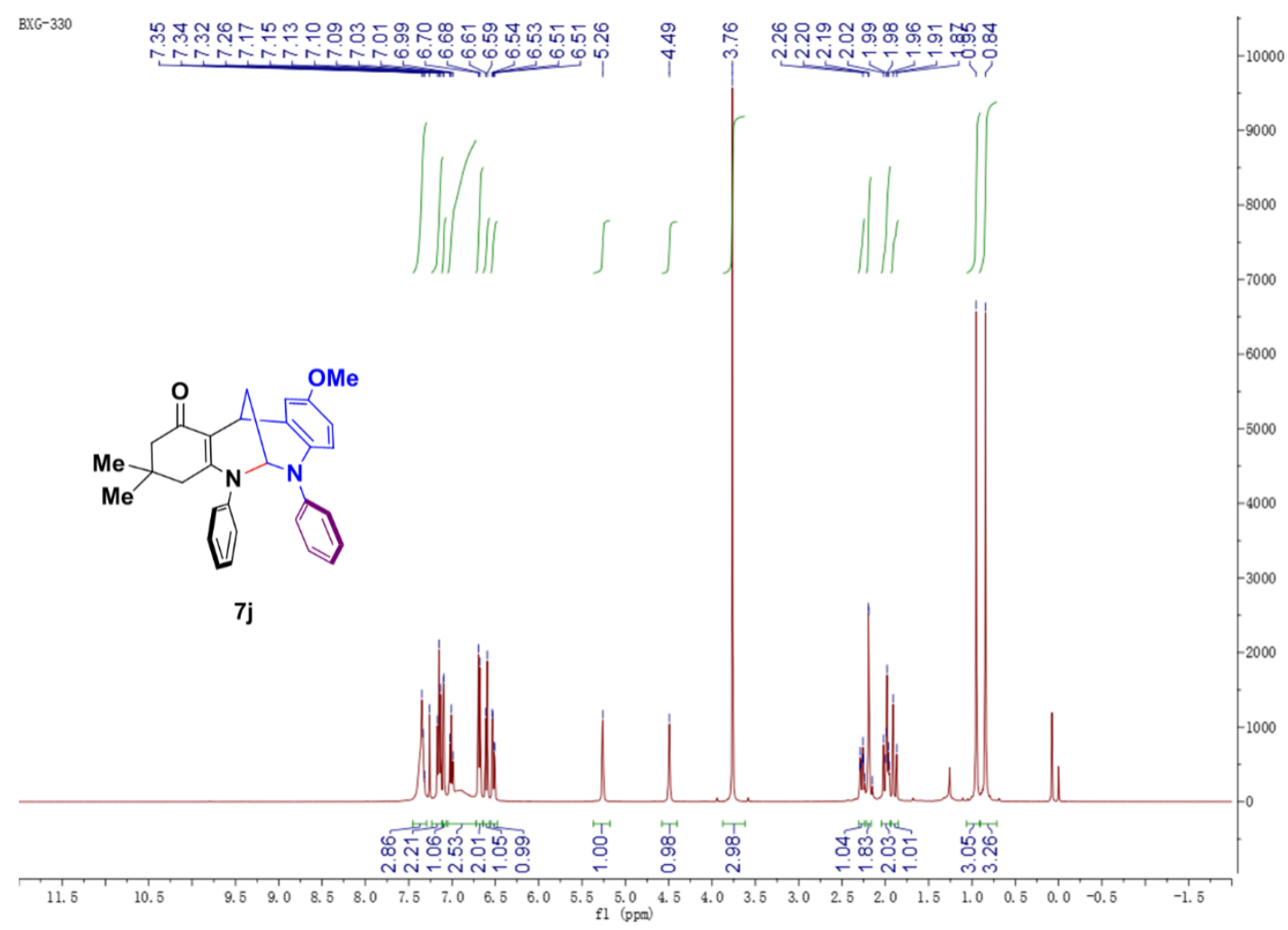

${ }^{13} \mathrm{C}$ NMR spectrum of $\mathbf{7 j}\left(100 \mathrm{MHz}, \mathrm{CDCl}_{3}\right)$ 


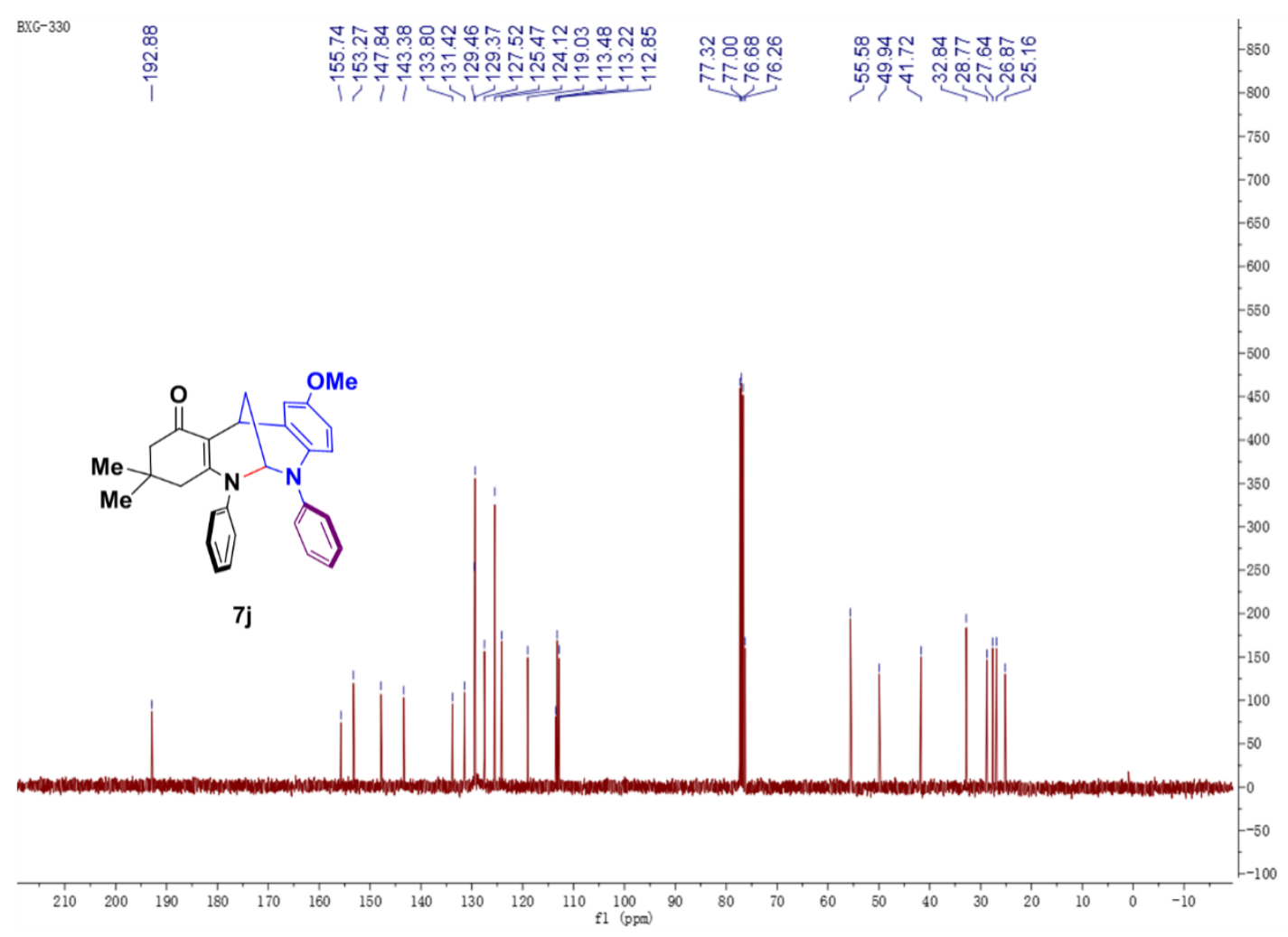

${ }^{1} \mathrm{H}$ NMR spectrum of $7 \mathbf{k}\left(400 \mathrm{MHz}, \mathrm{CDCl}_{3}\right)$

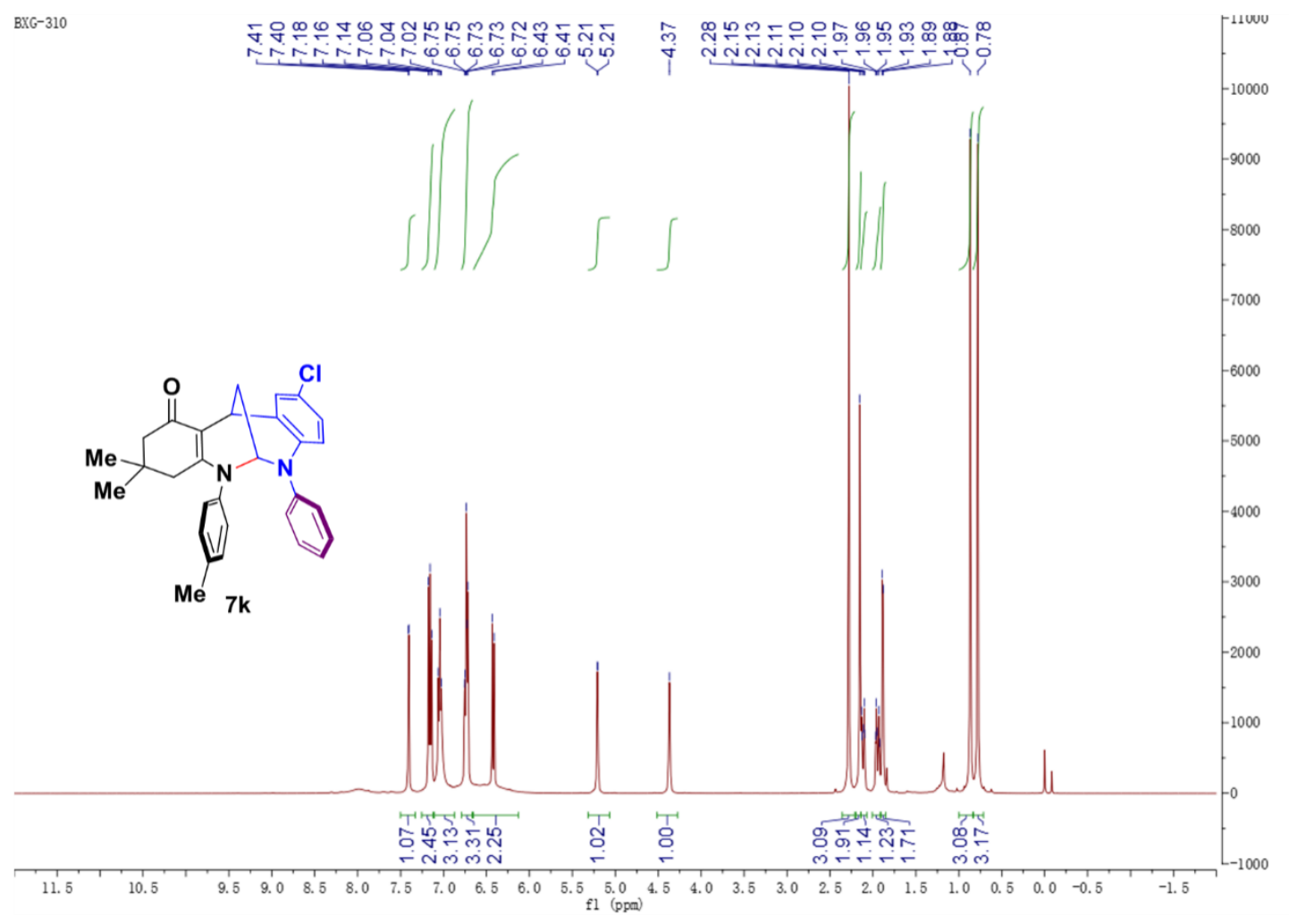

${ }^{13} \mathrm{C}$ NMR spectrum of $7 \mathbf{k}\left(100 \mathrm{MHz}, \mathrm{CDCl}_{3}\right)$ 


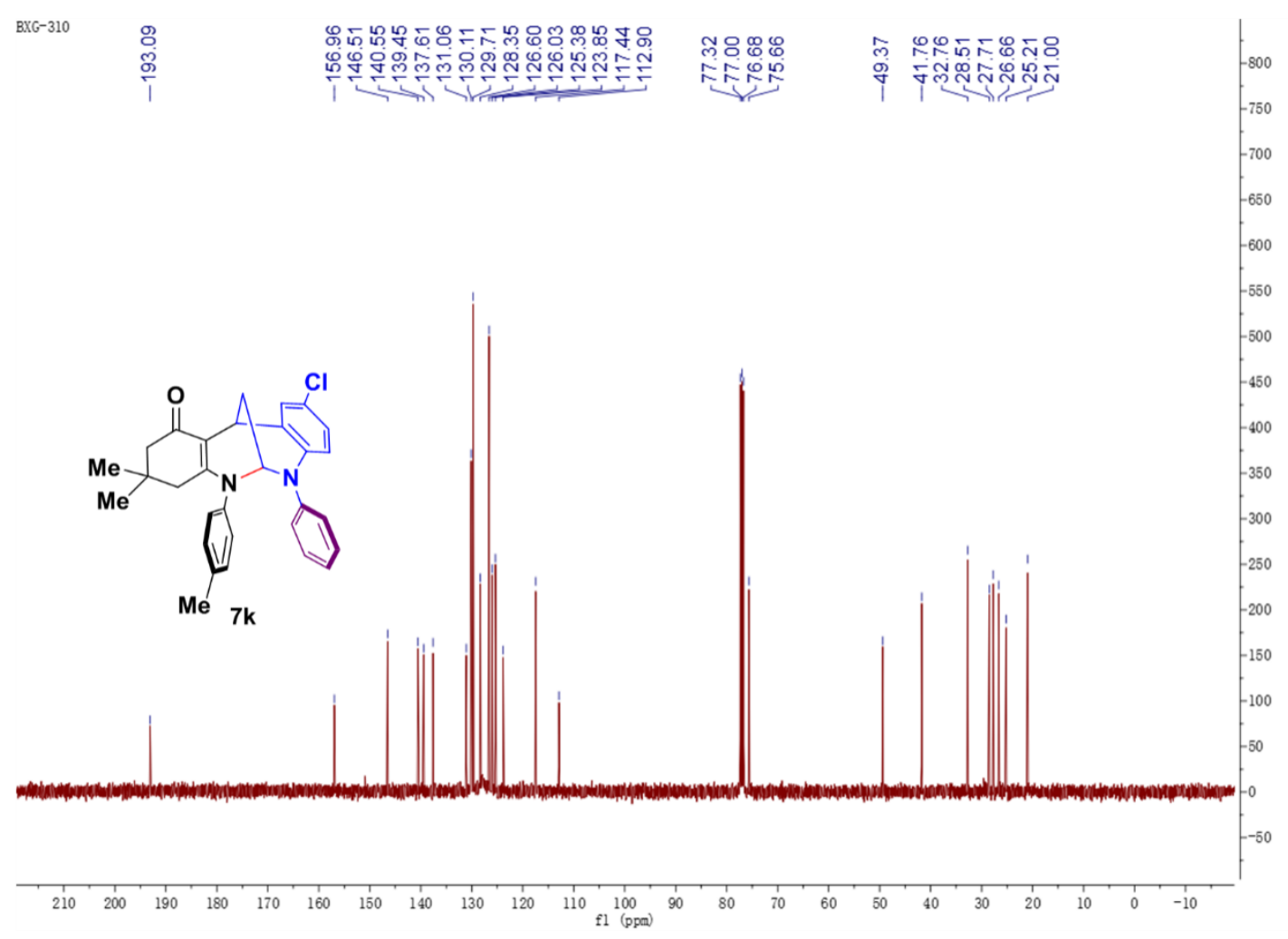

${ }^{1} \mathrm{H}$ NMR spectrum of $7 \mathbf{l}\left(400 \mathrm{MHz}, \mathrm{CDCl}_{3}\right)$

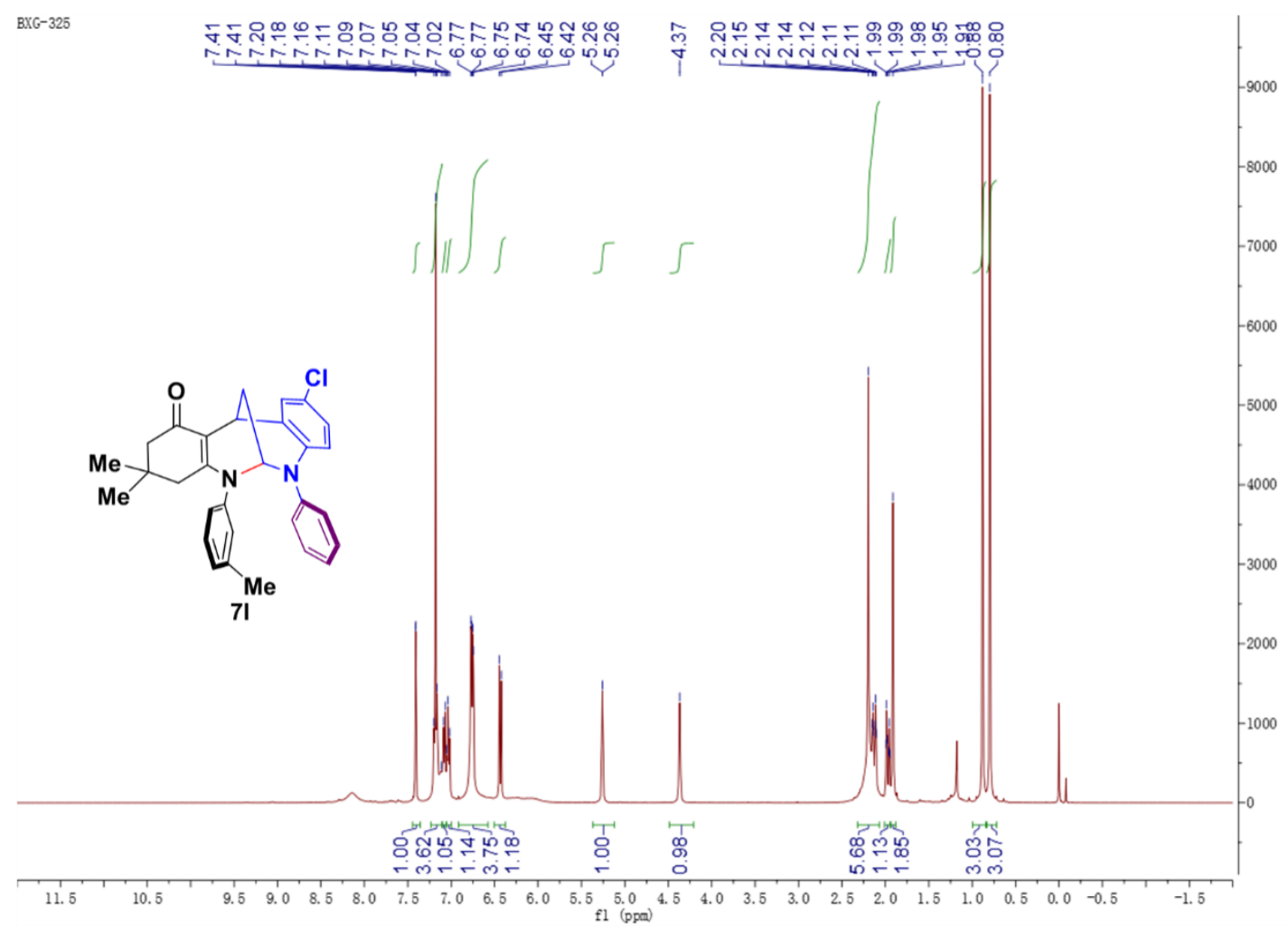

${ }^{13} \mathrm{C}$ NMR spectrum of $7 \mathbf{l}\left(100 \mathrm{MHz}, \mathrm{CDCl}_{3}\right)$ 


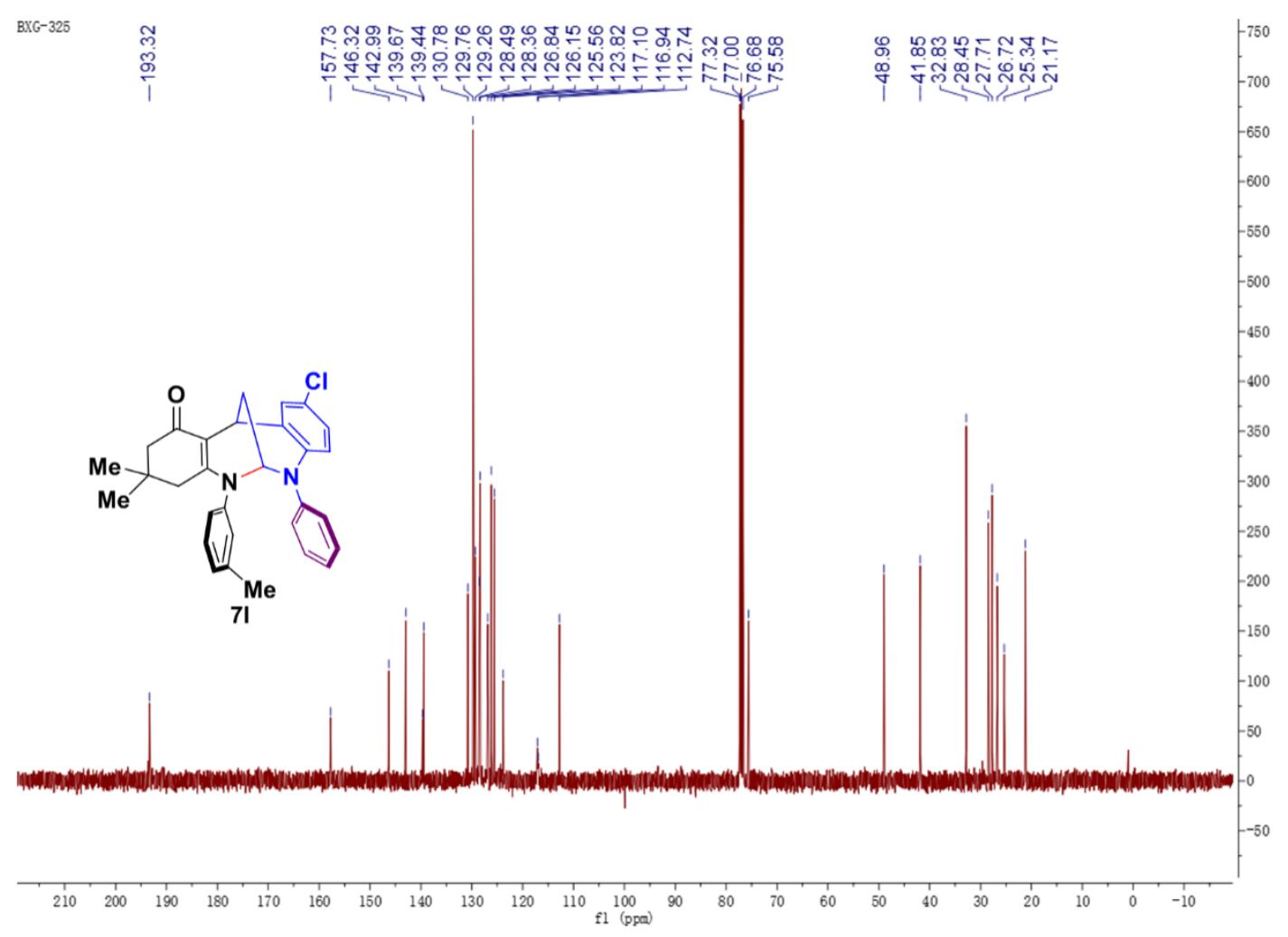

${ }^{1} \mathrm{H}$ NMR spectrum of $\mathbf{7 m}\left(400 \mathrm{MHz}, \mathrm{CDCl}_{3}\right)$

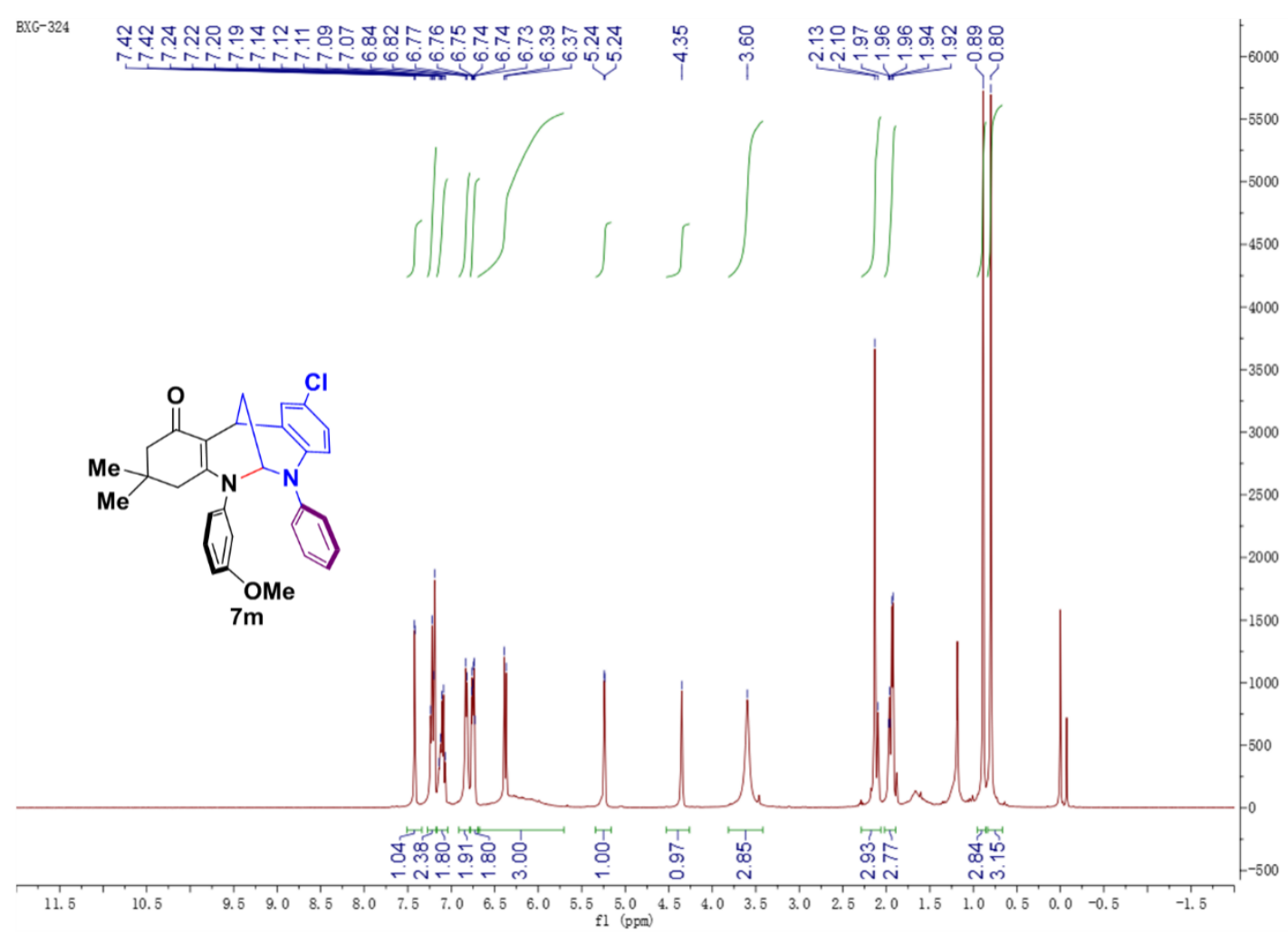

${ }^{13} \mathrm{C}$ NMR spectrum of $7 \mathbf{m}\left(100 \mathrm{MHz}, \mathrm{CDCl}_{3}\right)$ 


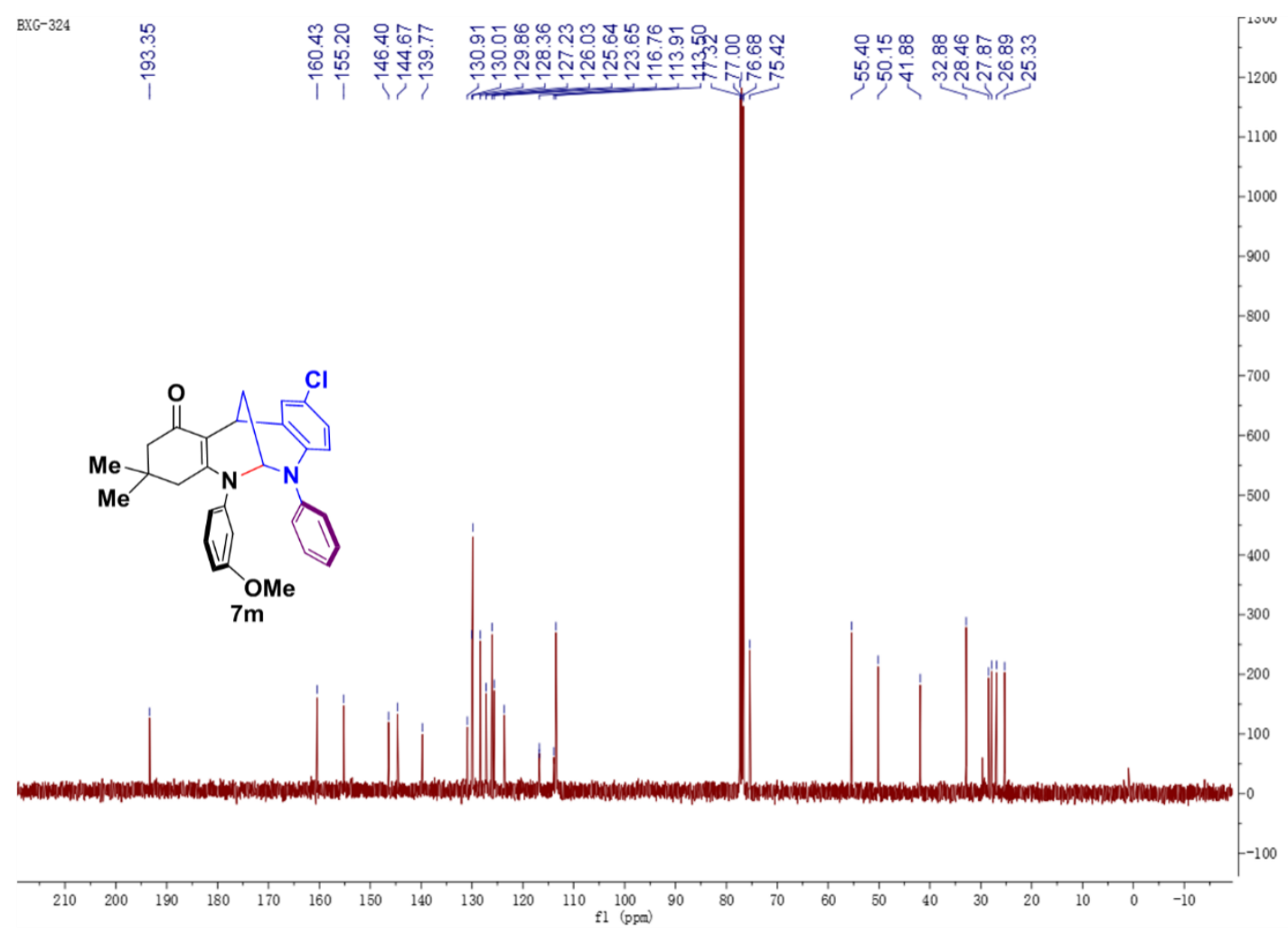

${ }^{1} \mathrm{H}$ NMR spectrum of $\mathbf{7 n}\left(400 \mathrm{MHz}, \mathrm{CDCl}_{3}\right)$

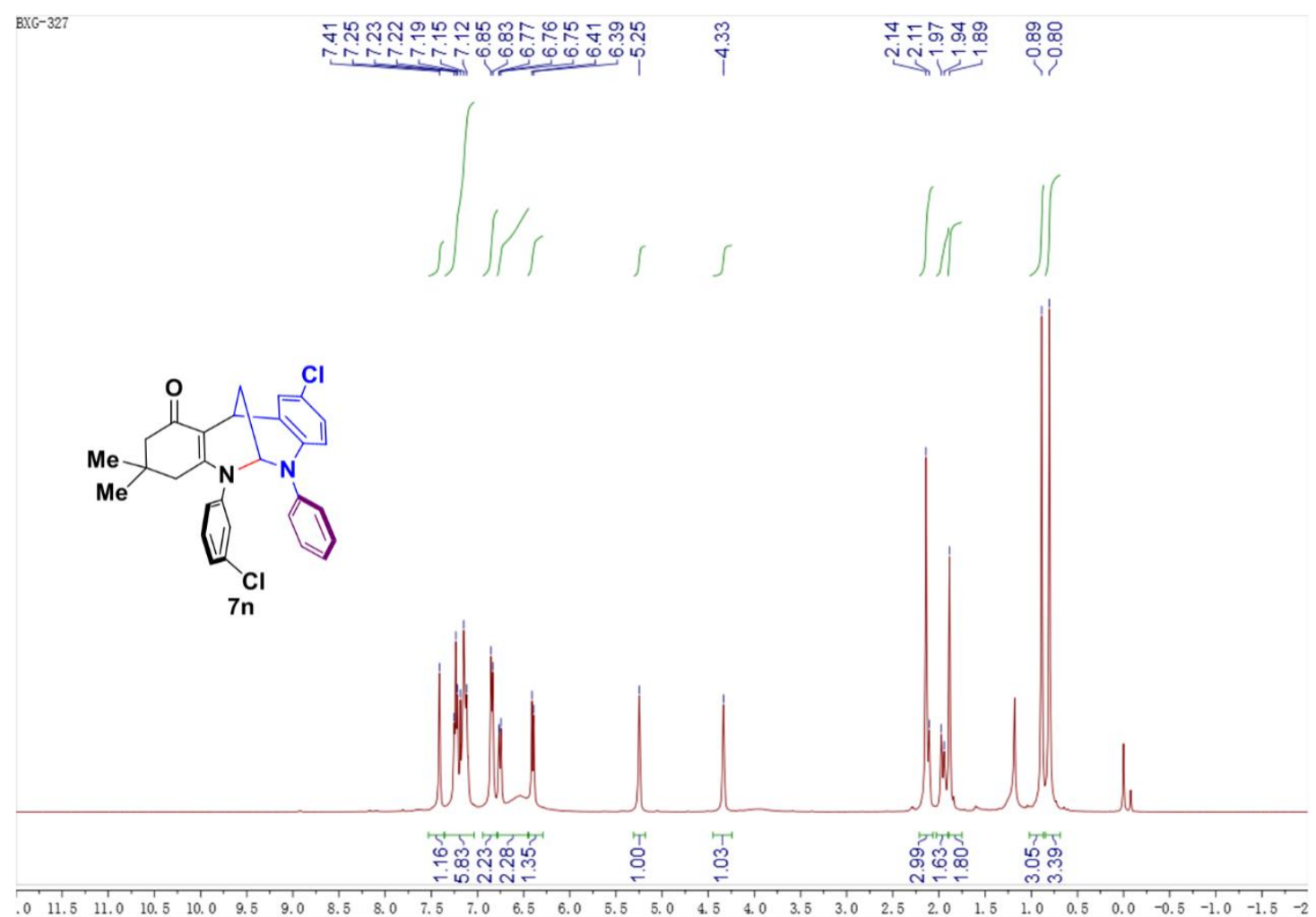

${ }^{13} \mathrm{C}$ NMR spectrum of $7 \mathbf{n}\left(100 \mathrm{MHz}, \mathrm{CDCl}_{3}\right)$ 


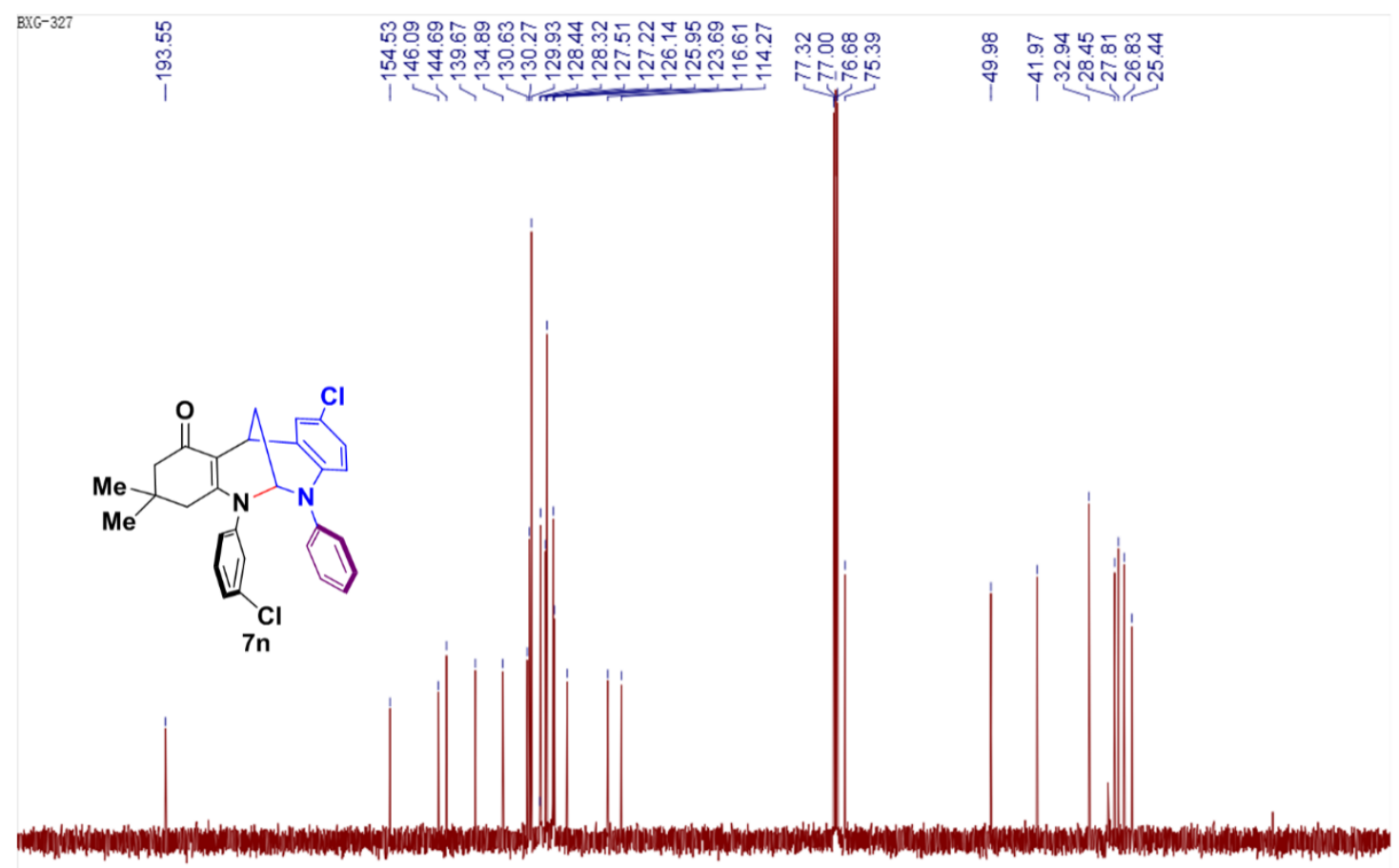

${ }^{1} \mathrm{H}$ NMR spectrum of $7 \mathbf{o}\left(400 \mathrm{MHz}, \mathrm{CDCl}_{3}\right)$

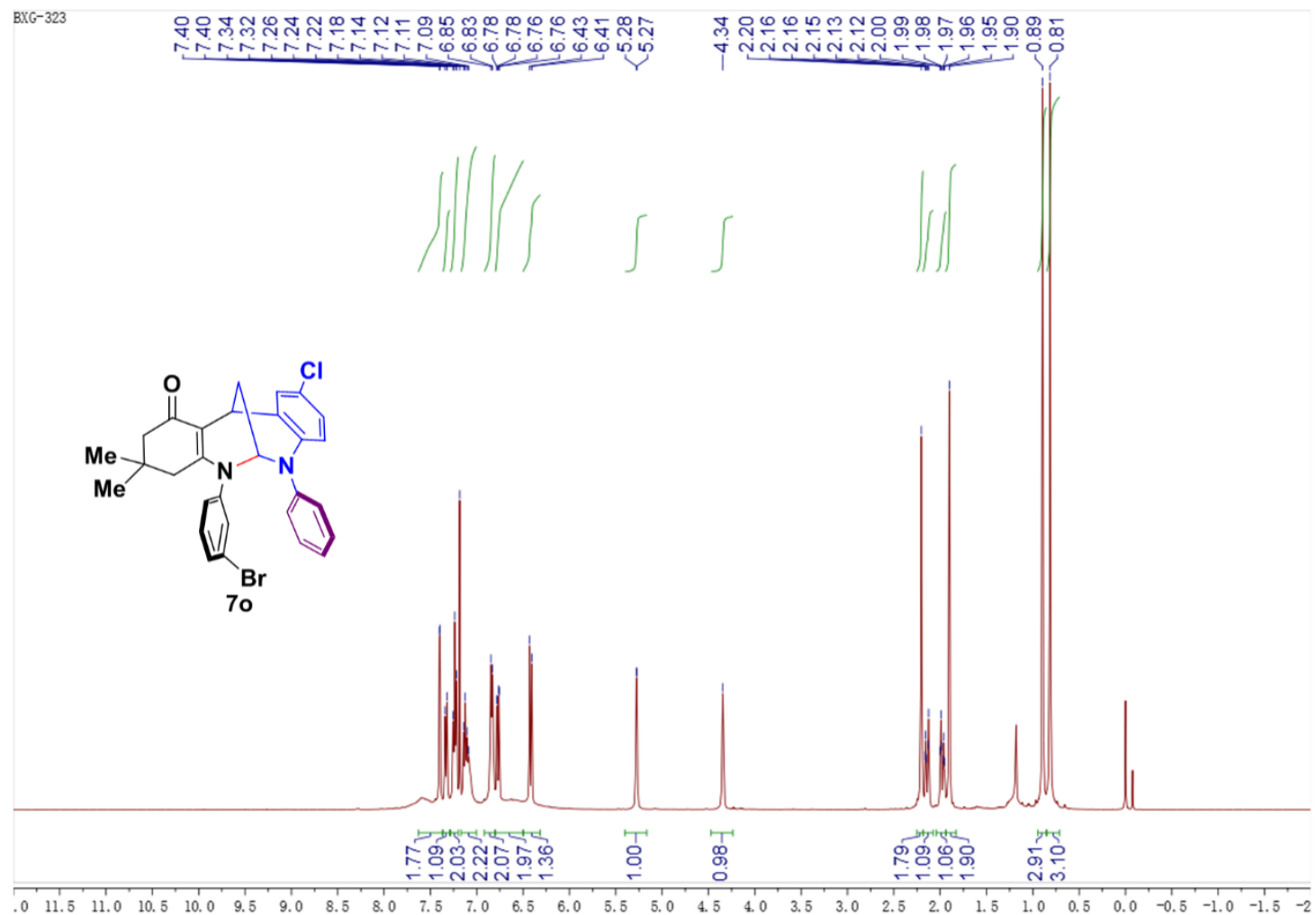

${ }^{13} \mathrm{C}$ NMR spectrum of $7 \mathbf{o}\left(100 \mathrm{MHz}, \mathrm{CDCl}_{3}\right)$ 


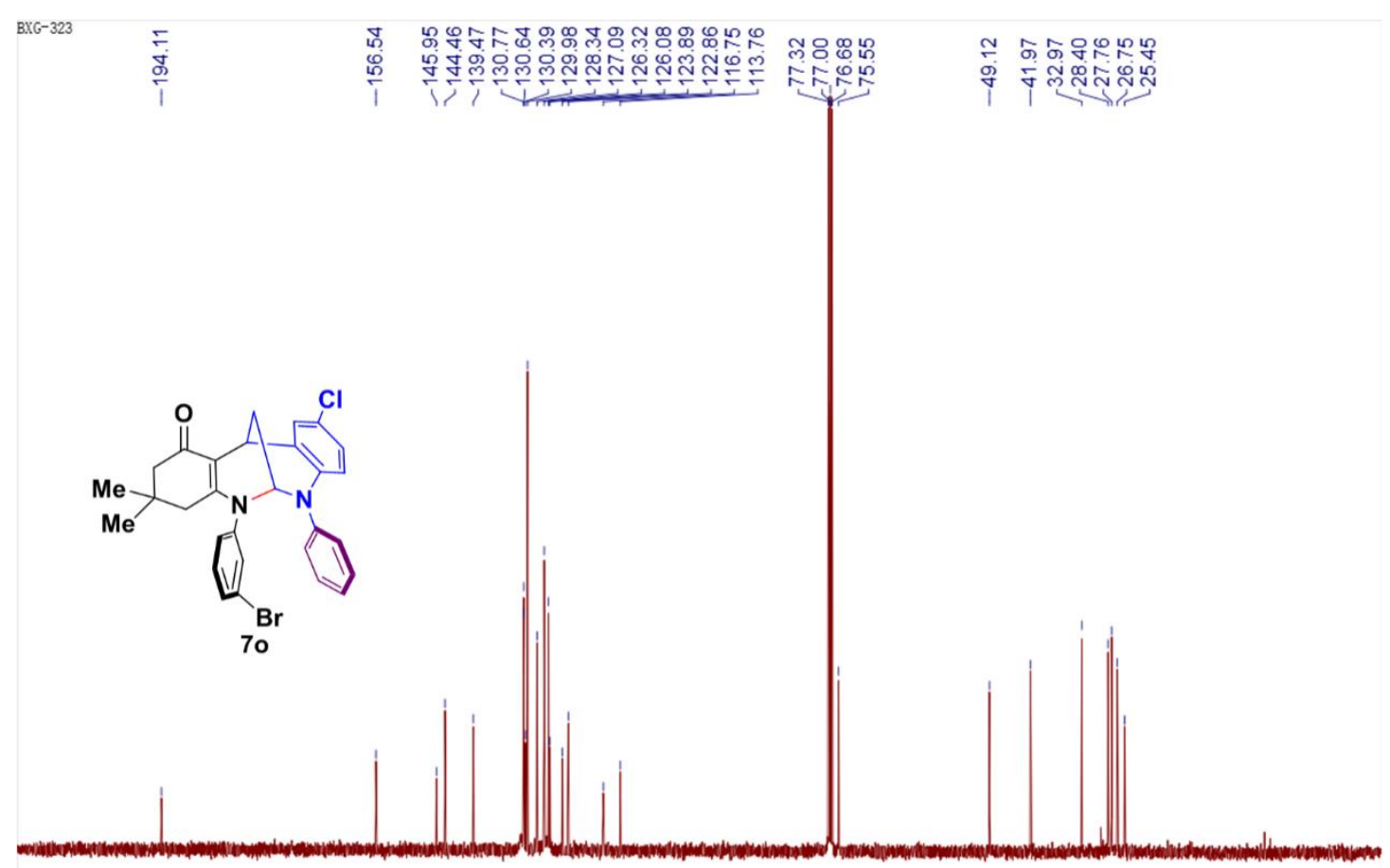

${ }^{1} \mathrm{H}$ NMR spectrum of $9 \mathbf{a}\left(400 \mathrm{MHz}, \mathrm{CDCl}_{3}\right)$

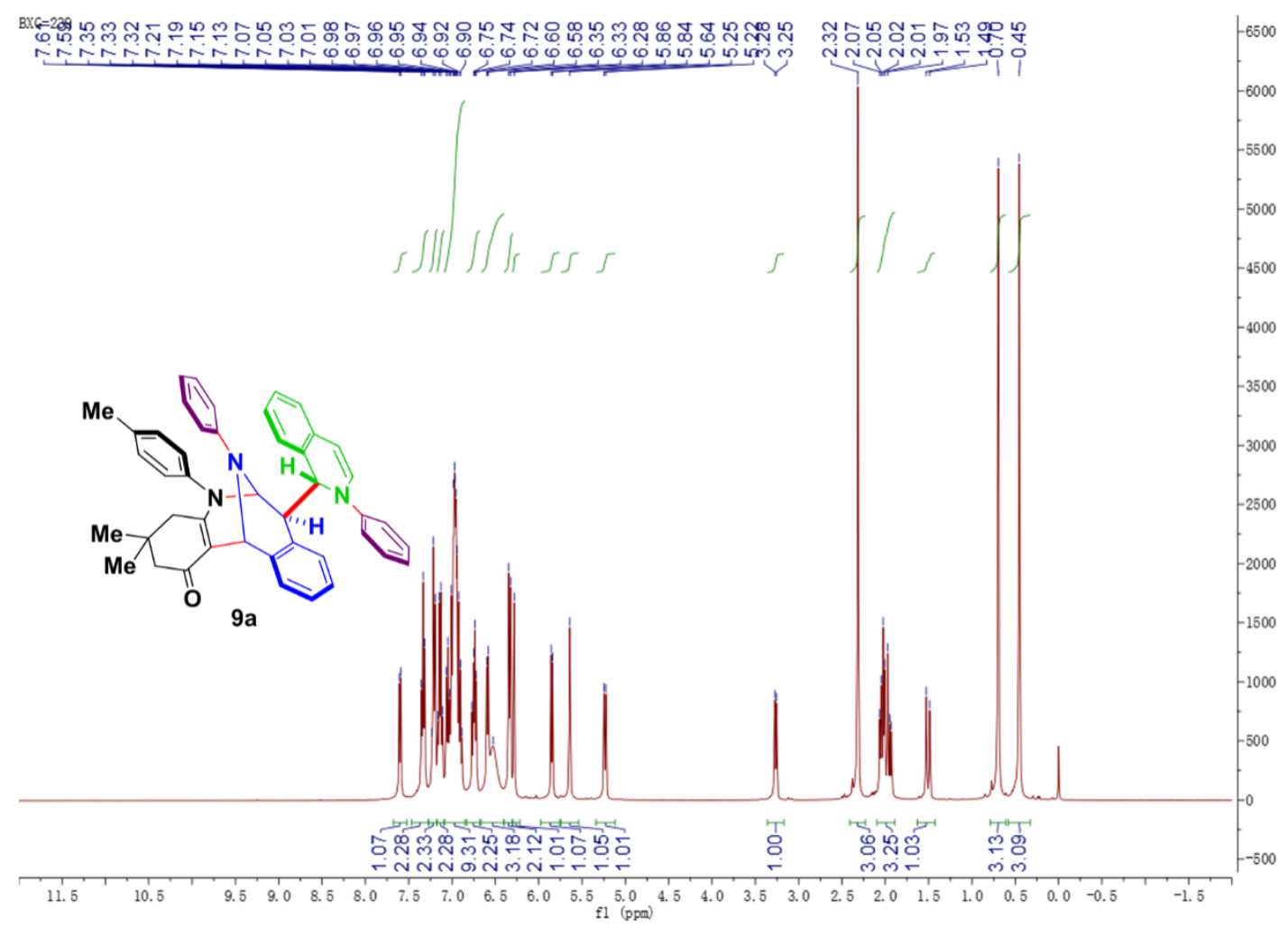

${ }^{13} \mathrm{C}$ NMR spectrum of $9 \mathbf{a}\left(100 \mathrm{MHz}, \mathrm{CDCl}_{3}\right)$ 


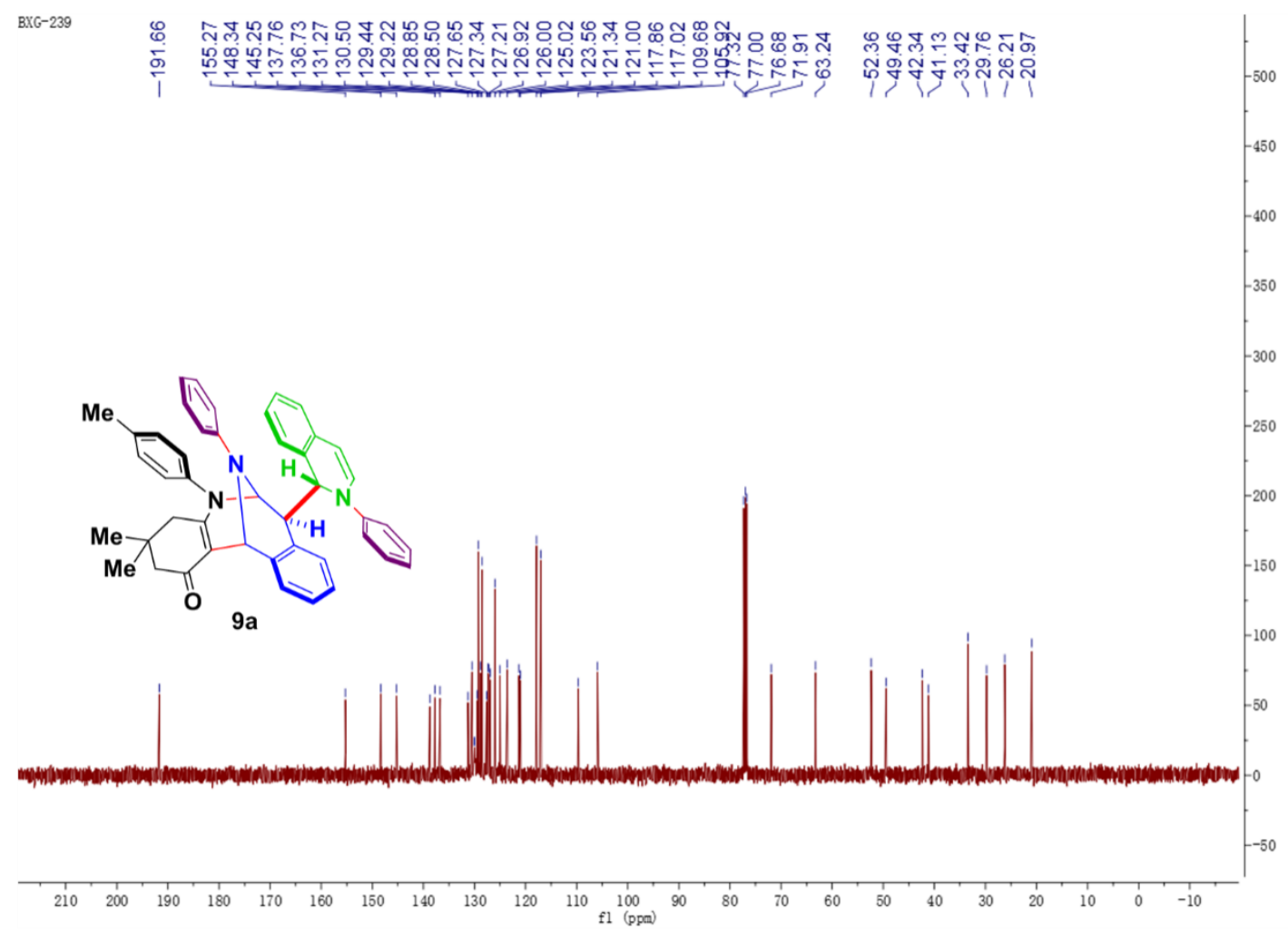

${ }^{1} \mathrm{H}$ NMR spectrum of $\mathbf{9 b}\left(400 \mathrm{MHz}, \mathrm{CDCl}_{3}\right)$

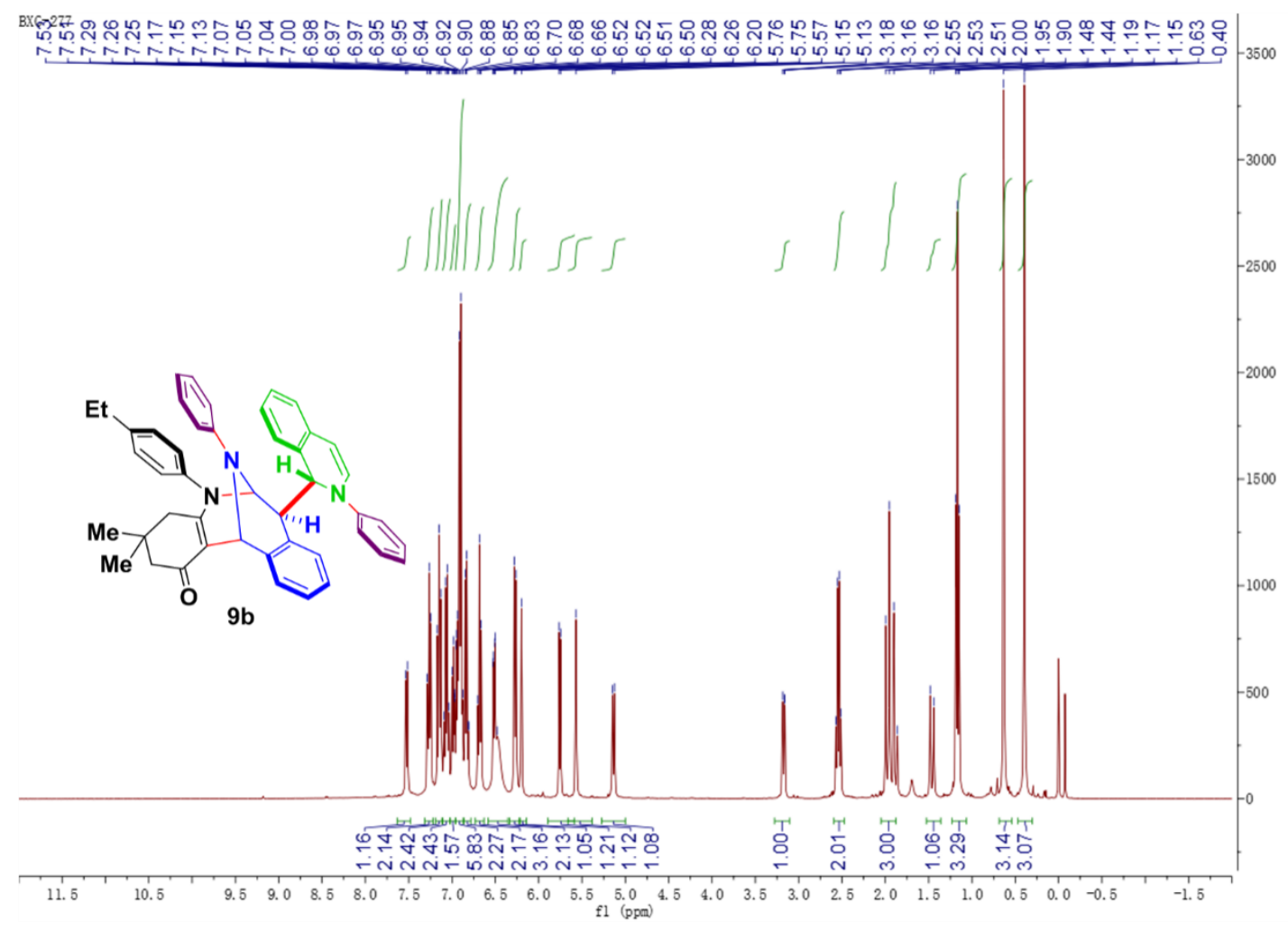

${ }^{13} \mathrm{C}$ NMR spectrum of $\mathbf{9 b}\left(100 \mathrm{MHz}, \mathrm{CDCl}_{3}\right)$ 


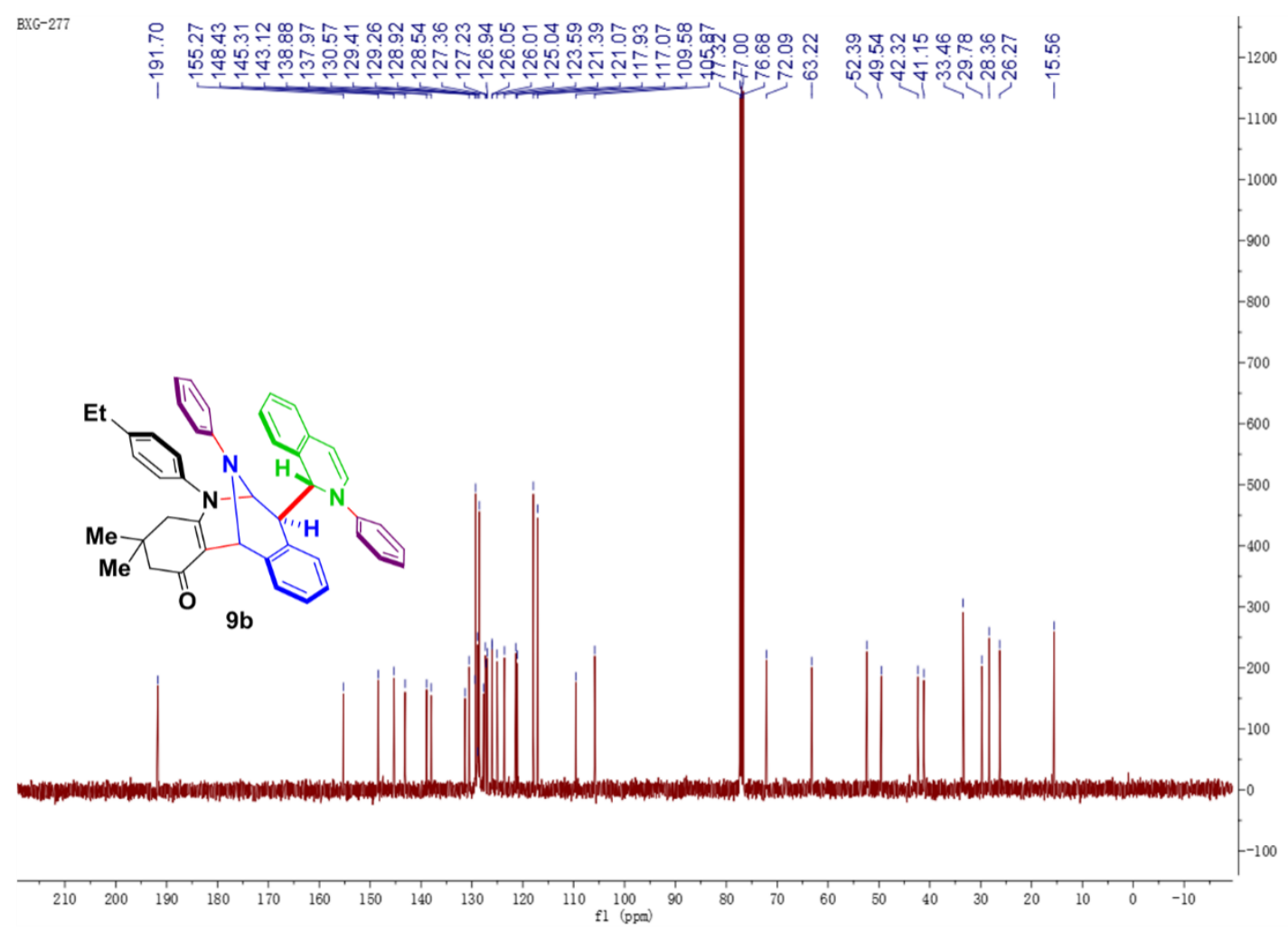

${ }^{1} \mathrm{H}$ NMR spectrum of $9 \mathrm{c}\left(400 \mathrm{MHz}, \mathrm{CDCl}_{3}\right)$

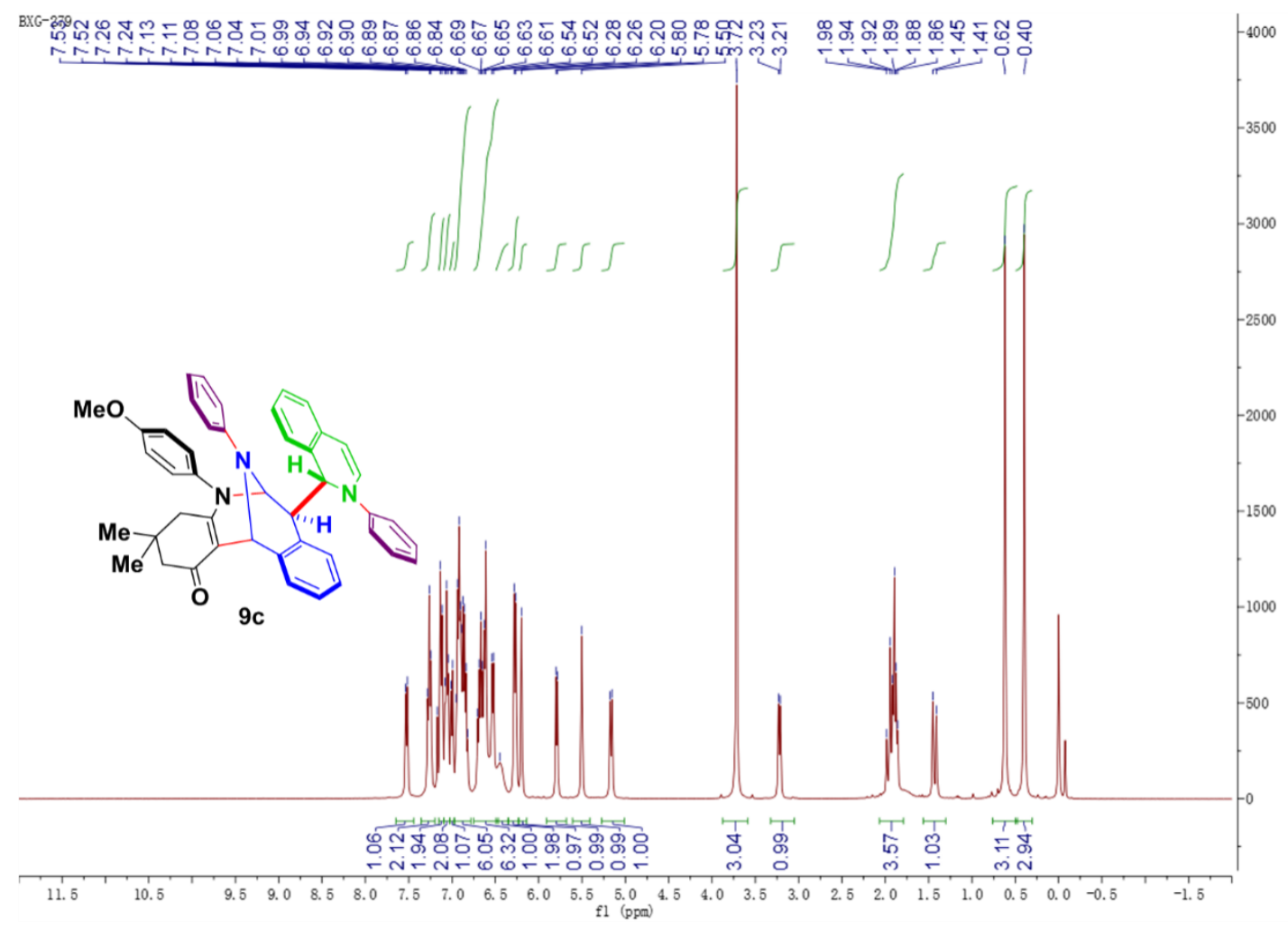

${ }^{13} \mathrm{C}$ NMR spectrum of $9 c\left(100 \mathrm{MHz}, \mathrm{CDCl}_{3}\right)$ 


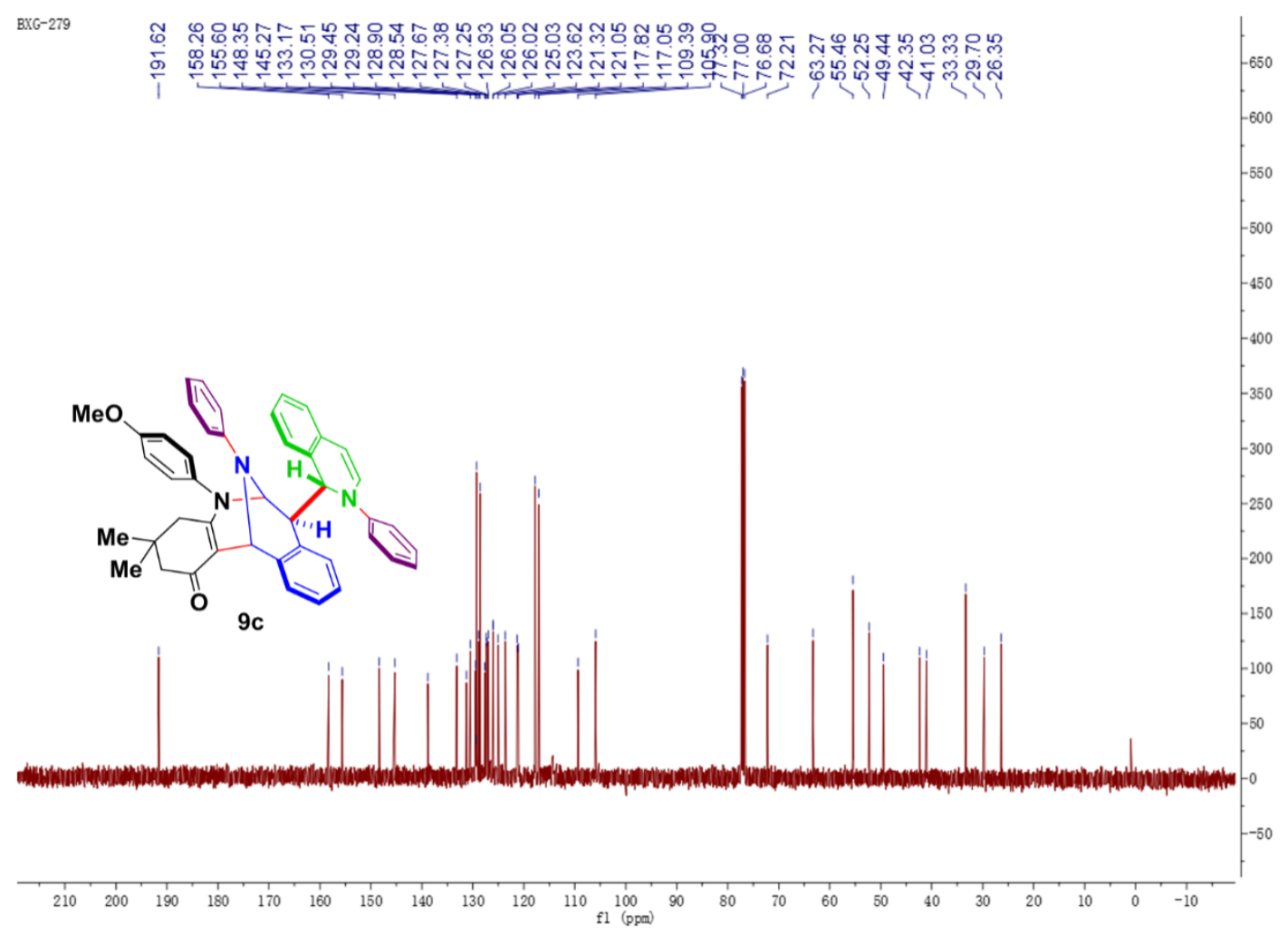

${ }^{1} \mathrm{H}$ NMR spectrum of $9 d\left(400 \mathrm{MHz}, \mathrm{CDCl}_{3}\right)$

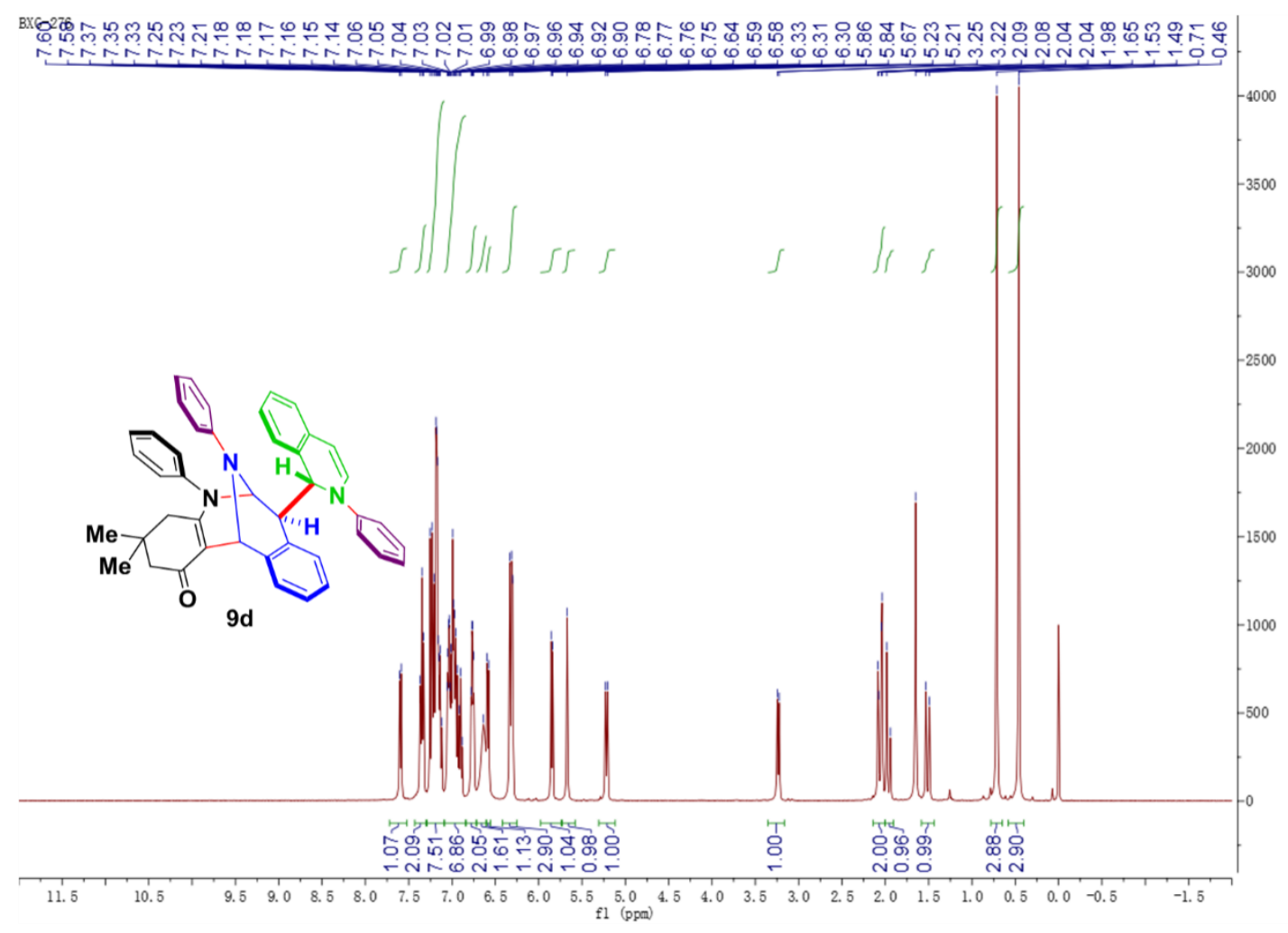

${ }^{13} \mathrm{C}$ NMR spectrum of 9d $\left(100 \mathrm{MHz}, \mathrm{CDCl}_{3}\right)$ 


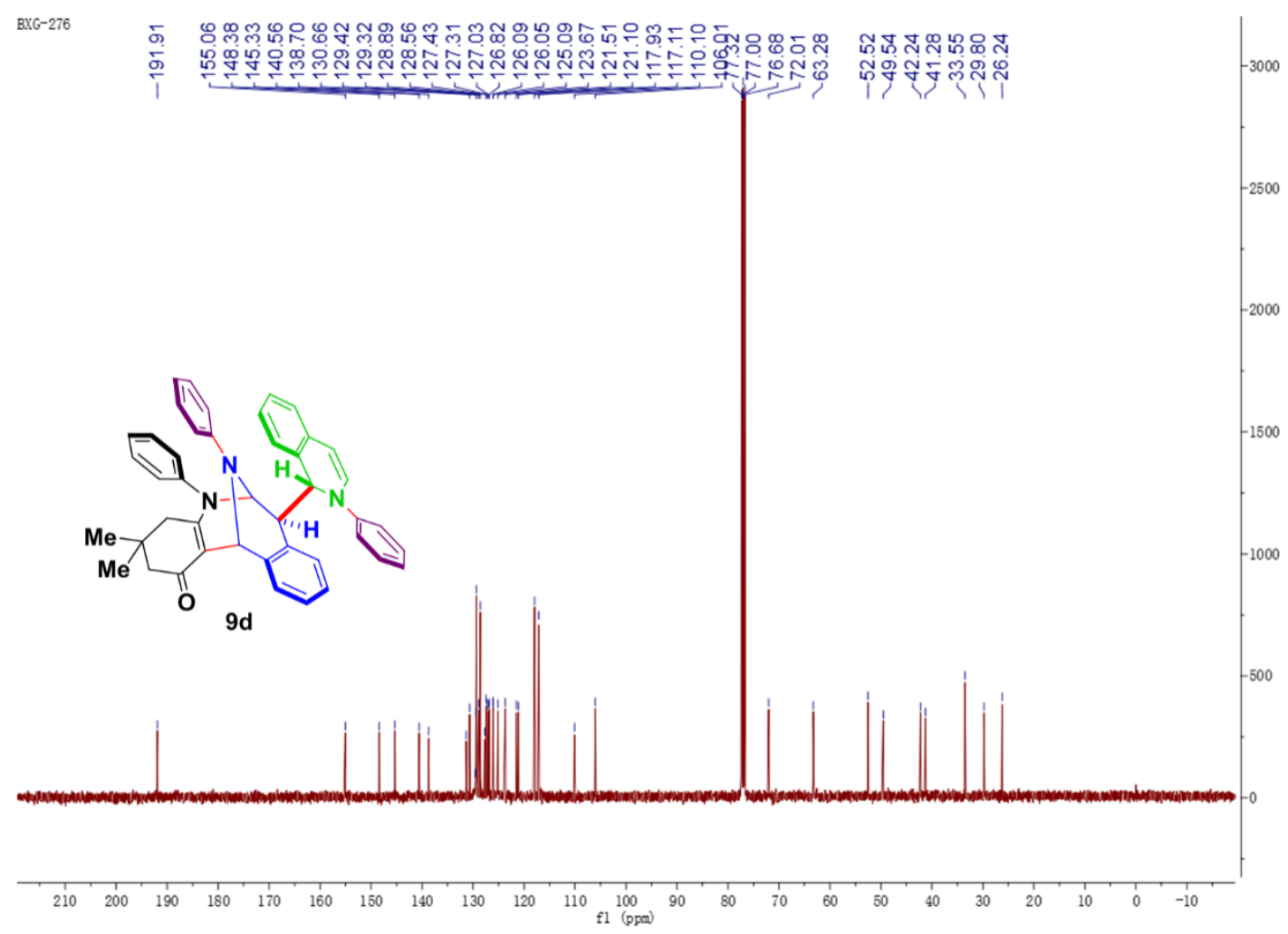

${ }^{1} \mathrm{H}$ NMR spectrum of $9 \mathbf{e}\left(400 \mathrm{MHz}, \mathrm{CDCl}_{3}\right)$

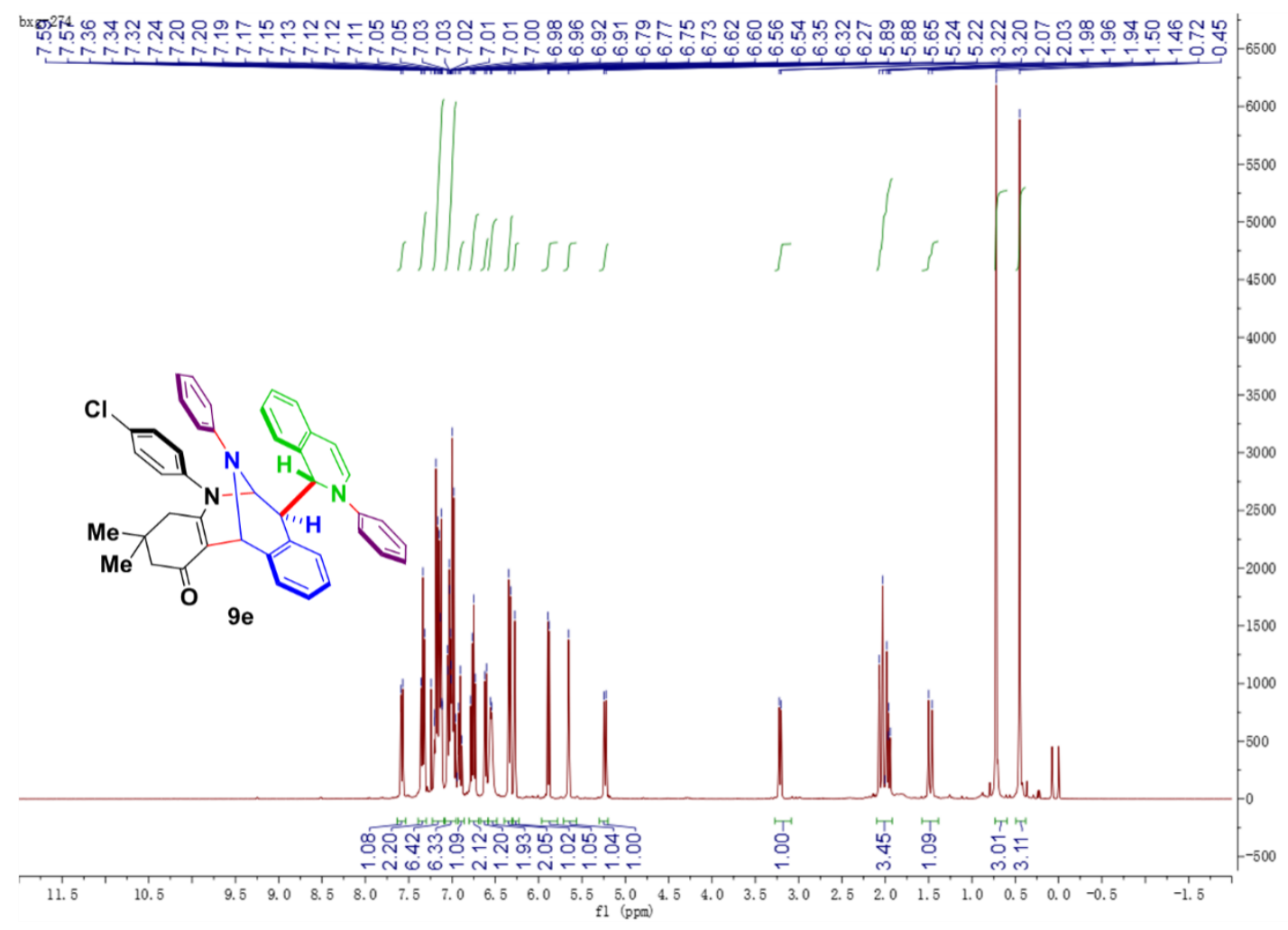

${ }^{13} \mathrm{C}$ NMR spectrum of $9 e\left(100 \mathrm{MHz}, \mathrm{CDCl}_{3}\right)$ 


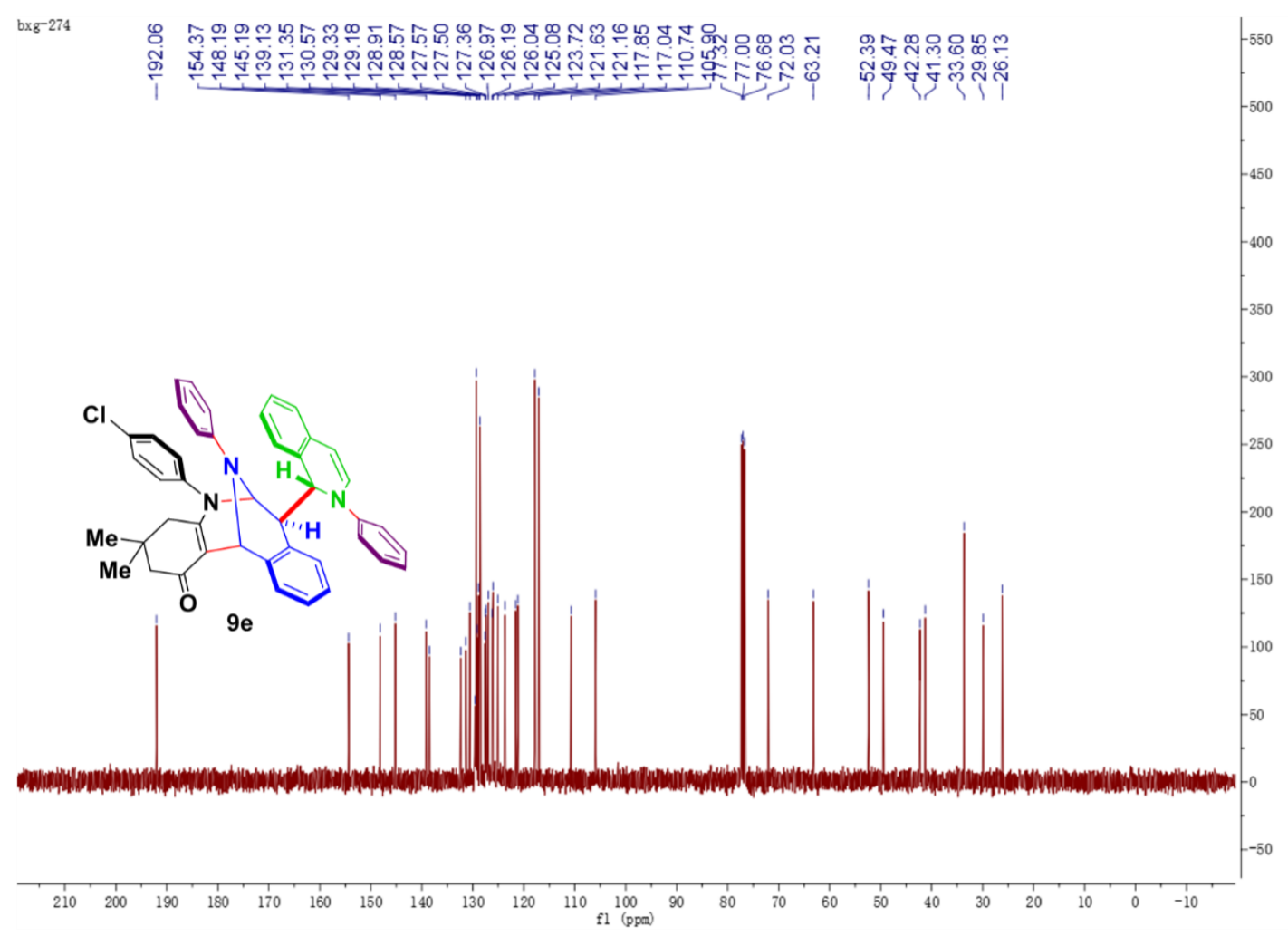

${ }^{1} \mathrm{H}$ NMR spectrum of $9 f\left(400 \mathrm{MHz}, \mathrm{CDCl}_{3}\right)$

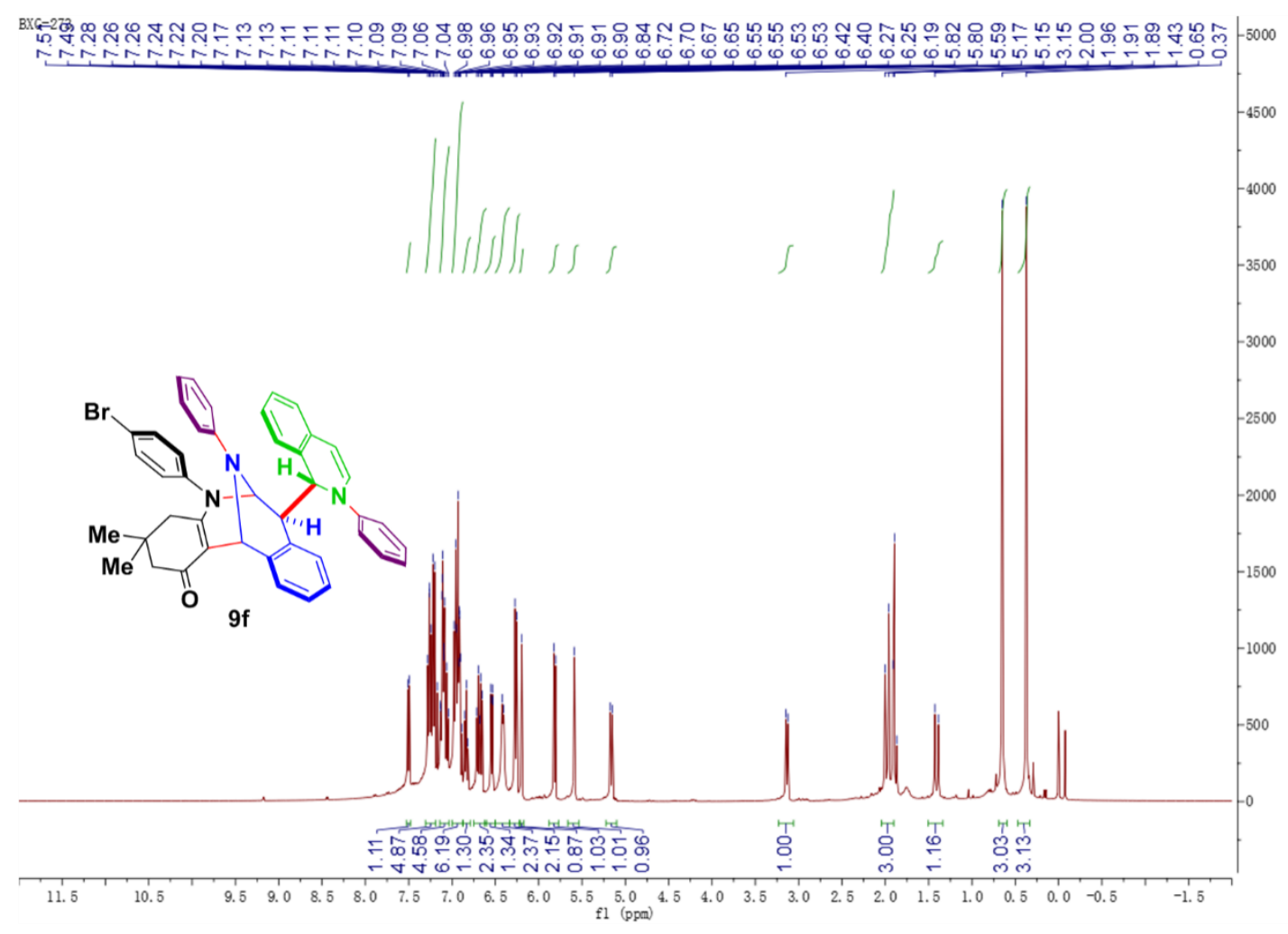

${ }^{13} \mathrm{C}$ NMR spectrum of $9 f\left(100 \mathrm{MHz}, \mathrm{CDCl}_{3}\right)$ 


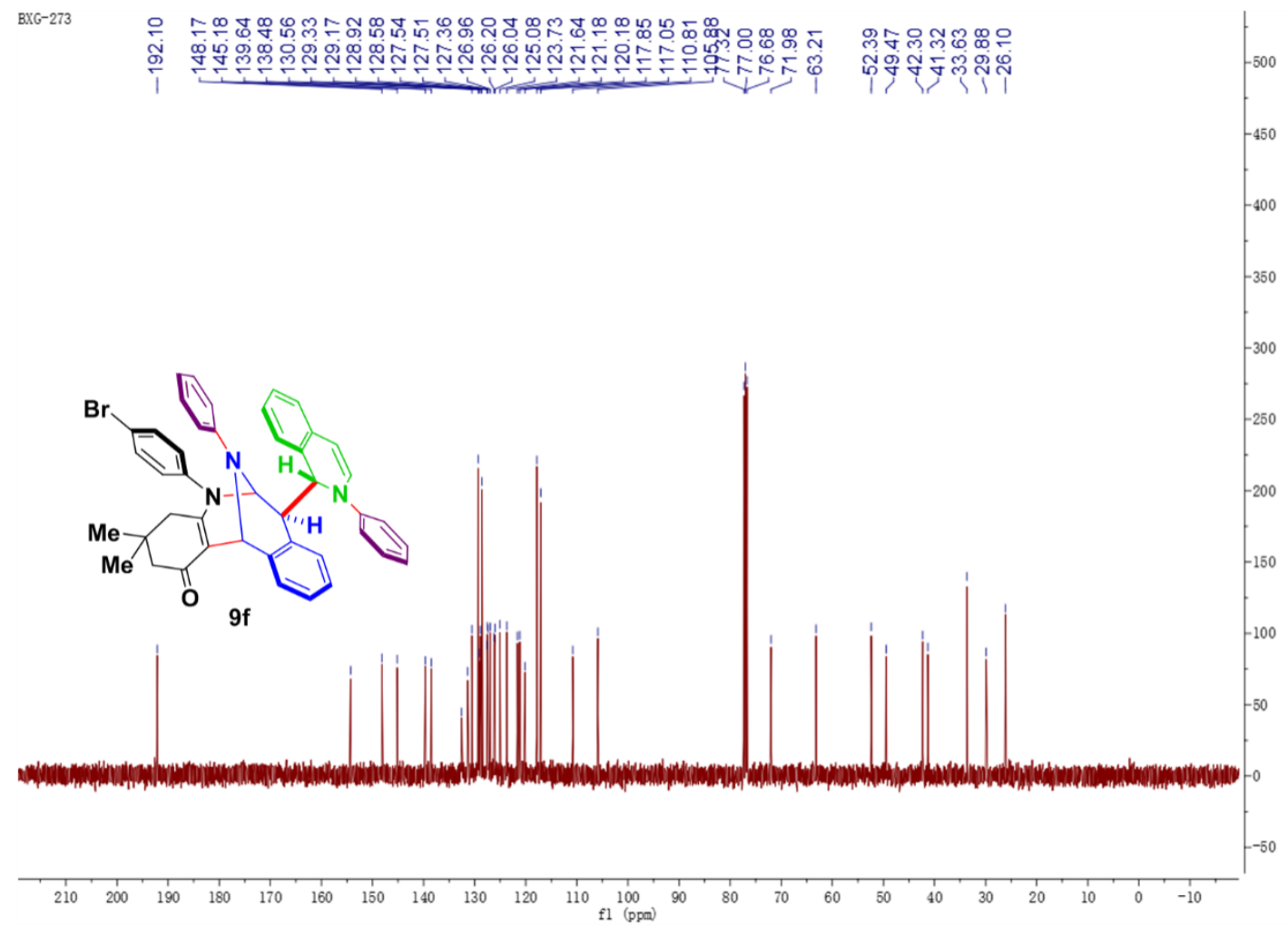

${ }^{1} \mathrm{H}$ NMR spectrum of $9 \mathbf{g}\left(400 \mathrm{MHz}, \mathrm{CDCl}_{3}\right)$

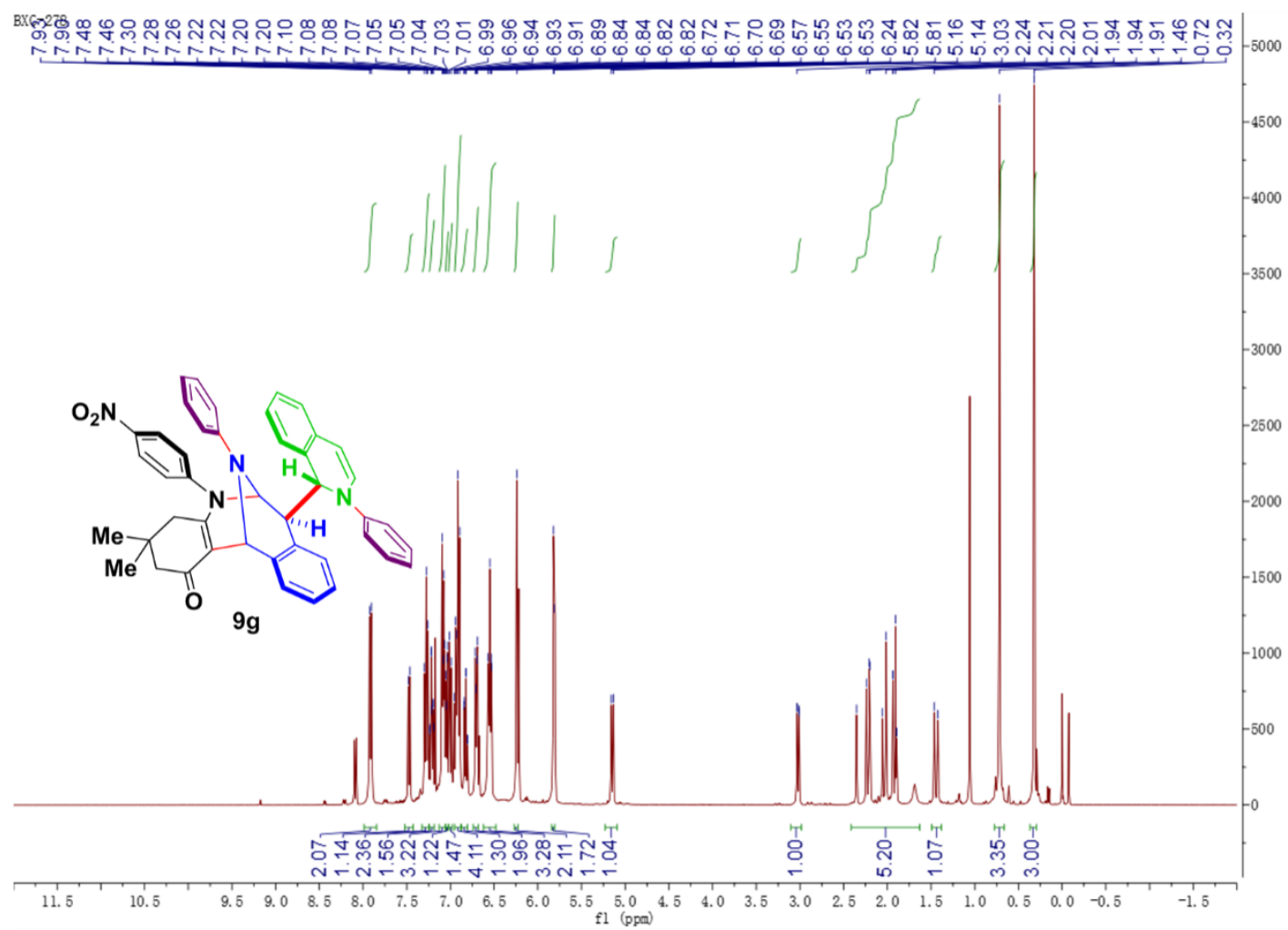

${ }^{13} \mathrm{C}$ NMR spectrum of $9 \mathrm{~g}\left(100 \mathrm{MHz}, \mathrm{CDCl}_{3}\right)$ 


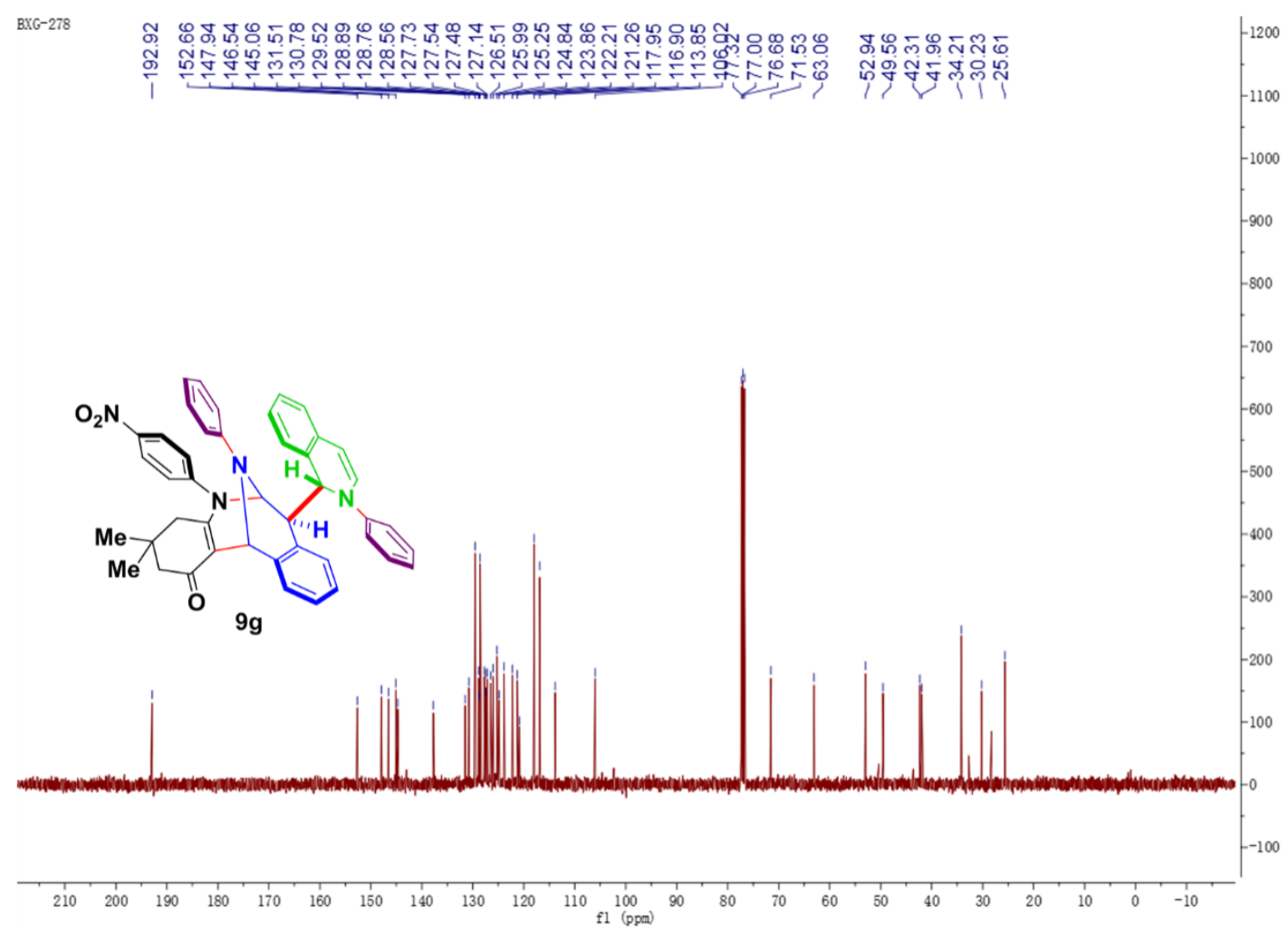

${ }^{1} \mathrm{H}$ NMR spectrum of $\mathbf{9 h}\left(400 \mathrm{MHz}, \mathrm{CDCl}_{3}\right)$

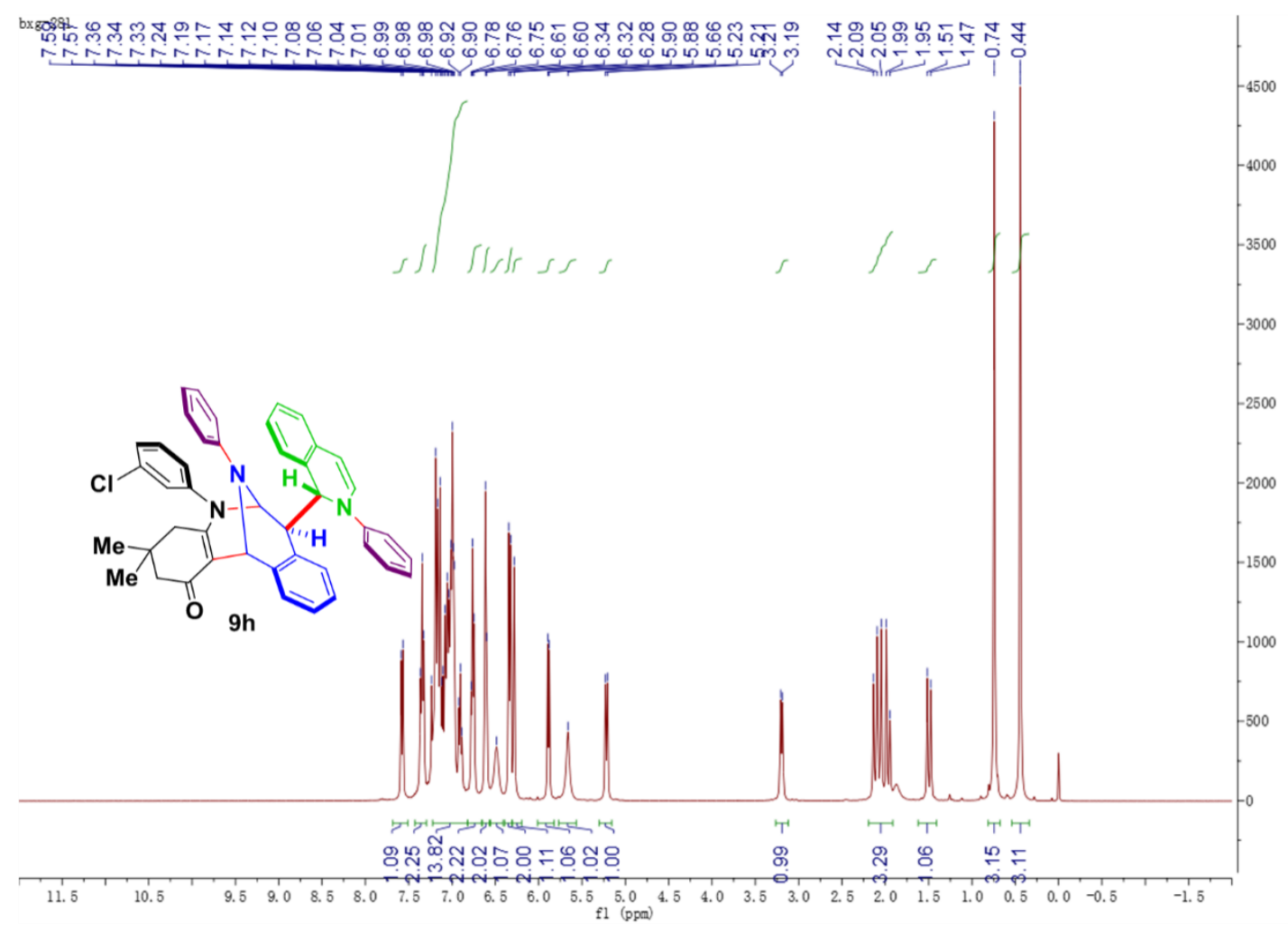

${ }^{13} \mathrm{C}$ NMR spectrum of $\mathbf{9 h}\left(100 \mathrm{MHz}, \mathrm{CDCl}_{3}\right)$ 


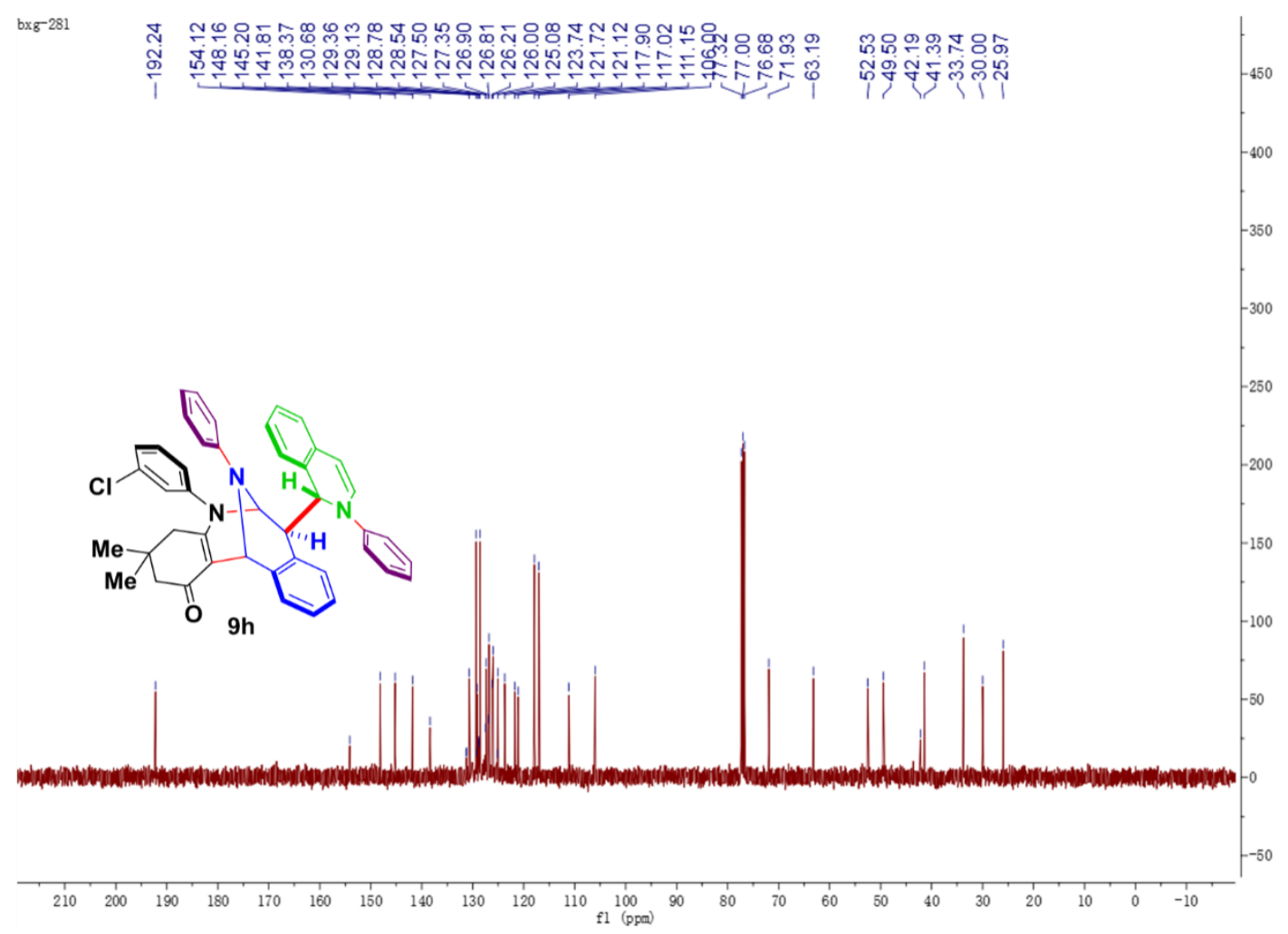

${ }^{1} \mathrm{H}$ NMR spectrum of $\mathbf{9 i}\left(400 \mathrm{MHz}, \mathrm{CDCl}_{3}\right)$

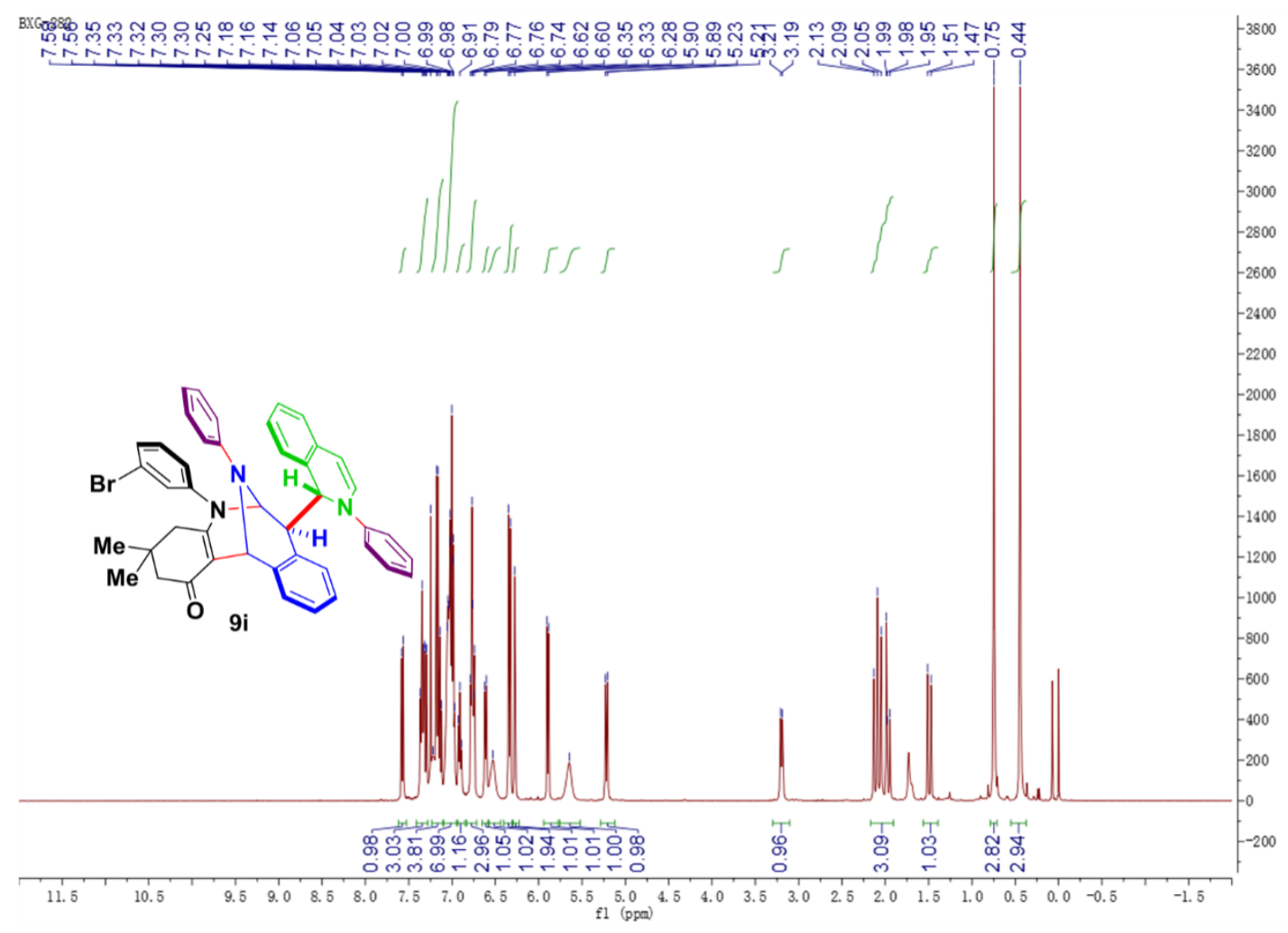

${ }^{13} \mathrm{C}$ NMR spectrum of $\mathbf{9 i}\left(100 \mathrm{MHz}, \mathrm{CDCl}_{3}\right)$ 


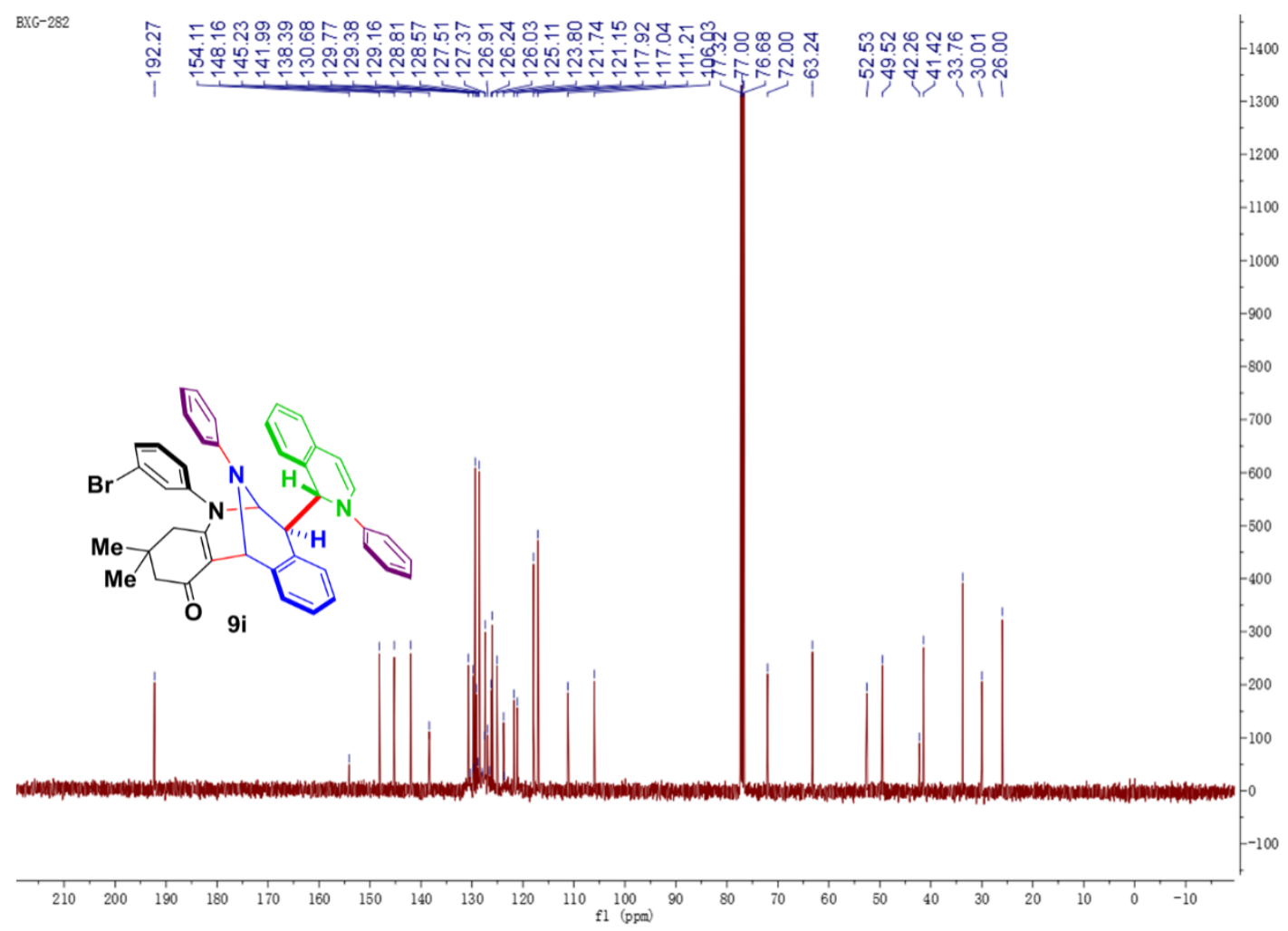

${ }^{1} \mathrm{H}$ NMR spectrum of $\mathbf{9 j}\left(400 \mathrm{MHz}, \mathrm{CDCl}_{3}\right)$

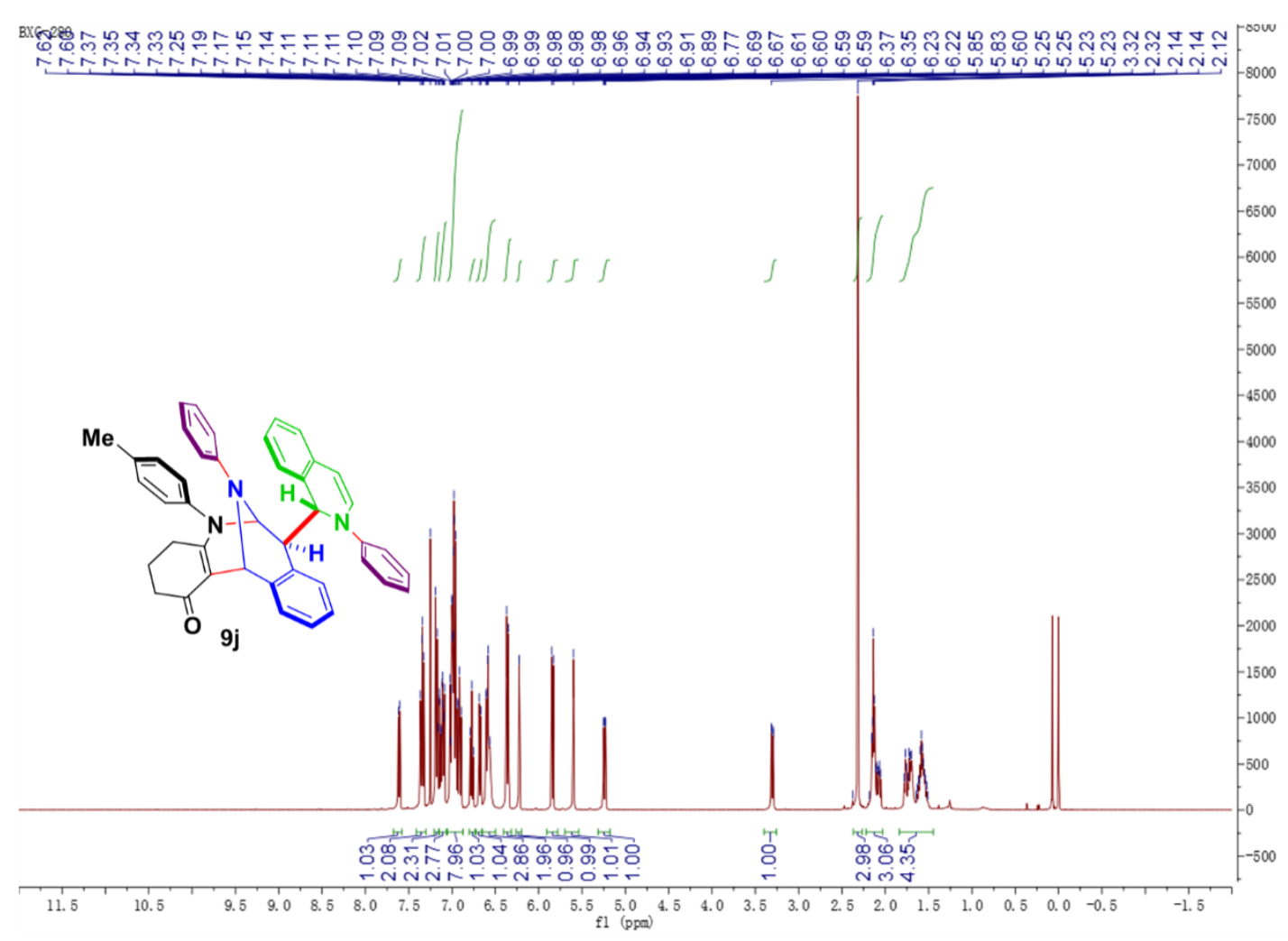

${ }^{13} \mathrm{C}$ NMR spectrum of $\mathbf{9 j}\left(100 \mathrm{MHz}, \mathrm{CDCl}_{3}\right)$ 


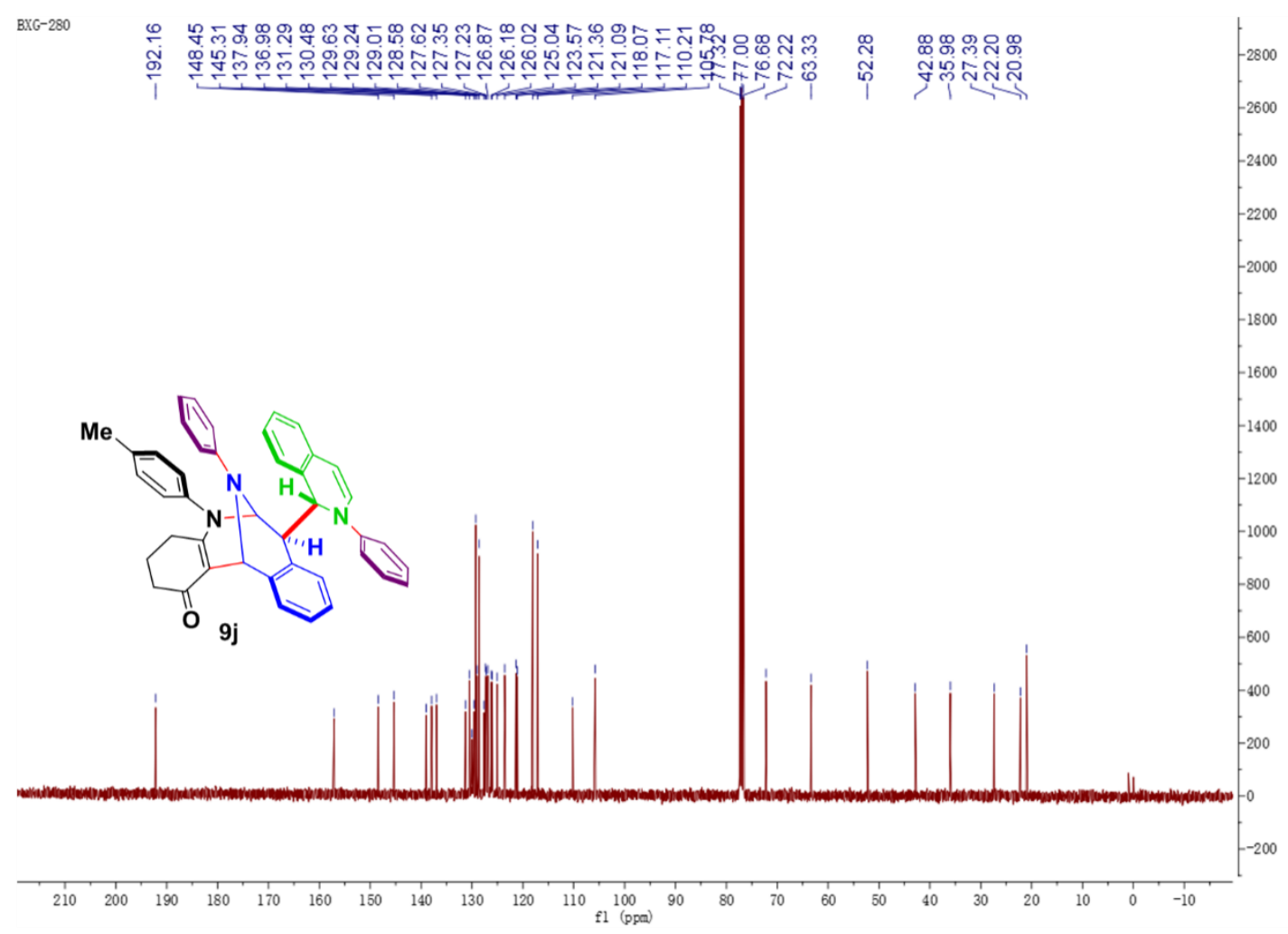

${ }^{1} \mathrm{H}$ NMR spectrum of $9 \mathbf{k}\left(400 \mathrm{MHz}, \mathrm{CDCl}_{3}\right)$

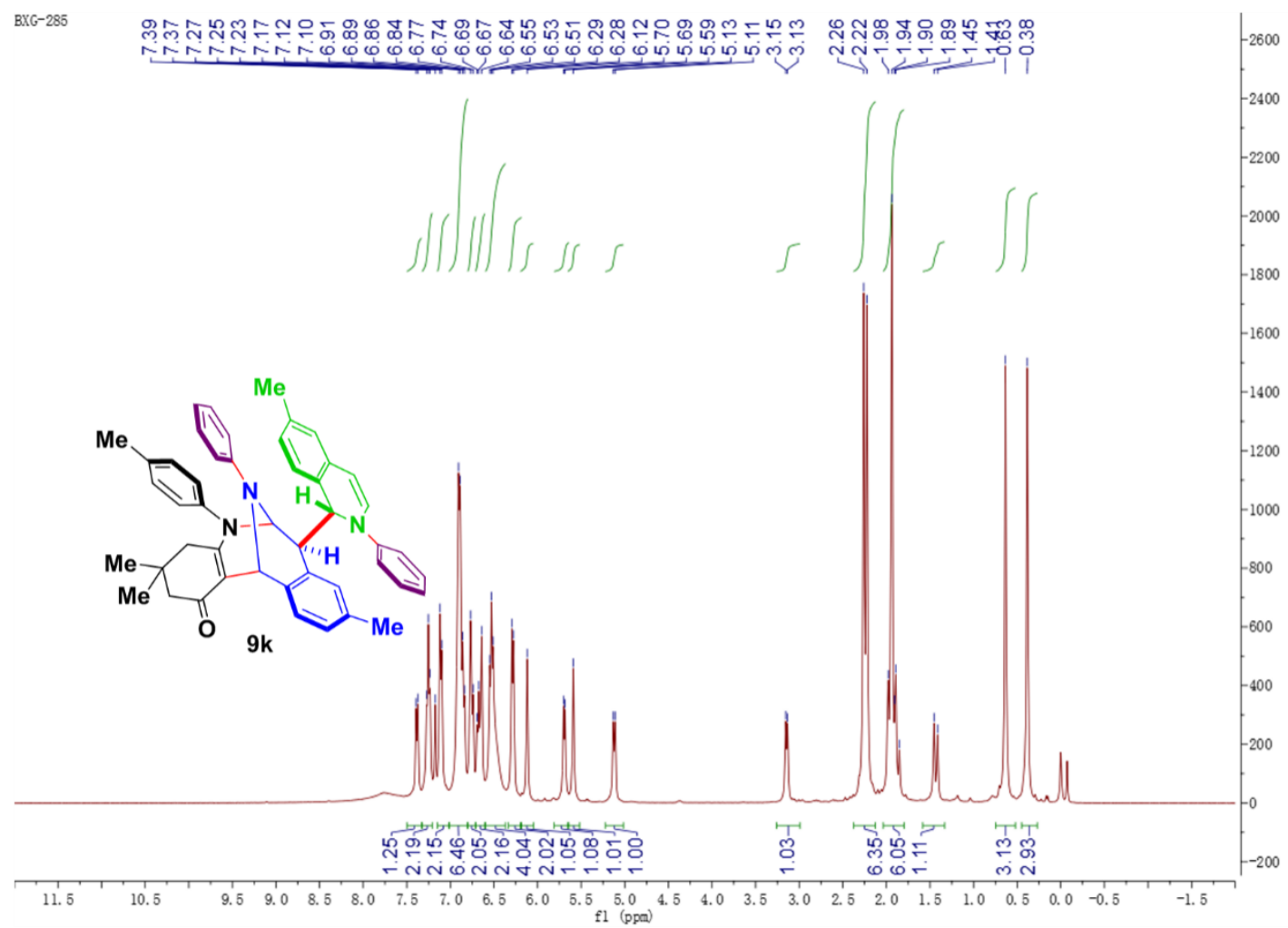

${ }^{13} \mathrm{C}$ NMR spectrum of $9 \mathbf{k}\left(100 \mathrm{MHz}, \mathrm{CDCl}_{3}\right)$ 


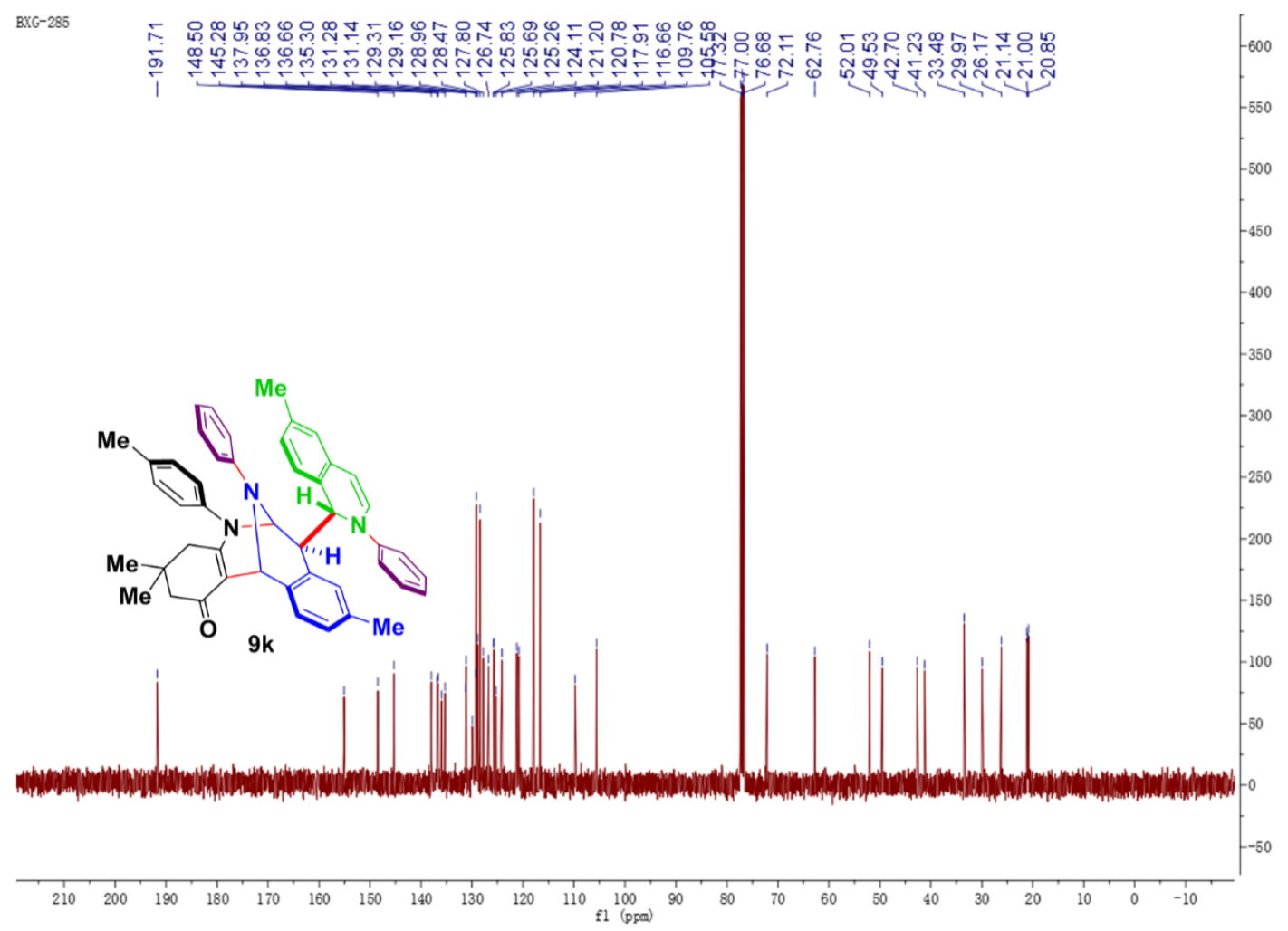

${ }^{1} \mathrm{H}$ NMR spectrum of $91\left(400 \mathrm{MHz}, \mathrm{CDCl}_{3}\right)$

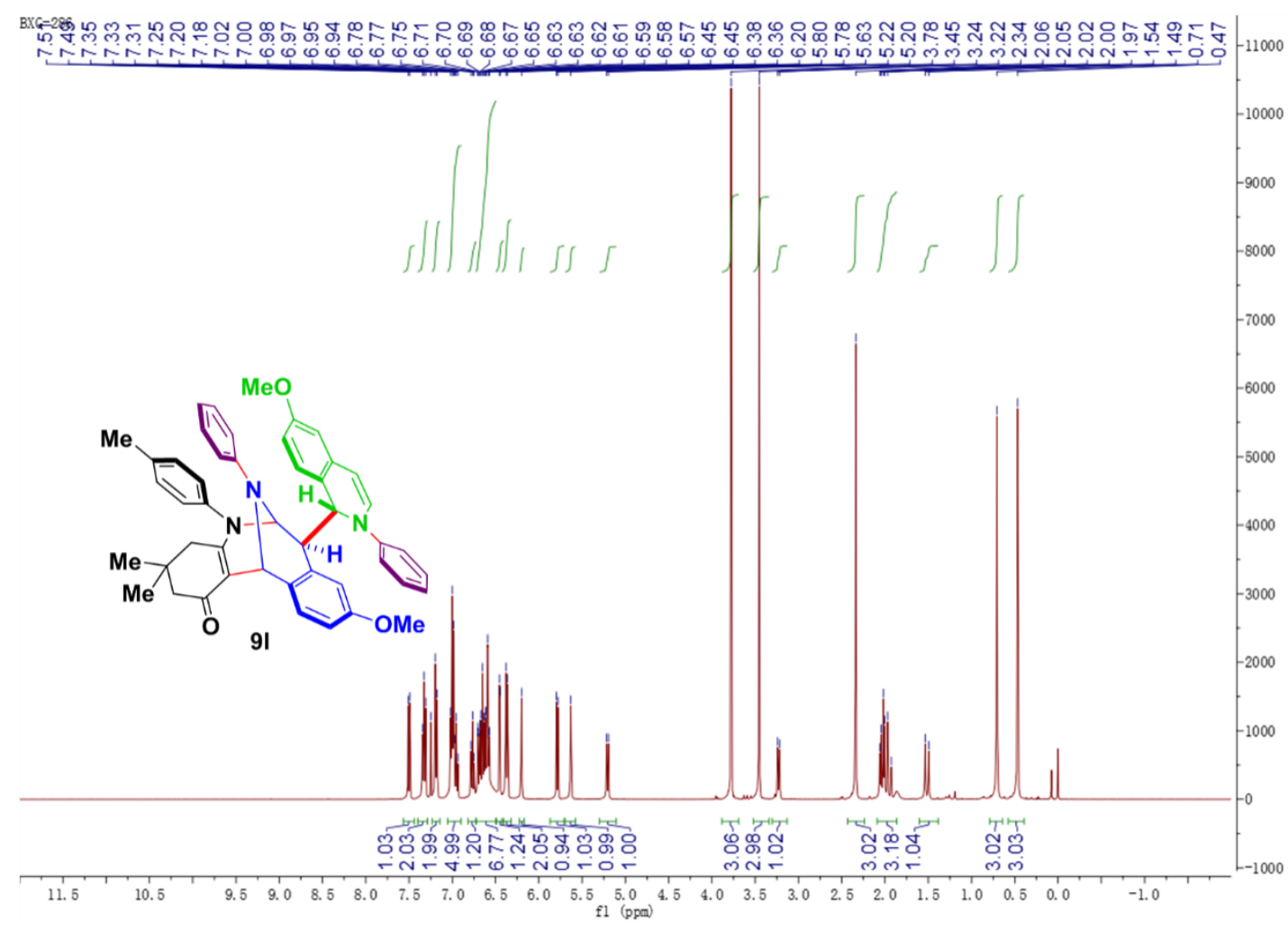

${ }^{13} \mathrm{C}$ NMR spectrum of $91\left(100 \mathrm{MHz}, \mathrm{CDCl}_{3}\right)$ 


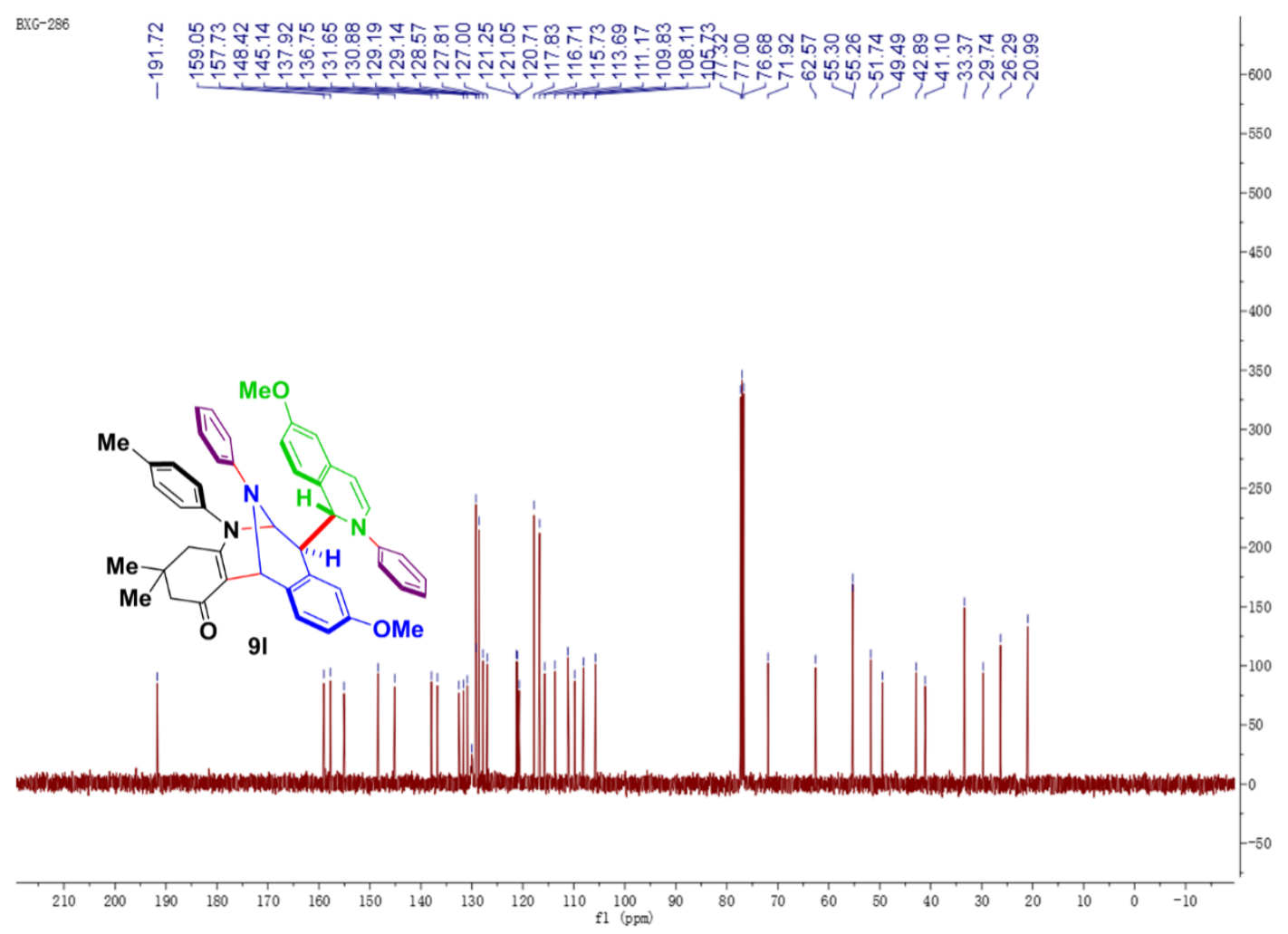

${ }^{1} \mathrm{H}$ NMR spectrum of $9 m\left(400 \mathrm{MHz}, \mathrm{CDCl}_{3}\right)$

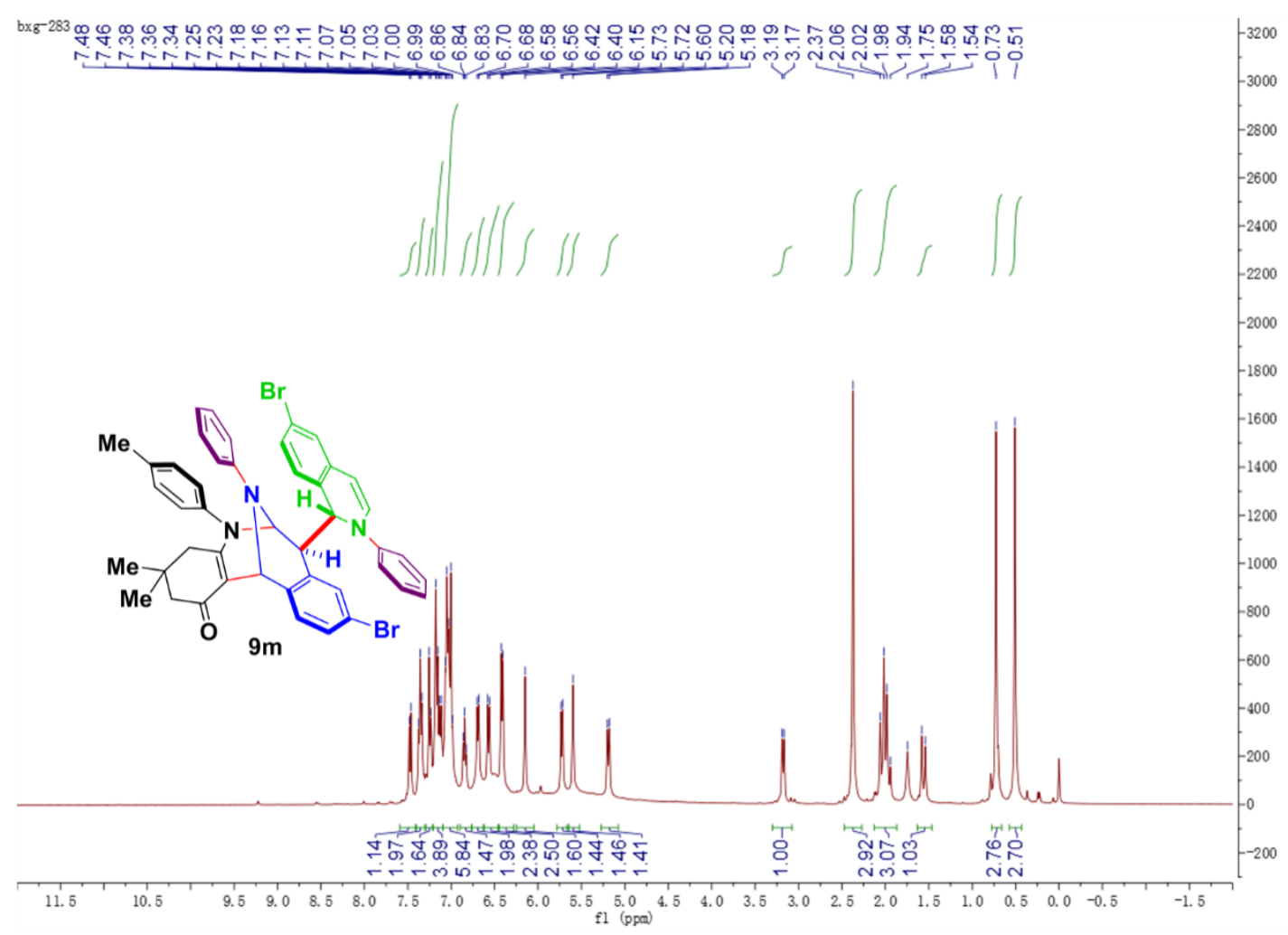

${ }^{13} \mathrm{C}$ NMR spectrum of $9 \mathrm{~m}\left(100 \mathrm{MHz}, \mathrm{CDCl}_{3}\right)$ 


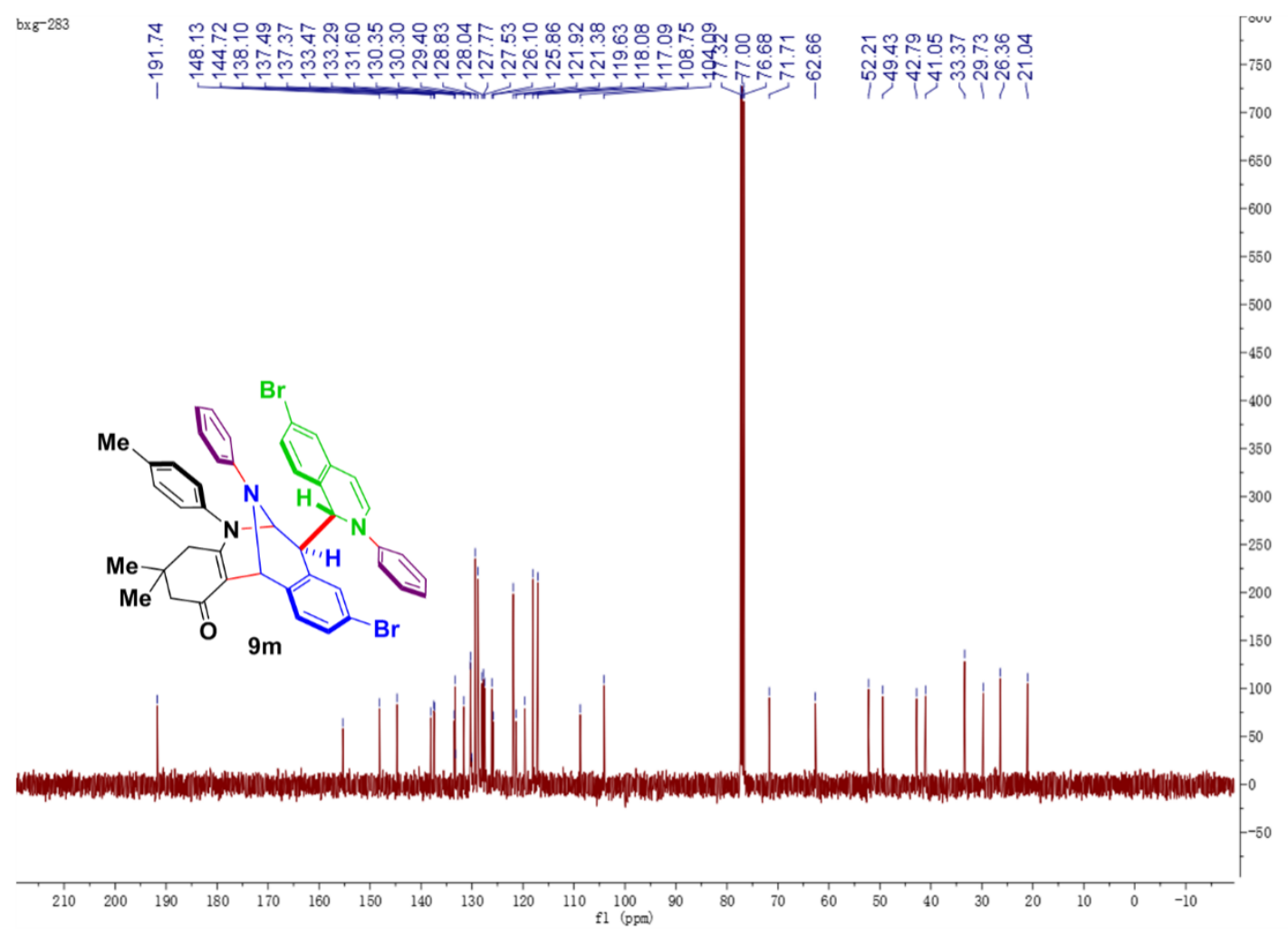

${ }^{1} \mathrm{H}$ NMR spectrum of $9 \mathbf{n}\left(400 \mathrm{MHz}, \mathrm{CDCl}_{3}\right)$

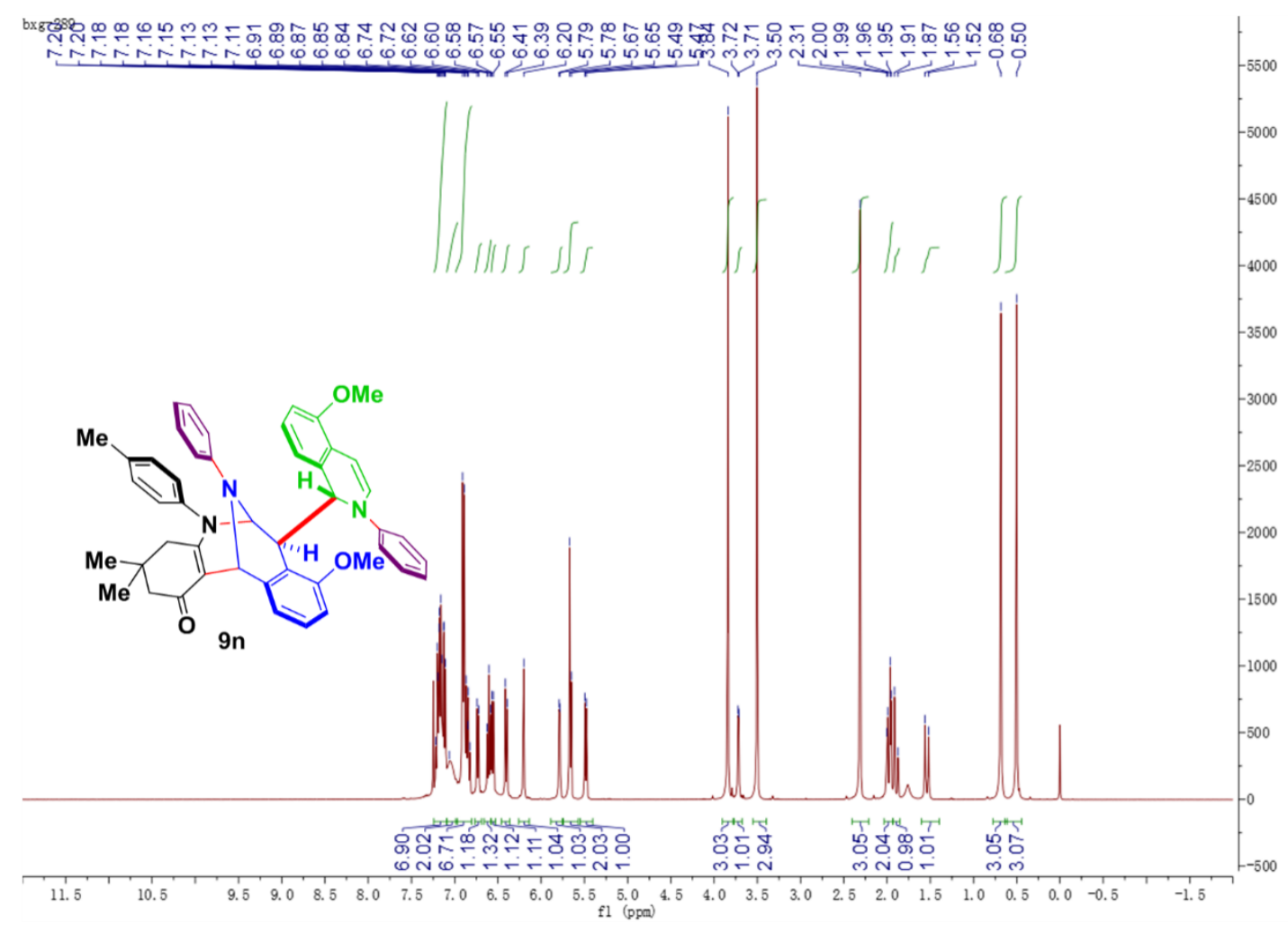

${ }^{13} \mathrm{C}$ NMR spectrum of $9 \mathbf{n}\left(100 \mathrm{MHz}, \mathrm{CDCl}_{3}\right)$ 


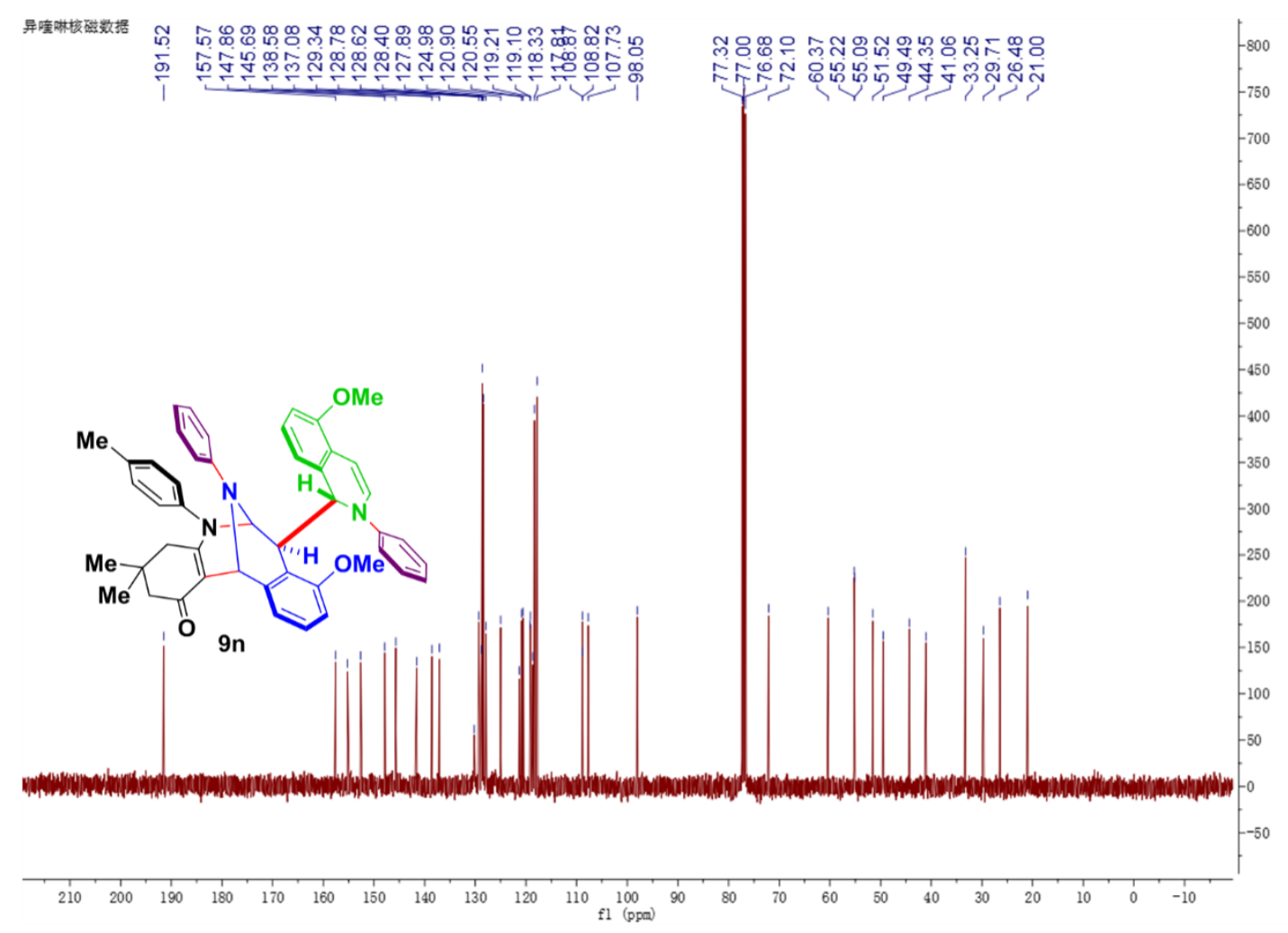

${ }^{1} \mathrm{H}$ NMR spectrum of $90\left(400 \mathrm{MHz}, \mathrm{CDCl}_{3}\right)$

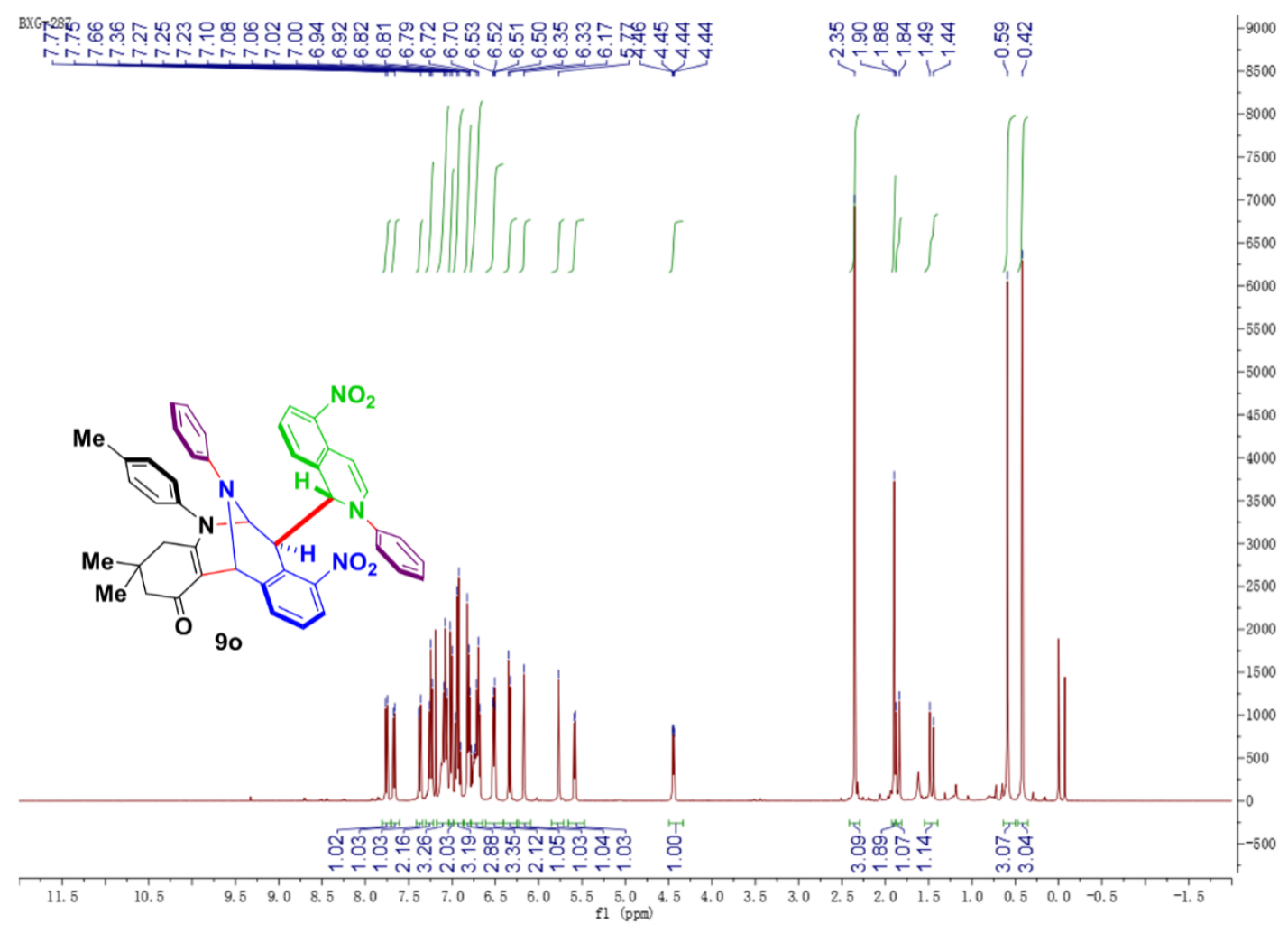

${ }^{13} \mathrm{C}$ NMR spectrum of $90\left(100 \mathrm{MHz}, \mathrm{CDCl}_{3}\right)$ 


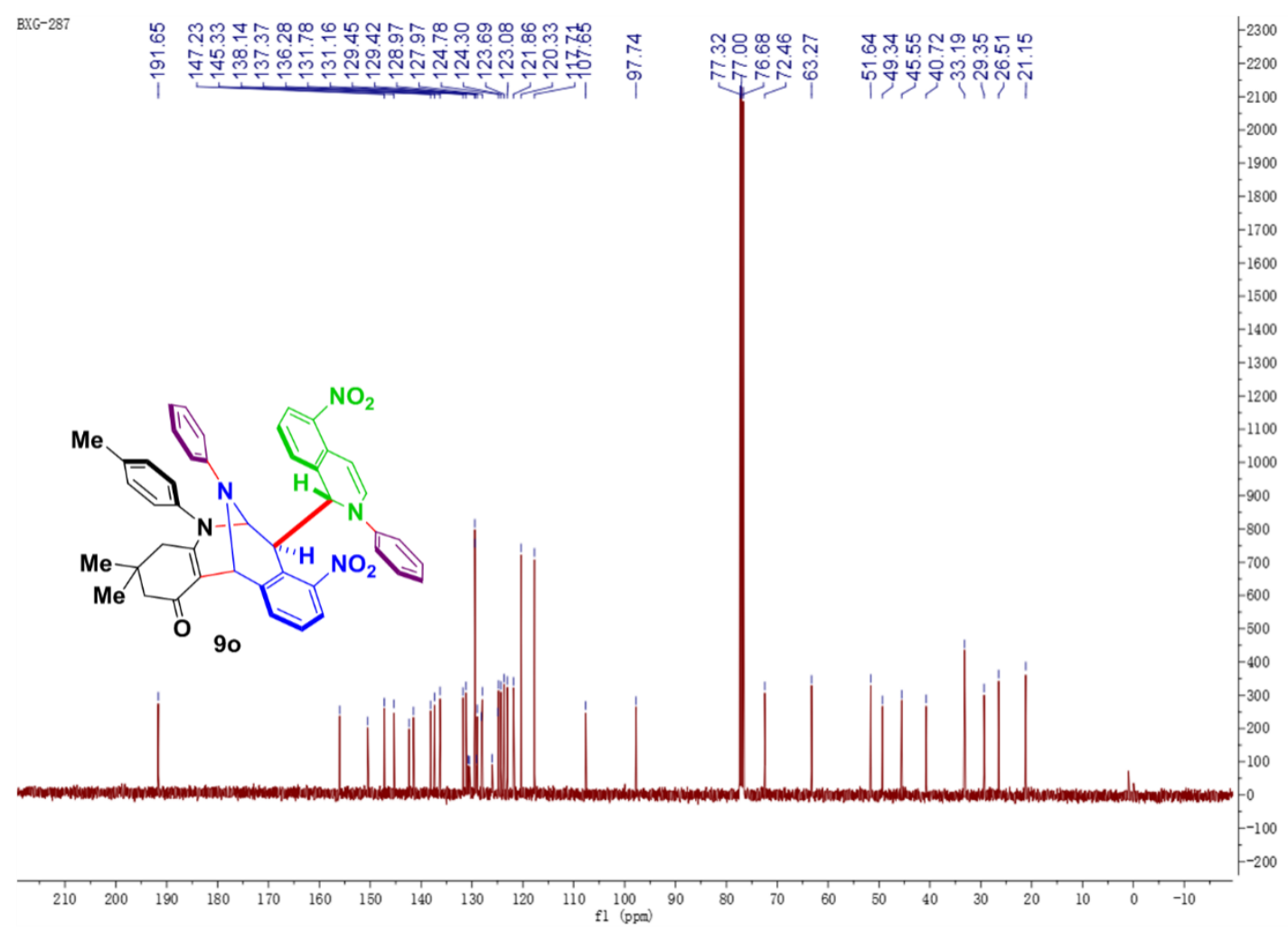

${ }^{1} \mathrm{H}$ NMR spectrum of $9 \mathbf{p}\left(400 \mathrm{MHz}, \mathrm{CDCl}_{3}\right)$

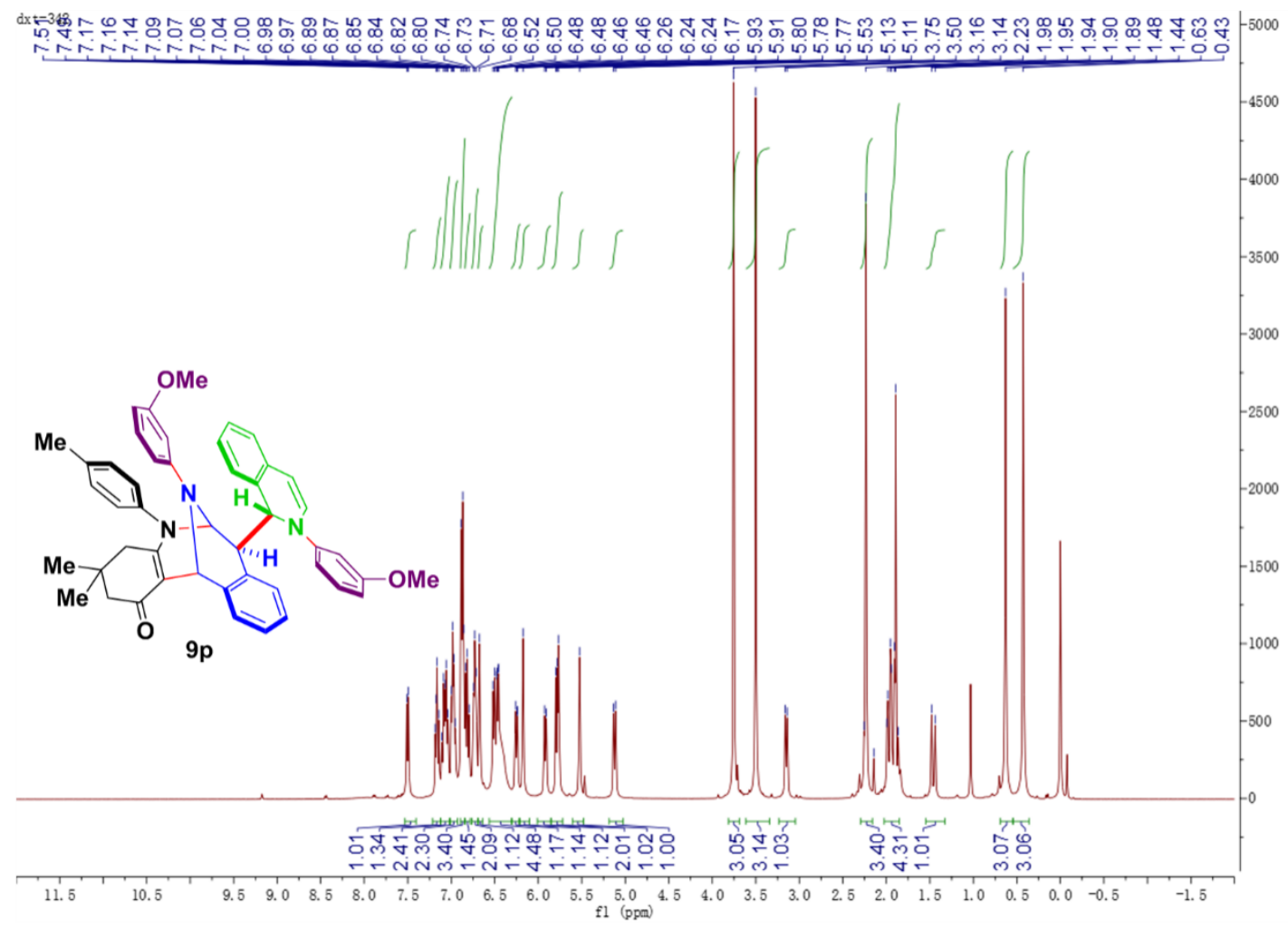

${ }^{13} \mathrm{C}$ NMR spectrum of $9 p\left(100 \mathrm{MHz}, \mathrm{CDCl}_{3}\right)$ 


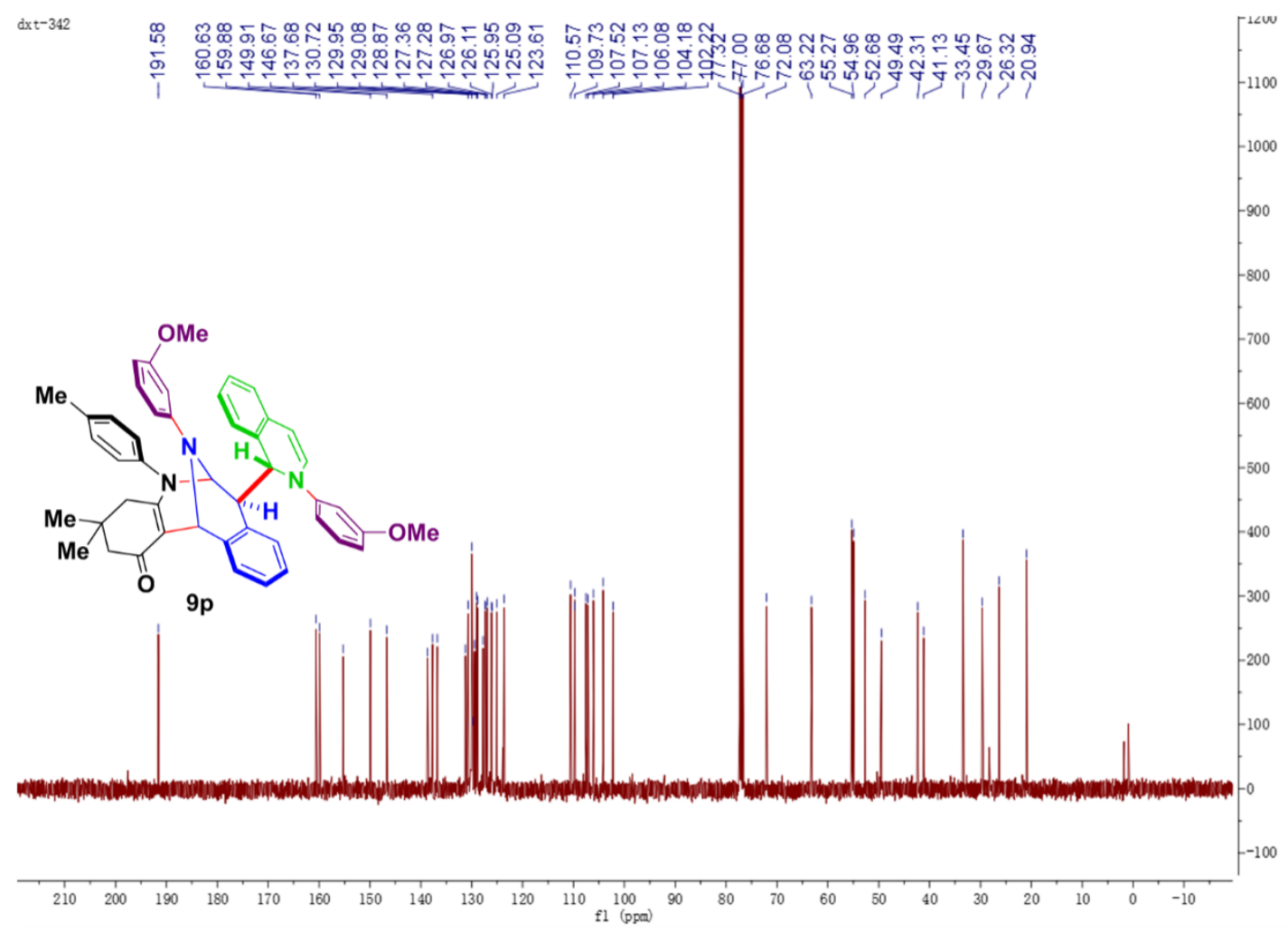

${ }^{1} \mathrm{H}$ NMR spectrum of $\mathbf{1 0}\left(400 \mathrm{MHz}, \mathrm{CDCl}_{3}\right)$

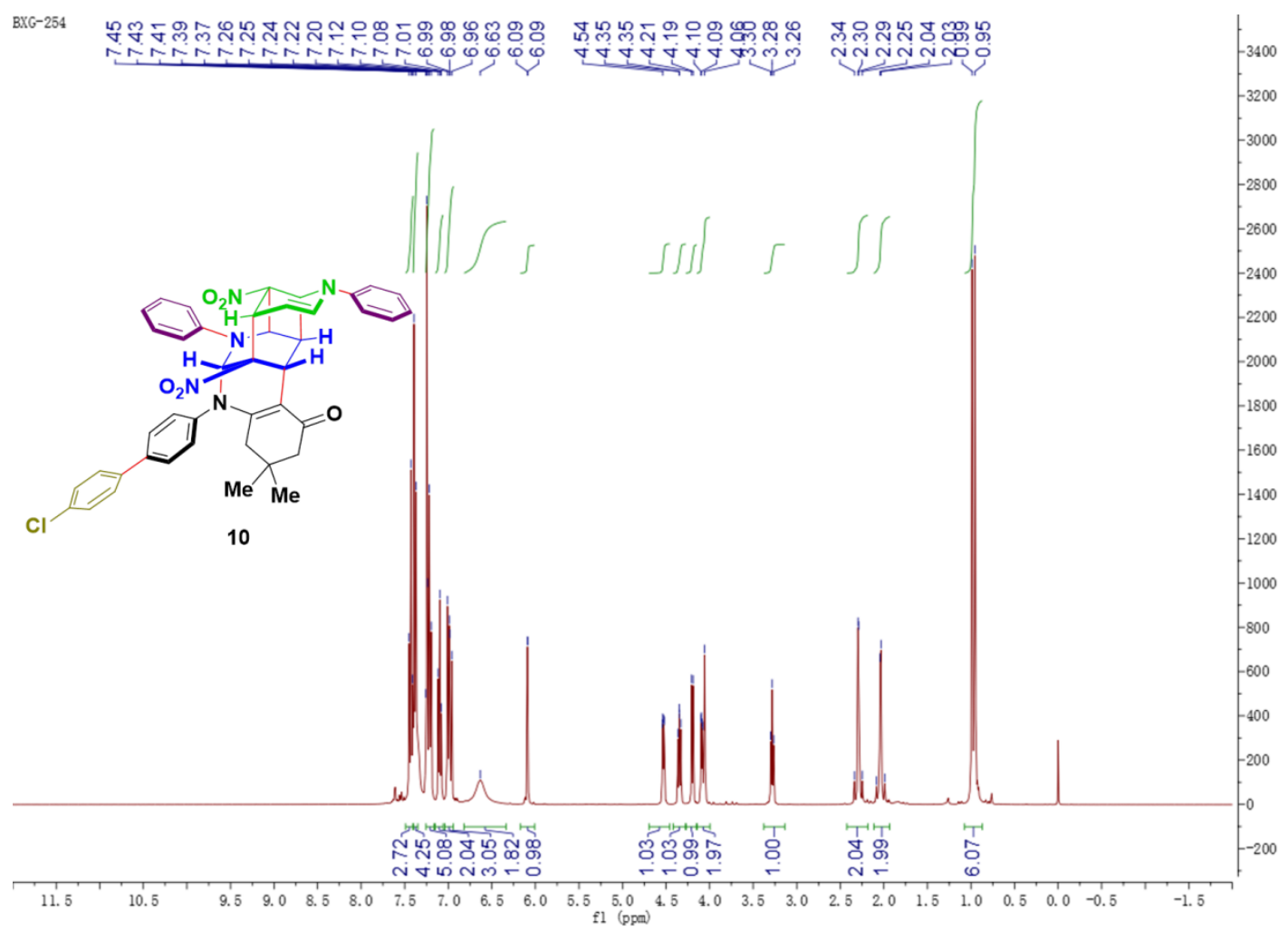

${ }^{13} \mathrm{C}$ NMR spectrum of $\mathbf{1 0}\left(100 \mathrm{MHz}, \mathrm{CDCl}_{3}\right)$ 


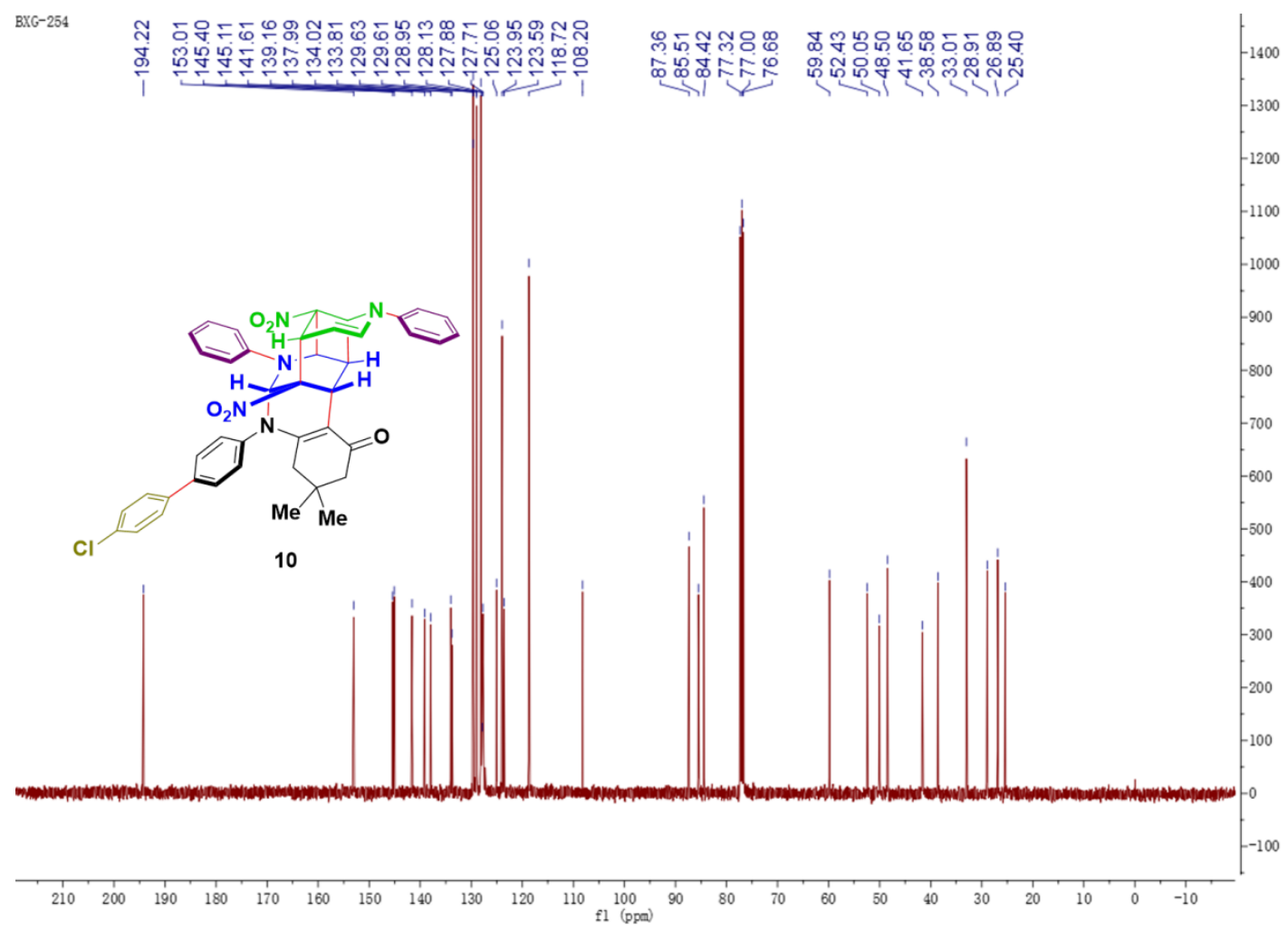

${ }^{1} \mathrm{H}$ NMR spectrum of $11\left(400 \mathrm{MHz}, \mathrm{CDCl}_{3}\right)$

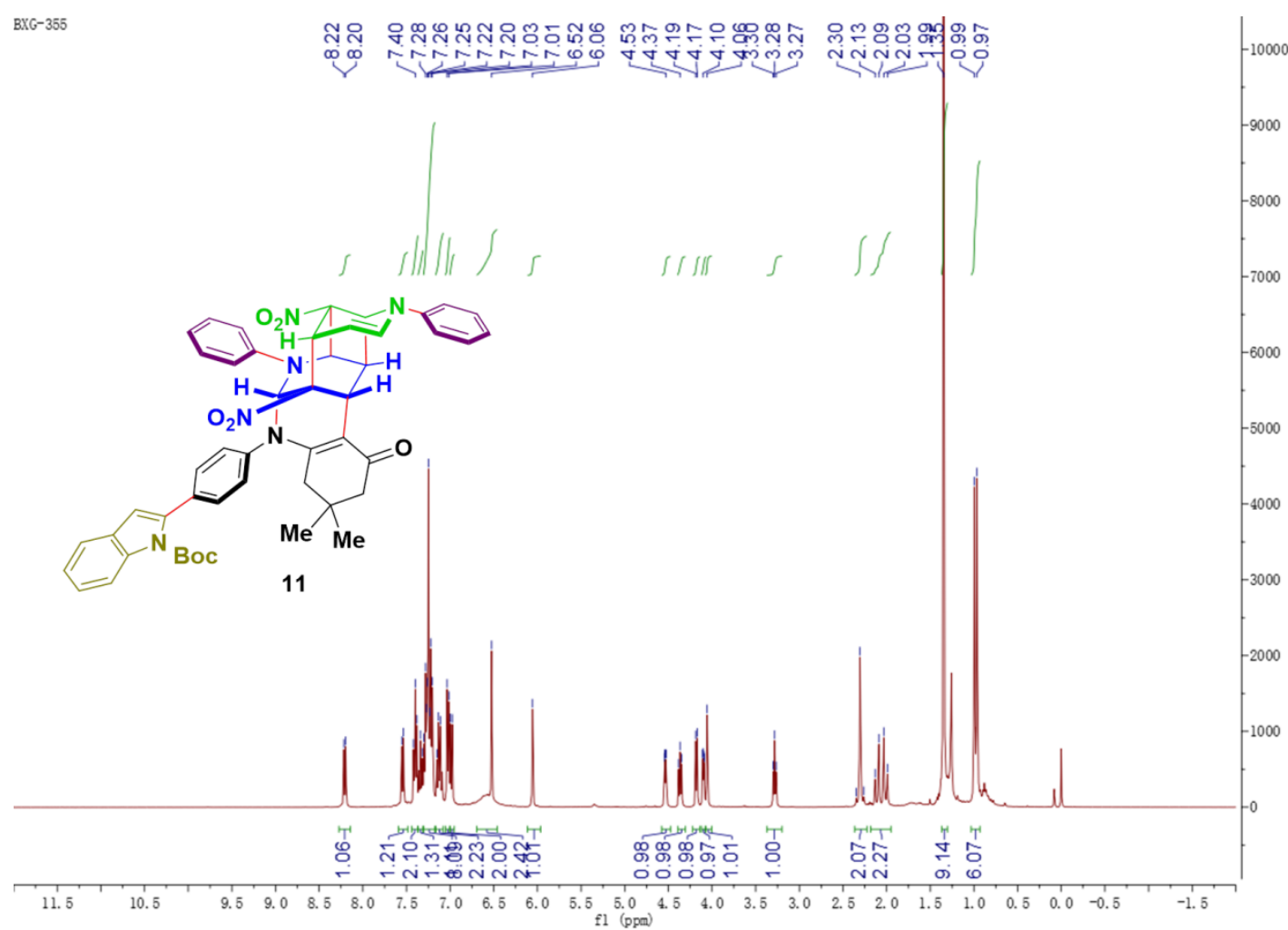

${ }^{13} \mathrm{C}$ NMR spectrum of $\mathbf{1 1}\left(100 \mathrm{MHz}, \mathrm{CDCl}_{3}\right)$ 


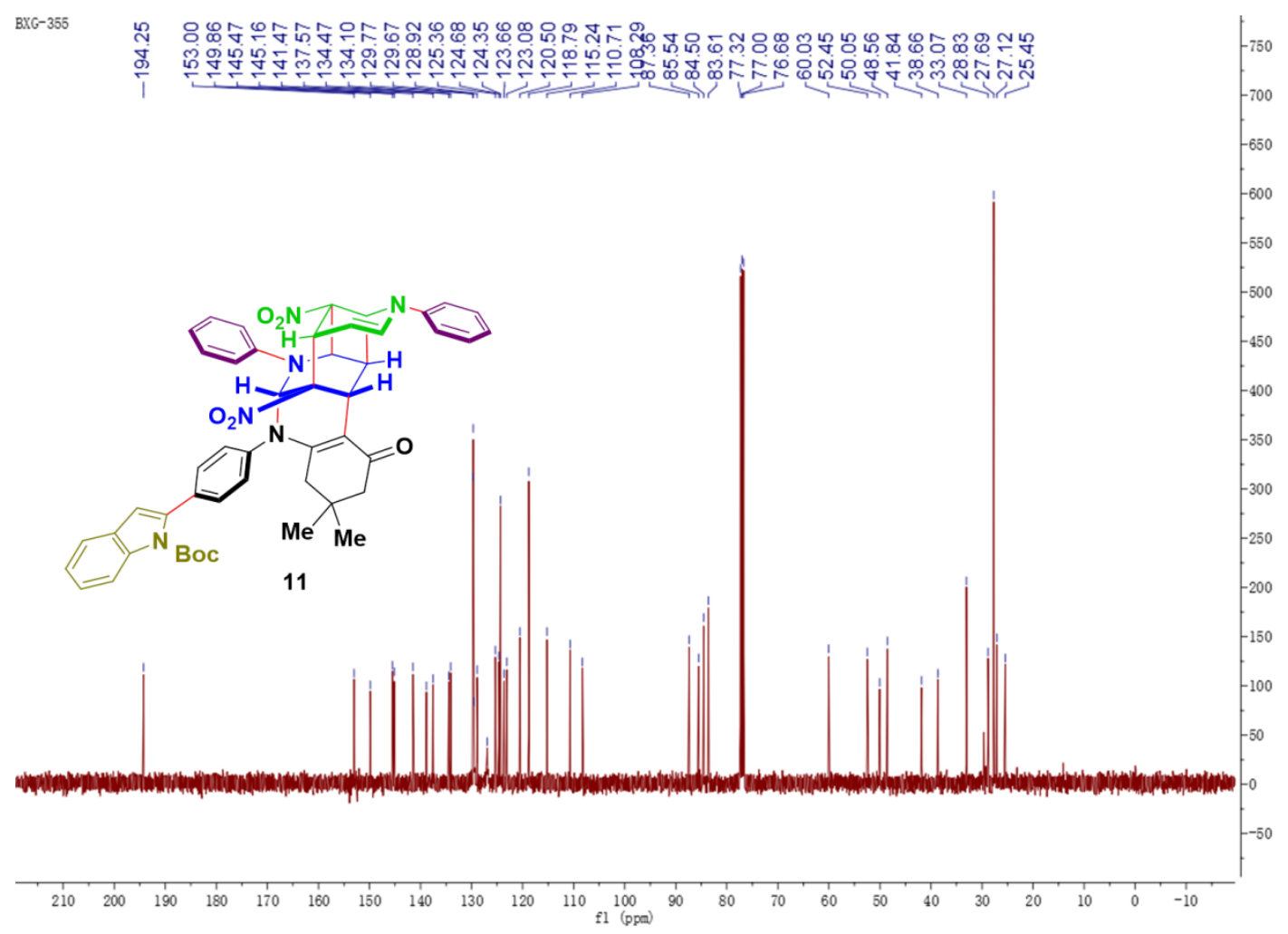

${ }^{1} \mathrm{H}$ NMR spectrum of $12\left(400 \mathrm{MHz}, \mathrm{CDCl}_{3}\right)$

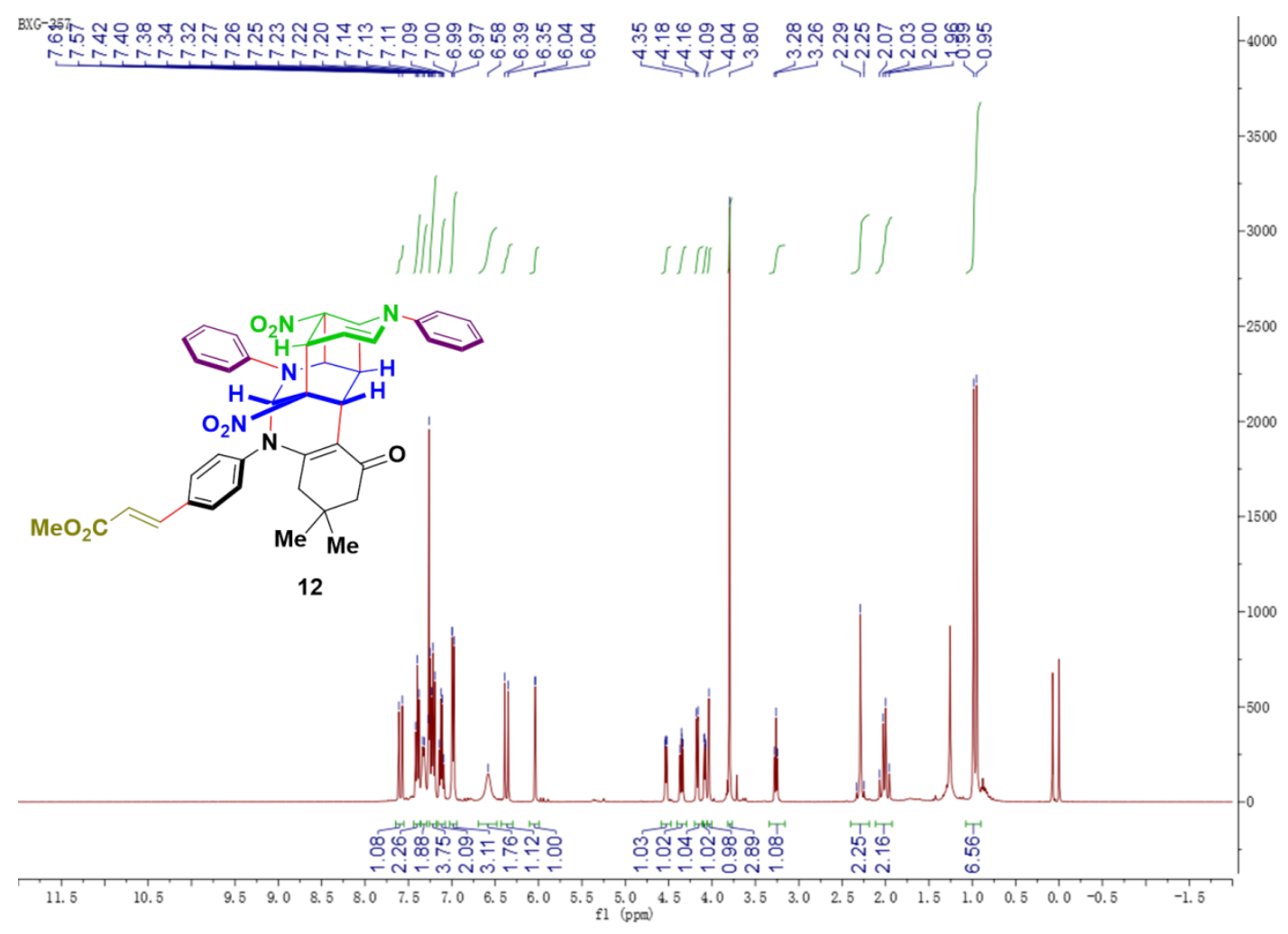

${ }^{13} \mathrm{C}$ NMR spectrum of $\mathbf{1 2}\left(100 \mathrm{MHz}, \mathrm{CDCl}_{3}\right)$ 


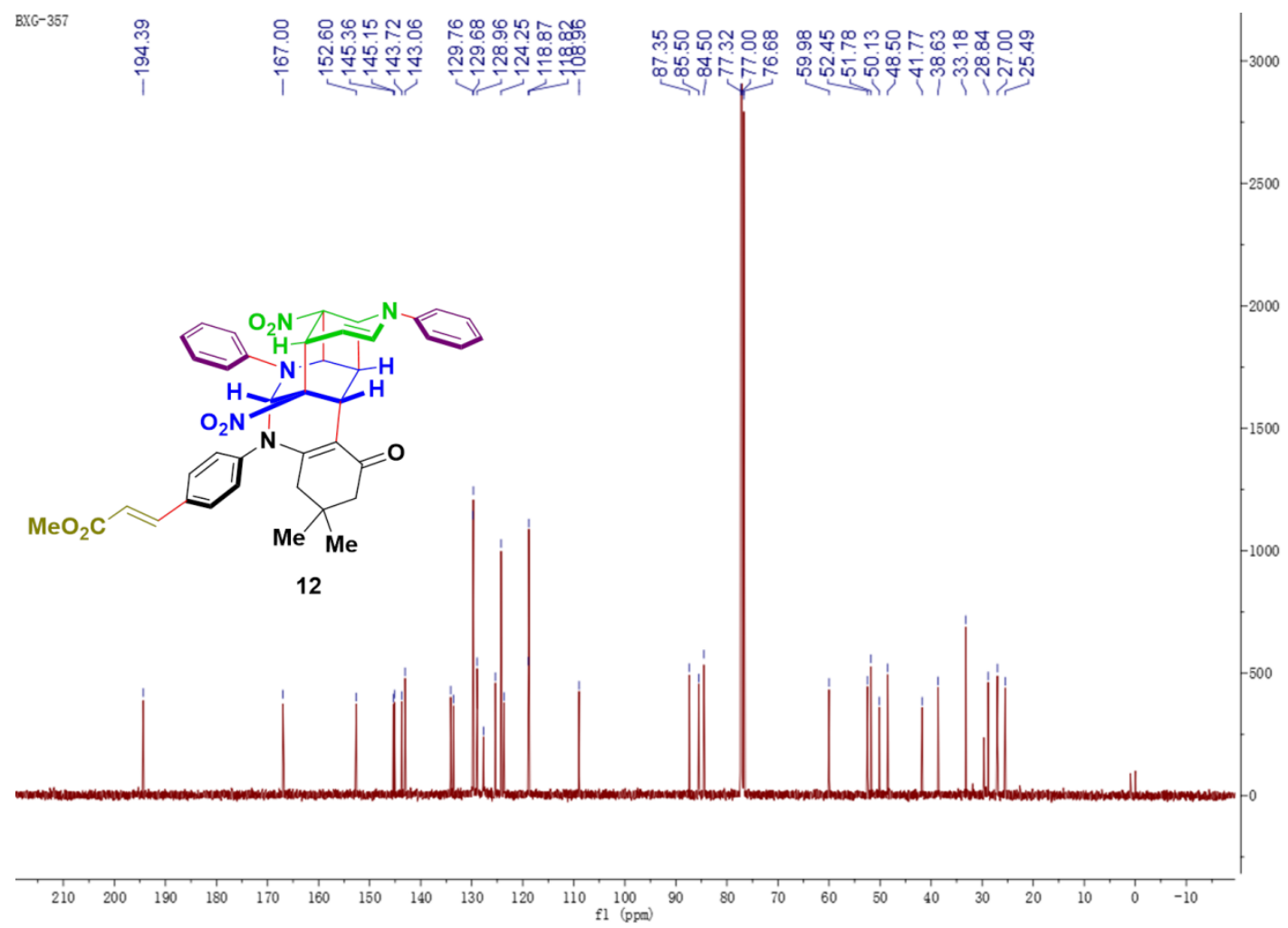

